Universidade De SÃo Paulo

Instituto DE FísicA

\title{
Modelos moleculares para fotocélulas orgânicas baseadas em hidrocarbonetos poli-aromáticos
}

\author{
Rafael Bicudo Ribeiro \\ Orientador: Márcio Teixeira do Nascimento Varella

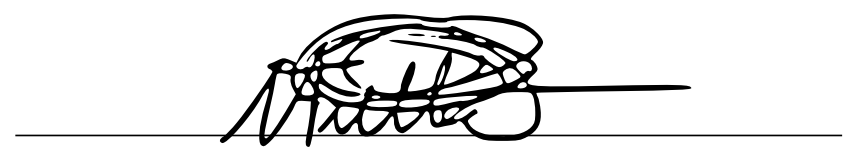 \\ Dissertação de mestrado apresentada \\ ao Instituto de Física da Universi- \\ dade de São Paulo, como requisito \\ parcial para a obtenção do título de \\ Mestre em Ciências.
}

Banca Examinadora:

Prof. Dr. Márcio Teixeira do Nascimento Varella (IFUSP)

Prof. Dr. Demétrio Antônio da Silva Filho (UnB)

Prof. Dr. Cedric Rocha Leão (UFABC) 


\section{FICHA CATALOGRÁFICA}

\section{Preparada pelo Serviço de Biblioteca e Informação}

do Instituto de Física da Universidade de São Paulo

Ribeiro, Rafael Bicudo

Modelos moleculares para fotocélulas orgânicas baseadas em hidrocarbonetos poli-aromáticos. São Paulo, 2021.

Dissertação (Mestrado) - Universidade de São Paulo. Instituto de Física. Depto. de Física Geral.

Orientador: Prof. Dr. Márcio Teixeira do Nascimento Varella

Área de Concentração: Física Atômica e Molecular

Unitermos: 1. Células solares; 2. Estrutura molecular (Física moderna); 3. Estrutura molecular (Química teórica); 4. Física teórica; 5. Física computacional. 


\title{
Molecular models for organic photocells based on polyaromatic hydrocarbons
}

\author{
Rafael Bicudo Ribeiro
}

Supervisor: Márcio Teixeira do Nascimento Varella

Master's dissertation presented to the Institute of Physics of the University of São Paulo, as a partial requirement for obtaining the title of Master of Science.

Examining Committee:

Prof. Dr. Márcio Teixeira do Nascimento Varella (IFUSP)

Prof. Dr. Demétrio Antônio da Silva Filho (UnB)

Prof. Dr. Cedric Rocha Leão (UFABC) 

Dedico aos meus avós Maria Helena e Oscar, por todo o carinho e suporte que transcendem a razão. 


\section{Agradecimentos}

Eu gostaria de começar agradecendo à Universidade de São Paulo pela oportunidade de realizar esse projeto, e ao CNPq pelo auxílio financeiro ao longo dos últimos 2 anos. O presente trabalho foi realizado com apoio do CNPq, Conselho Nacional de Desenvolvimento Científico e Tecnológico - Brasil. Agradeço aos integrantes do Instituto de Física pelo suporte e acolhimento nesses 7 anos, entre eles, equipes de limpeza, de manutenção, zeladores, técnicos de laboratório, membros da SA, da CG, CPG, da secretaria do DFGE e de tantas outras siglas, todos aqueles que contribuem silenciosamente para a nossa educação. Em especial, agradeço à Fátima, Bianca e Edineusa pela competência e simpatia, ao Cássio sempre solícito e ao Éber pela paciência e boa vontade.

Meu muito obrigado aos professores e professoras por todos os ensinamentos e pela inspiração dentro e fora da sala de aula, essenciais para o meu crescimento profissional e pessoal. Agradeço sobretudo ao Prof. Dr. Márcio Varella pela oportunidade, supervisão e orientação, que desde a graduação vêm adicionando crases e lapidando principalmente o profissional em construção dentro de mim.

Agradeço aos alunos de pós-graduação da tríade Márcio, Kaline e Sylvio, que como bons pupilos vem me auxiliando desde o funcionamento da máquina de café até o entendimento dos formalismos mais sofisticados da área de pesquisa. Notadamente, obrigado ao Julio pelos primeiros passos, ao Ely pela parceria, ao Leo Vetritti pelas conversas no metrô da linha vermelha, ao Bin pelas discussões teóricas, ao Mateus pela disponibilidade, ao Matheus especialmente pelas sublimes mensagens via cluster, ao André pelos aliases salvadores e ao Lucas, pela amizade, pelo interesse no meu trabalho e pela constante ajuda, repleta de paciência com as minhas mais diversas dúvidas. Também frutos do IF, quero agradecer aos membros do $\mathrm{T}^{*}$, amigos que foram fundamentais durante a graduação e hoje trago comigo, repleto de boas memórias. Em especial, agradeço aos três mais presentes, ao Lissinho pelo notável senso de humor, pelos extensos e expressivos diálogos e pela paciência com os meros mortais, ao Tefinho por ultrapassar o gap de aversão inicial resultando em uma bela amizade, e ao Thithi, cujas inúmeras contribuições em várias áreas compõem um conjunto infinito não enumerável. 
Agradeço à minha família, começando com a minha avó Mariazinha, por todo zelo e interesse, mesmo diante de temas complexos. Aos meus pais Marcos e Jenifer por tudo, pelo suporte nessa jornada e o amor sempre presente que transborda. Aos meus irmãos Ronivon, com suas mãos quentes e afáveis, e Frango, com sua ternura que simplesmente me conhece, meus melhores amigos e aliados da vida. Agradeço a minha companheira Beatriz, por toda sua doçura, pelo sentimento depositado e por dividir seu teto e seus sonhos comigo, me ajudando a ser alguém melhor. Por fim, agradeço a todos os meus queridos remanescentes, e, em especial, obrigado à Oxalá e toda a família espiritual que zela pela minha caminhada. 


\section{Resumo}

No contexto de células solares orgânicas, o presente trabalho investiga as propriedades do aceitador eletrônico DTP-IC-4Ph [Adv. Energy Mater. 9, 1803976 (2019)], que por meio da incorporação de moléculas $\pi$-conjugadas em estruturas do tipo FREA (Fused-Ring Electron Acceptors) resulta em dispositivos com baixo custo de produção e eficiências de conversão (PCE) promissoras. A caracterização foi realizada a nível LC-TD-DFTB (Longrange Corrected Time-Dependent Density Functional based Tight-Binding model) junto ao conjunto de parâmetros OB2 (Parametrization for Organic and Biological Molecules) com adição do átomo de enxofre. Os espectros de absorção são determinados a partir da média sobre um conjunto de coordenadas nucleares na região de Franck-Condon, que reproduz qualitativamente os resultados experimentais mas se mostra sensível a descrição do ambiente. O monômero do doador PBDB-T reafirma os resultados obtidos para o aceitador com o acréscimo de que a ausência dos demais oligômeros impacta na energia dos orbitais moleculares. Hidrocarbonetos policíclicos aromáticos (HPAs) também foram estudados, possibilitando a validação da metodologia frente ao formalismo da DFT (Teoria do Funcional de Densidade) com o funcional de correlação e troca $\omega$ B97X-D assim como a determinação de grandezas físicas como constantes dielétricas $(\epsilon)$, energias de ligação do éxciton $\left(E_{b}\right)$ e energias intramoleculares de reorganização $\left(\lambda_{e / b}\right)$. A compatibilidade entre os níveis de teoria é evidenciada comparando geometrias e espectros de absorção, e é confirmada pela baixa sensibilidade dos HPAs frente a escolha da base atômica, de tal forma que a metodologia LC-TD-DFTB se mostra uma ferramenta viável e computacionalmente favorável para a caracterização de sistemas gasosos, dependendo de correções para retratar ambientes orgânicos.

Palavras-chave: células solares orgânicas, FREA, LC-TD-DFTB, espectro de absorção 


\section{Abstract}

In the context of organic solar cells, the present work investigate properties of the electron acceptor DTP-IC-4Ph $[$ Adv. Energy Mater. 9, 1803976 (2019)], which through the addition of $\pi$-conjugated molecules in the backbone of FREA (Fused-Ring Electron Acceptors) structures results in devices with low production cost and promising conversion efficiencies (PCE). The acceptor characterization was performed at the LC-TD-DFTB (Long-range Corrected Time-Dependent Density Functional based TightBinding model) level, together with the OB2 parameter set (Parametrization of Organic and Biological Molecules) with addition of sulfur atom. Absorption spectra are determined from an average over a set of nuclear coordinates in the Franck-Condon region that qualitatively reproduces the experimental results but shows high sensitivity regarding the environment description. The PBDB-T donor monomer reaffirms the results obtained for the acceptor with the addition that the absence of other oligomers impacts the molecular orbitals energies. Polycyclic aromatic hydrocarbons (PAHs) were also studied, enabling the methodology validation against DFT (Density Functional Theory) formalism with the $\omega$ B97X-D correlation and exchange functional as well as the determination of physical quantities as dielectrics constants $(\epsilon)$, exciton binding energies $\left(E_{b}\right)$ and intramolecular reorganization energies $\left(\lambda_{e / b}\right)$. The compatibility between both levels of theory is evidenced by comparing geometries and absorption spectra, being confirmed by the low sensitivity of HPAs towards the choice of atomic basis, in such a way that the LC-TD-DFTB methodology proves to be a feasible and computationally favorable tool for the characterization of gas phase systems, depending on corrections to properly portray organic environments.

Keywords: organic solar cells, FREA, LC-TD-DFTB, absorption spectrum 


\section{Lista de Símbolos}

$\alpha$
$\beta$
$\delta$
$\epsilon$
$\theta$
$\lambda$
$\mu, \boldsymbol{\mu}$
$\rho$
$\sigma$
$\tau$
$\Psi$
$\psi, \varphi, \phi$
$\chi$
$\Omega$
$\omega$
$\nabla$
$\nabla^{2}$
$\mathcal{A}$
$E A$
$E, E_{b}, \mathcal{E}$
$E_{x c}$
$E_{x}$
$E_{c}$
$e$
$F[\rho]$
$f$
$g$
$\hat{\mathcal{H}}, \hat{H}$
$\hbar$
$I P$
$J$
$K$
$k$
$m_{e}$
$n$

Projeção de spin positiva, resposta linear, polarizabilidade

Projeção de spin negativa

Delta de Dirac, delta de Kronecker

Autovalor de energia, constante dielétrica

Função de Heaviside

Energia intramolecular de reorganização

Potencial químico, momento de dipolo

Densidade eletrônica, densidade de estados

Projeção de spin, densidade de carga superficial, seção de choque

Constante de decaimento atômica

Função de onda global

Spin-orbitais e orbitais atômicos

Autofunção nuclear, função de resposta retardada

Energia de excitação

Parâmetro de longo alcance, frequência angular

Gradiente

Laplaciano

Operador de antissimetrização

Afinidade eletrônica

Energia, energia de ligação do éxciton,

superfície de energia potencial, campo elétrico

Energia de correlação e troca

Energia de troca

Energia de correlação eletrônica

Carga do elétron

Funcional universal

Kernel, número de ocupação, função de atenuação, função de pontuação, força de oscilador

Função gaussiana normalizada

Operador hamiltoniana

Constante de Planck reduzida

Potencial de ionização

Potencial de Coulomb, critério de otimização

Matriz de acoplamento

mobilidade dos portadores de carga

Massa do elétron

Número de ocupação, índice de refração 


$\begin{array}{ll}\boldsymbol{P}, P & \text { Matriz de densidade, vetor de polarização, distribuição de probabilidade } \\ p & \text { Polarizabilidade atômica } \\ q & \text { Carga de transição atômica, carga de Mulliken } \\ R(r) & \text { Solução radial do átomo de hidrogênio } \\ \boldsymbol{r}, \boldsymbol{R}, \hat{\mathcal{R}} & \text { Vetor ou operador de posição } \\ S & \text { Matriz de overlap, estado excitado } \\ \hat{T} & \text { Operador de energia cinética } \\ U & \text { Parâmetro de Hubbard } \\ \hat{U} & \text { Operador de evolução temporal } \\ \hat{V}, \hat{W}, v, w & \text { Operador de interação } \\ V & \text { Potencial eletrostático, volume } \\ v_{x c} & \text { Potencial de correlação e troca } \\ Y(\theta, \phi) & \text { Harmônico esférico } \\ Z & \text { Número atômico }\end{array}$




\section{Lista de Figuras}

1.1 Adaptação do infográfico fornecido pela NREL [6] com a evolução da eficiência de diferentes células solares. . . . . .

1.2 Representação esquemática das camadas de uma célula solar e do interior da camada ativa. Adaptado das referências [7, 8]. 22

1.3 Seção transversal da camada ativa e as etapas da dinâmica interna das OSC. (1) Absorção de radiação e criação dos éxcitons; (2) difusão dos éxcitons até a interface D:A; (3) dissociação dos éxcitons; (4) movimentação dos portadores de carga em direção aos eletrodos; (5) extração dos portadores de carga. Adaptado da referência [9]. . . . . . . . . .

1.4 Estrutura química de exemplares NFAs com diferentes gaps de energia. Adaptado da referência [9]. . . . . . . . . . . .

1.5 Representação de uma estrutura FREA. A figura foi adaptada da referência [15]. . . . . . . . . . . . . . . .

1.6 Figura com as representações em linha dos compostos desenvolvidos por Wang et al. [15]. . . . . . . . . . . . . .

1.7 Representação do PBDB-T e de outros doadores derivados. Adaptação da referência [27]. . . . . . . . . . . . . . . . . .

2.1 Escada de aproximações, adaptada da referência [34], que parte da teoria de Hartree e ilustra o rumo em direção ao ganho de precisão introduzindo as diferentes classes de funcionais. Cada degrau inclui novos elementos na formulação dos mesmos. . . . . . . . . . . . . . . . . .

3.1 Isosuperfícies dos orbitais HOMO (primeira linha) e LUMO (segunda linha) da molécula de pireno para diferentes bases atômicas. O módulo do isovalor é fixado em 0.3 . . . . . . . .

3.2 Espectros de absorção da molécula de naftaleno, obtidos variando a base atômica na geometria otimizada com a base 6-31G(d,p). No painel de cima são apresentados os resultados para as funções de Pople e no debaixo para as funções da família cc (correlated-consistent). Foram considerados 50 estados excitados em cada cálculo. . . . . . . . . . . . 
3.3 Espectros de absorção da molécula de pireno, obtidos variando a base atômica na geometria otimizada com a base 6-31G(d,p). No painel de cima são apresentados os resultados para as funções de Pople e no debaixo para as funções da família cc (correlated-consistent). Foram considerados 50 estados excitados em cada cálculo. . . . . . . . . . . .

3.4 Representação dos hidrocarbonetos poliaromáticos estudados no projeto.

3.5 Critérios de otimização $J_{1}$ e $J_{3}$ em função do parâmetro de longo alcance $(\omega)$ para a molécula de naftaleno. No painel superior os valores foram obtidos utilizando o potencial de ionização $(I P)$ e a afinidade eletrônica $(E A)$ calculados, enquanto no painel inferior foram utilizados os valores experimentais.

3.6 Critérios de otimização $J_{1}$ e $J_{3}$ em função do parâmetro de longo alcance $(\omega)$ para a molécula de pireno. No painel superior os valores foram obtidos utilizando o potencial de ionização $(I P)$ e a afinidade eletrônica $(E A)$ calculados, enquanto no painel inferior foram utilizados os valores experimentais.

3.7 Comportamento das contribuições de curto e longo alcance do termo de Coulomb. Nas legendas o número entre parênteses corresponde ao valor de $\omega$. (a) Funções $\frac{\operatorname{erf}\left(\omega r_{12}\right)}{r_{12}}$ e $\frac{\operatorname{erfc}\left(\omega r_{12}\right)}{r_{12}}$ com $\omega=0.1$ e 0.5. (b) Funções $\frac{e^{-\omega r_{12}}}{r_{12}}$ e $\frac{1-e^{-\omega r_{12}}}{r_{12}} \operatorname{com} \omega=0.1$ e 0.5 . (c) Comparação entre as decomposições para um mesmo valor de $\omega$. (d) Funções de atenuação das energias de troca $E_{x}^{\mathrm{SR}-\mathrm{B} 97}(\mathrm{C} .2)$ e $E_{x}^{\omega, L D A}(2.70) . \ldots \ldots \ldots$

3.8 Critérios de otimização $J_{1}$ e $J_{3}$ em função do parâmetro de longo alcance $(\omega)$ para o composto DTP-IC-4Ph com e sem cadeias laterais. $\mathrm{O}$ símbolo ${ }^{\dagger}$ identifica o composto sem cadeias laterais.

3.9 Espectros de absorção da molécula de naftaleno obtidos utilizando ambas os métodos. Os cálculos de estados excitados foram realizados na geometria otimizada via $\mathrm{DFT} / \omega \mathrm{B} 97 \mathrm{X}-$ D/6-31G(d,p). Foram considerados 100 estados excitados em cada cálculo. . . . . . . . . . . . . . . . . . . 
3.10 Espectros de absorção da molécula de naftaleno obtidos utilizando ambas as metodologias. A legenda faz referência aos métodos DFT e DFTB, utilizados tanto na otimização de geometria quanto no cálculo de estados. Foram considerados 100 estados excitados em cada cálculo. . . . . . . . . . .

3.11 Espectros de absorção da molécula de antraceno obtidos utilizando ambas as metodologias. A legenda faz referência aos métodos DFT e DFTB, utilizados tanto na otimização de geometria quanto no cálculo de estados. Foram considerados 100 estados excitados em cada cálculo. . . . . . . . . . .

3.12 Espectros de absorção da molécula de pireno obtidos utilizando ambas as metodologias. A legenda faz referência aos métodos DFT e DFTB, utilizados tanto na otimização de geometria quanto no cálculo de estados. Foram considerados 100 estados excitados em cada cálculo. . . . . . . . . . .

3.13 Espectros de absorção do composto DTP-IC-4Ph. A curva azul descreve o composto como apresentado na figura, enquanto a curva verde troca as cadeias laterais $\mathrm{C}_{6} \mathrm{H}_{13}$ por átomos de hidrogênio. . . . . . . . . . . . . . .

3.14 Isosuperfícies dos orbitais HOMO (painel superior) e LUMO (painel inferior) do aceitador DTP-IC-4Ph com (a) e sem (b) cadeias laterais, calculados via DFTB com $\omega=0.1 a_{0}^{-1}$. O módulo do isovalor é fixado em 0.015 . . . . . . . . . . .

3.15 Espectros de absorção normalizados do composto DTP-IC$4 \mathrm{Ph}$. As curvas tracejadas dizem respeito aos dados experimentais, obtidos por Wang et al. [15] a partir de um filme fino e de uma solução com clorofórmio $\left(\mathrm{CHCl}_{3}\right)$ como solvente, enquanto a curva sólida corresponde ao espectro, na ausência de cadeias laterais (identificadas como $\mathrm{C}_{6} \mathrm{H}_{13}$ na imagem do composto), determinado a partir de 1000 configurações via DFTB. . . . . . . . . . . . . . . . . .

3.16 Espectros de absorção do composto DTP-IC-4Ph. No painel superior são apresentadas as intensidades absolutas e no inferior as intensidades normalizadas. As curvas verde e azul correspondem aos espectros verticais do aceitador com e sem cadeias laterais, respectivamente. A curva laranja corresponde ao espectro, na ausência de cadeias laterais, determinado a partir de 1000 configurações via $D F T B$. Todos os cálculos adotam o valor otimizado $\omega=0.1 a_{0}^{-1} \ldots$. . . . 
3.17 Espectros de absorção do composto PBDB-T. A curva azul descreve o monômero do composto $(n=1)$, enquanto as demais curvas correspondem ao espectro na ausência de cadeias laterais. A linha verde utiliza o valor de $\omega$ otimizado na ausência de cadeias laterais ( $\omega=0.2 a_{0}^{-1}$ ), enquanto a pontilhada utiliza o valor otimizado para o composto na íntegra $\left(\omega=0.1 a_{0}^{-1}\right) \ldots \ldots \ldots \ldots \ldots \ldots \ldots$

3.18 Isosuperfícies dos orbitais HOMO (primeira linha) e LUMO (segunda linha) do doador PBDB-T com e sem cadeias laterais, calculados via DFTB. As duas primeiras colunas utilizaram $\omega=0.1 a_{0}^{-1}$ e a terceira $\omega=0.2 a_{0}^{-1}$. O módulo do isovalor é fixado em 0.015 .

3.19 Espectros de absorção normalizados do PBDB-T. A linha tracejada diz respeito aos dados experimentais, fornecidos por Sunsun et al. [24], enquanto as linhas sólida correspondem aos espectros do monômero, determinados a partir de 1000 configurações via DFTB. A curva verde corresponde ao espectro do composto como representado na figura e a amarela na ausência das cadeias laterais.

3.20 Orbitais HOMO (à esquerda) e LUMO (à direita) da molécula de pireno otimizada a nível $\omega$ B97X-D/6-31G(d,p)/PCM com clorofórmio como solvente.

3.21 Espectros de absorção dos compostos HPAs à vácuo e solvatados com água $\left(\mathrm{H}_{2} \mathrm{O}\right)$ e clorofórmio $\left(\mathrm{CHCl}_{3}\right)$. Os cálculos foram realizados através do software Gaussian09 [88] em nível TD-DFT/ $/$ B97X-D/6-31G(d,p) com PCM, envolvendo 100 estados excitados.

O.1 Superfície delimitada pelas esferas de van-der-Waals cujo interior estima o volume do composto, nesse caso, o aceitador DTP-IC-4Ph sem as cadeias laterais.

O.2 Evolução do volume para a molécula de tiofeno. O painel superior apresenta o volume $(V)$ em função do número de pontos $(N)$ para os primeiros 2500 pontos. O histograma inferior contabiliza as contribuições desses primeiros 2500 pontos. 


\section{Lista de Tabelas}

3.1 Raízes do desvio quadrático médio (RMSD) dos comprimentos de ligação em relação a geometria otimizada com a base atômica $6-31 \mathrm{G}(\mathrm{d}, \mathrm{p})$ para as moléculas de naftaleno e pireno. As duas últimas linhas dizem respeito a média e o desvio absoluto médio.

3.2 Energias do estado fundamental, dos orbitais HOMO $\left(\epsilon_{\text {Номо }}\right)$ e LUMO $\left(\epsilon_{L U M O}\right)$ assim como suas diferenças $E_{\text {gap }}^{H-L}$, calculadas para a molécula de naftaleno utilizando o funcional $\omega \mathrm{B} 97 \mathrm{X}-\mathrm{D}$ e variando a base atômica. As duas últimas linhas dizem respeito a média e o desvio absoluto médio desconsiderando a base STO-3G. . . . . . . . . . . . . . . .

3.3 Energias do estado fundamental, dos orbitais HOMO $\left(\epsilon_{\text {HOMO }}\right)$ e LUMO $\left(\epsilon_{L U M O}\right)$ assim como suas diferenças $E_{\text {gap }}^{H-L}$, calculadas para a molécula de pireno utilizando o funcional $\omega \mathrm{B} 97 \mathrm{X}-$ $\mathrm{D}$ e variando a base atômica. As duas últimas linhas dizem respeito a média e o desvio absoluto médio desconsiderando a base STO-3G. . . . . . . . . . . . . . . .

3.4 Energias de transição, e respectivas forças de oscilador $(f)$, do primeiro estado excitado e do primeiro estado brilhante da molécula de naftaleno. Na geometria otimizada via $\omega$ B97X$\mathrm{D} / 6-31 \mathrm{G}(\mathrm{d}, \mathrm{p})$ os estados excitados foram calculados variando a base atômica. As duas últimas linhas dizem respeito a média e o desvio absoluto médio desconsiderando a STO-3G. 82

3.5 Energias de transição, e respectivas forças de oscilador $(f)$, do primeiro estado excitado e do primeiro estado brilhante da molécula de pireno. Na geometria otimizada via $\omega$ B97X$\mathrm{D} / 6-31 \mathrm{G}(\mathrm{d}, \mathrm{p})$ os estados excitados foram calculados variando a base atômica. As duas últimas linhas dizem respeito a média e o desvio absoluto médio desconsiderando a STO-3G. 83

3.6 Critérios de otimização $J_{1}$ e $J_{3}$ calculados para a o molécula de naftaleno variando o parâmetro de longo alcance $(\omega)$ no intervalo de 0.1 até $0.5 a_{0}^{-1} \ldots \ldots \ldots \ldots$. . . . . . . 
3.7 Parâmetros de longo alcance otimizados e respectivos valores de $J_{1}$ e $J_{3}$ para os compostos de naftaleno, antraceno e pireno, usando ambas as metodologias. . . . . . . . . .

3.8 Potenciais de ionização (IP) das moléculas de naftaleno, antraceno e pireno obtidos via DFTB. As linhas com $\omega=$ $0.5 a_{0}^{-1}$ dizem respeito ao valor otimizado a partir das energias verticais enquanto $\omega=0.3 a_{0}^{-1}$ utiliza dos dados experimentais. Os valores entre parênteses na coluna IP $\mathrm{EXP}_{\text {dizem }}$ respeito as incertezas experimentais. . . . . . . . . . . .

3.9 Critérios de otimização $J_{1}$ e $J_{3}$ para o composto DTP-IC$4 \mathrm{Ph}$ variando o parâmetro de longo alcance $(\omega)$ no intervalo de 0.1 até $0.5 a_{0}^{-1}$. A coluna DTP-IC-4Ph ${ }^{\dagger}$ diz respeito aos valores obtidos na ausência de cadeias laterais. . . . . . . .

3.10 Energias dos orbitais HOMO $\left(\epsilon_{H O M O}\right)$ e LUMO $\left(\epsilon_{L U M O}\right)$ assim como suas diferenças $E_{\text {gap }}^{H-L}$ calculadas para o composto DTP-IC-4Ph variando o parâmetro de longo alcance $(\omega)$ no intervalo de 0.1 até $0.5 a_{0}^{-1}$. A coluna DTP-IC-4Ph ${ }^{\dagger}$ diz respeito aos valores obtidos na ausência de cadeias laterais.

3.11 Critérios de otimização $J_{1}$ e $J_{3}$ para o composto PBDB-T variando o parâmetro de longo alcance $(\omega)$ no intervalo de 0.1 até $0.5 a_{0}^{-1}$. A coluna $\mathrm{PBDB}^{-} \mathrm{T}^{\dagger}$ diz respeito aos valores obtidos na ausência de cadeias laterais. . . . . . . . . . .

3.12 Energias dos orbitais HOMO $\left(\epsilon_{\text {HOMO }}\right)$ e LUMO $\left(\epsilon_{L U M O}\right)$ assim como suas diferenças $E_{\text {gap }}^{H-L}$ calculadas para o composto PBDB-T variando o parâmetro de longo alcance $(\omega)$ no intervalo de 0.1 até $0.5 a_{0}^{-1}$. A coluna $\mathrm{PBDB}-\mathrm{T}^{\dagger}$ diz respeito aos valores obtidos na ausência de cadeias laterais. . . . . .

3.13 Gap de energia obtido experimentalmente e através dos métodos DFT e DFTB adotando o parâmetro de longo alcance otimizado. Na coluna DFTB são apresentados os valores otimizados teórico e experimental, respectivamente. Os valores entre parênteses na coluna Exp dizem respeito as incertezas experimentais. . . . . . . . . . . . . .

3.14 Raízes do desvio quadrático médio (RMSD) dos comprimentos de ligação em relação a geometria otimizada via $D F T$ sem PCM, para as moléculas de naftaleno e pireno. . . . .

3.15 Energias dos orbitais HOMO $\left(\epsilon_{\text {HOMO }}\right)$ e as diferenças entre HOMO e LUMO, $E_{\text {gap }}^{H-L}$, calculadas para os HPAs solvatados em clorofórmio e água a nível PCM. . . . . . . . . . . . . . 
3.16 Volume $(V)$, polarizabilidade isotrópica $\left(\alpha_{0}\right)$ e módulo do momento de dipolo permanente $(\mu)$ dos compostos naftaleno, antraceno, pireno, DTP-IC-4Ph e do monômero de PBDB$\mathrm{T}$, calculados com ambos os métodos. As linhas PBDB- $\mathrm{T}^{\dagger} \mathrm{e}$ $\mathrm{DTP}-\mathrm{IC}-4 \mathrm{Ph}^{\dagger}$ dizem respeito aos valores obtidos na ausência de cadeias laterais. . . . . . . . . . . . . . . .

3.17 Constantes dielétricas $(\epsilon)$ dos compostos naftaleno, antraceno, pireno, DTP-IC-4Ph e do monômero de PBDB-T, obtidos com ambos os métodos e experimentalmente. As linhas PBDB- $\mathrm{T}^{\dagger}$ e DTP-IC-4Ph ${ }^{\dagger}$ dizem respeito aos valores obtidos na ausência de cadeias laterais. . . . . . . . . . . . .

3.18 Constantes dielétricas $(\epsilon)$ dos compostos naftaleno, antraceno, pireno, DTP-IC-4Ph e do monômero de PBDB-T, obtidas experimentalmente e via ajuste. As linhas $\mathrm{PBDB}^{-\mathrm{T}^{\dagger}} \mathrm{e}$ DTP-IC-4Ph ${ }^{\dagger}$ dizem respeito aos valores obtidos na ausência de cadeias laterais.

3.19 Energias de ligação do éxciton $\left(E_{b}\right)$ dos compostos naftaleno, antraceno e pireno, DTP-IC-4Ph e monômero de PBDB-T calculadas utilizando ambas os métodos. As linhas PBDB$\mathrm{T}^{\dagger}$ e DTP-IC-4Ph dizem respeito aos valores obtidos na ausência de cadeias laterais. . . . . . . . . . . . . .

3.20 Energias intramoleculares de reorganização internas dos buracos e elétrons $\left(\lambda_{i n}^{b / e}\right)$ dos compostos naftaleno, antraceno e pireno calculadas utilizando ambas as metodologias. . . . .

C.1 Contribuições para a energia total de correlação dinâmica.

C.2 Parâmetros otimizados para o funcional $\omega$ B97X-D. . . . . .

K.1 Parâmetros considerados na correção de dispersão do modelo de Slater-Kirkwood. As colunas com polarizabilidades atômicas e raios de van der Waals fornecem os valores variando o número de primeiros vizinhos de 0 até 5 ou mais, respectivamente. . . . . . . . . . . .

L.1 Raízes do desvio quadrático médio (RMSD) dos comprimentos de ligação, obtidos via $D F T B$, em relação a geometria otimizada a nível $\omega$ B97X-D/6-31G(d,p), para as moléculas de naftaleno, antraceno e pireno. As duas últimas linhas dizem respeito a média e o desvio absoluto médio. . . . . . 
L.2 Raízes do desvio quadrático médio (RMSD) dos comprimentos de ligação, obtidos via $D F T B$, em relação a geometria otimizada com $\omega=0.3 a_{0}^{-1}$, para as moléculas de naftaleno, antraceno e pireno. As duas últimas linhas dizem respeito a média e o desvio absoluto médio. . . . . . . . . . . . .

L.3 Raízes do desvio quadrático médio (RMSD) dos comprimentos de ligação, obtidos via $D F T B$, em relação a geometria otimizada com $\omega=0.5 \mathrm{a}_{0}^{-1}$, para as moléculas de naftaleno, antraceno e pireno. As duas últimas linhas dizem respeito a média e o desvio absoluto médio. . . . . . . . . . . . .

M.1 Energias de transição e respectivas forças de oscilador $(f)$ dos primeiros 5 estados excitados $\left(S_{i}\right)$ e dos primeiros 5 estados oticamente ativos $\left(S_{B i}\right)$ dos compostos naftaleno, antraceno e pireno. Os resultados foram obtidos com os métodos DFT e DFTB, utilizando o parâmetro de longo alcance otimizado via $J_{3}\left(\omega=0.2 a_{0}^{-1}\right.$ para $D F T$ e $\omega=0.5 a_{0}^{-1}$ para $D F T B)$ e via $J_{3}^{\text {exp }}\left(\omega=0.3 a_{0}^{-1}\right.$ para DFTB $) \ldots \ldots .$.

O.1 Volumes das moléculas de água, amônia, metano, etanol, benzeno e tiofeno, obtidos utilizando ambas as metodologias. A coluna DP diz respeito ao desvio padrão associado à média de 10 cálculos com $10^{6}$ pontos em cada um. . . . . . . . 182 


\section{Lista de Abreviaturas e Siglas}

$\begin{array}{ll}\text { ASC } & \text { Apparent Surface Charge } \\ \text { BHJ } & \text { Bulk Heterojunction } \\ \text { DFT } & \text { Density Functional Theory } \\ \text { DFTB } & \text { Density Functional based Tight-Binding } \\ \text { DSSC } & \text { Dye-Sensitized Solar Cells } \\ \text { EA } & \text { Electron Affinity } \\ \text { EPBT } & \text { Energy Pay Back Time } \\ \text { FREA } & \text { Fused-Rings Electron Acceptors } \\ \text { GB } & \text { Generalized Born method } \\ \text { HF } & \text { Hartree-Fock } \\ \text { HK } & \text { Hohenberg-Kohn } \\ \text { HOMO } & \text { Highest Ocuppied Molecular Orbital } \\ \text { HPA } & \text { Hidrocarbonetos poli-aromáticos } \\ \text { IP } & \text { Ionization Potential } \\ \text { KS } & \text { Kohn-Sham } \\ \text { LC } & \text { Long-range Corrected } \\ \text { LC-TD-DFTB } & \text { Long-range Corrected Time-Dependent Density Functional } \\ \text { LCA } & \text { based Tight-Binding method } \\ \text { LDA } & \text { Life Cycle Analysis } \\ \text { LR-TDDFT } & \text { Local Density Approximation } \\ \text { LSDA } & \text { Linear Response Time-Dependent Density Functional Theory } \\ \text { MP2 } & \text { Local Spin-Density Approximation } \\ \text { NEA } & \text { Teoria de perturbação Møller-Plesset de 2 ordem } \\ \text { NFAs } & \text { Nuclear Ensemble Approach } \\ \text { NREL } & \text { Non-Fullerene Electron Acceptors } \\ \text { OSC } & \text { National Renewable Energy Laboratory } \\ \text { PCE } & \text { Organic Solar Cells } \\ \text { PCM } & \text { Power Conversion Energy } \\ \text { RMSD } & \text { Polarizable Continuum Model } \\ \text { SCC-DFTB } & \text { Root-Mean-Square Deviation } \\ \text { SCLC } & \text { Self-Consistent Charge Corrected Density Functional } \\ \text { TDDFT } & \text { Sased Tight-Binding } \\ \text { TDDFTB } & \text { Time-Dependent Density Functional Theory } \\ \text { xc } & \text { Time-Depedent Density Functional based Tight-Binding } \\ & \end{array}$




\section{Sumário}

1 Introdução $\quad 20$

1.1 Células solares emergentes . . . . . . . . . . . . . 20

1.2 As estruturas FREAs . . . . . . . . . . . . . . . 23

$1.3 \mathrm{O}$ aceitador DTP-IC-4Ph . . . . . . . . . . 26

1.4 A dissertação e seus objetivos . . . . . . . . . . . . . . 28

2 Metodologias 31

2.1 Aproximação Born-Oppenheimer . . . . . . . . . . . . . 31

2.2 Teoria do Funcional de Densidade . . . . . . . . . . . . . 34

2.2.1 Teorema de Hohenberg-Kohn . . . . . . . . . . . 36

2.2.2 Equações de Kohn-Sham . . . . . . . . . . . . . . . . 39

2.2.3 O funcional de correlação e troca $\omega$ B97X-D . . . . . . 41

2.2.4 A base atômica 6-31G(d,p) . . . . . . . . . . 44

2.3 Teoria do Funcional de Densidade Dependente do Tempo . . 46

2.3.1 Teorema de Runge-Gross . . . . . . . . . . . . . 46

2.3.2 Teorema de van Leeuwen . . . . . . . . . . . . . . 47

2.3.3 Teoria de Resposta Linear . . . . . . . . . . . . . . . 48

2.3.4 Resposta linear densidade-densidade . . . . . . . . . 52

2.3.5 As equações de Casida . . . . . . . . . . . . . . 55

2.4 DFTB . . . . . . . . . . . . . . 56

2.4.1 A variante SCC-DFTB . . . . . . . . . . 58

2.4.2 Energia da estrutura de banda e o pseudo-átomo . . . 59

2.4.3 Correção de segunda ordem . . . . . . . . . . . . . . 60

2.4.4 Energia de repulsão . . . . . . . . . . . . . . . . . 62

2.4.5 Princípio variacional . . . . . . . . . . . . . 63

2.4.6 Correção de longo alcance . . . . . . . . . . . . . . . 64

2.5 LC-TD-DFTB . . . . . . . . . . . . . . . . 67

2.5.1 Generalização para sistemas de camada aberta . . . . 67

2.5.2 Adaptação das equações de Casida . . . . . . . . . . 68

2.6 Modelos de solvatação implícita . . . . . . . . . . . . . . 71

2.6.1 Modelo contínuo polarizável (PCM) . . . . . . . . 72

2.7 Relação de Clausius-Mossotti . . . . . . . . . . . . . . . . 74

2.8 Ensemble nuclear de configurações . . . . . . . . . . . . 75 
3 Resultados $\quad 77$

3.1 Efeito das funções de base na estrutura eletrônica . . . . . . 77

3.1 .1 Geometria . . . . . . . . . . . . . . . 78

3.1.2 Estado fundamental e orbitais moleculares . . . . . . . 79

3.1.3 Estados excitados e espectros de absorção . . . . . . . 82

3.2 Otimização do parâmetro de longo alcance . . . . . . . . . . 85

3.2.1 Hidrocarbonetos poli-aromáticos . . . . . . . . . 86

3.2.2 DTP-IC-4Ph . . . . . . . . . . . . . . . . 93

3.2.3 PBDB-T . . . . . . . . . . . . . . . . . . 97

3.3 Comparação entre metodologias . . . . . . . . . . . . . . 98

3.4 Espectros de absorção . . . . . . . . . . . . . . . . . 101

3.4.1 Hidrocarbonetos poli-aromáticos . . . . . . . . . . . 102

3.4.2 DTP-IC-4Ph . . . . . . . . . . . . . . . . 104

3.4.3 PBDB-T . . . . . . . . . . . . . . . . . 108

3.5 Estudo do efeito de solvente em HPAs . . . . . . . . . . . . 112

3.6 Modelos para Células Solares . . . . . . . . . . . . . 115

3.6.1 Constantes dielétricas . . . . . . . . . . . . . 116

3.6.2 Energia de ligação do éxciton . . . . . . . . . . . . . . 120

3.6.3 Energia intramolecular de reorganização . . . . . . . . 121

4 Conclusões $\quad 124$

$\begin{array}{ll}\text { Referências } & 127\end{array}$

A Expansão de Born-Huang $\quad 141$

B Derivação das Equações de Kohn-Sham 144

B.1 Integral de Coulomb . . . . . . . . . . . . . . . . . . . 144

B.2 Potencial externo . . . . . . . . . . . . . . . . . 145

B.3 Energia cinética do gás de elétrons não interagentes . . . . . 145

B.4 Vínculo de ortogonalidade . . . . . . . . . . . . . . . . 147

C Elementos do funcional $\omega$ B97X-D 148

D Operador de evolução temporal $\quad 151$

E Cálculo da resposta linear $\quad 152$

F Representação de Lehmann da função de resposta linear 155

G Vínculos entre respostas lineares dos sistemas real e de Kohn-Sham 
H Derivação das equações de Casida

I Funcional de Energia no método DFTB3 168

J Procedimento auto-consistente do modelo de solvatação implícita

K Modelo de dispersão de Slater-Kirkwood

L Comparação entre geometrias

MEstados excitados dos HPAs

N Força de oscilador, seção de choque e polarizabilidade

O Validação do algoritmo de cálculo do volume molecular 


\section{Capítulo 1}

\section{Introdução}

Fortemente relacionada aos países em desenvolvimento, a expectativa de crescimento no consumo global de energia entre os anos 2018 e 2050 é de cerca de 50\%, chegando à produção de energia elétrica líquida de $44 \mathrm{TW}$ em 2050 [1]. Projeta-se que as fontes renováveis passem a deter metade de toda a geração de energia elétrica, com destaque para a energia solar chegando a 8.3 trilhões de $\mathrm{kWh}^{1}$ e compondo $38 \%$ de toda a produção renovável.

Dentre as fontes de energia solar, as células fotovoltaicas à base de materiais orgânicos, ainda que apresentem baixas eficiências quando comparadas às inorgânicas, se sobressaem do ponto de vista industrial e atingem eficiências de conversão de energia (PCE) em torno de $10 \%$ com do tempo de vida extrapolado em 22 anos [2]. Por meio da análise do ciclo de vida (LCA) [3], que leva em consideração critérios energéticos e ambientais, tipicamente, o uso de materiais orgânicos na composição das células implica em baixo custo de produção e portanto baixo tempo de retorno de energia (EPBT) [4], que quando atrelados à alta insolação e à baixa taxa de emissão de carbono [5] levam as células orgânicas à posição de destaque.

\subsection{Células solares emergentes}

Os dispositivos fotovoltaicos que buscam substituir as estruturas cristalinas convencionais à base de silício compõem o conjunto de tecnologias emergentes, entre as quais podemos citar as DSSC (Dye Sensitized Solar Cells), células de Perovskita, células à base de pontos quânticos e as orgânicas. O NREL (National Renewable Energy Laboratory) reúne os trabalhos de diversos grupos de pesquisa ao longo das últimas décadas e compara as eficiências por meio de um infográfico [6]. A Figura 1.1 traz o desempenho dos dispositivos renováveis nos últimos anos.

\footnotetext{
${ }^{1}$ Ao longo de todo o texto o padrão com ponto $(0.000)$ é utilizado ao invés do com vírgula $(0,000)$ para representar quantidades numéricas.
} 


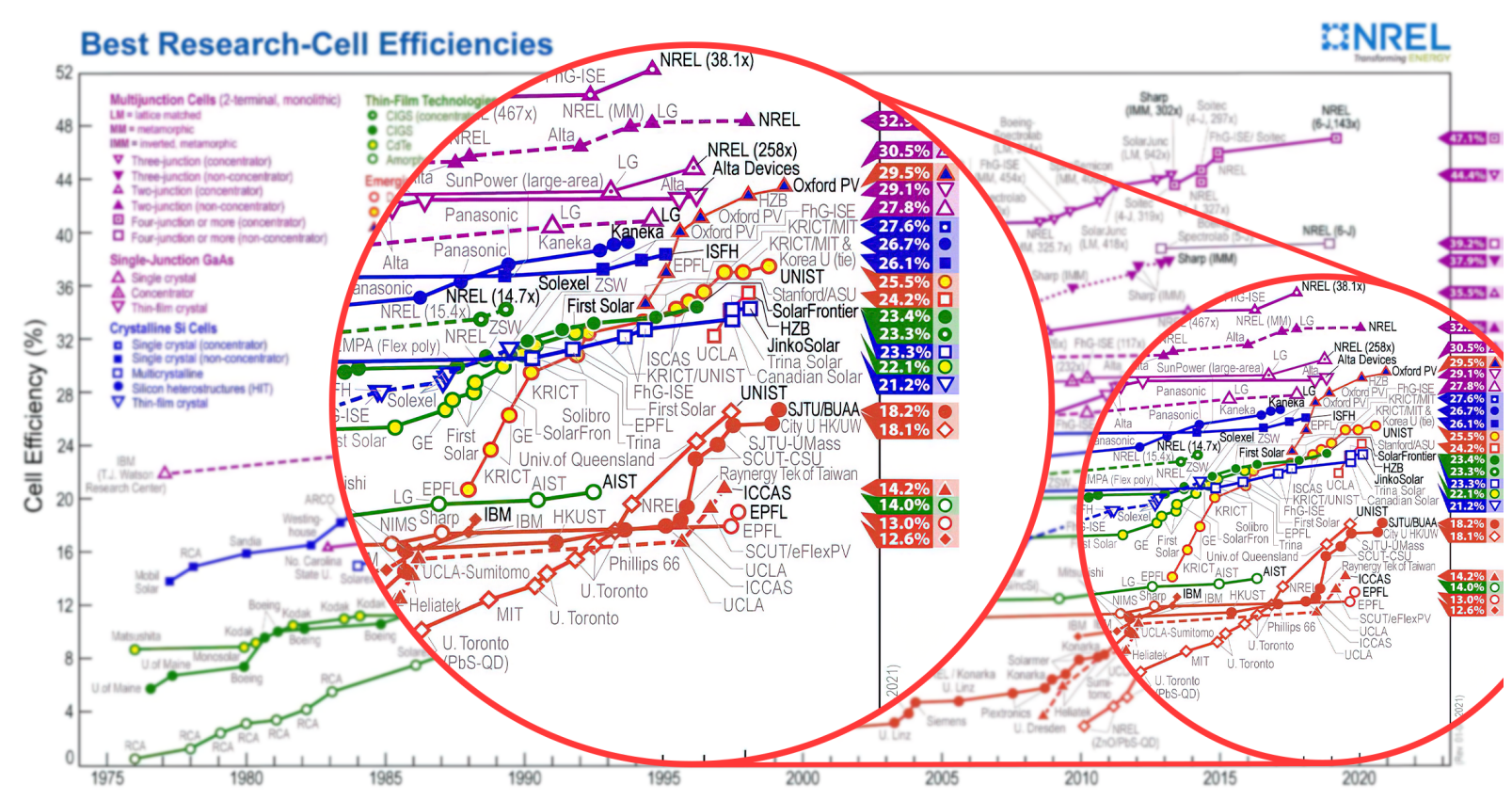

Figura 1.1: Adaptação do infográfico fornecido pela NREL [6] com a evolução da eficiência de diferentes células solares.

As linhas roxas, verdes e azuis representam células à base de compostos metálicos e as linhas laranjas dizem respeito às tecnologias emergentes, entre as quais as células de Perovskita se destacam, atingindo eficiências de $29.5 \%$. Ainda que alternativa, essa classe de dispositivos utiliza metais pesados e portanto não é uma fonte de energia limpa. Entre as fontes renováveis, as OSC (Organic Solar Cells) se destacam com eficiências superiores a $18 \%$ em oposição aos $13 \%$ das DSSC, fato este que quando associado ao crescimento nos últimos anos, fruto dos avanços no campo da engenharia de compostos, motiva o estudo e a caracterização no nível molecular dos doadores e aceitadores eletrônicos orgânicos.

A construção das OSC varia tanto na composição quanto na arquitetura dos dispositivos, mas de maneira geral podem ser representadas a partir da Figura 1.2. À esquerda são ilustradas as camadas que compõem a célula solar. Nas duas extremidades se encontram os contatos metálicos que fecham o circuito, junto a revestimentos que buscam minimizar a perda de radiação via reflexão. Em seguida, finas camadas de materiais condutores de elétrons (ou de buracos) auxiliam a movimentação dos portadores de carga. No centro se encontra a camada ativa, formada pelos compostos doadores e aceitadores, responsáveis pela absorção da radiação incidente e subsequente geração do par elétron-buraco que caracteriza a quasipartícula éxciton. 

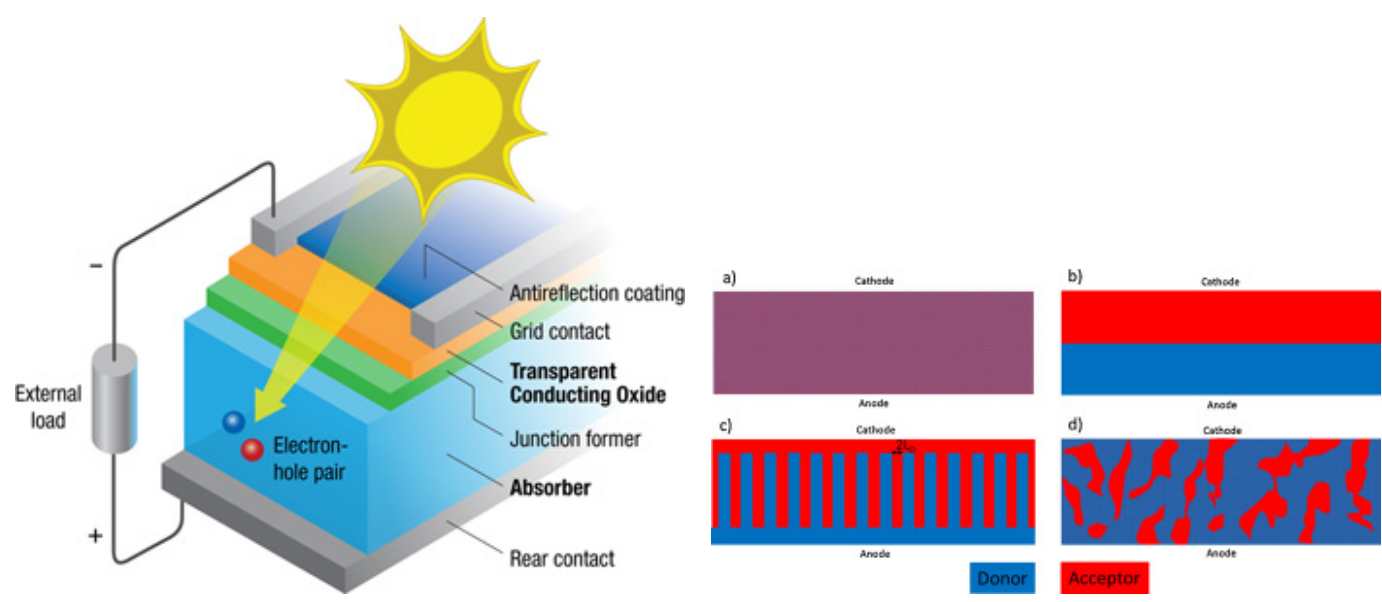

Figura 1.2: Representação esquemática das camadas de uma célula solar e do interior da camada ativa. Adaptado das referências [7, 8].

À direita na Figura 1.2, são apresentadas 4 representações do interior da camada ativa. A primeira delas (a), caracteriza uma distribuição homogênea dos compostos enquanto a (b) ilustra a disposição obtida por meio de técnicas de deposição à vácuo, que resultam na maior e menor superfície de contato possível entre doador e aceitador, respectivamente. Como na dinâmica de conversão de energia o éxciton deve chegar à interface D:A e, depois de dissociado, as partículas devem seguir em direção às extremidades (representadas na figura pelos eletrodos), o cenário (c) corresponde ao ideal, pois maximiza a superfície de contato efetiva sem a formação de ilhas de doador ou aceitador, que acabam por aprisionar as partículas no seu interior. A construção de tais ordenamentos é muito complicada e na prática a disposição que apresenta melhores resultados é a chamada heterojunção volumétrica, do inglês bulk heterojunction (BHJ), ilustrada no item (d).

No interior da camada ativa, a Figura 1.3 ilustra as etapas de conversão de energia solar em elétrica. Após a geração do éxciton, exemplificada pelo passo 1, no cenário ideal os pares vencem a competição frente aos processos de recombinação e difundem à interface entre doador e aceitador (D:A) (passo 2). Nessa interface, a relação entre as energias dos orbitais HOMOs e LUMOs favorece a dissociação das quasipartículas (passo 3) e gera portadores de carga livres tanto no caso em que o éxciton foi originado no doador (canal 1) quanto no aceitador (canal 2). Uma vez livres, os elétrons e buracos se movimentam de acordo com o campo elétrico externo (passo 4) até serem capturados nos eletrodos (passo 5), operando como um gerador. 


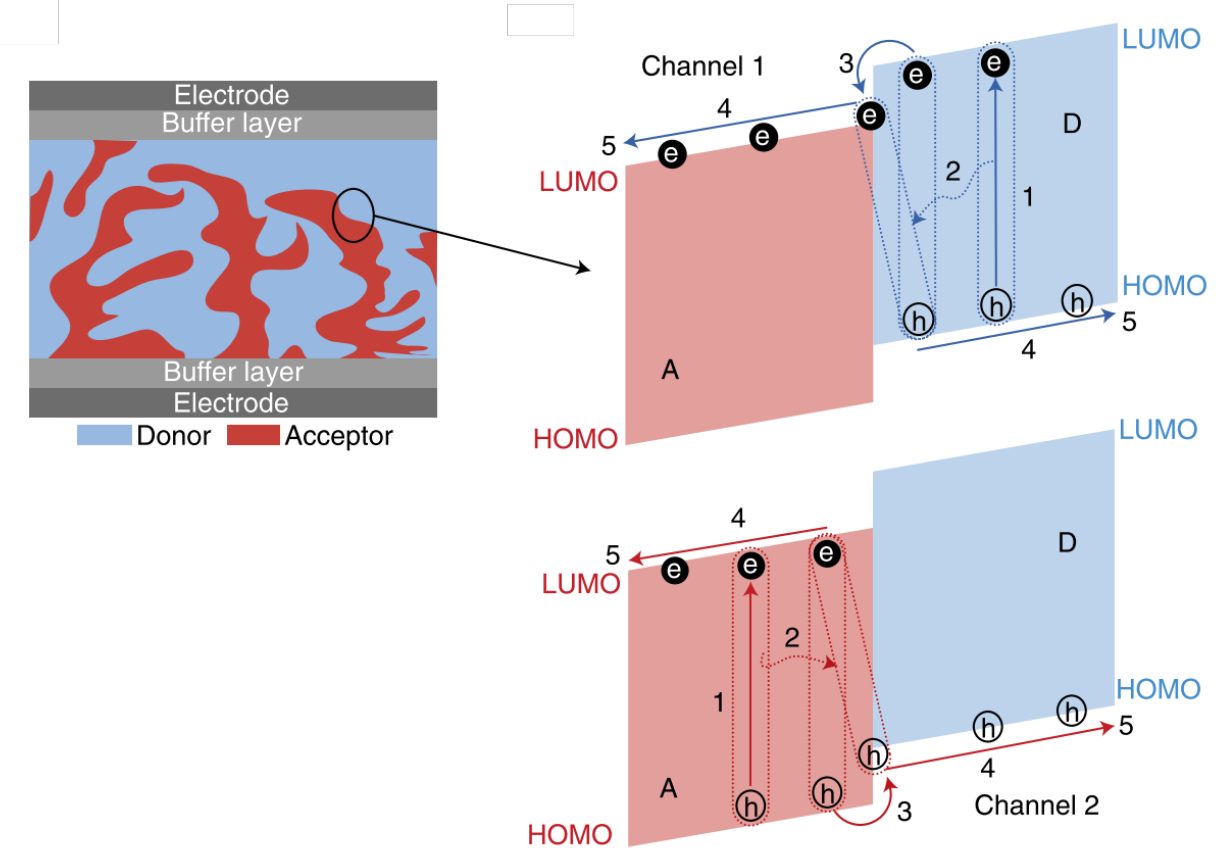

Figura 1.3: Seção transversal da camada ativa e as etapas da dinâmica interna das OSC. (1) Absorção de radiação e criação dos éxcitons; (2) difusão dos éxcitons até a interface D:A; (3) dissociação dos éxcitons; (4) movimentação dos portadores de carga em direção aos eletrodos; (5) extração dos portadores de carga. Adaptado da referência [9].

\subsection{As estruturas FREAs}

Nas últimas duas décadas houve um grande esforço em direção ao desenvolvimento de doadores com propriedades otimizadas [10], enquanto o uso de aceitadores se manteve dominado por fulerenos $\left(\mathrm{C}_{60}, \mathrm{C}_{70}\right)$ e seus derivados, sobretudo devido à alta capacidade de transporte de elétrons isotropicamente dada a simetria 3D dos compostos. Ainda que esses compostos tenham desempenhado um papel fundamental nos últimos anos, baixas absorções de luz na faixa do visível, limitações para calibrar o gap fundamental, difícil purificação e instabilidade morfológica [11, 12] acabam por conter avanços no desempenho das células solares. Nesse contexto, nos últimos anos investigações sobre NFAs (Non-Fullerene electron Acceptors) vem se destacando, ao ponto de motivar uma nova geração de OSC que ultrapassa o platô dos $11.5 \%$ de eficiência dos dispositivos com aceitadores a base de fulerenos [9]. Na Figura 1.4 são apresentados exemplos de NFAs. 


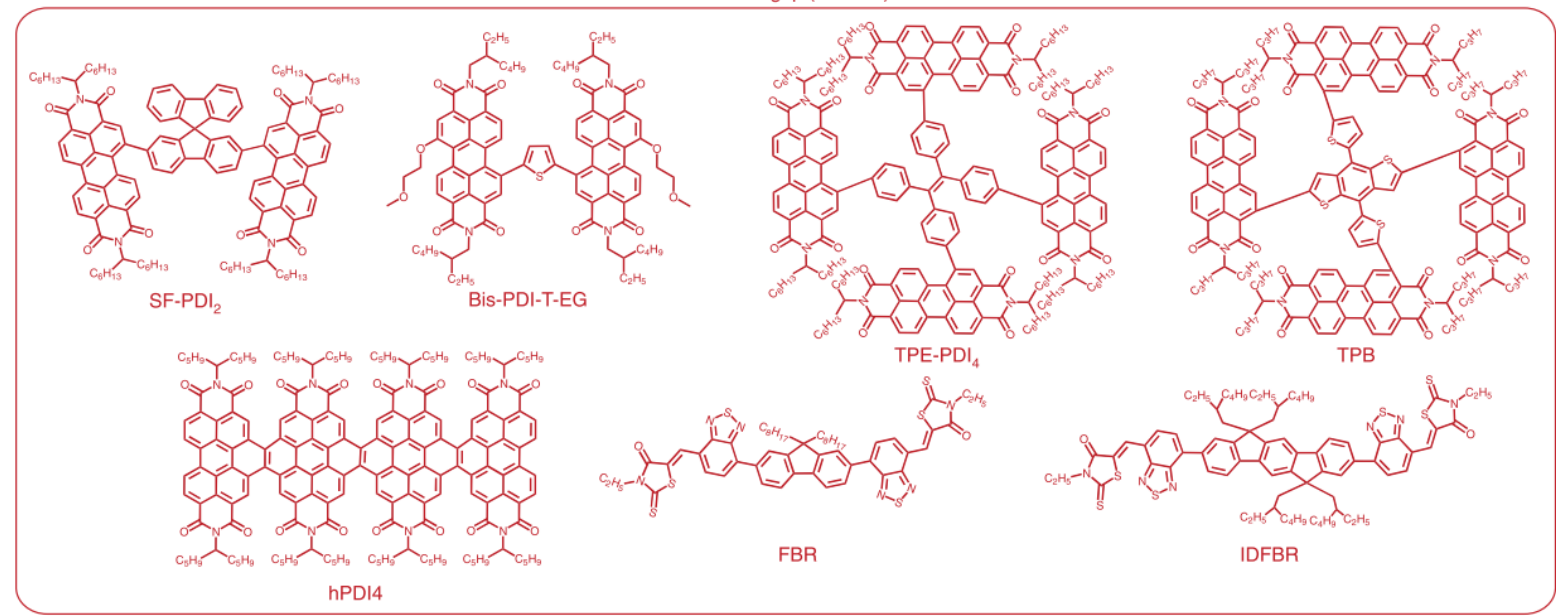

Medium bandgap (1.9-1.5 eV)

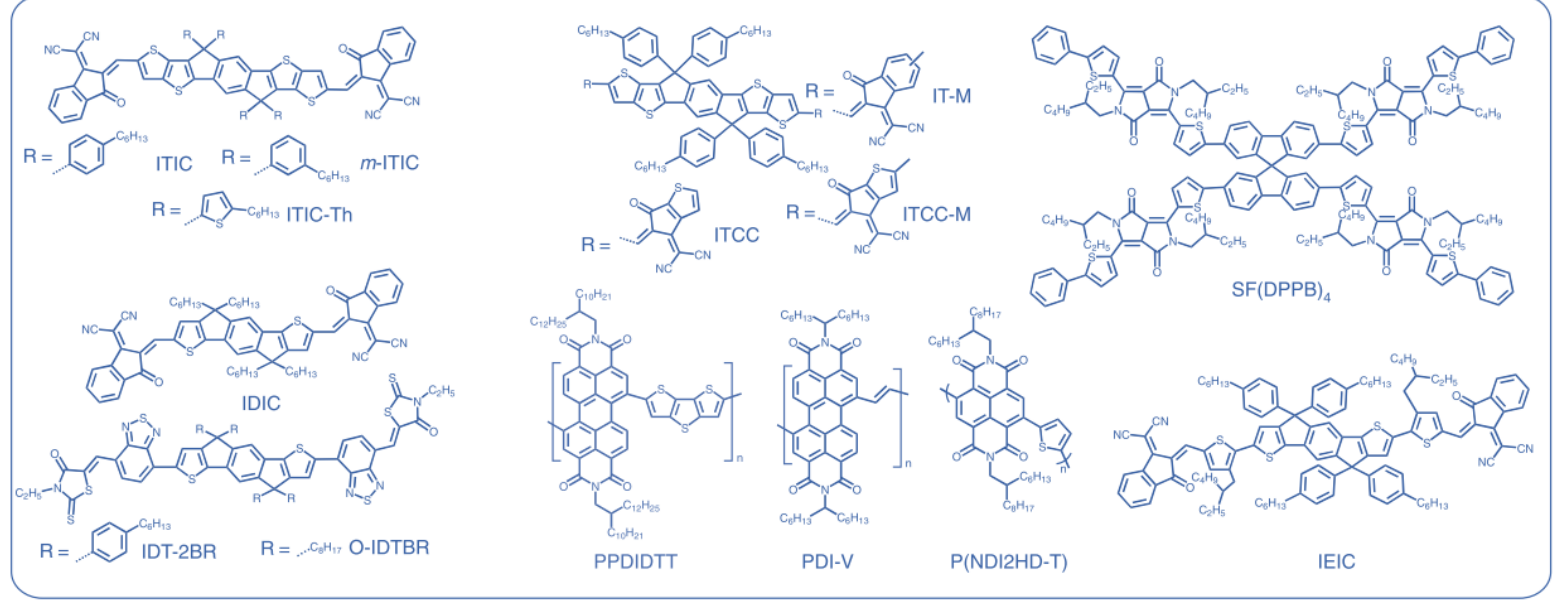

Narrow bandgap $(<1.5 \mathrm{eV})$

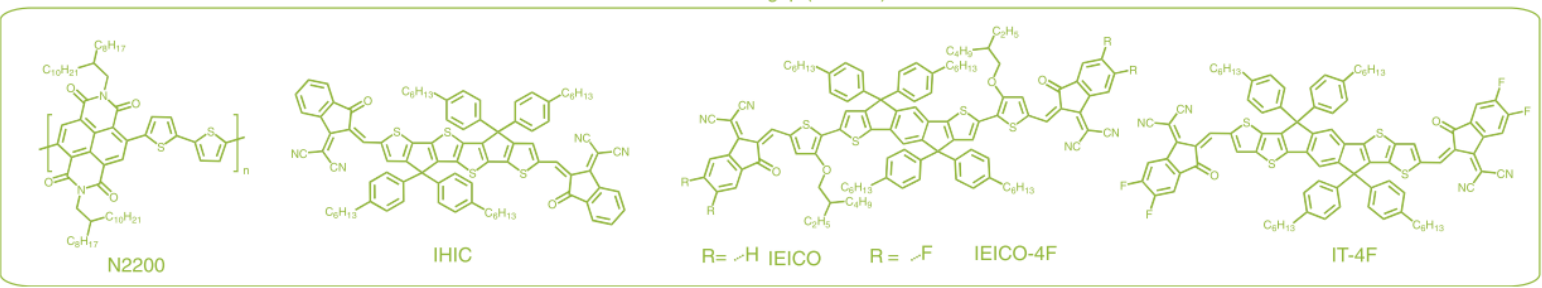

Figura 1.4: Estrutura química de exemplares NFAs com diferentes gaps de energia. Adaptado da referência [9].

Como a imagem sugere, a limitação dos fulerenos no que diz respeito ao gap de energia é contornada devido à grande flexibilidade na composição dos materiais. A alteração dos grupos funcionais otimiza a absorção de radiação na faixa do espectro de interesse assim como permite a calibração dos níveis de energia dos orbitais HOMO e LUMO. Essas alterações minimizam a competição por fótons entre aceitador e doador, uma vez que cada molécula acaba absorvendo predominantemente em comprimentos de onda distintos, e favorecem a dissociação do éxciton, dado o ganho energético da captação do elétron pelo material aceitador e do buraco pelo doador. Outras vantagens relacionadas à pureza, morfologia e estabilidade também 
estão presentes [13], mas por outro lado, a perda do perfil tridimensional resulta em anisotropias na topologia dos semicondutores não fulerênicos, o que pode comprometer a mobilidade dos portadores de carga [10].

Entre as alternativas aos fulerenos, Rylene diimides e FREAs (Fused-Ring Electron Acceptors) são duas classes de aceitadores que se destacam. A primeira é formada pela adição de átomos com alta eletronegatividade à estruturas $\pi$-conjugadas como o perileno $\left(\mathrm{C}_{20} \mathrm{H}_{12}\right)$, resultando em compostos como o SF-PDI 2 , hPDI4 e o N2200, apresentados na Figura 1.4. Os desempenhos desses aceitadores ilustram bem as vantagens e desvantagens discutidas [10]. A segunda classe foi introduzida por Zhan et al. em 2015 [14] e se sobressai em relação a primeira pois o red shift observado no espectro de absorção é mais intenso [10], o que tipicamente minimiza a intersecção com o espectro do doador. Em geral, essas moléculas são formadas a partir de um centro composto por anéis com caráter doador, ligado em ambos os lados a grupos com natureza aceitadora, caracterizando formações A-D-A, ilustradas na Figura 1.5. O resultado são estruturas com orbitais delocalizados que garantem maior transferência de carga intramolecular e consequentemente aumentam a mobilidade dos portadores de carga.

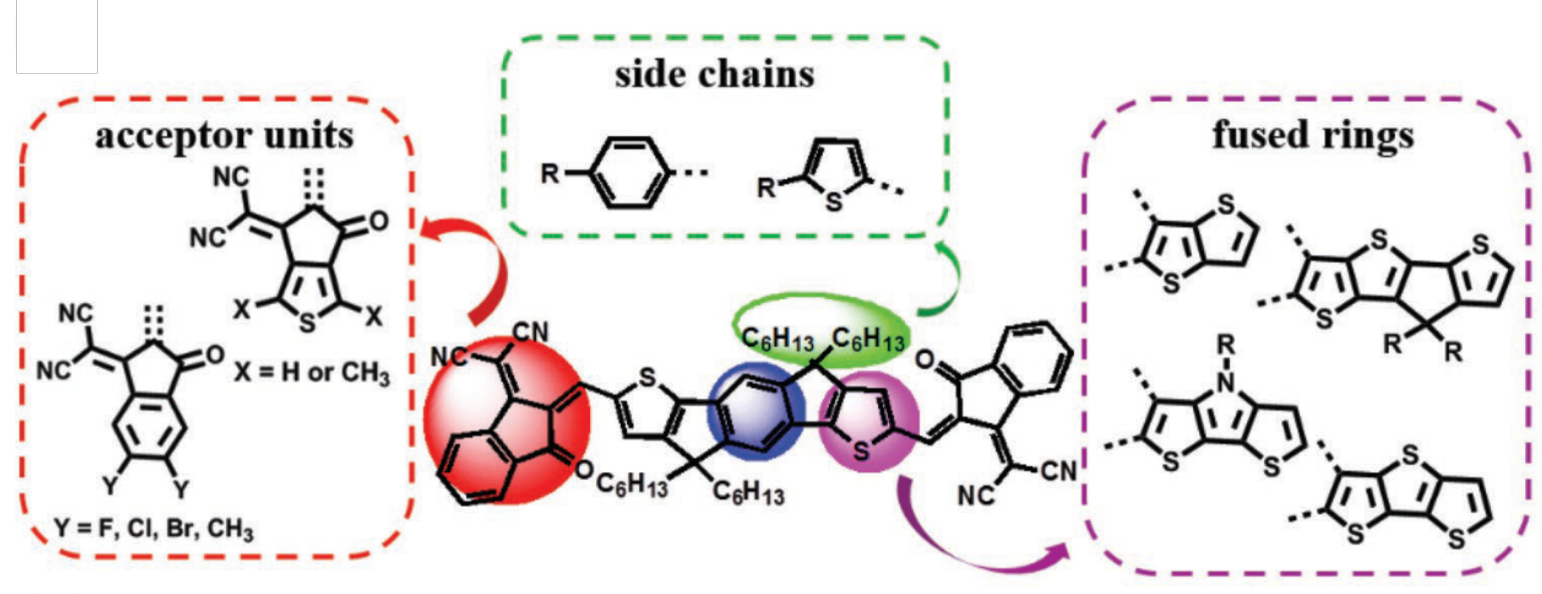

Figura 1.5: Representação de uma estrutura FREA. A figura foi adaptada da referência [15].

Os compostos IEIC, IHIC e IT-4F da Figura 1.4 são exemplos de FREAs e, em particular, quando comparamos a PCE de células solares que utilizam IHIC e N2200 combinados ao doador PBDB-T, os valores de $10.4 \%$ e $5.8 \%$ $[16,17]$, respectivamente, ilustram os desempenhos em média superiores dos FREAs. 


\subsection{O aceitador DTP-IC-4Ph}

O caminho explorado por Wang et al. [15] segue a tendência de desenvolver semicondutores orgânicos a partir de HPAs (Hidrocarbonetos Policíclicos Aromáticos). Essa classe de compostos é caracterizada por anéis cíclicos com alternância de ligações simples e duplas, formando sistemas $\pi$-conjugados com orbitais delocalizados que garantem altas absorbâncias e ótima mobilidade dos portadores de carga, dois aspectos fundamentais no contexto de células solares. No artigo dos pesquisadores chineses, o baixo custo de produção, a facilidade de adaptação estrutural e as propriedades optoeletrônicas altamente ajustáveis dos HPAs possibilitaram o desenvolvimento de uma nova classe de estruturas FREAs, os aceitadores DTx-IC-nPh, apresentados na Figura 1.6.

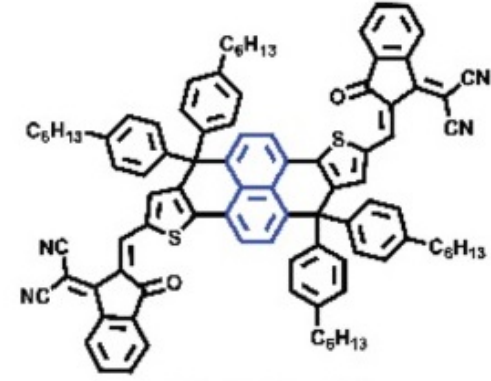

DTN-IC-2Ph

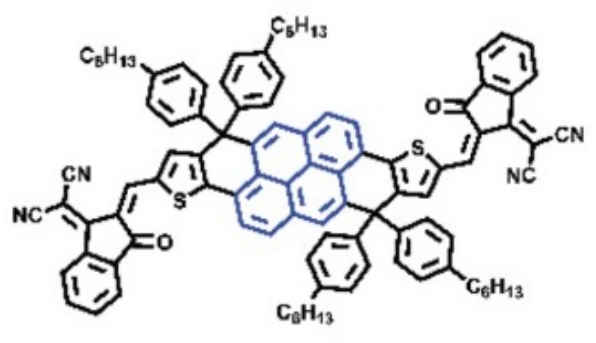

DTP-IC-4Ph

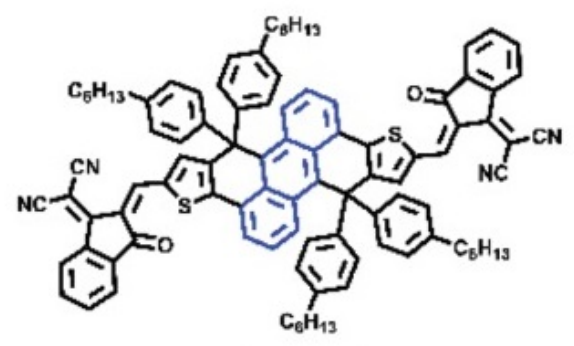

DTA-IC-3Ph

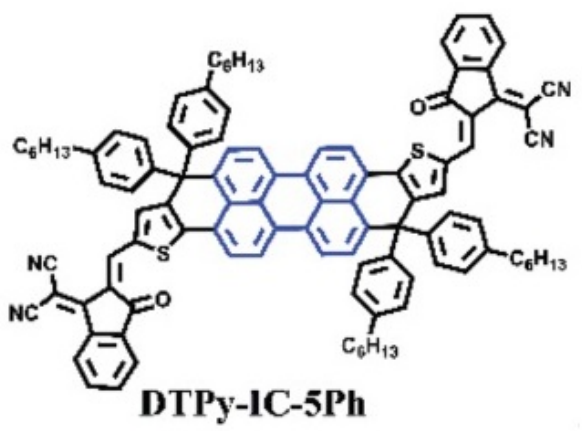

Figura 1.6: Figura com as representações em linha dos compostos desenvolvidos por Wang et al. [15].

As estruturas incorporam as moléculas de naftaleno (2 anéis), antraceno (3 anéis), pireno (4 anéis) e perileno ( 5 anéis), destacados em azul na Figura 1.6. A síntese é realizada de tal forma que os HPAs se unem à estrutura central, de modo que a única diferença entre cada aceitador é o número de anéis aromáticos. Experimentalmente foi observado um gradual desvio para o vermelho no espectro de absorção (red shift) e um aumento na absorbância à medida que o composto cresce. A diminuição na solubilidade 
e aumento das interações intermoleculares também foram observados, de tal forma que a presença de 4 anéis otimiza o conjunto de propriedades em questão. Como consequência, a PCE de $10.37 \%$ obtida para uma célula solar ${ }^{2}$ livre de aditivos com junção única de DTP-IC-4Ph:PBDB-T reproduz tais qualidades quando comparamos com a segunda melhor eficiência de $5.76 \%$ para o aceitador DTA-IC-3Ph na presença do mesmo doador PBDB-T. Os aditivos são utilizados para a otimização da morfologia da camada ativa, porém tipicamente possuem alto ponto de ebulição que impede a remoção adequada dos mesmos [18]. A presença residual desses aditivos, além de afetar a reprodutibilidade das células solares, compromete a estabilidade do dispositivo, promovendo a deterioração da performance. Nesse contexto, a família de aceitadores DTx-IC-nPh é favorável em termos de durabilidade e, em particular a PCE do par DTP-IC-4Ph:PBDB-T, se aproxima dos maiores valores obtidos para OSC com NFAs sem aditivos [18], se destacando entre as NFAs a base de HPAs (ver Tabela S1 de [15]).

A descrição realista das células solares é difícil por diversos fatores. A complexidade das moléculas que compõem a camada ativa é um gargalo do ponto de vista computacional, o que tende a restringir a aplicação de métodos ab initio. Sem contar efeitos do ambiente, da morfologia e até mesmo da temperatura [9], a determinação efetiva da mobilidade dos éxcitons está sujeita a absorção da radiação, expressa em termos da EQE (External Quantum Efficiency) ${ }^{3}$ [19], processos de recombinação radiativos e não-radiativos, que envolvem a emissão espontânea e estimulada de fótons, além do acoplamento via intersecções cônicas com o estado fundamental [20, 21]. Nesse cenário, ainda que idealizados, modelos atomísticos são essenciais para a compreensão dos fenômenos mencionados, seja por meio da determinação das energias dos orbitais [22], estudo da evolução temporal dos orbitais de transferência de carga [21] ou até mesmo caracterização da distribuição espacial entre doador e aceitador [23].

Tendo em vista os resultados experimentais dos compostos mostrados na Figura 1.6, é interessante investigar as propriedades dos HPAs utilizados e, devido ao alto custo computacional associado ao tratamento quântico dos aceitadores, se concentra no estudo da unidade DTP-IC-4Ph. O polímero utilizado como doador eletrônico também é estudado, desde sua origem em

\footnotetext{
${ }^{2}$ As condições do experimento foram a temperatura ambiente com massa de ar AM 1.5G e potência da radiação de $100 \mathrm{~mW} / \mathrm{cm}^{2}$.

${ }^{3} \mathrm{~A}$ EQE é definida como a razão do número de elétrons que chegam aos eletrodos pelo número de fótons incidentes.
} 
2015 [16] vem sendo pareado com diversos aceitadores [24, 25, 26, 27] e por meio de substituições de alguns grupos funcionais deu origem à uma família de doadores promissores que chegam a atingir eficiências de $17 \%$ em OSCs [27, 28, 29]. A Figura 1.7 ilustra alguns desses compostos.
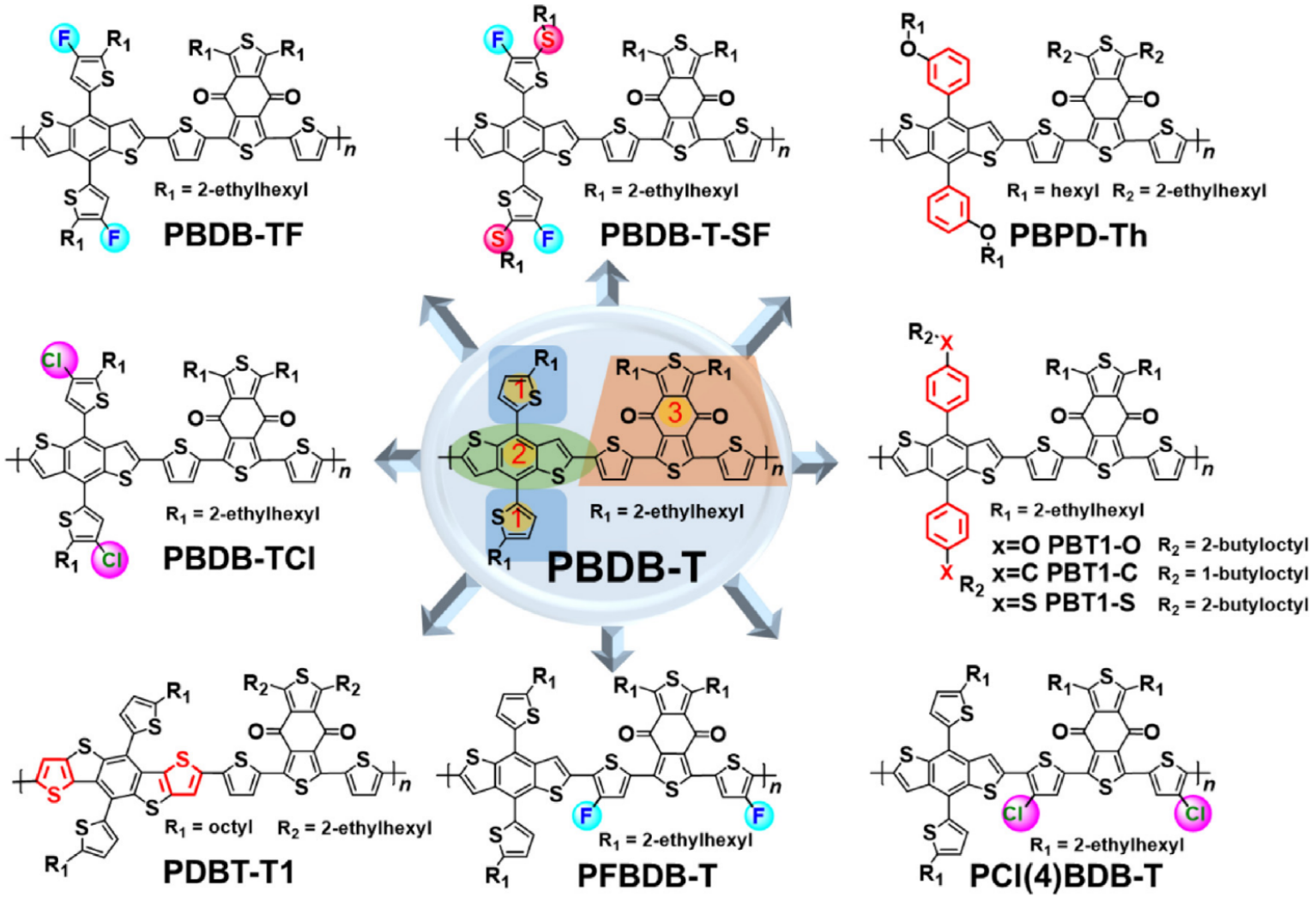

Figura 1.7: Representação do PBDB-T e de outros doadores derivados. Adaptação da referência [27].

Da mesma maneira que para o aceitador eletrônico, a caracterização do doador em nível molecular é essencial pois possibilita o refinamento da descrição dos dispositivos fotovoltaicos, complementando a análise e servindo de ferramenta para prever a viabilidade de determinada combinação D:A, por exemplo, por meio da comparação entre as energias dos orbitais HOMO e LUMO do doador e do aceitador.

\subsection{A dissertação e seus objetivos}

O texto é organizado em 4 partes, sendo a primeira delas a Introdução, que até o momento apresentou os compostos de interesse assim como suas aplicações, e agora busca esclarecer os objetivos do projeto. Em seguida, 
o Capítulo 2 traz os métodos utilizados nos cálculos. O primeiro tópico abordado é a aproximação de Born-Oppenheimer, um dos pilares da construção teórica que fundamenta o desenvolvimento dos métodos de estrutura eletrônica. Entre as metodologias utilizadas, a DFT (Density Functional Theory) e a TD-DFT (Time-Dependent Density Functional Theory) são introduzidas sob a luz dos teoremas de existência e unicidade, seguindo o formalismo de Kohn-Sham. Essas construções são apresentadas na literatura em diferentes níveis de profundidade [30, 31, 32, 33, 34], mas o cálculo das derivadas funcionais que compõem as deduções das equações de Kohn-Sham raramente é apresentado por completo como no Apêndice B. A base atômica e o funcional de correlação e troca utilizados nos cálculos via (TD)-DFT são apresentados em detalhe, assim como o desenvolvimento da Teoria de Resposta Linear, cujas passagens matemáticas são esmiuçadas ao longo dos Apêndices D, E, F, G e H, que possibilita a incorporação de efeitos dependentes do tempo.

A vertente semi-empírica LC-DFTB, que corresponde à principal ferramenta do projeto, é apresentada ao longo da Seção 2.4, retratando o formalismo com suas hipóteses e parametrizações, com destaque para a correção de longo alcance $[35,36]$ que vem aumentando a popularidade do método $[37,23,38,39]$ e é essencial para a descrição de processos de transferência de carga $[23,38]$. A versão dependente do tempo da DFTB utiliza do formalismo de resposta linear da TD-DFT e apenas as adaptações do modelo Tight-Binding são discutidas. Para encerrar a teoria, o texto discorre sobre a incorporação de efeitos do ambiente via modelo de solvatação implícita, a determinação das constantes dielétricas por meio da relação de ClausiusMossotti e o levantamento dos espectros de absorção a partir do ensemble de coordenadas nucleares.

Os resultados são apresentados no Capítulo 3 e partem do estudo da base atômica. Com o intuito de entender o que devemos esperar de funções de base maiores, cálculos (TD)-DFT com o funcional $\omega$ B97X-D são realizados para os HPAs (naftaleno, antraceno e pireno) e mudanças nas propriedades óticas e estruturais são investigadas, elucidando potenciais limitações decorrentes da utilização de base mínima na DFTB. Em seguida, a otimização do parâmetro de longo alcance é realizada a partir do Teorema de Janak, tanto para os hidrocarbonetos quanto para o DTP-IC-4Ph e o PBDB-T. O aceitador e o doador são caracterizados via LC-(TD-)DFTB com o conjunto OB2 (Parametrization for Organic and Biological Mole- 
cules) de parâmetros de Slater-Koster com a adição do átomo de enxofre $(\mathrm{OB} 2+\mathrm{S})$, enquanto para os HPAs são utilizados ambos os métodos (TD)-DFT/ $\omega$ B97X-D/6-31G(d,p) e LC-(TD-)DFTB/OB2+S, visando a validação da metodologia LC-TD-DFTB, discutida na Seção 3.3. O tratamento de polímeros envolve algumas sutilezas [40, 41, 42], e frente as limitações computacionais, apenas o monômero de PBDB-T é estudado no presente trabalho.

Na Seção 3.4 os espectros de absorção são determinados, dando continuidade à validação da LC-TD-DFTB via HPAs e permitindo, a partir da média de 1000 configurações nucleares sobre a região de Franck-Condon, a determinação da seção de choque de absorção do DTP-IC-4Ph e do PBDB$\mathrm{T}$, configurando um dos objetivos centrais do projeto. Esses resultados complementam as discussões do modelo e elucidam algumas restrições da caracterização de polímeros a partir de um único oligômero. Na última parte, o efeito do solvente em HPAs é explorado via (TD-)DFT/ $\omega$ B97XD/6-31G(d,p) no nível PCM, (Polarizable Continuum Model) e são determinadas as constantes dielétricas, energias de ligação dos éxcitons e energias intramoleculares de reorganização dos compostos de interesse, propriedades essas relacionadas às células solares. O cálculo das constantes dielétricas utiliza da polarizabilidade, cuja determinação e a conexão com as demais propriedades óticas é discutida no Apêndice $\mathrm{N}$, e do volume da molécula, o que levou ao desenvolvimento de um algoritmo via Método de Monte-Carlo para a determinação do mesmo. A validação do algoritmo é discutida no Apêndice O. Por fim, pontos importantes e resultados centrais são retomados na Seção 4, trazendo à tona as principais conclusões e arrematando o texto. 


\section{Capítulo 2}

\section{Metodologias}

Nessa seção serão apresentados os formalismos e modelos utilizados ao longo do projeto. A primeira parte introduz a aproximação BornOppenheimer buscando elucidar a validade da mesma, seguindo com os formalismos da DFT e DFTB com suas respectivas variantes dependentes do tempo. A caracterização do solvente a nível PCM é discutida, comentando a respeito do procedimento auto-consistente SCRF (Self-Consistent Reaction Field), assim como a determinação das constantes dielétricas via relação de Clausius-Mossotti e a abordagem NEA (Nuclear Ensemble Approach) para calcular espectros de absorção.

Durante a descrição da DFT são estabelecidos os teoremas de existência e unicidade que garantem a dedução das equações de Kohn-Sham a partir do princípio variacional. O funcional e a base atômica utilizados são discutidos com mais detalhes. Para a TDDFT a construção parte dos teoremas fundamentais e utiliza da Teoria de Resposta Linear para chegar nas equações matriciais de Casida. Nessas subseções as equações são demonstradas com maior detalhamento e boa parte das contas se encontram nos apêndices. Por se tratar de uma ramificação da (TD)DFT, a (TD)DFTB compartilha boa parte dos conceitos apresentados e portanto são discutidas apenas as aproximações e parametrizações realizadas.

\subsection{Aproximação Born-Oppenheimer}

Em decorrência da complexidade em torno da solução numérica da equação de Schrödinger para sistemas multieletrônicos, é natural o uso de aproximações. Considere a equação de Schrödinger independente do tempo,

$$
\hat{\mathcal{H}} \Psi=E \Psi
$$

onde $\hat{\mathcal{H}}$ corresponde ao operador hamiltoniano, $\Psi$ a função de onda e $E$ a energia. No caso não-relativístico de uma molécula poliatômica com $N$ 
elétrons e $N_{n}$ núcleos, adotando o sistema de unidades atômicas $\left(\hbar=m_{e}=\right.$ $\left.e=\left(4 \pi \epsilon_{0}\right)^{-1}=1\right)$, a hamiltoniana total do sistema é dada por [30],

$$
\begin{aligned}
\hat{\mathcal{H}}_{m o l}= & -\sum_{\alpha=1}^{N_{n}} \frac{\nabla_{\alpha}^{2}}{2 m_{\alpha}}-\sum_{i=1}^{N} \frac{\nabla_{i}^{2}}{2}-\sum_{\alpha=1}^{N_{n}} \sum_{i=1}^{N} \frac{Z_{\alpha}}{\left\|\boldsymbol{r}_{i}-\boldsymbol{R}_{\alpha}\right\|} \\
& +\frac{1}{2} \sum_{i \neq j}^{N_{e}} \frac{1}{\left\|\boldsymbol{r}_{i}-\boldsymbol{r}_{j}\right\|}+\frac{1}{2} \sum_{\alpha \neq \beta}^{N_{n}} \frac{Z_{\alpha} Z_{\beta}}{\left\|\boldsymbol{R}_{\alpha}-\boldsymbol{R}_{\beta}\right\|} \\
= & \hat{T}_{n}+\hat{T}_{e}+\hat{V}_{e n}+\hat{V}_{e e}+\hat{V}_{n n},
\end{aligned}
$$

sendo $m_{\alpha}, Z_{\alpha}$ e $\boldsymbol{R}_{\alpha}$, respectivamente, a massa, o número atômico, e o operador de posição do $\alpha$-ésimo núcleo, e $\boldsymbol{r}_{i}$ o operador de posição do $i$ ésimo elétron. As letras gregas indexam os núcleos e as romanas os elétrons, de tal forma que $\nabla_{\beta}^{2}$ corresponde ao laplaciano com relação as coordenadas do $\beta$-ésimo núcleo e $\nabla_{j}^{2}$ com relação as coordenadas do $j$-ésimo elétron. Dado o caráter distinto dos constituintes, é conveniente realizar a separação Born-Oppenheimer, que consiste em desmembrar a hamiltoniana em parte eletrônica e parte nuclear,

$$
\hat{\mathcal{H}}_{m o l}=\underbrace{\hat{T}_{n}+\hat{V}_{n n}}_{\hat{\mathcal{H}}_{n}}+\underbrace{\hat{T}_{e}+\hat{V}_{e n}+\hat{V}_{e e}}_{\hat{\mathcal{H}}_{e}} \equiv \hat{\mathcal{H}}_{n}+\hat{\mathcal{H}}_{e} .
$$

Como os núcleos são muito mais pesados, é coerente assumir que diante do tempo característico da dinâmica eletrônica eles se comportam como cargas puntiformes fixas, de tal forma que a função de onda eletrônica depende parametricamente das coordenadas nucleares,

$$
\hat{\mathcal{H}}_{e} \psi_{l}(\boldsymbol{r} \mid \boldsymbol{R})=\epsilon_{l}(\boldsymbol{R}) \psi_{l}(\boldsymbol{r} \mid \boldsymbol{R}),
$$

onde $\boldsymbol{r}$ e $\boldsymbol{R}$ denotam, respectivamente, o conjunto de coordenadas eletrônicas e nucleares, $\epsilon_{l}$ o l-ésimo autovalor de $\hat{\mathcal{H}}_{e}$ com seu respectivo autoestado $\psi_{l}(\boldsymbol{r} \mid \boldsymbol{R})$, dependendo explicitamente das coordenadas eletrônicas e parametricamente das nucleares. Desta forma, podemos realizar a expansão de Born-Huang que consiste em escrever a função de onda total como produto de funções de onda nuclear e eletrônica [43],

$$
\Psi(\boldsymbol{r}, \boldsymbol{R})=\sum_{k} \chi_{k}(\boldsymbol{R}) \psi_{k}(\boldsymbol{r} \mid \boldsymbol{R})
$$

Tendo em vista que a função de onda nuclear se comporta como escalar 
frente a operadores eletrônicos, ao substituir (2.4) em (2.1) considerando a hamiltoniana molecular (2.2) e projetar no $l$-ésimo autoestado eletrônico (ver Apêndice A), obtemos que,

$$
\sum_{\alpha=1}^{N_{n}}\left(-\frac{\nabla_{\alpha}^{2}}{2 m_{\alpha}}-\frac{\left(\nabla_{\alpha}^{2}\right)_{l l}}{2 m_{\alpha}}+\left(\hat{\mathcal{H}}_{e}\right)_{l l}-\mathcal{E}(\boldsymbol{R})\right) \chi_{l}(\boldsymbol{R})=\sum_{\alpha=1}^{N_{n}} \sum_{k \neq l}\left(\frac{\left(\nabla_{\alpha}^{2}\right)_{l k}}{2 m_{\alpha}}+\frac{\left(\nabla_{\alpha}\right)_{l k} \cdot \nabla_{\alpha}}{m_{\alpha}}\right) \chi_{k}(\boldsymbol{R}) .
$$

Ainda que o conjunto de equações acima determine $\chi_{k}(\boldsymbol{R})$, o acoplamento entre estados nucleares e eletrônicos inviabiliza a solução numérica. Entretanto, no caso em que os termos não diagonais são desprezíveis,

$$
\sum_{\alpha=1}^{N_{n}}\left(-\frac{\nabla_{\alpha}^{2}}{2 m_{\alpha}}-\frac{\left(\nabla_{\alpha}^{2}\right)_{l l}}{2 m_{\alpha}}+\left(\hat{\mathcal{H}}_{e}\right)_{l l}\right) \chi_{l}(\boldsymbol{R})=\mathcal{E}(\boldsymbol{R}) \chi_{l}(\boldsymbol{R})=0 .
$$

Essa abordagem é chamada de aproximação adiabática e permite a interpretação de $\chi_{l}(\boldsymbol{R})$ como autoestados nucleares associados a autoestados eletrônicos. Pela relação (2.3), os termos eletrônicos diagonais são simplesmente os autovalores eletrônicos, de tal forma que ao desprezar a correção diagonal da energia cinética nuclear, conclui-se a aproximação de BornOppenheimer [44], resultando em,

$$
\sum_{\alpha=1}^{N_{n}}\left(-\frac{\nabla_{\alpha}^{2}}{2 m_{\alpha}}+\epsilon_{l}(\boldsymbol{R})\right) \chi_{l}(\boldsymbol{R})=\mathcal{E}(\boldsymbol{R}) \chi_{l}(\boldsymbol{R}) .
$$

A energia eletrônica $\epsilon_{l}(\boldsymbol{R})$ é também chamada de superfície de energia potencial Born-Oppenheimer pois se comporta como energia potencial do movimento nuclear.

\section{Validade da aproximação Born-Oppenheimer}

A partir da equação acoplada (2.5), verifica-se que a magnitude dos termos não diagonais está diretamente relacionada a $\left(\nabla_{\alpha}\right)_{l k}$, uma vez que os elementos de matriz do laplaciano obedecem a relação [45],

$$
\left(\nabla_{\alpha}^{2}\right)_{n m}=\left[\left(\nabla_{\alpha}\right)_{n m}\right]^{2}+\nabla_{\alpha} \cdot\left(\nabla_{\alpha}\right)_{n m} .
$$

Tomando o gradiente na equação (2.3), segue que,

$$
\nabla_{\alpha}\left(\hat{\mathcal{H}}_{e} \psi_{l}(\boldsymbol{r} \mid \boldsymbol{R})\right)=\epsilon_{l}(\boldsymbol{R})\left[\nabla_{\alpha} \psi_{l}(\boldsymbol{r} \mid \boldsymbol{R})\right]+\left[\nabla_{\alpha} \epsilon_{l}(\boldsymbol{R})\right] \psi_{l}(\boldsymbol{r} \mid \boldsymbol{R})
$$


Multiplicando ambos os lados por $\psi_{k}(\boldsymbol{r} \mid \boldsymbol{R})^{*}$ e integrando sobre todas as coordenadas eletrônicas,

$$
\int d \boldsymbol{r}\left[\psi_{k}^{*}\left(\nabla_{\alpha} \hat{\mathcal{H}}_{e}\right) \psi_{l}+\psi_{k}^{*} \hat{\mathcal{H}}_{e}\left(\nabla_{\alpha} \psi_{l}\right)\right]=\epsilon_{l}(\boldsymbol{R}) \int d \boldsymbol{r} \psi_{k}^{*} \nabla_{\alpha} \psi_{l}+\nabla_{\alpha} \epsilon_{l}(\boldsymbol{R}) \int d \boldsymbol{r} \psi_{k}^{*} \psi_{l} .
$$

Como $\hat{\mathcal{H}}_{e}$ é um operador hermitiano e $k \neq l$, verifica-se que,

$$
\left(\nabla_{\alpha}\right)_{l k}=\frac{\int d \boldsymbol{r} \psi_{k}^{*}(\boldsymbol{r} \mid \boldsymbol{R})\left(\nabla_{\alpha} \hat{\mathcal{H}}_{e}\right) \psi_{l}(\boldsymbol{r} \mid \boldsymbol{R})}{\epsilon_{l}(\boldsymbol{R})-\epsilon_{k}(\boldsymbol{R})} \equiv \frac{\left\langle\psi_{k}\left|\nabla_{\alpha} \hat{\mathcal{H}}_{e}\right| \psi_{l}\right\rangle}{\epsilon_{l}(\boldsymbol{R})-\epsilon_{k}(\boldsymbol{R})} .
$$

Pela expressão acima fica evidente que quanto maior o espaçamento entre os estados eletrônicos melhor a qualidade da aproximação, sendo tipicamente adequada para descrever o estado fundamental do sistema.

\subsection{Teoria do Funcional de Densidade}

No que diz respeito a descrição de compostos moleculares e sistemas cristalinos, a Teoria do Funcional de Densidade vem sendo uma das metodologias mais utilizadas ao longo das últimas décadas [46]. Como o nome sugere, sua abordagem envolve o estudo da densidade eletrônica $\rho(\boldsymbol{r}, t)$, e em diversos casos proporciona resultados com elevada precisão a baixo custo computacional.

Ainda que densidades eletrônicas já houvessem sido objeto de estudo no passado, foi em 1927 que o modelo de Thomas-Fermi iniciou a discussão sobre a distribuição de elétrons em átomos. De maneira independente, os dois pesquisadores utilizaram a energia cinética de um gás de elétrons nãointeragentes $T[\rho(\boldsymbol{r})]$ e modelaram a energia de interação eletrônica $V_{e e}[\rho(\boldsymbol{r})]$ como o termo de Coulomb,

$$
V_{e e}[\rho(\boldsymbol{r})] \equiv J[\rho(\boldsymbol{r})]=\frac{1}{2} \iint d \boldsymbol{r}_{1} d \boldsymbol{r}_{2} \frac{\rho\left(\boldsymbol{r}_{1}\right) \rho\left(\boldsymbol{r}_{2}\right)}{\left\|\boldsymbol{r}_{2}-\boldsymbol{r}_{1}\right\|},
$$

que corresponde a interação eletrostática clássica entre duas distribuições de carga $\rho\left(\boldsymbol{r}_{1}\right)$ e $\rho\left(\boldsymbol{r}_{2}\right)$, para determinar a energia total do sistema. A vantagem de descrever um sistema de $N$ elétrons a partir de 3 componentes espaciais ao invés de $3 N$ variáveis mais termos de spin é imediata, porém apenas cerca de 40 anos depois foram estabelecidos os teoremas que 
possibilitaram a construção da teoria da forma como é hoje utilizada.

\section{Densidade eletrônica}

Considerando a hamiltoniana molecular (2.3) na aproximação de BornOppenheimer, o termo cinético nuclear é nulo, de tal forma que a menos de uma constante, $\hat{\mathcal{H}}_{\text {mol }}=\hat{\mathcal{H}}_{e}$. É conveniente reescrever da forma,

$$
\hat{\mathcal{H}}_{e}=\hat{T}_{e}+\hat{V}_{e n}+\hat{W}=-\sum_{i \neq j}^{N} \frac{\nabla_{i}^{2}}{2}+\sum_{i=1}^{N} v\left(\boldsymbol{r}_{i}\right)+\frac{1}{2} \sum_{i \neq j}^{N} w\left(\left\|\boldsymbol{r}_{i}-\boldsymbol{r}_{j}\right\|\right)
$$

onde $\hat{W} \equiv \hat{V}_{e e}, v\left(\boldsymbol{r}_{i}\right)$ corresponde ao potencial externo atuando no $i$-ésimo elétron e $w\left(\left\|\boldsymbol{r}_{i}-\boldsymbol{r}_{j}\right\|\right)$ a interação entre os elétrons, tipicamente Coulombiana, $w\left(\left\|\boldsymbol{r}_{i}-\boldsymbol{r}_{j}\right\|\right)=\left\|\boldsymbol{r}_{i}-\boldsymbol{r}_{j}\right\|^{-1}$. Neste contexto, a densidade de probabilidade do sistema estar no $j$-ésimo estado é dada por,

$$
\rho_{j}(\boldsymbol{r})=\sum_{\sigma}\left\langle\Psi_{j}\left|\sum_{i=1}^{N} \delta\left(\boldsymbol{r}_{i}-\boldsymbol{r}\right)\right| \Psi_{j}\right\rangle=N \sum_{\sigma} \int d \boldsymbol{x}_{2} \ldots \int d \boldsymbol{x}_{N}\left|\Psi_{j}\left(\boldsymbol{r}, \sigma, \boldsymbol{x}_{2}, \ldots, \boldsymbol{x}_{N}\right)\right|^{2},
$$

onde $\boldsymbol{r}=(x, y, z)$ é o vetor de posição, $\boldsymbol{x}_{i} \equiv\left(\boldsymbol{r}_{i}, \sigma_{i}\right)$ corresponde ao par de coordenadas espaciais do $i$-ésimo elétron com a respectiva projeção de spin $\sigma_{i}$, e a soma é dada sobre todas as projeções de spin. Por simplicidade tomaremos estados do tipo singleto, eliminando o somatório nas projeções de spin. O valor esperado de $\hat{V}_{\text {en }}$ por sua vez, é dado por,

$$
\left\langle\Psi\left|\hat{V}_{e n}\right| \Psi\right\rangle=\sum_{i=1}^{N} \int d \boldsymbol{r}_{1} \ldots \int d \boldsymbol{r}_{N} \Psi^{*}\left(\boldsymbol{r}_{1}, \ldots, \boldsymbol{r}_{N}\right) v\left(\boldsymbol{r}_{i}\right) \Psi\left(\boldsymbol{r}_{1}, \ldots, \boldsymbol{r}_{N}\right) .
$$

Utilizando a propriedade de filtragem da delta de Dirac,

$$
\int_{R} d \boldsymbol{x} f(\boldsymbol{x}) \delta\left(\boldsymbol{x}-\boldsymbol{x}_{0}\right)=\left\{\begin{array}{l}
f\left(\boldsymbol{x}_{0}\right), \text { se } \boldsymbol{x}_{0} \text { pertencer a região de integração } R \\
0, \text { caso contrário }
\end{array}\right.
$$

segue que, 


$$
\begin{aligned}
\left\langle\Psi\left|\hat{V}_{e n}\right| \Psi\right\rangle & =\sum_{i=1}^{N} \int d \boldsymbol{r} \int d \boldsymbol{r}_{1} \ldots \int d \boldsymbol{r}_{i} v(\boldsymbol{r}) \delta\left(\boldsymbol{r}-\boldsymbol{r}_{i}\right) \ldots \int d \boldsymbol{r}_{N} \Psi^{*} \Psi \\
& =\int d \boldsymbol{r} \rho(\boldsymbol{r}) v(\boldsymbol{r}) .
\end{aligned}
$$

Além disso, são impostas as condições de contorno físicas,

$$
\lim _{r \rightarrow \infty} \rho(\boldsymbol{r})=0 \quad \text { e } \int d \boldsymbol{r} \rho(\boldsymbol{r})=N,
$$

que correspondem, respectivamente, a finitude espacial da densidade, possibilitando a normalização da função de onda, e o vínculo de que a integral da densidade ao longo de todo o espaço deve corresponder ao número de elétrons do sistema. Assim, os valores esperados são tratados como funcionais de $\rho_{j}(\boldsymbol{r})$, com particular interesse no estado fundamental $(j=0)$ devido ao tratamento variacional a ser praticado.

\subsubsection{Teorema de Hohenberg-Kohn}

Um dos pilares do método corresponde ao mapeamento bijetivo entre a densidade e o potencial externo. Este resultado corresponde ao Teorema de Hohenberg-Kohn e é enunciado abaixo.

Teorema de HK: Em um sistema finito de $N$ elétrons interagentes, com uma dada interação partícula-partícula, $w\left(\left\|\boldsymbol{r}_{i}-\boldsymbol{r}_{j}\right\|\right)$, existe uma correspondência única entre o potencial externo $v(\boldsymbol{r})$ e a densidade do estado fundamental $\rho_{0}(\boldsymbol{r})$. Ou seja, o potencial externo é um funcional univocamente determinado pela densidade de probabilidade do estado fundamental, $v\left[\rho_{0}\right](\boldsymbol{r})$, sujeito apenas a uma constante aditiva arbitrária.

Demonstração. Considere dois potenciais externos $v(\boldsymbol{r})$ e $v^{\prime}(\boldsymbol{r})$ que geram a mesma densidade de probabilidade do estado fundamental, $\rho_{0}(\boldsymbol{r})$, porém oriunda de duas funções de onda distintas $\Psi_{0}$ e $\Psi_{0}^{\prime}$, respectivamente. Pelo princípio variacional, por um lado temos que, 


$$
\begin{aligned}
E_{0}\left[\rho_{0}\right] & <\left\langle\Psi_{0}^{\prime}\left|\hat{\mathcal{H}}_{e}\right| \Psi_{0}^{\prime}\right\rangle=\left\langle\Psi_{0}^{\prime}\left|\hat{\mathcal{H}}_{e}^{\prime}\right| \Psi_{0}^{\prime}\right\rangle+\left\langle\Psi_{0}^{\prime}\left|\left(\hat{\mathcal{H}}_{e}-\hat{\mathcal{H}}_{e}^{\prime}\right)\right| \Psi_{0}^{\prime}\right\rangle \\
& =E_{0}^{\prime}\left[\rho_{0}\right]+\int d \boldsymbol{r} \rho_{0}(\boldsymbol{r})\left[v(\boldsymbol{r})-v^{\prime}(\boldsymbol{r})\right]
\end{aligned}
$$

e pelo outro,

$$
\begin{aligned}
E_{0}^{\prime}\left[\rho_{0}\right] & <\left\langle\Psi_{0}\left|\hat{\mathcal{H}}_{e}^{\prime}\right| \Psi_{0}\right\rangle=\left\langle\Psi_{0}\left|\hat{\mathcal{H}}_{e}\right| \Psi_{0}\right\rangle+\left\langle\Psi_{0}\left|\left(\hat{\mathcal{H}}_{e}^{\prime}-\hat{\mathcal{H}}_{e}\right)\right| \Psi_{0}\right\rangle \\
& =E_{0}\left[\rho_{0}\right]+\int d \boldsymbol{r} \rho_{0}(\boldsymbol{r})\left[v^{\prime}(\boldsymbol{r})-v(\boldsymbol{r})\right]
\end{aligned}
$$

Somando (2.11) com (2.12) somos levados ao absurdo,

$$
E_{0}\left[\rho_{0}\right]+E_{0}^{\prime}\left[\rho_{0}\right]<E_{0}\left[\rho_{0}\right]+E_{0}^{\prime}\left[\rho_{0}\right]
$$

Assim verificamos a unicidade do potencial externo dada a densidade do estado fundamental não degenerada. A generalização no caso degenerado é direta [47]. A partir da formulação acima, é possível decompor o funcional da energia de tal forma que uma de suas parcelas dependa apenas do número de elétrons,

$$
\begin{aligned}
E_{0}\left[\rho_{0}\right] & =\left\langle\Psi_{0}\left|\hat{T}_{e}+\hat{V}_{e n}+\hat{W}\right| \Psi_{0}\right\rangle \\
& =\left\langle\Psi_{0}\left|\hat{T}_{e}+\hat{W}\right| \Psi_{0}\right\rangle+\left\langle\Psi_{0}\left|\hat{V}_{e n}\right| \Psi_{0}\right\rangle \\
& \equiv F\left[\rho_{0}\right]+\left\langle\Psi_{0}\left|\hat{V}_{e n}\right| \Psi_{0}\right\rangle
\end{aligned}
$$

onde $F[\rho]$ independe das coordenadas nucleares e é chamado funcional universal. Como consequência direta do teorema, são concebidos dois corolários. O primeiro é também conhecido como Segundo Teorema de Hohenberg-Kohn e estabelece a relação que possibilita a análise a partir de diferentes densidades. 
Corolário 1: Para qualquer densidade $\rho^{\prime}$ que satisfaça as condições de contorno (2.10), segue que,

$$
E_{0} \leq E\left[\rho^{\prime}\right]
$$

sendo válida a igualdade apenas para a densidade exata, $\rho_{0}$.

Utilizando da formulação introduzida acima e aplicando o método variacional,

$$
E_{0}\left[\rho^{\prime}\right]=F\left[\rho^{\prime}\right]+\left\langle\Psi\left[\rho^{\prime}\right]\left|\hat{V}_{e n}\right| \Psi\left[\rho^{\prime}\right]\right\rangle>F\left[\rho_{0}\right]+\left\langle\left|\Psi\left[\rho_{0}\right]\right| \hat{V}_{e n}\left|\Psi\left[\rho_{0}\right]\right|\right\rangle=E_{0}\left[\rho_{0}\right]
$$

onde $\left|\Psi\left[\rho^{\prime}\right]\right\rangle$ e $\left|\Psi\left[\rho_{0}\right]\right\rangle$ correspondem aos estados originados a partir das densidades $\rho^{\prime}$ e $\rho_{0}$, respectivamente. Tendo em vista que o estado fundamental corresponde a um ponto estacionário, o segundo corolário possibilita a determinação de $\rho_{0}$ através da equação de Euler-Lagrange,

$$
\frac{\delta}{\delta \rho(\boldsymbol{r})}\left\{E_{0}-\mu\left(\int d^{3} r^{\prime} \rho\left(\boldsymbol{r}^{\prime}\right)-N\right)\right\}=0,
$$

onde $\boldsymbol{r}^{\prime}=\left(x^{\prime}, y^{\prime}, z^{\prime}\right)$ e $\mu$ corresponde ao multiplicador de Lagrange associado ao número de elétrons fixo que, fisicamente, é interpretado como potencial químico, que diz respeito a energia em trânsito decorrente da variação do número de partículas do sistema.

Corolário 2: Sejam $\mu$ o potencial químico decorrente do número de elétrons fixo, $F[\rho]$ o funcional universal e $v_{0}(\boldsymbol{r})$ o potencial externo gerado pela disposição dos núcleos no estado eletrônico fundamental, segue que,

$$
\frac{\delta F[\rho]}{\delta \rho(\boldsymbol{r})}+v_{0}(\boldsymbol{r})=\mu
$$

Uma vez que $\mu$ é ajustado de forma a manter o número de elétrons fixo em $N$ (2.10), é traçado o mecanismo de obtenção da densidade eletrônica do estado fundamental por meio da equação acima. Entretanto, $F[\rho]$ é desconhecido, o que leva o desenvolvimento da teoria a ser centrado em sua modelagem, explorando propriedades exatas e melhores descrições dos termos cinético e de interação eletrônica. 


\subsubsection{Equações de Kohn-Sham}

Os primeiros passos em direção a resolução prática do problema foram propostos por meio da caracterização de um sistema fictício não interagente que por construção reproduz a mesma densidade eletrônica do estado fundamental do sitema real. Em 1965, os pesquisadores W. Kohn e L. J. Sham propuseram como função de onda total, $\Psi_{j}\left(\boldsymbol{r}_{1}, \boldsymbol{r}_{2}, \ldots, \boldsymbol{r}_{N}, \sigma\right)$, o determinante de Slater,

$$
\Psi\left(\boldsymbol{r}_{1}, \ldots, \boldsymbol{r}_{N}\right)=\frac{1}{\sqrt{N !}}\left|\begin{array}{cccc}
\phi_{1}\left(\boldsymbol{r}_{1}\right) \alpha(1) & \phi_{1}\left(\boldsymbol{r}_{2}\right) \alpha(2) & \ldots & \phi_{1}\left(\boldsymbol{r}_{N}\right) \alpha(N) \\
\phi_{1}\left(\boldsymbol{r}_{1}\right) \beta(1) & \phi_{1}\left(\boldsymbol{r}_{2}\right) \beta(2) & \ldots & \phi_{1}\left(\boldsymbol{r}_{N}\right) \beta(N) \\
\phi_{2}\left(\boldsymbol{r}_{1}\right) \alpha(1) & \phi_{2}\left(\boldsymbol{r}_{2}\right) \alpha(2) & \ldots & \phi_{2}\left(\boldsymbol{r}_{N}\right) \alpha(N) \\
\vdots & \vdots & \ddots & \vdots \\
\phi_{N / 2}\left(\boldsymbol{r}_{1}\right) \beta(1) & \phi_{N / 2}\left(\boldsymbol{r}_{2}\right) \beta(2) & \ldots & \phi_{N / 2}\left(\boldsymbol{r}_{N}\right) \alpha(N)
\end{array}\right| .
$$

onde $\phi_{i}\left(\boldsymbol{r}_{j}\right)$ corresponde ao $i$-ésimo orbital normalizado do $j$-ésimo elétron, $\alpha(j)$ a projeção positiva de spin do $j$-ésimo elétron $(\uparrow)$ e $\beta(j)$ a projeção negativa $(\downarrow)$. O produto do orbital pela projeção de spin compõe os spinorbitais, $\psi_{i}(\boldsymbol{r})=\phi_{i}(\boldsymbol{r}) \sigma(i)$, que são autoestados globais de um elétron ${ }^{1}$. A formulação determinantal automaticamente garante que o postulado de antissimetrização seja satisfeito e corresponde a solução exata para o problema de $N$ elétrons não interagentes, cujo modelo de Thomas-Fermi é baseado. A densidade eletrônica por sua vez, assume a forma,

$$
\rho(\boldsymbol{r})=\sum_{j=1}^{N}\left|\psi_{j}(\boldsymbol{r})\right|^{2} .
$$

Ao decompor o funcional de energia de maneira conveniente, partindo de (2.13) para o caso de uma densidade genérica, e tendo em vista (2.9), segue que,

$$
\begin{aligned}
E[\rho] & =F[\rho]+\int d \boldsymbol{r} \rho(\boldsymbol{r}) v(\boldsymbol{r}), \\
& =T[\rho]+W[\rho]+\int d \boldsymbol{r} \rho(\boldsymbol{r}) v(\boldsymbol{r}), \\
& \equiv T_{s}[\rho]+J[\rho]+E_{x c}[\rho]+\int d \boldsymbol{r} \rho(\boldsymbol{r}) v(\boldsymbol{r}) .
\end{aligned}
$$

O termo $E_{x c}[\rho]$ é chamado energia de correlação e troca e é dado por,

\footnotetext{
${ }^{1}$ Na notação de spin-orbitais, o vetor de posição no lado esquerdo da identidade passa a incorporar as projeções de spin, sendo definido como $\boldsymbol{r} \equiv(x, y, z, \sigma)$.
} 


$$
E_{x c}[\rho] \equiv T[\rho]-T_{s}[\rho]+W[\rho]-J[\rho] .
$$

Ele contém toda a informação desconhecida do sistema, a diferença entre a energia cinética real e a do gás de elétrons livres, assim como todos os efeitos não clássicos contidos na interação eletrônica. Tendo em mãos algum modelo para $E_{x c}$, o método variacional atrelado à condição de ortogonalidade $^{2}$,

$$
\int d \boldsymbol{r} \psi_{i}^{*}(\boldsymbol{r}) \psi_{j}(\boldsymbol{r})=\delta_{i j}
$$

estabelece um procedimento auto-consistente para obtenção dos spin-orbitais de um elétron,

$$
\delta\left\{E[\rho]-\sum_{i, j=1}^{N} \epsilon_{i j}\left(\int d \boldsymbol{r} \psi_{i}^{*}(\boldsymbol{r}) \psi_{j}(\boldsymbol{r})-\delta_{i j}\right)\right\}=0,
$$

onde $\epsilon_{i j}$ são os multiplicadores de Lagrange associados ao vínculo (2.18). Substituindo (2.16) na expressão acima, obtemos as equações de KohnSham propriamente ditas (ver Apêndice B),

$$
\begin{array}{r}
{\left[-\frac{\nabla^{2}}{2}+v(\boldsymbol{r})+\int \frac{\rho\left(\boldsymbol{r}^{\prime}\right)}{\left\|\boldsymbol{r}-\boldsymbol{r}^{\prime}\right\|} d \boldsymbol{r}^{\prime}+v_{x c}(\boldsymbol{r})\right] \psi_{i}(\boldsymbol{r})=\epsilon_{i} \psi_{i}(\boldsymbol{r})} \\
{\left[-\frac{\nabla^{2}}{2}+v^{K S}\right] \psi_{i}(\boldsymbol{r})=\epsilon_{i} \psi_{i}(\boldsymbol{r})} \\
\hat{h}^{K S} \psi_{i}(\boldsymbol{r})=\epsilon_{i} \psi_{i}(\boldsymbol{r})
\end{array}
$$

onde,

$$
v_{x c}(\boldsymbol{r})=\frac{\delta E_{x c}[\rho]}{\delta \rho(\boldsymbol{r})},
$$

é o potencial de correlação e troca, $\psi_{i}(\boldsymbol{r})$ são os orbitais de Kohn-Sham e $\epsilon_{i}$ suas respectivas energias. Ao computar $\rho(\boldsymbol{r})$ utilizando as autofunções de Kohn-Sham via equação (2.15), a energia total do sistema, dada por (2.16), pode ser reescrita como,

\footnotetext{
${ }^{2}$ Como a notação do vetor de posição incorpora as projeções de spin, $\boldsymbol{r} \equiv(\boldsymbol{r}, \sigma)$, a condição ortogonalidade diz respeito ao espaço de momento angular e ao de spin.
} 


$$
E[\rho(\boldsymbol{r})]=\sum_{i=1}^{N} \epsilon_{i}-\frac{1}{2} \iint d \boldsymbol{r} d \boldsymbol{r}^{\prime} \frac{\rho(\boldsymbol{r}) \rho\left(\boldsymbol{r}^{\prime}\right)}{\left\|\boldsymbol{r}-\boldsymbol{r}^{\prime}\right\|}+E_{x c}[\rho(\boldsymbol{r})]-\int d \boldsymbol{r} v_{x c}(\boldsymbol{r}) \rho(\boldsymbol{r})
$$

Assim sendo, a precisão dos cálculos depende essencialmente do quão bem descrito $E_{x c}$, e consequentemente $v_{x c}$, são, motivando uma nova área de pesquisa dedicada a formulação de funcionais que resulta em um leque de possibilidades adequadas para conjuntos distintos de sistemas e propriedades [46]. Na próxima seção será apresentado o funcional utilizado no presente trabalho.

\subsubsection{O funcional de correlação e troca $\omega \mathrm{B} 97 \mathrm{X}-\mathrm{D}$}

Como ponto de partida para a elaboração dos funcionais de correlação e troca são estabelecidas algumas propriedades exatas. Entre elas as relações de escala, condições de auto-interação, comportamento nos limites assintóticos e descontinuidades das derivadas são responsáveis por manter a realidade física do problema [31]. Desta forma, os funcionais são categorizados de acordo com o nível de aproximação empregado. A figura abaixo corresponde a uma adaptação da Escada de Jacob [48] e ilustra a ascensão em direção à precisão computacional. 


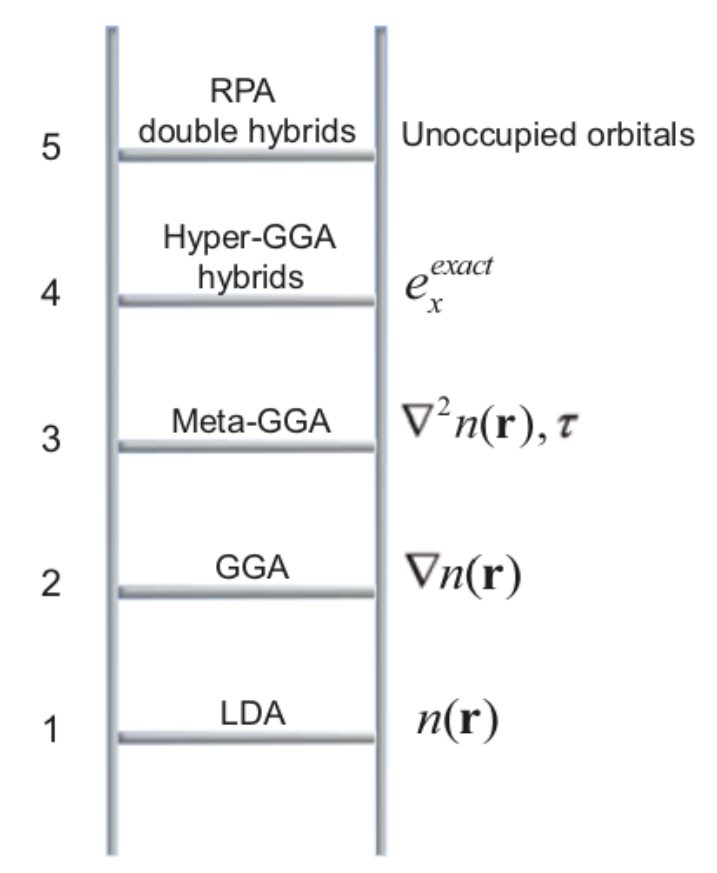

Figura 2.1: Escada de aproximações, adaptada da referência [34], que parte da teoria de Hartree e ilustra o rumo em direção ao ganho de precisão introduzindo as diferentes classes de funcionais. Cada degrau inclui novos elementos na formulação dos mesmos.

Simplificadamente, o primeiro degrau faz referência, por meio da sigla LDA (Local Density Approximation), à proposta introduzida em 1965 por Kohn e Sham, que utiliza do modelo de Thomas-Fermi do gás de elétrons livres (apresentado na Subseção 2.2) para calcular a energia de troca, enquanto a energia de correlação é determinada a partir de uma interpolação de energias, obtidas via Método de Monte Carlo, para diferentes densidades eletrônicas [30, 49]. Os degraus 2 e 3 dispõem de derivadas de primeira e segunda ordem na densidade eletrônica, enquanto os últimos degraus, além de envolverem parametrizações a partir de dados experimentais, adicionam a contribuição exata do termo de troca e eventuais refinamentos através da construção de uma teoria que envolva estados excitados.

O funcional utilizado nos cálculos DFT e TDDFT do presente trabalho reside no quarto degrau e foi formulado pelos pesquisadores Jeng-Da Chai e Martin Head-Gordon em 2008 [50, 51]. Batizado de $\omega$ B97X-D, o funcional em questão é híbrido e possui correção de longo alcance, que por meio da decomposição,

$$
\frac{1}{r_{12}}=\frac{\operatorname{erf}\left(\omega r_{12}\right)}{r_{12}}+\frac{\operatorname{erfc}\left(\omega r_{12}\right)}{r_{12}}
$$


onde $r_{12} \equiv\left\|\boldsymbol{r}_{1}-\boldsymbol{r}_{2}\right\|$, erf é a função erro e erfc a função erro complementar, retoma o termo HF de troca no limite assintótico $\left(r_{12} \rightarrow \infty\right)$, o que reduz significativamente o erro de auto-interação presente em funcionais híbridos globais, como B97 e B3LYP. O funcional pode ser decomposto em cinco partes,

$$
E_{x c}^{\omega \mathrm{B} 97 \mathrm{X}-\mathrm{D}}=E_{x}^{\mathrm{LR}-\mathrm{HF}}+c_{x} E_{x}^{\mathrm{SR}-\mathrm{HF}}+E_{x}^{\mathrm{SR}-\mathrm{B} 97}+E_{c}^{\mathrm{B} 97}+E_{\text {disp }} .
$$

Os dois primeiros termos remetem à correção introduzida acima, explicitamente dados por,

$$
E_{x}^{\mathrm{LR}-\mathrm{HF}}=-\frac{1}{2} \sum_{\sigma} \sum_{i, j}^{o c .} \iint d \boldsymbol{r}_{1} d \boldsymbol{r}_{2} \psi_{i \sigma}^{*}\left(\boldsymbol{r}_{1}\right) \psi_{j \sigma}^{*}\left(\boldsymbol{r}_{1}\right) \frac{\operatorname{erf}\left(\omega r_{12}\right)}{r_{12}} \psi_{i \sigma}\left(\boldsymbol{r}_{2}\right) \psi_{j \sigma}\left(\boldsymbol{r}_{2}\right),
$$

e

$$
E_{x}^{\mathrm{SR}-\mathrm{HF}}=-\frac{1}{2} \sum_{\sigma} \sum_{i, j}^{o c .} \iint d \boldsymbol{r}_{1} d \boldsymbol{r}_{2} \psi_{i \sigma}^{*}\left(\boldsymbol{r}_{1}\right) \psi_{j \sigma}^{*}\left(\boldsymbol{r}_{1}\right) \frac{\operatorname{erfc}\left(\omega r_{12}\right)}{r_{12}} \psi_{i \sigma}\left(\boldsymbol{r}_{2}\right) \psi_{j \sigma}\left(\boldsymbol{r}_{2}\right),
$$

sendo $\psi_{i, \sigma}(\boldsymbol{r})$ os spin-orbitais e as somas sobre todas as projeções de spin $\sigma$ e sobre todos os estados ocupados. O parâmetro $c_{x}$ é uma constante ajustada de forma a garantir o comportamento assintótico $1 / r$. Os dois termos seguintes remetem aos trabalhos de Becke [52], sendo $E_{x}^{\mathrm{SR}-\mathrm{B} 97}$ sujeito a uma correção adicional de curto alcance por meio de uma função de atenuação e o $E_{c}^{\mathrm{B} 97}$ o termo de correlação propriamente introduzido em 1997 (ver Apêndice C). Não menos importante, é contabilizada uma correção de dispersão na energia, dada por,

$$
E_{\text {disp }}=-\sum_{i=1}^{N_{a t}-1} \sum_{j=i+1}^{N_{a t}} \frac{C_{6}^{i j}}{R_{i j}^{6}} f_{d a m p}\left(R_{i j}\right)
$$

sendo,

$$
f_{\text {damp }}\left(R_{i j}\right)=\frac{1}{1+a\left(\frac{R_{i j}}{R_{r}}\right)^{-12}}
$$

onde $N_{a t}$ é o número de átomos do sistema, $C_{6}^{i j}$ o coeficiente de dispersão do par atômico $i j$ e $R_{i j}$ a respectiva distância interatômica. $a$ corresponde ao único parâmetro não-linear que atua como raio de corte e é ajustado 
de forma a controlar a intensidade da energia de dispersão, e $R_{r}$ a soma dos raios de van-der-Waals do $i$-ésimo e do $j$-ésimo átomo [53]. Tal aperfeiçoamento busca resgatar parte da correlação eletrônica de longo alcance responsável pelas interações intermoleculares, sendo essas essenciais para a descrição adequada de diversos sistemas quânticos. A escolha desse funcional foi motivada pelo uso do mesmo na literatura para caracterizar doadores e aceitadores eletrônicos $[54,55,21,22,56,57]$.

\subsubsection{A base atômica $6-31 \mathrm{G}(\mathrm{d}, \mathrm{p})$}

Em decorrência do interesse em validar a metodologia DFTB, para os cálculos DFT foi utilizada a base atômica 6-31G(d,p) (também chamada de $\left.6-31 G^{* *}\right)$ de tamanho modesto que, em vista do custo computacional, é frequentemente usada na literatura para estudar sistemas grandes (como interfaces D:A) e processos de transferência de carga $[21,55,58]$.

A base em questão é descrita na notação de Pople ou split-valence que foi introduzida por John A. Pople e colaboradores [59], e utiliza de funções do tipo gaussianas para expandir os orbitais atômicos. Como exemplo, as gaussianas normalizadas do tipo $1 s, 2 p_{y}$ e $3 d_{y z}$, são,

$$
\begin{aligned}
& g_{1 s}(\zeta, \boldsymbol{r})=\left(\frac{8 \zeta^{3}}{\pi^{3}}\right)^{\frac{1}{4}} e^{-\zeta \boldsymbol{r}^{2}} \\
& g_{2 p_{y}}(\zeta, \boldsymbol{r})=\left(\frac{128 \zeta^{5}}{\pi^{3}}\right)^{\frac{1}{4}} y e^{-\zeta \boldsymbol{r}^{2}}, \\
& g_{3 d_{y z}}(\zeta, \boldsymbol{r})=\left(\frac{2048 \zeta^{7}}{\pi^{3}}\right)^{\frac{1}{4}} y z e^{-\zeta \boldsymbol{r}^{2}} .
\end{aligned}
$$

O uso de gaussianas está diretamente atrelado ao ganho computacional durante cálculos de estrutura eletrônica, principalmente em decorrência dos frequentes cálculos de integrais de quatro centros, da forma,

$$
\left(n_{A} m_{B}\left|f\left(\boldsymbol{r}_{1}, \boldsymbol{r}_{2}\right)\right| l_{C} k_{D}\right)=\int d \boldsymbol{r}_{1} d \boldsymbol{r}_{2} \psi_{n, A}^{*}\left(\boldsymbol{r}_{1}\right) \psi_{m, B}^{*}\left(\boldsymbol{r}_{1}\right) f\left(\boldsymbol{r}_{1}, \boldsymbol{r}_{2}\right) \psi_{l, C}\left(\boldsymbol{r}_{2}\right) \psi_{k, D}\left(\boldsymbol{r}_{2}\right),
$$

onde $\psi_{i, I}\left(\boldsymbol{r}_{1}\right)$ é uma função de base no núcleo $I$ (centrada em $\boldsymbol{R}_{I}$ ) referente ao $i$-ésimo estado, e $f\left(\boldsymbol{r}_{1}, \boldsymbol{r}_{2}\right)$ é o kernel. O produto de duas funções gaussianas $g(\zeta, \boldsymbol{r})$ é, a menos de uma constante, uma gaussiana ${ }^{3}$,

\footnotetext{
${ }^{3}$ Os parâmetros $K_{A B}, \zeta_{3}$ e $\boldsymbol{R}_{A B}$ são obtidos diretamente das variáveis iniciais $\zeta_{1}, \zeta_{2}, \boldsymbol{R}_{A}$ e $\boldsymbol{R}_{B}$, como indicado em [60].
} 


$$
g\left(\zeta_{1}, \boldsymbol{r}-\boldsymbol{R}_{A}\right) g\left(\zeta_{2}, \boldsymbol{r}-\boldsymbol{R}_{B}\right)=K_{A B} g\left(\zeta_{3}, \boldsymbol{r}-\boldsymbol{R}_{A B}\right),
$$

o que simplifica o cálculo de (2.26), viabilizando o aumento no número de funções de base. Assim sendo, a base escolhida dispõe de 6 funções gaussianas normalizadas para descrever os orbitais de caroço, 4 para os orbitais de valência e dois conjuntos de funções de polarização, uma do tipo $p$ e outro do tipo $d$. Por exemplo, a descrição do caroço de um átomo de carbono é realizada por meio da contração das 6 gaussianas normalizadas,

$$
\psi_{1 s}(\boldsymbol{r})=\sum_{i=1}^{6} d_{i, 1 s} g_{1 s}\left(\zeta_{i, 1 s}, \boldsymbol{r}\right),
$$

na qual os orbitais atômicos $\left(\psi_{x}\right)$ são obtidos a partir de combinações lineares das mesmas $\left(g_{x}\right)$, com coeficientes $\left(d_{i, x}\right)$ e parâmetros $\left(\zeta_{i, x}\right)$ otimizados, usualmente, de forma a minimizar a energia total do sistema. De maneira análoga, para os orbitais de valência é realizada a contração de 4 funções gaussianas normalizadas com seus respectivos coeficientes otimizados. A seguir, são construídos dois orbitais atômicos associados a um mesmo momento angular, o orbital exterior que corresponde a função mais difusa (menor expoente) e o interior que é dado pela contração das outras três. Considerando por exemplo a faixa Li-Ne (na ausência de funções de polarização), ${ }^{4}$

$$
\begin{aligned}
& \psi_{2 s}^{\text {out }}(\boldsymbol{r})=g_{1 s}\left(\zeta_{2 s p}^{\prime}, \boldsymbol{r}\right) \\
& \psi_{2 s}^{\text {in }}(\boldsymbol{r})=\sum_{i=1}^{3} d_{i, 2 s} g_{1 s}\left(\zeta_{2 s p}^{\prime \prime}, \boldsymbol{r}\right)
\end{aligned}
$$

Esse procedimento garante maior flexibilidade para a descrição do problema e promove a base à categoria duplo zeta. A importância das funções de polarização pode ser entendida ao considerar o efeito da molécula sobre cada átomo. É de se esperar que os orbitais atômicos não se comportem exatamente como no vácuo, o que pode ser entendido como uma espécie de campo elétrico médio induzindo uma polarização na molécula. Neste contexto entram os conjuntos adicionais de polarização, 3 funções gaussianas do tipo $p$ para os átomos de hidrogênio e 5 do tipo $d$ para os átomos na faixa Li-Ne. ${ }^{5}$ Além de reproduzir em cada átomo os efeitos do meio mate-

\footnotetext{
${ }^{4} \mathrm{~A}$ notação $\zeta_{2 s p}$ ilustra que os coeficientes das gaussianas do tipo $2 s$ e $2 p$ são iguais, a fim de reduzir o custo computacional.

${ }^{5} \mathrm{Na}$ prática também é adicionada uma função a mais do tipo $3 s\left(x^{2}+y^{2}+z^{2}\right)$ por uma questão de conveniência computacional.
} 
rial (polarização), o aumento do número de funções base possibilita melhor caracterização do ponto de vista variacional, aprimorando a descrição do problema.

\subsection{Teoria do Funcional de Densidade Dependente do Tempo}

Ainda que o estudo de compostos no estado fundamental seja de grande importância, para poder descrever estados excitados é necessário recorrer a vertente dependente do tempo da DFT. Embasadas pelos teoremas de Runge-Gross e van Leeuwen ${ }^{6}$ que dentro de certas restrições estabelecem o mapeamento unívoco entre observáveis e a densidade eletrônica, algumas propostas como o formalismo das equações Kohn-Sham acopladas (CKS), método da Função de Green de Stott e Zaremba e o método de Sternheimer adaptado por Mahan se distanciaram do estado fundamental por meio da caracterização do efeito de potenciais estáticos sobre sistemas de alta simetria [62]. Entretanto, apenas em 1995, através da Teoria de Resposta Linear, Mark E. Casida formalizou de maneira prática um conjunto de equações matriciais que, por meio do tratamento de pequenas perturbações dependentes do tempo [62], possibilitam a determinação de estados excitados e observáveis.

Tal formalismo foi batizado de LR-TDDFT (Linear Response Time-Dependent Density Functional Theory) e devido a sua popularização frente as outras abordagens, hoje é comumente chamado apenas de TDDFT (TimeDependent Density Functional Theory).

\subsubsection{Teorema de Runge-Gross}

De maneira análoga ao que foi realizado na Subseção 2.2.1, buscamos estabelecer uma relação unívoca entre o potencial externo e a densidade eletrônica, ambos dependentes do tempo, de tal forma que o potencial possa ser descrito como um funcional da densidade e vice-versa. O primeiro teorema fundamental foi proposto por Runge e Gross em 1984 e pode ser entendido de acordo com o texto abaixo.

\footnotetext{
${ }^{6} \mathrm{Na}$ descrição relativística da TDDFT o teorema de Rajagopal-Callaway [61] corresponde a generalização do teorema de Hohenberg-Kohn.
} 
Teorema de Runge-Gross: Considere duas densidades eletrônicas $\rho(\boldsymbol{r}, t)$ e $\rho^{\prime}(\boldsymbol{r}, t)$ que partem do mesmo estado inicial global $\Psi_{0}$, sujeitas à ação de dois potenciais diferentes, $v(\boldsymbol{r}, t)$ e $v^{\prime}(\mathbf{r}, t) \neq v(\boldsymbol{r}, t)+c(t)$, onde $c(t)$ é constante com relação as coordenadas espaciais. Admitindo que a dependência temporal é dada para $t>t_{0}$, existe uma correspondência única entre cada potencial externo e a densidade do estado fundamental correspondente.

A demonstração desse teorema contêm diversas sutilezas [63]. No caso independente do tempo, a correspondência entre função de onda $\Psi$ e densidade eletrônica $\rho(\boldsymbol{r}, t)$ é unívoca a menos de uma fase arbitrária $e^{i c}$. Já no caso dependente do tempo, as funções de onda são dadas a menos de uma fase também depende do tempo $e^{i c(t)}$, de tal forma que a correspondência biunívoca entre $\Psi$ e $\rho(\boldsymbol{r}, t)$ é válida apenas para uma fase específica. Tal complicação pode ser contornada expressando a função de onda como funcional do potencial externo $\Psi[v(\boldsymbol{r}, t)]$, o que estabelece a correspondência unívoca com a densidade eletrônica mas além de perder o conceito do funcional global $F[\rho]$, está sujeita as descontinuidades nas derivadas de $\Psi$ e $v(\boldsymbol{r}, t)$.

Uma alternativa para esse problema, detalhada por Ullrich [34], é admitir que o potencial externo é uma função analítica no tempo e portanto pode ser expresso por uma série de Taylor em torno do instante $t_{0}$, no qual a perturbação é iniciada. Entretanto, Yang e Burke mostraram que tais potenciais não existem fisicamente [64], sendo necessário recorrer ao formalismo da Eletrodinâmica Quântica para contornar tais problemas, como foi realizado por Rajagopal e Callaway [61].

\subsubsection{Teorema de van Leeuwen}

Uma vez convencidos do mapeamento entre potencial externo e densidade eletrônica, o próximo passo consiste em buscar sistemas não-interagentes que reproduzam as mesmas densidades do sistemas reais, de maneira análoga ao caso estacionário. O teorema que proporciona tais soluções foi primeiro demonstrado por Robert van Leeuwen em seus trabalhos publicados a partir de 1998 [65, 66, 67]. 
Teorema de van Leeuwen: Considere a densidade eletrônica $\rho(\boldsymbol{r}, t)$ que representa um sistema de muitos corpos com uma dada interação entre partículas $w\left(\left\|\boldsymbol{r}-\boldsymbol{r}^{\prime}\right\|\right)$, potencial externo $v(\boldsymbol{r}, t)$ e estado inicial $\Psi_{0}$. Para outra interação entre partículas $w^{\prime}\left(\left\|\boldsymbol{r}-\boldsymbol{r}^{\prime}\right\|\right)$, existe um único potencial externo $v^{\prime}(\boldsymbol{r}, t)$ (a menos de uma constante exclusivamente dependente do tempo, $c(t))$ que reproduz a mesma densidade eletrônica dependente do tempo $n(\boldsymbol{r}, t)$. O estado inicial $\Psi_{0}^{\prime}$ deve ser escolhido de tal forma que reproduza a densidade correta.

Ainda que o resultado seja geral, é possível escolher o caso trivial de partículas não interagentes no qual a interação é nula, $w^{\prime}=0$, e o teorema garante o mapeamento um-pra-um entre o novo potencial externo $v^{\prime}(\boldsymbol{r}, t)$ e a densidade eletrônica do sistema real, $n(\boldsymbol{r}, t)$, possibilitando a construção das equações de KS no formalismo dependente do tempo.

A aplicabilidade do teorema permanece um problema em aberto, uma vez que a demonstração do caso geral envolve a expansão em séries de Taylor dos potenciais externos. É necessário que a densidade eletrônica seja analítica no tempo, um fator limitante no estudo da evolução temporal do sistema, uma vez que átomos, moléculas e sólidos na aproximação de Born-Oppenheimer possuem descontinuidades nas posições dos núcleos [64]. Tais questionamentos caracterizam uma área de pesquisa em torno da v-representabilidade da densidade eletrônica, que busca responder de maneira geral, quando podemos garantir a existência de um potencial que reproduza uma determinada densidade eletrônica. A demonstração do teorema de van Leeuwen envolvendo a expansão em polinômios de Taylor pode ser encontrada na referência [66] e outras tentativas na ausência dessa hipótese nas referências [68, 69].

\subsubsection{Teoria de Resposta Linear}

A Teoria de Resposta Linear é um método utilizado em diversos segmentos da Física quando é de interesse entender a resposta do sistema diante de uma perturbação fraca. Matematicamente, o valor esperado de uma determinada grandeza é expandido em uma série de Volterra truncada no termo de primeira ordem. Essas séries se destacam pois levam em consideração efeitos de memória, ou seja, a resposta depende do compor- 
tamento da perturbação ao longo de todos os instantes de tempo ${ }^{7}$, sendo frequentemente utilizadas na modelagem de problemas não lineares. Antes de entrar no formalismo é importante caracterizar o operador unitário de evolução temporal na representação de interação. Considere a equação de Schrödinger dependente do tempo em unidades atômicas,

$$
\hat{\mathcal{H}}(t) \Psi\left(\boldsymbol{x}_{1}, \ldots, \boldsymbol{x}_{N}, t\right)=i \frac{\partial}{\partial t} \Psi\left(\boldsymbol{x}_{1}, \ldots, \boldsymbol{x}_{N}, t\right)
$$

onde $\boldsymbol{x}_{i}$ é coordenada do $i$-ésimo elétron e $\hat{\mathcal{H}}(t)$ o operador hamiltoniano dependente do tempo. Uma solução formal de (2.27) é,

$$
\Psi(t)=\hat{U}\left(t, t_{0}\right) \Psi_{0},
$$

tal que $\hat{U}\left(t, t_{0}\right)$ é o operador de evolução temporal que age sobre o estado inicial $\Psi_{0}$ resultando em $\Psi(t)$ para $t \geq t_{0}$. No caso em que a hamiltoniana pode ser decomposta em duas partes,

$$
\hat{\mathcal{H}}(t)=\hat{\mathcal{H}}_{0}+\hat{\mathcal{H}}_{1}(t),
$$

onde $\hat{\mathcal{H}}_{0}$ é a hamiltoniana cujos autoestados e autofunções são bem definidos e $\hat{\mathcal{H}}_{1}(t)$ descreve a perturbação dependente do tempo que é finita para $t \geq t_{0} \cdot{ }^{8}$ É conveniente definir o operador de evolução temporal como,

$$
\hat{U}\left(t, t_{0}\right)=e^{-i \hat{\mathcal{H}}_{0}\left(t-t_{0}\right)} \hat{U}_{1}\left(t, t_{0}\right) .
$$

Substituindo (2.28) em (2.27) e considerando apenas a solução de primeira ordem (ver Apêndice $\mathrm{D}$ ), o operador $\hat{U}\left(t, t_{0}\right)$ é dado por,

$$
\hat{U}\left(t, t_{0}\right)=e^{-i \hat{\mathcal{H}}_{0}\left(t-t_{0}\right)}\left\{1-i \int_{t_{0}}^{t} d t^{\prime} e^{i \hat{\mathcal{H}}_{0}\left(t^{\prime}-t_{0}\right)} \hat{\mathcal{H}}_{1}\left(t^{\prime}\right) e^{-i \hat{\mathcal{H}}_{0}\left(t^{\prime}-t_{0}\right)}\right\} .
$$

Assumindo que a perturbação possa ser expressa como,

$$
\hat{\mathcal{H}}_{1}(t)=F(t) \hat{\mathcal{O}}
$$

onde $F(t)$ é um campo externo e $\hat{\mathcal{O}}$ um operador, que na representação de

\footnotetext{
${ }^{7}$ Em oposição, séries de Taylor levam em consideração apenas a perturbação em um instante de tempo fixo.

${ }^{8}$ Essa decomposição pode ser exemplificada por um sistema que se encontra no estado fundamental e num dado instante de tempo $t=t_{0}$ é submetido a uma perturbação externa, e.g. o campo elétrico de um laser monocromático.
} 
interação é dado por,

$$
\hat{\mathcal{O}}(t)=e^{i \hat{\mathcal{H}}_{0} t} \hat{\mathcal{O}} e^{-i \hat{\mathcal{H}}_{0} t}
$$

Comparando (2.31) com a integral da equação (2.29), podemos identificar a evolução de $\hat{\mathcal{O}}$ e portanto o operador de evolução temporal assume a forma,

$$
\hat{U}\left(t, t_{0}\right)=e^{-i \hat{\mathcal{H}}_{0}\left(t-t_{0}\right)}\left\{1-i \int_{t_{0}}^{t} d t^{\prime} F\left(t^{\prime}\right) \hat{\mathcal{O}}\left(t^{\prime}-t_{0}\right)\right\} .
$$

Considere um observável $\hat{\alpha}$, tal que seu valor esperado no estado fundamental seja,

$$
\alpha_{0}=\left\langle\Psi_{0}|\hat{\alpha}| \Psi_{0}\right\rangle,
$$

onde $\Psi_{0}$ é a função de onda do estado fundamental da hamiltoniana estática $\hat{\mathcal{H}}_{0}$. No caso em que o sistema é sujeito a uma perturbação externa da forma (2.30), o valor do observável em um dado instante de tempo $t \geq t_{0}$ é dado por,

$$
\alpha(t)=\langle\Psi(t)|\hat{\alpha}| \Psi(t)\rangle .
$$

A diferença entre $\alpha(t)$ e o valor estático $\alpha_{0}$ leva o nome de resposta do observável frente à perturbação $\hat{\mathcal{H}}_{1}(t)$, e pode ser expressa a partir de uma em série de Taylor que parte da primeira ordem,

$$
\alpha(t)-\alpha_{0}=\alpha_{1}(t)+\alpha_{2}(t)+\alpha_{3}(t)+\ldots
$$

onde $\alpha_{1}(t)$ é o termo de resposta linear, $\alpha_{2}(t)$ o termo quadrático e assim sucessivamente. Utilizando a representação de interação (2.31) para ambos os operadores $\hat{\mathcal{O}}(t)$ e $\hat{\alpha}(t)$, assim como a aproximação de primeira ordem do operador de evolução temporal (2.32), a resposta linear assume a forma (ver Apêndice E),

$$
\alpha_{1}(t)=\int_{-\infty}^{\infty} d t^{\prime} \chi_{\alpha \mathcal{O}}\left(t-t^{\prime}\right) F\left(t^{\prime}\right)
$$

onde $\chi_{\alpha \mathcal{O}}\left(t-t^{\prime}\right)$ é a função de resposta retardada, definida como,

$$
\chi_{\alpha \mathcal{O}}\left(t-t^{\prime}\right)=-i \theta\left(t-t^{\prime}\right)\left\langle\Psi_{0}\left|\left[\hat{\alpha}\left(t-t^{\prime}\right), \hat{\mathcal{O}}\right]\right| \Psi_{0}\right\rangle .
$$

A nomenclatura retardada é consequência da resposta no instante $t$ ser 
devido a perturbação em um instante anterior $t^{\prime} \leq t$, e tem a causalidade preservada pela utilização da função de Heaviside,

$$
\theta\left(t-t^{\prime}\right)=\left\{\begin{array}{l}
0, \quad t<t^{\prime} \\
\frac{1}{2}, \quad t=t^{\prime} \\
1, \quad t>t^{\prime}
\end{array} .\right.
$$

No que diz respeito à determinação das energias de excitação, é conveniente trabalhar no espaço das frequências. Seja $\tilde{F}(\omega)$ a transformada de Fourier da perturbação $F(t)$, logo,

$$
F(t)=\frac{1}{2 \pi} \int_{-\infty}^{\infty} d \omega \tilde{F}(\omega) e^{-i \omega t} \text { e } \tilde{F}(\omega)=\int_{-\infty}^{\infty} d t F(t) e^{i \omega t}
$$

As demais variáveis dependentes do tempo como $\alpha(t)$ e $\chi_{\alpha \mathcal{O}}$ também se transformam de maneira análoga. Partindo da expressão (2.35) para a resposta linear,

$$
\begin{aligned}
& \alpha_{1}(t)=\int_{-\infty}^{\infty} d t^{\prime} \chi_{\alpha \mathcal{O}}\left(t-t^{\prime}\right) F\left(t^{\prime}\right) \\
& \frac{1}{2 \pi} \int_{-\infty}^{\infty} d \omega \tilde{\alpha_{1}}(\omega) e^{-i \omega t}=\int_{-\infty}^{\infty} d t^{\prime}\left(\frac{1}{2 \pi} \int_{-\infty}^{\infty} d \omega \chi_{\alpha} \mathcal{O}(\omega) e^{-i \omega\left(t-t^{\prime}\right)}\right)\left(\frac{1}{2 \pi} \int_{-\infty}^{\infty} d \omega^{\prime} \tilde{F}\left(\omega^{\prime}\right) e^{-i \omega^{\prime} t^{\prime}}\right) \\
& \int_{-\infty}^{\infty} d \omega \tilde{\alpha_{1}}(\omega) e^{-i \omega t}=\frac{1}{2 \pi} \int_{-\infty}^{\infty} d \omega \int_{-\infty}^{\infty} d \omega^{\prime} \underbrace{\left(\int_{-\infty}^{\infty} d t^{\prime} e^{i t\left(\omega-\omega^{\prime}\right)}\right)}_{2 \pi \delta\left(\omega-\omega^{\prime}\right)} \chi_{\tilde{\alpha} \mathcal{O}}(\omega) e^{-i \omega t} \tilde{F}\left(\omega^{\prime}\right) .
\end{aligned}
$$

Com auxílio da relação de ortogonalidade das funções exponenciais a função delta é introduzida reduzindo as integrações para a variável $\omega$,

$$
\begin{aligned}
& \int_{-\infty}^{\infty} \tilde{\alpha}_{1}(\omega) e^{-i \omega t}=\int_{-\infty}^{\infty} d \omega \tilde{\chi}_{\alpha \mathcal{O}}(\omega) e^{-i \omega t} \tilde{F}(\omega) \\
& \int_{-\infty}^{\infty} d \omega e^{-i \omega t}\left\{\tilde{\alpha}_{1}(\omega)-\tilde{\chi}_{\alpha \mathcal{O}}(\omega) \tilde{F}(\omega)\right\}=0 .
\end{aligned}
$$

Como as exponenciais $e^{-i \omega t}$ são linearmente independentes, obtemos uma equação para a resposta linear no espaço de frequências,

$$
\tilde{\alpha}_{1}(\omega)=\tilde{\chi}_{\alpha \mathcal{O}}(\omega) \tilde{F}(\omega) .
$$

Redirecionando a atenção para a função de resposta linear no espaço de frequências, pela equação $(2.36)$, 


$$
\tilde{\chi}_{\alpha \mathcal{O}}(\omega)=-i \int_{-\infty}^{\infty} d \tau \theta(\tau)\left\langle\Psi_{0}|[\hat{\alpha}(\tau), \hat{\mathcal{O}}]| \Psi_{0}\right\rangle e^{i \omega \tau}
$$

onde $\tau \equiv t-t^{\prime}$. Introduzindo o conjunto completo de autofunções da hamiltoniana $H_{0}$ e utilizando da representação integral da função de Heaviside, obtemos a representação de Lehmann da função de resposta linear (ver Apêndice F),

$$
\tilde{\chi}_{\alpha \mathcal{O}}(\omega)=\lim _{\eta \rightarrow 0+} \sum_{n=1}^{\infty}\left\{\frac{\left\langle\Psi_{0}|\hat{\alpha}| \Psi_{n}\right\rangle\left\langle\Psi_{n}|\hat{\mathcal{O}}| \Psi_{0}\right\rangle}{\omega-\Omega_{n}+i \eta}-\frac{\left\langle\Psi_{0}|\hat{\mathcal{O}}| \Psi_{n}\right\rangle\left\langle\Psi_{n}|\hat{\alpha}| \Psi_{0}\right\rangle}{\omega+\Omega_{n}+i \eta}\right\}
$$

onde $\Omega_{n} \equiv E_{n}-E_{0}$ é a $n$-ésima energia de excitação. Esse resultado é de grande importância pois mostra como uma perturbação dependente da frequência se acopla com o espectro de estados excitados de um dado sistema. Os polos de $\tilde{\chi}_{\alpha \mathcal{O}}(\omega)$ correspondem as energias de excitação $\Omega_{n}$.

\subsubsection{Resposta linear densidade-densidade}

A função resposta de interesse é a da densidade eletrônica frente a uma perturbação que acopla com o próprio operador de densidade, o que possibilita a elaboração de um procedimento auto-consistente para determinar as energias de excitação. Considere o potencial externo dependente do tempo da forma,

$$
v(\boldsymbol{r}, t)=v_{0}(\boldsymbol{r})+v_{1}(\boldsymbol{r}, t) \theta\left(t-t_{0}\right),
$$

onde $v_{0}(\boldsymbol{r})$ é o potencial externo da hamiltoniana eletrônica não perturbada (2.7) para $t<t_{0}$ e $v_{1}(\boldsymbol{r}, t)$ a perturbação com início em $t=t_{0}$, tal que a hamiltoniana (2.30) acoplada ao operador de densidade é dada por,

$$
\hat{\mathcal{H}}_{1}(t)=\int d^{3} r^{\prime} v_{1}\left(\boldsymbol{r}^{\prime}, t\right) \hat{\rho}\left(\boldsymbol{r}^{\prime}\right) .
$$

De acordo com (2.35), a correção de primeira ordem para a densidade eletrônica é,

$$
\rho_{1}(\boldsymbol{r}, t)=\int_{-\infty}^{\infty} d t^{\prime} \int d^{3} r^{\prime} \chi_{\rho \rho}\left(\boldsymbol{r}, \boldsymbol{r}^{\prime}, t-t^{\prime}\right) v_{1}\left(\boldsymbol{r}^{\prime}, t^{\prime}\right)
$$

onde $\chi_{\rho \rho}$ é a função de resposta linear, que por construção pode ser expressa em termos da derivada funcional da densidade com relação ao potencial 
externo, calculada em torno do potencial inicial, $v_{0}(\boldsymbol{r}),{ }^{9}$

$$
\chi\left(\boldsymbol{r}, \boldsymbol{r}^{\prime}, t-t^{\prime}\right)=\left.\frac{\delta \rho[v](\boldsymbol{r}, t)}{\delta v\left(\boldsymbol{r}^{\prime}, t^{\prime}\right)}\right|_{v_{0}(\boldsymbol{r})} .
$$

A notação enfatiza a relação unívoca entre $\rho$ e $v$ e por simplicidade o índice $\rho \rho$ é omitido, pois iremos lidar apenas com respostas do tipo densidadedensidade. Por depender do conjunto completo de auto-estados, o cálculo de $\chi\left(\boldsymbol{r}, \boldsymbol{r}^{\prime}, t-t^{\prime}\right)$ via (2.38) é inviabilizado uma vez que nem sempre conhecemos os autoestados $\left|\Psi_{n}\right\rangle$ quando $n \neq 0$, e para contornar esse problema nos utilizamos do sistema auxiliar não interagente. No contexto de KohnSham, as equações (2.20) resultam no potencial efetivo da forma,

$$
v_{s}[\rho](\boldsymbol{r}, t) \equiv v^{K S}(\boldsymbol{r}, t)=v(\boldsymbol{r}, t)+\int d^{3} r^{\prime} \frac{\rho\left(\boldsymbol{r}^{\prime}, t\right)}{\left\|\boldsymbol{r}-\boldsymbol{r}^{\prime}\right\|}+v_{x c}[\rho](\boldsymbol{r}, t),
$$

onde o potencial de correlação e troca segue a definição (2.21) considerando o funcional e a densidade dependentes do tempo. Conforme o teorema de Van Leeuwen apresentado na Seção 2.3.2, o vínculo entre os sistemas real e não interagente garante que as densidades oriundas dos dois casos sejam iguais, e portanto podemos estabelecer a conexão entre as funções de resposta de cada sistema,

$$
\begin{aligned}
\chi\left(\boldsymbol{r}, \boldsymbol{r}^{\prime}, t-t^{\prime}\right)= & \chi_{s}\left(\boldsymbol{r}, \boldsymbol{r}^{\prime}, t-t^{\prime}\right)+\int d \tilde{t} \int d^{3} x \int d \tilde{t}^{\prime} \int d^{3} x^{\prime}\left\{\frac{\delta\left(\tilde{t}-\tilde{t}^{\prime}\right)}{\left\|\boldsymbol{x}-\boldsymbol{x}^{\prime}\right\|}+\right. \\
& \left.+f_{x c}\left(\boldsymbol{x}, \boldsymbol{x}^{\prime}, \tilde{t}, \tilde{t}^{\prime}\right)\right\} \chi\left(\boldsymbol{x}^{\prime}, \boldsymbol{r}^{\prime}, \tilde{t}^{\prime}-t^{\prime}\right)
\end{aligned}
$$

onde $\chi_{s}$ é a função de resposta linear do sistema de Kohn-Sham cujo kernel é definido como a derivada funcional de $v_{x c}$ com relação a densidade, calculado em torno da densidade do estado fundamental. Ao mudar para o espaço recíproco por meio das transformadas de Fourier, a correção de primeira ordem na densidade pode ser escrita como,

$$
\rho_{1}(\boldsymbol{r}, \omega)=\int d^{3} r^{\prime} \chi_{s}\left(\boldsymbol{r}, \boldsymbol{r}^{\prime}, \omega\right)\left[v_{1}\left(\boldsymbol{r}^{\prime}, \omega\right)+\int d^{3} x\left\{\frac{\delta(\omega)}{\left\|\boldsymbol{r}^{\prime}-\boldsymbol{x}\right\|}+f_{x c}\left(\boldsymbol{r}^{\prime}, \boldsymbol{x}, \omega\right)\right\} \rho_{1}(\boldsymbol{x}, \omega)\right],
$$

\footnotetext{
${ }^{9}$ Essa definição é consequência da expansão de Taylor (2.34) e é conveniente para caracterizar cada função de resposta linear.
} 
onde $\chi_{s}\left(\boldsymbol{r}, \boldsymbol{r}^{\prime}, \omega\right)$ é dada por,

$$
\chi_{s}\left(\boldsymbol{r}, \boldsymbol{r}^{\prime}, \omega\right)=\lim _{\eta \rightarrow 0+} \sum_{k, l}\left(f_{k}-f_{l}\right) \frac{\varphi_{l}(\boldsymbol{r}) \varphi_{k}^{*}(\boldsymbol{r}) \varphi_{k}\left(\boldsymbol{r}^{\prime}\right) \varphi_{l}^{*}\left(\boldsymbol{r}^{\prime}\right)}{\omega-\omega_{l k}+i \eta}
$$

sendo $f_{i}$ o número de ocupação do $i$-ésimo orbital de Kohn-Sham e $\omega_{l k}=$ $\epsilon_{l}-\epsilon_{k}$ a diferença de energia entre os autovalores associados aos orbitais $\psi_{l}$ e $\psi_{k} \cdot{ }^{10}$ As deduções das expressões acima se encontram no Apêndice G. O kernel no espaço de frequências assume a forma,

$$
f_{x c}\left(\boldsymbol{r}, \boldsymbol{r}^{\prime}, \omega\right)=\int d \tau e^{i \omega \tau}\left(\left.\frac{\delta v_{x c}[\rho](\boldsymbol{r}, t)}{\delta \rho\left(\boldsymbol{r}^{\prime}, t^{\prime}\right)}\right|_{n_{0}(\boldsymbol{r})}\right) .
$$

Por fim, é importante incorporar efeitos de spin no formalismo. Como o operador de densidade não atua no espaço de spins, a generalização do método é natural, de tal forma que e a densidade, o potencial linearizado e a função de resposta do sistema não interagente passam a ser, respectivamente,

$$
\begin{gathered}
\rho_{1 \sigma}(\boldsymbol{r}, \omega)=\sum_{\sigma^{\prime}} \int d^{3} r^{\prime} \chi_{s, \sigma \sigma^{\prime}}\left(\boldsymbol{r}, \boldsymbol{r}^{\prime}, \omega\right) v_{s 1, \sigma^{\prime}}\left(\boldsymbol{r}^{\prime}, \omega\right) \\
v_{s 1, \sigma^{\prime}}(\boldsymbol{r}, \omega)=v_{1, \sigma}(\boldsymbol{r}, \omega)+\sum_{\sigma^{\prime}} \int d^{3} r^{\prime}\left\{\frac{1}{\left\|\boldsymbol{r}-\boldsymbol{r}^{\prime}\right\|}+f_{x c, \sigma \sigma^{\prime}}\left(\boldsymbol{r}, \boldsymbol{r}^{\prime}, \omega\right)\right\} \rho_{1, \sigma^{\prime}}\left(\boldsymbol{r}^{\prime}, \omega\right), \\
\chi_{s, \sigma \sigma^{\prime}}\left(\boldsymbol{r}, \boldsymbol{r}^{\prime}, \omega\right)=\delta_{\sigma \sigma^{\prime}} \sum_{l, k=1}^{\infty}\left(f_{k, \sigma}-f_{l, \sigma}\right) \frac{\psi_{l, \sigma}^{*}(\boldsymbol{r}) \psi_{k, \sigma}(\boldsymbol{r}) \psi_{k, \sigma}^{*}\left(\boldsymbol{r}^{\prime}\right) \psi_{l, \sigma}\left(\boldsymbol{r}^{\prime}\right)}{\omega-\omega_{l k, \sigma}+i \eta}
\end{gathered}
$$

O número de ocupação $f_{i, \sigma}$ diz respeito a ocupação do $i$-ésimo orbital de KS por uma partícula com spin $\sigma$, e as energias de excitação de KS agora carregam o índice de spin,

$$
\omega_{l k, \sigma}=\epsilon_{l, \sigma}-\epsilon_{k, \sigma} .
$$

O kernel de correlação e troca dependente do spin continua sendo dado pela derivada funcional do potencial de correlação com relação a densidade, mas que agora leva em conta as populações de spin, em particular por se tratar

\footnotetext{
${ }^{10}$ As somas são calculadas sobre todos os estados índices $k, l$.
} 
de elétrons,

$$
f_{x c, \sigma \sigma^{\prime}}\left(\boldsymbol{r}, \boldsymbol{r}^{\prime}, t, t^{\prime}\right)=\left.\frac{\delta v_{x c, \sigma}\left[\rho_{\uparrow}, \rho_{\downarrow}\right](\boldsymbol{r}, t)}{\delta \rho_{\sigma^{\prime}}\left(\boldsymbol{r}^{\prime}, t^{\prime}\right)}\right|_{\rho_{0 \uparrow}(\boldsymbol{r}), \rho_{0 \downarrow}(\boldsymbol{r})} .
$$

\subsubsection{As equações de Casida}

Uma vez estabelecido o procedimento de determinação das funções de resposta e portanto dos observáveis (nesse caso a densidade), é de interesse estabelecer uma forma auto-consistente para a obtenção das energias de excitação. Seguindo o procedimento que é detalhado no Apêndice H, chegamos à equação matricial,

$$
\left(\begin{array}{ll}
\mathbb{A} & \mathbb{B} \\
\mathbb{B} & \mathbb{A}
\end{array}\right)\left(\begin{array}{l}
\boldsymbol{X} \\
\boldsymbol{Y}
\end{array}\right)=\Omega\left(\begin{array}{cc}
-\mathbb{1} & 0 \\
0 & \mathbb{1}
\end{array}\right)\left(\begin{array}{l}
\boldsymbol{X} \\
\boldsymbol{Y}
\end{array}\right)
$$

onde os elementos de matriz de $\mathbb{A}$ e $\mathbb{B}$ são dados por,

$$
\begin{gathered}
A_{i a \sigma, i^{\prime} a^{\prime} \sigma^{\prime}}(\Omega)=\delta_{i i^{\prime}} \delta_{a a^{\prime}} \delta_{\sigma \sigma^{\prime}} \omega_{a^{\prime} i^{\prime} \sigma^{\prime}}+K_{i a \sigma, i^{\prime} a^{\prime} \sigma^{\prime}}(\Omega), \\
B_{i a \sigma, i a^{\prime} \sigma^{\prime}}(\Omega)=K_{i a \sigma, i^{\prime} a^{\prime} \sigma^{\prime}}(\Omega) .
\end{gathered}
$$

Os índices $i$ e $i^{\prime}$ dizem respeito a orbitais ocupados e $a$ e $a^{\prime}$ orbitais desocupados, enquanto elementos da matriz de acoplamento $K$ são definidos em termos do kernel de correlação e troca e do termo de Hartree,

$$
K_{i a \sigma, i^{\prime} a^{\prime} \sigma^{\prime}}(\Omega) \equiv \int d^{3} r \int d^{3} r^{\prime} \Phi_{i a, \sigma}(\boldsymbol{r})\left\{\frac{1}{\left\|\boldsymbol{r}-\boldsymbol{r}^{\prime}\right\|}+f_{x c, \sigma \sigma^{\prime}}\left(\boldsymbol{r}, \boldsymbol{r}^{\prime}, \omega\right)\right\} \Phi_{i^{\prime} a^{\prime}, \sigma^{\prime}}^{*}\left(\boldsymbol{r}^{\prime}\right),
$$

onde $\Phi_{i a, \sigma}$ é o produto entre o complexo conjugado de um orbital de KohnSham desocupado e um ocupado,

$$
\Phi_{i a, \sigma}(\boldsymbol{r}) \equiv \psi_{a, \sigma}^{*}(\boldsymbol{r}) \psi_{i, \sigma}(\boldsymbol{r}) .
$$

Os vetores $\boldsymbol{X}$ e $\boldsymbol{Y}$ estão associados a grandezas auxiliares ${ }^{11}$ e são de grande importância para a determinação da correção de primeira ordem na densidade, que é dada por,

$$
\rho_{1, \sigma}(\boldsymbol{r}, \Omega)=\sum_{i, a}\left[\Phi_{i a \sigma}^{*}(\boldsymbol{r}) X_{i a \sigma}(\Omega)+\Phi_{i a \sigma}(\boldsymbol{r}) Y_{i a \sigma}(\Omega)\right]
$$

\footnotetext{
${ }^{11}$ Para mais informações ver Apêndice H.
} 
Do ponto de vista prático, a equação matricial (2.46) é comumente implementada na forma alternativa,

$$
\mathbb{C} \boldsymbol{Z}=\Omega^{2} \boldsymbol{Z}
$$

caracterizando uma equação de autovalores que é satisfeita quando os orbitais de KS são reais e usualmente batizada de equação de Casida. Com um pouco de álgebra é possível extrair que,

$$
\begin{gathered}
\mathbb{C}=(\mathbb{A}-\mathbb{B})^{\frac{1}{2}}(\mathbb{A}+\mathbb{B})(\mathbb{A}-\mathbb{B})^{\frac{1}{2}}, \\
\boldsymbol{Z}=(\mathbb{A}-\mathbb{B})^{\frac{1}{2}}(\boldsymbol{X}-\boldsymbol{Y}) .
\end{gathered}
$$

Utilizando a forma explícita dos elementos de matriz, (2.47) e (2.48), a equação (2.51) assume a forma,

$$
\sum_{i^{\prime} a^{\prime} \sigma^{\prime}}\left[\delta_{i i^{\prime}} \delta_{a a^{\prime}} \delta_{\sigma \sigma^{\prime}} \omega_{a^{\prime} i^{\prime} \sigma}^{2}+2 \sqrt{\omega_{a i \sigma} \omega_{a^{\prime} i^{\prime} \sigma^{\prime}}} K_{i a \sigma, i^{\prime} a^{\prime} \sigma^{\prime}}\right] Z_{i^{\prime} a^{\prime} \sigma^{\prime}}=\Omega^{2} Z_{i a \sigma}
$$

A solução desse conjunto de equações além de exigir a descrição do sistema no estado fundamental, depende do cálculo exato de $f_{x c, \sigma \sigma^{\prime}}\left[\rho_{0}\right]$ e envolve a solução de um problema de autovalores com dimensão infinita. A determinação de $v_{x c}$ e de suas subsequentes ramificações é por construção uma limitação do método, que quando atrelada às restrições computacionais, impossibilita a solução exata das equações acima levando à adotação de diversas aproximações.

Por se tratar de uma metodologia baseada na TD-DFT, a LC-TD-DFTB, também utilizada no presente trabalho, apresenta suas próprias aproximações que são introduzidas na Seção 2.5.

\subsection{DFTB}

Ainda que a DFT seja uma alternativa com baixo custo computacional quando comparado a métodos multideterminantais, o estudo de compostos moleculares extensos e com baixa simetria ainda é dispendioso. Uma das alternativas é o método DFTB, Density Functional based Tight-Binding, que recorre ao formalismo de sistemas fortemente ligados para reduzir, tipicamente, 2 a 3 ordens de grandeza do tempo de cálculo. Considerando 
um sistema de $N$ elétrons, o custo computacional escala com $N^{2}$ para cálculos usando DFTB, em oposição a $N^{3}$ do DFT e $N^{5}$ de métodos pósHF como MP2 [70, 71].

\section{As hipóteses do modelo}

Partindo das ideias introduzidas por Thomas Frauenheim et al. em 1995 [72], o método utiliza do formalismo de Kohn-Sham e escreve a energia como funcional da densidade eletrônica de acordo com a equação (2.22). Sendo $n_{i}$ o número de ocupação, ao escrever $\epsilon_{i}$ como elemento de matriz, a energia pode ser expressa como,

$$
\begin{aligned}
E[\rho]= & \sum_{i} n_{i}\left\langle\psi_{i}\left|\left(-\frac{\nabla^{2}}{2}+v(\boldsymbol{r})+\int d \boldsymbol{r}^{\prime} \frac{\rho\left(\boldsymbol{r}^{\prime}\right)}{\left\|\boldsymbol{r}-\boldsymbol{r}^{\prime}\right\|}+v_{x c}(\boldsymbol{r})\right)\right| \psi_{i}\right\rangle \\
& -\frac{1}{2} \iint d \boldsymbol{r} d \boldsymbol{r}^{\prime} \frac{\rho(\boldsymbol{r}) \rho\left(\boldsymbol{r}^{\prime}\right)}{\left\|\boldsymbol{r}-\boldsymbol{r}^{\prime}\right\|}+E_{x c}[\rho(\boldsymbol{r})]-\int d \boldsymbol{r} v_{x c}(\boldsymbol{r}) \rho(\boldsymbol{r}) .
\end{aligned}
$$

É nesse momento que nos despedimos definitivamente da descrição $a b$ initio. Inspirado nos orbitais localizados do modelo de elétrons fortemente ligados (Tight-binding), considere um sistema com densidade $\rho_{0}(\boldsymbol{r})$ composta por densidades atômicas, como se os átomos fossem neutros e livres. Suponha que a densidade que minimiza o funcional de energia é suficientemente próxima da dos átomos livres, tal que,

$$
\rho_{\min }(\boldsymbol{r})=\rho_{0}(\boldsymbol{r})+\Delta \rho(\boldsymbol{r}),
$$

onde $\Delta \rho_{0}(\boldsymbol{r})$ é uma contribuição pequena. Substituindo (2.53) na expressão do funcional de energia (2.52) e expandindo o funcional de correlação e troca $E_{x c}\left[\rho_{0}(\boldsymbol{r})+\Delta \rho(\boldsymbol{r})\right]$ em uma série de Taylor até a terceira ordem (ver Apêndice I), obtemos que, 


$$
\begin{aligned}
E\left[\rho_{0}(\boldsymbol{r})+\Delta \rho(\boldsymbol{r})\right]= & \sum_{i} n_{i}\left\langle\psi_{i}\left|\hat{H}_{0}\right| \psi_{i}\right\rangle-\frac{1}{2} \iint d \boldsymbol{r} d \boldsymbol{r}^{\prime} \frac{\rho_{0}(\boldsymbol{r}) \rho_{0}\left(\boldsymbol{r}^{\prime}\right)}{\left\|\boldsymbol{r}-\boldsymbol{r}^{\prime}\right\|}+E_{x c}\left[\rho_{0}(\boldsymbol{r})\right] \\
& -\int d \boldsymbol{r} v_{x c}(\boldsymbol{r}) \rho_{0}(\boldsymbol{r}) \\
& +\frac{1}{2} \iint d \boldsymbol{r} d \boldsymbol{r}^{\prime}\left(\frac{1}{\left\|\boldsymbol{r}-\boldsymbol{r}^{\prime}\right\|}+\left[\frac{\delta^{2} E_{x c}[\rho(\boldsymbol{r})]}{\delta \rho(\boldsymbol{r}) \delta \rho\left(\boldsymbol{r}^{\prime}\right)}\right]_{\rho=\rho_{0}}\right) \Delta \rho(\boldsymbol{r}) \Delta \rho\left(\boldsymbol{r}^{\prime}\right) \\
& +\frac{1}{6} \iiint d \boldsymbol{r} d \boldsymbol{r}^{\prime} d \boldsymbol{r}^{\prime \prime}\left[\frac{\delta^{3} E_{x c}[\rho(\boldsymbol{r})]}{\delta \rho(\boldsymbol{r}) \delta \rho\left(\boldsymbol{r}^{\prime}\right) \delta \rho\left(\boldsymbol{r}^{\prime \prime}\right)}\right]_{\rho=\rho_{0}} \Delta \rho(\boldsymbol{r}) \Delta \rho\left(\boldsymbol{r}^{\prime}\right) \Delta \rho\left(\boldsymbol{r}^{\prime \prime}\right)
\end{aligned}
$$

onde os termos da primeira somatória correspondem aos elementos de matriz da hamiltoniana de Kohn-Sham sujeita a densidade atômica $\rho_{0}(\boldsymbol{r})$,

$$
\hat{H}_{0} \equiv \hat{h}_{\rho_{0}}^{K S}=-\frac{\nabla^{2}}{2}+v(\boldsymbol{r})+\int d \boldsymbol{r}^{\prime} \frac{\rho_{0}\left(\boldsymbol{r}^{\prime}\right)}{\left\|\boldsymbol{r}-\boldsymbol{r}^{\prime}\right\|}+v_{x c}(\boldsymbol{r})
$$

\subsubsection{A variante SCC-DFTB}

Na literatura, a ordem de expansão da energia de correlação e troca define a nomenclatura do método, DFTB1 para primeira ordem, DFTB3 para a terceira e assim sucessivamente. Em particular, ao truncar a série nos termos quadráticos o método é popularmente chamado de SCC-DFTB, Selfconsistent Charge Corrected Density Functional Tight-Binding pois, entre outras coisas, os termos de segunda ordem trazem as primeiras correções na distribuição de carga ao longo do sistema, quebrando a neutralidade dos átomos. Dentro desse limite, as componentes do funcional (2.54) são separadas em três partes usualmente referidas como, a energia da estrutura de banda,

$$
E_{B S}[\rho(\boldsymbol{r})]=\sum_{i} n_{i}\left\langle\psi_{i}\left|\hat{H}_{0}\right| \psi_{i}\right\rangle
$$

dada pela soma dos autovalores dos orbitais ocupados, a energia de repulsão,

$$
E_{r e p}[\rho(\boldsymbol{r})]=-\frac{1}{2} \iint d \boldsymbol{r} d \boldsymbol{r}^{\prime} \frac{\rho_{0}(\boldsymbol{r}) \rho_{0}\left(\boldsymbol{r}^{\prime}\right)}{\left\|\boldsymbol{r}-\boldsymbol{r}^{\prime}\right\|}+E_{x c}\left[\rho_{0}(\boldsymbol{r})\right]-\int d \boldsymbol{r} v_{x c}(\boldsymbol{r}) \rho_{0}(\boldsymbol{r})+E_{n n},
$$

e a correção de segunda ordem, 


$$
E_{2 n d}[\rho(\boldsymbol{r})]=\frac{1}{2} \iint d \boldsymbol{r} d \boldsymbol{r}^{\prime}\left(\frac{1}{\left\|\boldsymbol{r}-\boldsymbol{r}^{\prime}\right\|}+\left[\frac{\delta^{2} E_{x c}[\rho(\boldsymbol{r})]}{\delta \rho(\boldsymbol{r}) \delta \rho\left(\boldsymbol{r}^{\prime}\right)}\right]_{\rho=\rho_{0}}\right) \Delta \rho(\boldsymbol{r}) \Delta \rho\left(\boldsymbol{r}^{\prime}\right),
$$

também chamada de energia de Coulomb, por ter sua principal contribuição decorrente do termo $\left\|\boldsymbol{r}-\boldsymbol{r}^{\prime}\right\|^{-1}$.

\subsubsection{Energia da estrutura de banda e o pseudo-átomo}

A primeira contribuição de ordem zero (2.56) é familiar da DFT, sendo determinada pela soma de elementos de matriz do operador (2.55). O detalhe diz respeito à determinação dos orbitais. É adotado o procedimento usual de expandir os orbitais $\psi_{i}$ como combinação linear de funções convenientes. Ainda que não utilizemos funções gaussianas, assim como na expansão discutida na Seção 2.2.4, os orbitais são descritos a partir da base mínima de funções de Slater,

$$
\psi_{i}(\boldsymbol{r})=\sum_{\mu} c_{\mu}^{i} \varphi_{\mu}(\boldsymbol{r})=\sum_{\mu} c_{\mu}^{i} R_{\mu}(r) Y_{\mu}(\theta, \phi)
$$

onde $Y_{\mu}$ são os harmônicos esféricos ${ }^{12}$ e $R_{\mu}$ as funções radiais. As soluções da equação de Kohn-Sham sujeita a densidade $\rho_{0}(\boldsymbol{r})$ resultam em orbitais difusos, e devido a hipótese de elétrons fortemente ligados, é adicionado um potencial de confinamento, da forma ${ }^{13}$,

$$
V_{\text {conf }}(r)=\sum_{i=1}^{\infty} v_{2 i} r^{2 i}
$$

responsável por encurtar a cauda difusa, formando uma base compacta de orbitais pseudo-atômicos, tipicamente mais apropriada para a expansão da função de onda. As constantes $v_{2 i}$ são tratadas como parâmetros e ajustadas. Como exemplo, considerando apenas o primeiro termo da expansão,

$$
V_{\text {conf }}(r)=v_{2} r^{2} \equiv\left(\frac{r}{2 r_{\text {cov }}}\right)^{2},
$$

onde $r_{c o v}$ é o raio covalente do átomo no qual o potencial está centrado e

\footnotetext{
${ }^{12} \mathrm{E}$ usual a escolha de harmônicos esféricos reais, pois promove simplificações como a simetrização da matriz de overlap.

${ }^{13}$ As potências ímpares não são consideradas pois impomos que o potencial seja bem comportado na origem.
} 
2 é um fator empírico. Essa escolha apresenta bons resultados e serve de ponto de partida para a parametrização de $v_{2}$ e subsequente determinação de $E_{B S}$.

\subsubsection{Correção de segunda ordem}

Considere o espaço $\mathbb{V}$ subdividido em volumes em torno de cada átomo, de tal forma que,

$$
\sum_{I} \mathbb{V}_{I}=\mathbb{V} \quad \text { e } \int_{\mathbb{V}}=\sum_{I} \int_{\mathbb{V}_{I}},
$$

onde $\mathbb{V}_{I}$ é o volume em torno do $I$-ésimo átomo. No contexto de elétrons localizados, a variação de carga elétrica em torno do I-ésimo átomo pode ser aproximada por,

$$
\Delta q_{I} \approx \int_{\mathbb{V}_{I}} \Delta \rho(\boldsymbol{r}) d \boldsymbol{r}
$$

e a variação na densidade pode ser expressa a partir das contribuições atômicas,

$$
\Delta \rho(\boldsymbol{r})=\sum_{I} \Delta q_{I} \Delta \rho_{I}(\boldsymbol{r}),
$$

sendo $\Delta \rho_{I}(\boldsymbol{r})$ a variação da densidade no volume $\mathbb{V}_{I}$. Utilizando essa decomposição, a correção de segunda ordem (2.58) é reduzida à soma de integrais sobre pares atômicos,

$$
E_{2 n d}[\rho(\boldsymbol{r})]=\frac{1}{2} \sum_{I J} \Delta q_{I} \Delta q_{J} \int_{\mathbb{V}_{I}} \int_{\mathbb{V}_{J}} d \boldsymbol{r} d \boldsymbol{r}^{\prime}\left(\frac{1}{\left\|\boldsymbol{r}-\boldsymbol{r}^{\prime}\right\|}+\left[\frac{\delta^{2} E_{x c}[\rho(\boldsymbol{r})]}{\delta \rho(\boldsymbol{r}) \delta \rho\left(\boldsymbol{r}^{\prime}\right)}\right]_{\rho=\rho_{0}}\right) \Delta \rho_{I}(\boldsymbol{r}) \Delta \rho_{J}\left(\boldsymbol{r}^{\prime}\right) .
$$

Quando $I=J$, seguindo a expansão da energia atômica em potências de $\Delta q_{I}$, a integral em (2.60) é aproximada pelo parâmetro Hubbard $U$,

$$
U=2 \eta=\frac{\partial^{2} E}{\partial n^{2}} \approx I P-E A
$$

onde $\eta$ é a dureza absoluta do átomo, $n$ o número de ocupação, IE a energia de ionização e $E A$ a afinidade eletrônica. Para $I \neq J$ e no caso de um potencial local a derivada de segunda ordem é proporcional a delta de Dirac, 


$$
\frac{\delta^{2} E_{x c}[\rho(\boldsymbol{r})]}{\delta \rho(\boldsymbol{r}) \delta \rho\left(\boldsymbol{r}^{\prime}\right)} \propto \delta\left(\boldsymbol{r}-\boldsymbol{r}^{\prime}\right),
$$

restando apenas a contribuição coulombiana no termo de segunda ordem,

$$
E_{2 n d}[\rho(\boldsymbol{r})]=\frac{1}{2} \sum_{I J} \Delta q_{I} \Delta q_{J} \int_{\mathbb{V}_{I}} \int_{\mathbb{V}_{J}} d \boldsymbol{r} d \boldsymbol{r}^{\prime} \frac{\Delta \rho_{I}(\boldsymbol{r}) \Delta \rho_{J}\left(\boldsymbol{r}^{\prime}\right)}{\left\|\boldsymbol{r}-\boldsymbol{r}^{\prime}\right\|}
$$

Entre as possibilidades de modelagem das variações nas distribuições de carga atômicas, as duas mais usuais envolvem gaussianas centradas nos átomos ou expansões em termos de funções radiais e harmônicos esféricos. No primeiro caso,

$$
\Delta \rho_{I}(\boldsymbol{r})=\frac{e^{\frac{-\left(\boldsymbol{r}-\boldsymbol{R}_{I}\right)^{2}}{2 \sigma_{I}^{2}}}}{\sqrt{\left(2 \pi \sigma_{I}\right)^{3}}}
$$

onde a largura $\sigma_{I}$ é ajustada em termos de $U$, de tal forma a reproduzir a calda coulombiana para $R \gg 0$ e tender a $U$ quando $R \rightarrow 0$. Como o produto de duas gaussianas também é uma gaussiana, a integral apresentada na equação (2.62), batizada de $\gamma_{I J}$, assume a forma,

$$
\gamma_{I J}=\left\{\begin{array}{cc}
U_{I}, & I=J \\
\frac{\operatorname{erf}\left(C_{I} R_{I J}\right)}{R_{I J}}, & I \neq J
\end{array},\right.
$$

sendo erf( $x)$ a função erro e $R_{I J} \equiv\left\|\boldsymbol{R}_{I}-\boldsymbol{R}_{J}\right\|$. O outro modelo, implementado no código DFTB + , expande $\Delta \rho_{I}(\boldsymbol{r})$ em produtos de funções radiais e harmônicos esféricos e trunca a expansão no primeiro termo. A função radial consiste na função de Slater que caracteriza a solução esfericamente simétrica do átomo de hidrogênio, de tal forma que,

$$
\begin{aligned}
\Delta \rho_{I}(\boldsymbol{r}) & =\sum_{l, m} K_{l m} R_{n, l}\left(\left\|\boldsymbol{r}-\boldsymbol{R}_{I}\right\|\right) Y_{l m}\left(\frac{\boldsymbol{r}-\boldsymbol{R}_{I}}{\left\|\boldsymbol{r}-\boldsymbol{R}_{I}\right\|}\right) \\
& \left.\simeq K_{00} R_{10}\left(\left\|\boldsymbol{r}-\boldsymbol{R}_{I}\right\|\right) Y_{00}\left(\frac{\boldsymbol{r}-\boldsymbol{R}_{I}}{\left\|\boldsymbol{r}-\boldsymbol{R}_{I}\right\|}\right)\right) \\
& \propto e^{-\alpha_{I}\left\|\boldsymbol{r}-\boldsymbol{R}_{I}\right\|} .
\end{aligned}
$$




\subsubsection{Energia de repulsão}

O termo de repulsão, formalmente introduzido como (2.57), exerce papel equivalente ao do potencial de correlação e troca na DFT, sendo responsável pelas contribuições desconhecidas do sistema e portanto estabelecendo as limitações do modelo. Uma vez que cada uma das contribuições é dada em termos da densidade esfericamente simétrica dos átomos livres, é razoável admitir que interações entre 3 ou mais corpos possuem contribuições desprezíveis frente as contribuições de 2 corpos, e portanto o potencial é aproximado pela soma de elementos entre pares atômicos,

$$
E_{\text {rep }}=\sum_{I<J} V_{\text {rep }}^{I J}\left(\left\|\boldsymbol{R}_{I}-\boldsymbol{R}_{J}\right\|\right) .
$$

As parametrizações variam de acordo com o grupo responsável pelo desenvolvimento mas têm como origem a minimização, introduzida em 1995 [72], da diferença entre as energias do estado fundamental obtidas via DFTB e DFT. Devido a aproximação de interações de pares, o potencial é ajustado por meio de cálculos envolvendo dímeros que varrem um intervalo de distâncias interatômicas. O conjunto OB2 [70], utilizado no presente trabalho, tem o potencial de repulsão expresso por,

$$
V_{r e p}^{I J}\left(R_{I J}\right)=\left\{\begin{array}{cc}
e^{-a_{1} R_{I J}+a_{2}}+a_{3}, & R_{I J}<R_{I J, 0} \\
\sum_{i=0}^{k} a_{I J, n, i}\left(R_{I J}-R_{I J, n}\right)^{i}, & R_{I J, n} \leq R_{I J}<R_{I J, n+1} \\
0, & R_{I J} \geq R_{I J, \text { corte }} .
\end{array}\right.
$$

Para distâncias menores que o raio de corte $R_{I J, c o r t e}$, o intervalo é dividido em $n$ subintervalos nos quais o potencial é descrito por polinômios de ordem $k$, e um subintervalo $\left(R_{I J}<R_{I J, 0}\right)$ que mimetiza o comportamento da interação nas proximidades da origem por meio de uma exponencial decrescente. Na equação $(2.64), a_{m}$ são constantes e $R_{I J, 0}$ diz respeito ao limite a direita do primeiro intervalo, $R_{I J, 1}$ do segundo intervalo e assim sucessivamente. Uma vez estabelecido o potencial, $a_{m}, n, R_{I J, n}$ e $k$ são ajustados de tal forma a minimizar a função, 


$$
\begin{aligned}
f^{s c o r e}= & \sum_{i} W_{a t, i}\left|E_{a t, i}^{r e f}-E_{a t, i}^{D F T B}\right|+\sum_{i} W_{b a r, i}\left|E_{b a r, i}^{r e f}-E_{b a r, i}^{D F B}\right|+ \\
& +\sum_{i} W_{F, i} \sum_{j}\left|F_{i, j}^{D F T B}\right|+\sum_{i} W_{F, i} \sum_{j}\left|F_{i, j}^{r e f}-F_{i, j}^{D F T B}\right|,
\end{aligned}
$$

que leva em consideração a diferença das energias de atomização $\left(E_{a t, i}\right)$ de referência e as obtidas via DFTB, assim como as diferenças entre as barreiras de transferência de prótons $\left(E_{b a r, i}\right)$, módulo das forças na geometria do estado fundamental $\left(F_{i, j}\right)$ e o valor absoluto das forças obtidas via DFTB. Cada uma delas ponderada pelo respectivo peso $W_{k, i}$ que a priori é fixado como 1, atribuindo a mesma relevância para cada grandeza.

\subsubsection{Princípio variacional}

Contabilizando os modelos introduzidos para cada uma das contribuições e tendo em vista a expansão em orbitais atômicos (2.59), a energia total pode ser expressa por,

$$
\begin{aligned}
E[\rho(\boldsymbol{r})]= & \sum_{i} n_{i} \sum_{\mu, \nu} c_{\mu}^{i *} c_{\nu}^{i}\left\langle\varphi_{\mu}\left|\hat{H}_{0}\right| \varphi_{\nu}\right\rangle \\
& +\frac{1}{2} \sum_{I, J} \gamma_{I J}\left(\left\|\boldsymbol{R}_{I}-\boldsymbol{R}_{J}\right\|\right) \Delta q_{I} \Delta q_{J}+\sum_{I<J} V_{r e p}^{I J}\left(\left\|\boldsymbol{R}_{I}-\boldsymbol{R}_{J}\right\|\right) .
\end{aligned}
$$

Seguindo a mesma abordagem que leva às equações de Kohn-Sham, ao aplicar o método dos multiplicadores de Lagrange atrelado a condição de ortogonalidade dos spin-orbitais atômicos, com o intuito de minimizar o funcional de energia, ou seja, partindo de (2.19) mas agora com o funcional de energia (2.66) e os spin-orbitais (2.59) do pseudo-átomo, obtemos um conjunto de equações de autoestados e autovalores,

$$
\sum_{\nu} c_{\nu}^{i}\left(H_{\mu \nu}-\varepsilon_{i} S_{\mu \nu}\right)=0
$$

onde $S$ é a matriz de overlap, tal que, 


$$
S_{\mu \nu}=\int d \boldsymbol{r} \varphi_{\mu}^{*}(\boldsymbol{r}) \varphi_{\nu}(\boldsymbol{r})
$$

e $H$ a hamiltoniana de KS do átomo livre (2.55), sujeita a correções de caráter eletrostático,

$$
\begin{aligned}
H_{\mu \nu} & =\left(\hat{H}_{0}\right)_{\mu \nu}+\frac{1}{2} S_{\mu \nu} \sum_{K}\left(\gamma_{I K}+\gamma_{J K}\right) \Delta q_{K} \\
& =\left(\hat{H}_{0}\right)_{\mu \nu}+\frac{1}{2} S_{\mu \nu}\left(\epsilon_{I}+\epsilon_{J}\right)
\end{aligned}
$$

sendo $\epsilon_{I}$ o potencial eletrostático sentido pelo $I$-ésimo átomo devido a flutuação das cargas atômicas nos demais átomos. A correção é interpretada como um deslocamento na hamiltoniana em decorrência do potencial eletrostático médio ao redor dos orbitais vizinhos $\mu$ e $\nu$, levando a nomenclatura Self-consistent Charge Corrected para a expansão DFTB2.

É importante destacar que o operador $\hat{H}_{0}$ é calculado a partir de $(2.55)$ e portanto utiliza de um funcional de correlação e troca. No código DFTB+ $[35,36]$ está implementado o funcional LC-BNL [73, 74], de tal forma que os elementos de matriz $\left(\hat{H}_{0}\right)_{\mu \nu}$, assim como $S_{\mu \nu}$ são previamente calculados para um conjunto de distâncias interatômicas [70]. Esses valores são reunidos com as integrais $\gamma_{I J}$ e o resultado da parametrização do potencial de repulsão para compor os parâmetros de Slater-Koster. A perda da flexibilidade frente a escolha de $E_{x c}$ e da base atômica resulta no ganho computacional, uma vez que boa parte das integrais não precisam ser calculadas.

\subsubsection{Correção de longo alcance}

Com a mesma proposta da DFT, as correções de longo alcance buscam minimizar erros de autointeração e de delocalização da densidade eletrônica, incorporando flexibilidade no potencial coulombiano entre os elétrons. No software DFTB+ a correção é baseada na decomposição de Yukawa, distinta da (2.23) utilizada no funcional $\omega$ B97X-D,

$$
\frac{1}{\left\|\boldsymbol{r}-\boldsymbol{r}^{\prime}\right\|}=\frac{e^{-\omega\left\|\boldsymbol{r}-\boldsymbol{r}^{\prime}\right\|}}{\left\|\boldsymbol{r}-\boldsymbol{r}^{\prime}\right\|}+\frac{1-e^{-\omega\left\|\boldsymbol{r}-\boldsymbol{r}^{\prime}\right\|}}{\left\|\boldsymbol{r}-\boldsymbol{r}^{\prime}\right\|}
$$

onde $\omega$ é o parâmetro de longo alcance e $\left\|\boldsymbol{r}-\boldsymbol{r}^{\prime}\right\|$ é a distância entre os elétrons 1 e 2. Seguindo a correção proposta por Baer e Neuhauser [73], o 
termo de correlação e troca do funcional BNL é dado por,

$$
E_{x c}=\underbrace{E_{c}^{P B E}+\alpha E_{x}^{\omega, L D A}}_{E_{x c}^{\omega, D F T}}+E_{x}^{\omega, H F} .
$$

O parâmetro empírico $\alpha$ varia de 0 até 1 e é calibrado a partir de dados experimentais, de acordo com a propriedade de interesse. O funcional de correlação adotado é o do PBE [75] e o parâmetro foi fixado como $\alpha=1$ [35]. A energia de troca do gás elétrons homogêneo sujeito a interação de Yukawa assume a forma [76],

$$
E_{x}^{\omega, L D A}=-\frac{3}{2}\left(\frac{3}{4 \pi}\right)^{\frac{1}{3}} \sum_{\sigma} \int d \boldsymbol{r} \rho_{\sigma}^{4 / 3}(\boldsymbol{r}) F\left(a_{\sigma, \omega}\right),
$$

onde $\sigma$ soma sobre as projeções de spin $\alpha$ e $\beta$, com,

$$
F\left(a_{\sigma, \omega}\right)=1-\frac{8 a_{\sigma, \omega}}{3}\left\{\arctan \left(a_{\sigma, \omega}^{-1}\right)+\frac{a_{\sigma, \omega}}{4}\left[1-\left(a_{\sigma, \omega}^{2}+3\right) \ln \left(1+\frac{1}{a_{\sigma, \omega}^{2}}\right)\right]\right\}
$$

em função do parâmetro,

$$
a_{\sigma, \omega}=\frac{\omega}{2\left[3 \pi^{2} \rho_{\sigma}(\boldsymbol{r})\right]^{\frac{1}{3}}} .
$$

O termo $E_{x}^{\omega, H F}$ se aproxima da energia exata de Hartree-Fock no limite em que as distâncias interatômicas são grandes, sendo dado por,

$$
E_{x}^{\omega, H F}=-\frac{1}{2} \sum_{\sigma} \sum_{i, j} \iint d \boldsymbol{r} d \boldsymbol{r}^{\prime} \psi_{i \sigma}^{*}(\boldsymbol{r}) \psi_{j \sigma}^{*}(\boldsymbol{r})\left(\frac{1-e^{-\omega\left\|\boldsymbol{r}-\boldsymbol{r}^{\prime}\right\|}}{\left\|\boldsymbol{r}-\boldsymbol{r}^{\prime}\right\|}\right) \psi_{i \sigma}\left(\boldsymbol{r}^{\prime}\right) \psi_{j \sigma}\left(\boldsymbol{r}^{\prime}\right) .
$$

Note que essa correção incorpora apenas o termo de longo alcance $\left(E_{x}^{\omega, H F} \equiv\right.$ $\left.E_{x}^{\mathrm{LR}-\mathrm{HF}}\right)$, de tal forma que o termo de curto alcance é puramente local $\left(E_{x}^{\omega, L D A}\right)$. De posse do funcional adaptado, é realizado o mesmo procedimento discutido anteriormente, com o detalhe de que a expansão em polinômios de Taylor é realizada apenas para o termo $E_{x c}^{\omega, D F T}$. Truncando a somatória em segunda ordem, no espírito do formalismo SCC, e simplificando os resultados por meio da matriz de densidade na representação dos 
orbitais atômicos, ${ }^{14}$

$$
(\boldsymbol{P})_{\mu, \nu} \equiv P_{\mu \nu}=2 \sum_{i=1}^{N / 2} n_{i} c_{\nu, i} c_{\mu, i}
$$

onde $c_{\kappa, i}$ diz respeito ao coeficiente da $\kappa$-ésima função de base do $i$-ésimo orbital e $n_{i}$ corresponde ao número de ocupação desse orbital. Ao reorganizar os elementos [36], o funcional de energia passa a ser,

$$
\begin{aligned}
E= & \sum_{\mu, \nu} P_{\mu \nu} H_{\mu \nu}^{0}+\frac{1}{2} \sum_{\mu \nu \alpha \beta} \Delta P_{\mu \nu} \Delta P_{\alpha \beta}\left[(\mu \nu \mid \alpha \beta)+f_{\mu \nu \alpha \beta}^{\omega, x c}\left[\rho_{0}\right]\right] \\
& -\frac{1}{4} \sum_{\mu \nu \alpha \beta} \Delta P_{\mu \nu} \Delta P_{\alpha \beta}(\mu \alpha \mid \beta \nu)^{l r}+E_{r e p} .
\end{aligned}
$$

A hamiltoniana $H_{\mu \nu}^{0}$ é igual a (2.55) com a adição do termo de longo alcance e a mudança de $v_{x c}$ para a derivada funcional do potencial $E_{x c}^{\omega, D F T}$,

$$
H_{\mu \nu}^{0}=h_{\mu \nu}+\sum_{\alpha \beta} P_{\alpha \beta}^{0}(\mu \nu \mid \alpha \beta)-\frac{1}{2} \sum_{\alpha \beta} P_{\alpha \beta}^{0}(\mu \alpha \mid \beta \nu)^{l r}+v_{\mu \nu}^{\omega, x c}\left[\rho_{0}\right] .
$$

O termo $P_{\alpha \beta}^{0}$ faz referência à matriz de densidade dos átomos livres, enquanto o elemento de matriz $h_{\mu \nu}$ e as integrais de dois elétrons são dados por,

$$
\begin{aligned}
h_{\mu \nu} & =\int d \boldsymbol{r} \varphi_{\mu}^{*}(\boldsymbol{r})\left(-\frac{\nabla^{2}}{2}+v(\boldsymbol{r})\right) \varphi_{\nu}(\boldsymbol{r}) \\
(\mu \nu \mid \alpha \beta) & =\iint d \boldsymbol{r} d \boldsymbol{r}^{\prime} \varphi_{\mu}^{*}(\boldsymbol{r}) \varphi_{\nu}^{*}(\boldsymbol{r})\left(\frac{1}{\left\|\boldsymbol{r}-\boldsymbol{r}^{\prime}\right\|}\right) \varphi_{\mu}\left(\boldsymbol{r}^{\prime}\right) \varphi_{\nu}\left(\boldsymbol{r}^{\prime}\right) \\
(\mu \nu \mid \alpha \beta)^{l r} & =\iint d \boldsymbol{r} d \boldsymbol{r}^{\prime} \varphi_{\mu}^{*}(\boldsymbol{r}) \varphi_{\nu}^{*}(\boldsymbol{r})\left(\frac{1-e^{-\omega\left\|\boldsymbol{r}-\boldsymbol{r}^{\prime}\right\|}}{\left\|\boldsymbol{r}-\boldsymbol{r}^{\prime}\right\|}\right) \varphi_{\mu}\left(\boldsymbol{r}^{\prime}\right) \varphi_{\nu}\left(\boldsymbol{r}^{\prime}\right) .
\end{aligned}
$$

A energia $E_{\text {rep }}$ continua sendo ajustada seguindo o procedimento elucidado na Subseção 2.4 .4 e $f_{\mu \nu \alpha \beta}^{\omega, x c}\left[\rho_{0}\right]$ diz respeito à segunda derivada do potencial $E_{x c}^{\omega, D F T}$ calculada em $\rho=\rho_{0}$ na base dos orbitais atômicos.

Uma vez estabelecido o funcional com correção de longo alcance, via (2.72),

\footnotetext{
${ }^{14}$ No caso de sistemas abertos a generalização é imediata por meio da somatória de $i$ até $N$ e sobre as projeções de spin.
} 
as mesmas hipóteses de primeiros vizinhos são realizadas, com destaque para as aproximações de 2 e 4 centros para as integrais (2.67) e (2.73), possibilitando a utilização das regras de Slater-Koster e subsequentes parametrizações. O princípio variacional é empregado, resultando no procedimento auto-consistente que dispõe dos elementos de matriz de $H^{0}$ e $S$ calculados previamente, reduzindo o custo computacional e facilitando a convergência dos resultados.

\subsection{LC-TD-DFTB}

A variante dependente do tempo utiliza do formalismo de resposta linear assim como na DFT. Seguindo a mesma construção discutida na Seção 2.3, por meio da resposta linear da densidade eletrônica frente a perturbação que acopla com o operador de densidade, chegamos na equação matricial de Casida, cujos autoestados determinam as densidades de transição e respectivas forças de oscilador, enquanto os autovalores correspondem as energias de transição associadas.

Nessa seção serão incorporadas as aproximações da metodologia LC-DFTB nas equações de Casida, em particular, a construção da matriz de acoplamento $K(\Omega)$ que carrega o kernel $f_{x c}$, expresso em termos da derivada de segunda ordem do funcional de correlação e troca.

\subsubsection{Generalização para sistemas de camada aberta}

Por meio da adição de spin é possível generalizar os resultados apresentados para sistemas de camada aberta, além de facilitar a conexão com a TD-DFT. O formalismo implementado no código DFTB+ é apresentado no artigo de 2017 de Kranz et al. [36] e parte da equação matricial (2.46) sujeita a uma alteração de sinal nas definições de $\boldsymbol{X}$ e $\boldsymbol{Y}$ que resulta em,

$$
\left(\begin{array}{ll}
\mathbb{A} & \mathbb{B} \\
\mathbb{B} & \mathbb{A}
\end{array}\right)\left(\begin{array}{l}
\boldsymbol{X} \\
\boldsymbol{Y}
\end{array}\right)=\Omega\left(\begin{array}{cc}
\mathbb{1} & 0 \\
0 & -\mathbb{1}
\end{array}\right)\left(\begin{array}{l}
\boldsymbol{X} \\
\boldsymbol{Y}
\end{array}\right) .
$$

Os elementos de matriz de $\mathbb{A}$ e $\mathbb{B}$ continuam sendo dados respectivamente pelas equações (2.47) e (2.48), mas agora a matriz de acoplamento $K(\Omega)$ deriva a hamiltoniana LC-DFTB, de tal forma que,

$$
K_{i a \sigma, i^{\prime} a^{\prime} \sigma^{\prime}}(\Omega)=\frac{\partial H_{i a \sigma}}{\partial P_{i^{\prime} a^{\prime} \sigma^{\prime}}}
$$


onde, ${ }^{15}$

$$
\begin{aligned}
H_{i a \sigma}= & H_{\mu \nu}^{0}+\sum_{\tau} \sum_{\alpha \beta} \Delta P_{\alpha \beta \tau}\left[(\mu \nu \mid \alpha \beta)+f_{\mu \nu \sigma, \alpha \beta \tau}^{\omega, x c}\left[\rho_{0}\right]\right] \\
& -\frac{1}{2} \sum_{\alpha \beta} \Delta P_{\alpha \beta \sigma}(\mu \alpha \mid \beta \nu)^{l r} .
\end{aligned}
$$

A notação por mais que seja carregada é mantida dessa forma pois facilita a comparação. O termo de acoplamento (2.75) é equivalente a (2.49) com a adição da hamiltoniana livre $H_{\mu \nu \sigma}^{0}$ e a correção de longo alcance, dada pela somatória das integrais $(\mu \alpha \mid \beta \nu)^{l r}$.

\subsubsection{Adaptação das equações de Casida}

As integrais de 4 centros que compõem a hamiltoniana (2.76) são tratadas na aproximação de Mulliken [35],

$$
\varphi_{\mu}(\boldsymbol{r}) \varphi_{\nu}(\boldsymbol{r}) \approx \frac{1}{2} S_{\mu \nu}\left[\left|\varphi_{\mu}(\boldsymbol{r})\right|^{2}+\left|\varphi_{\nu}(\boldsymbol{r})\right|^{2}\right]
$$

e reduzidas a soma de integrais de 2 elétrons,

$$
\begin{aligned}
(\mu \nu \mid \alpha \beta) & \approx \frac{1}{4} S_{\mu \nu} S_{\alpha \beta}[(\mu \mu \mid \alpha \alpha)+(\mu \mu \mid \beta \beta)+(\nu \nu \mid \alpha \alpha)+(\nu \nu \mid \beta \beta)] \\
& \equiv \frac{1}{4} S_{\mu \nu} S_{\alpha \beta}\left[\gamma_{\mu \alpha}+\gamma_{\mu \beta}+\gamma_{\nu \alpha}+\gamma_{\nu \beta}\right], \\
(\mu \alpha \mid \beta \nu)^{l r} & \approx \frac{1}{4} S_{\mu \alpha} S_{\beta \nu}\left[(\mu \mu \mid \beta \beta)^{l r}+(\mu \mu \mid \nu \nu)^{l r}+(\alpha \alpha \mid \beta \beta)^{l r}+(\alpha \alpha \mid \nu \nu)^{l r}\right] \\
& \equiv \frac{1}{4} S_{\mu \alpha} S_{\beta \nu}\left[\gamma_{\mu \beta}^{l r}+\gamma_{\mu \nu}^{l r}+\gamma_{\alpha \beta}^{l r}+\gamma_{\alpha \nu}^{l r}\right],
\end{aligned}
$$

\footnotetext{
${ }^{15}$ A hamiltonina de referência $H_{\mu \nu}^{0}$ é escolhida como sendo de camada fechada $\left(\boldsymbol{P}_{\sigma=\uparrow}^{\mathbf{0}}=\boldsymbol{P}_{\sigma=\downarrow}^{\mathbf{0}}\right)$.
} 


$$
\begin{aligned}
f_{\mu \nu \sigma, \alpha \beta \tau}^{\omega, x c}= & \left(\mu \nu\left|f_{\sigma, \tau}^{\omega, x c}\left[\rho_{0}\right]\right| \alpha \beta\right) \\
\approx & \frac{1}{4} S_{\mu \nu} S_{\alpha \beta}\left[\left(\mu \mu\left|f_{\sigma, \tau}^{\omega, x c}\left[\rho_{0}\right]\right| \alpha \alpha\right)+\left(\mu \mu\left|f_{\sigma, \tau}^{\omega, x c}\left[\rho_{0}\right]\right| \beta \beta\right)+\right. \\
& \left.+\left(\nu \nu\left|f_{\sigma, \tau}^{\omega, x c}\left[\rho_{0}\right]\right| \alpha \alpha\right)+\left(\nu \nu\left|f_{\sigma, \tau}^{\omega, x c}\left[\rho_{0}\right]\right| \beta \beta\right)\right] \\
\equiv & \frac{1}{4} S_{\mu \nu} S_{\alpha \beta}\left[\Gamma_{\mu \alpha}^{\sigma, \tau}+\Gamma_{\mu \beta}^{\sigma, \tau}+\Gamma_{\nu \alpha}^{\sigma, \tau}+\Gamma_{\nu \beta}^{\sigma, \tau}\right] .
\end{aligned}
$$

Os termos $\gamma$ são iguais aos introduzidos durante a derivação do formalismo independente do tempo (ver Subseção 2.4.3), e os com índice $l r$ alteram o kernel para a contribuição de longo alcance da decomposição de Yukawa. As integrais $(a a \mid b b)$ são então calculadas com auxílio das regras de SlaterKoster[35, 77] variando as distâncias internucleares para cada escolha do parâmetro de longo alcance (de 0.1 até $0.5 a_{0}^{-1}$ ). Como exemplo, um dos termos assume a forma,

$$
\gamma_{\alpha \beta}^{l r}=\gamma_{A B}^{l r}\left(R_{A B}\right)=\frac{\tau_{A}^{3} \tau_{B}^{3}}{(8 \pi)^{2}} \iint d \boldsymbol{r} d \boldsymbol{r}^{\prime} e^{-\tau_{A}\left\|\boldsymbol{r}-\boldsymbol{R}_{A}\right\|}\left(\frac{1-e^{-\omega\left\|\boldsymbol{r}-\boldsymbol{r}^{\prime}\right\|}}{\left\|\boldsymbol{r}-\boldsymbol{r}^{\prime}\right\|}\right) e^{-\tau_{B}\left\|\boldsymbol{r}^{\prime}-\boldsymbol{R}_{B}\right\|},
$$

onde $\alpha \in A, \beta \in B$ e $\tau_{i}$ é a constante de decaimento atômica, definida a partir da conexão entre $\gamma_{i i}$ e o parâmetro de Hubbard, ${ }^{16}$ dado por $(2.61)$. Seguindo a proposta de Domínguez [78], é conveniente fazer a transformação das densidades de spin $\alpha$ e $\beta,\left\{\rho_{\uparrow}, \rho_{\downarrow}\right\}$, para a densidade total e a magnetização, $\left\{\rho \equiv \rho_{\uparrow}+\rho_{\downarrow}, m \equiv \rho_{\uparrow}-\rho_{\downarrow}\right\}$, de tal forma que o kernel pode ser expresso como,

$$
f_{\sigma \tau}^{x c}=f^{x c}+\delta_{\sigma} \delta_{\tau} \tilde{f}^{x c}=\frac{\partial^{2} E_{x c}}{\partial \rho \partial \rho}+\delta_{\sigma} \delta_{\tau} \frac{\partial^{2} E_{x c}}{\partial m \partial m},
$$

onde $\delta_{\sigma}=2 \delta_{\sigma \uparrow}-1$. A partir dessa decomposição, o elemento de matriz do kernel pode ser separado em duas contribuições que envolvem apenas $\rho$ ou $m$. Expandindo em orbitais atômicos, $\mu=\{A l m\}$ e $\nu=\left\{B l^{\prime} m^{\prime}\right\}$ carregam as informações do momento angular $l$ e sua respectiva projeção $m$ dos orbitais $\mu$ e $\nu$ centrados nos átomos $A$ e $B$, respectivamente. E portanto,

\footnotetext{
${ }^{16}$ Nesse caso a relação não é tão direta quanto em (2.63), a expressão é dada pela equação C7 do Apêndice C do artigo [35].
} 


$$
f_{\mu \mu \sigma, \nu \nu \tau}^{\omega, x c}=\left(\mu \mu\left|f_{\sigma, \tau}^{\omega, x c}\left[\rho_{0}\right]\right| \nu \nu\right)=\Gamma_{\mu \nu}^{\sigma, \tau}=\gamma_{A l, B l^{\prime}}^{f r}+\delta_{\sigma} \delta_{\tau} \delta_{A B} W_{A l, l^{\prime}}
$$

O índice $f r$ diz respeito ao kernel full range que soma $f_{\sigma, \tau}^{\omega, x c}\left[\rho_{0}\right]$ com o termo de Hartree, $\left\|\boldsymbol{r}-\boldsymbol{r}^{\prime}\right\|^{-1}$. $W_{A l, l^{\prime}}$ são as constantes atômicas de spin, tratadas como parâmetros e otimizadas de maneira semelhante a $\gamma_{A l, B l^{\prime}}$ para cada conjunto de parâmetros de Slater-Koster. Finalmente, as equações de KS generalizadas para o formalismo LC-DFTB de camada aberta são,

$$
\sum_{\nu} H_{\mu \nu \sigma} c_{\nu i}^{\sigma}=\epsilon_{i \sigma} \sum_{\nu} S_{\mu \nu} c_{\nu i}^{\sigma}
$$

Admitindo que os orbitais moleculares são reais, os termos de acoplamento podem ser expressos como,

$$
K_{i a \sigma, i^{\prime} a^{\prime} \sigma^{\prime}}=\sum_{A, B} \sum_{l, l^{\prime}}\left(q_{A l}^{i a \sigma} \Gamma_{A l, B l^{\prime}}^{\sigma \sigma^{\prime}} q_{B l^{\prime}}^{i^{\prime} a^{\prime} \sigma^{\prime}}-\delta_{\sigma \sigma^{\prime}} q_{A l}^{i i^{\prime} \sigma} \gamma_{A l, B l^{\prime}}^{l r} q_{B l^{\prime}}^{a a^{\prime} \sigma^{\prime}}\right)
$$

onde $q_{A l}^{i a \sigma}$ é chamada carga de transição atômica,

$$
q_{A l}^{i a \sigma}=\frac{1}{2} \sum_{\mu \in A, l} \sum_{\nu}\left(c_{\mu i}^{\sigma} c_{\nu a}^{\sigma} S_{\mu \nu}+c_{\mu a}^{\sigma} c_{\nu i}^{\sigma} S_{\mu \nu}\right) .
$$

De posse da matriz de acoplamento $\mathbb{K}$, as matrizes $\mathbb{A}$ e $\mathbb{B}$ podem ser determinadas e portanto a equação (2.74) se reduz a um problema de autoestados e autovalores no qual o conjunto de autovalores $\{\Omega\}$ corresponde as energias de excitação, e as autofunções são associadas as forças de oscilador por meio da expressão,

$$
f=\frac{2 \Omega}{3} \sum_{k=1}^{3}\left|\sum_{i a \sigma}\left\langle\psi_{i \sigma}\left|\hat{\mathcal{R}}_{k}\right| \psi_{a \sigma}\right\rangle \sqrt{n_{i \sigma}-n_{a \sigma}}\left(X_{i a \sigma}+Y_{i a \sigma}\right)\right|^{2},
$$

onde $\hat{\mathcal{R}}_{k}$ denota a $k$-ésima componente do operador de posição ${ }^{17}$ e $n_{i \sigma}$ o número de ocupação do $i$-ésimo orbital com projeção de spin $\sigma$. Os dipolos de transição são tratados na aproximação de Mulliken,

$$
\left\langle\psi_{i \sigma}\left|\hat{\mathcal{R}}_{k}\right| \psi_{a \sigma}\right\rangle \simeq \sum_{A}\left(\boldsymbol{R}_{A}\right)_{k}\left(\sum_{l} q_{A l}^{i a \sigma}\right)
$$

\footnotetext{
${ }^{17}$ Aqui o operador de posição é entendido como a soma sobre todos os elétrons, de acordo com a equação (N.2).
} 
somando, sobre todos os átomos, o produto entre a carga de transição atômica total e a $k$-ésima coordenada de posição do respectivo átomo $\left(\left(\boldsymbol{R}_{A}\right)_{k}\right)$.

\subsection{Modelos de solvatação implícita}

A incorporação de solventes é de grande importância para a caracterização de diversos sistemas, especialmente no que diz respeito a compostos biológicos. No contexto do presente trabalho, os efeitos do solvente são investigados sobre os HPAs a fim de inferir propriedades do composto aceitador DTP-IC-4Ph, uma vez que Wang et al. fornecem picos de absorção do composto solvatado em solução de clorofórmio [15].

Os métodos que incorporaram tais efeitos são divididos em modelos de solvatação explícita e implícita. O primeiro consiste na descrição do solvente a partir de moléculas individuais, envolvendo uma abordagem estatística com simulações computacionais mais custosas. Já no modelo implícito, o solvente é representado por um meio contínuo onde os graus de liberdade das partículas constituintes são tipicamente retratados através de funções de distribuição [79]. Neste, a caracterização do sistema é dada por meio da construção de uma cavidade no interior de um meio dielétrico contínuo com as propriedades do solvente em questão. O soluto se encontra dentro da cavidade, e em nível eletrostático sua distribuição de carga $\rho_{M}$ polariza o dielétrico que por sua vez polariza a distribuição de carga do próprio soluto, formando um processo auto-consistente que leva a construção da chamada Self-Consistent Reaction Field Theory. O modelo básico parte da equação de Poisson,

$$
\nabla^{2} V(\boldsymbol{r})=\left\{\begin{array}{cc}
-4 \pi \rho_{M}(\boldsymbol{r}), & \text { dentro da cavidade } \\
0, & \text { fora da cavidade }
\end{array} .\right.
$$

O potencial é separado em duas partes,

$$
V(\boldsymbol{r})=V_{M}(\boldsymbol{r})+V_{P}(\boldsymbol{r})
$$

sendo $V_{M}$ a contribuição gerada pela densidade eletrônica $\rho_{M}$ e $V_{P}$ a parcela decorrente da polarização do dielétrico. As condições de contorno adotadas caracterizam vínculos físicos do problema e as soluções são tais que, 


$$
\begin{gathered}
{[V]=V_{\text {dentro }}-V_{\text {fora }}=0} \\
{[\partial V]=\left(\frac{\partial V}{\partial n}\right)_{\text {dentro }}-\epsilon\left(\frac{\partial V}{\partial n}\right)_{\text {fora }}=0,}
\end{gathered}
$$

onde $\epsilon$ é a constante dielétrica do solvente. Os potenciais devem ser contínuos em todo espaço, em particular na fronteira entre os meios, assim como suas derivadas ao longo da direção perpendicular à superfície da cavidade. No limite em que nos afastamos do soluto os campos convergem de maneira bem comportada,

$$
\begin{aligned}
& \lim _{r \rightarrow \infty} r V(r)=\alpha, \\
& \lim _{r \rightarrow \infty} r^{2} V(r)=\beta,
\end{aligned}
$$

onde $\alpha$ e $\beta$ são constantes finitas ${ }^{18}$. Uma vez estabelecidas as condições de contorno, a partir da densidade $\rho_{M}$ no vácuo é iniciado o procedimento auto-consistente cuja determinação das grandezas varia de acordo com o método em questão.

\subsubsection{Modelo contínuo polarizável (PCM)}

Ao longo dos cálculos DFT foi utilizado um dos modelos de solvatação implícita mais disseminados, o PCM. Sendo um dos métodos ASC ( $A p$ parent Surface Charge), a proposta consiste em descrever o potencial $V_{P}$ como o resultado de uma densidade de carga superficial ao longo da parede da cavidade,

$$
V_{P} \equiv V_{\sigma}(\boldsymbol{r})=\int_{\Gamma} d^{2} s \frac{\sigma(\boldsymbol{s})}{\|\boldsymbol{r}-\boldsymbol{s}\|} .
$$

A notação faz uso de $\boldsymbol{s}$ como variável espacial para enfatizar que a integral é ao longo da casca $\Gamma$ que estabelece a fronteira entre os meios, sendo $\sigma(\boldsymbol{s})$ a densidade superficial desta fronteira. Admitindo que o meio é linear ${ }^{19}$, o vetor de polarização de uma dada região do espaço é,

$$
\boldsymbol{P}_{i}(\boldsymbol{r})=-\frac{\epsilon_{i}-1}{4 \pi} \nabla V(\boldsymbol{r}),
$$

\footnotetext{
${ }^{18}$ Aplicando no contexto quântico as condições de convergência em questão garantem que a função de onda do sistema sujeito a $\mathcal{H}_{e f}(2.79)$ seja quadraticamente integrável.

${ }^{19}$ Nesse contexto subentende-se que o meio também é homogêneo, não dispersivo e isotrópico.
} 
sendo $\epsilon_{i}$ a constante dielétrica da região $i$. Na fronteira entre as regiões $i$ e $j$ a densidade de carga superficial aparente é dada por,

$$
\sigma_{i j}=-\left(\boldsymbol{P}_{j}-\boldsymbol{P}_{i}\right) \cdot \boldsymbol{n}_{i j},
$$

onde $\boldsymbol{n}_{i j}$ é o vetor normal a superfície que delimita as duas regiões, apontando do meio $i$ para o $j$ de tal forma que $\sigma(s)$ pode ser calculada por, ${ }^{20}$

$$
\sigma(\boldsymbol{s})=\frac{\epsilon-1}{4 \pi} \frac{\partial}{\partial \boldsymbol{n}}\left(V_{M}+V_{\sigma}\right)_{\text {dentro }}
$$

onde $\boldsymbol{n}$ é versor perpendicular a cavidade apontando de dentro para fora. Na prática, a superfície $\Gamma$ é então segmentada em pequenos elementos $\Delta S_{i}$ onde a densidade $\sigma\left(\boldsymbol{s}_{i}\right)$ é aproximadamente constante, e portanto a integral do potencial (2.77) pode ser expressa em termos de uma soma finita,

$$
V_{\sigma}(\boldsymbol{r}) \simeq \sum_{i} \frac{\sigma\left(\boldsymbol{s}_{i}\right) \Delta S_{i}}{\left\|\boldsymbol{r}-\boldsymbol{s}_{i}\right\|}=\sum_{i} \frac{q_{i}}{\left\|\boldsymbol{r}-\boldsymbol{s}_{i}\right\|} .
$$

No centro de cada região é atribuída uma carga pontual $q_{i}$ que passa por um primeiro processo iterativo. A densidade inicial $\sigma^{00}\left(\boldsymbol{s}_{i}\right)$ é obtida por meio de (2.78) sujeita a densidade do soluto no vácuo $\rho^{0}(\boldsymbol{r}) \equiv \rho_{M}(\boldsymbol{r})$. A carga pontual associada é então calculada por meio de,

$$
q_{i}^{00}=\sigma^{00}\left(\boldsymbol{s}_{i}\right) \Delta S_{i} .
$$

Essa carga gera uma nova contribuição no potencial $V_{\sigma}^{00}$ alterando a densidade superficial para $\sigma^{01}\left(\boldsymbol{s}_{i}\right)$ via $(2.78)$. Realizando esse procedimento ao longo de todas os elementos de superfície e iterando até satisfazer o critério de convergência, após um número $k$ de passos é gerada a nova densidade $\rho_{M}^{1}(\boldsymbol{r})$ a partir do conjunto de cargas $q_{i}^{0, k}$. Com a nova densidade, todas as etapas são repetidas até que para o $m$-ésimo passo os potenciais $V_{\sigma}^{m} \mathrm{e}$ $V_{\sigma}^{m-1}$ calculados a partir das densidades $\rho_{M}^{m}$ e $\rho_{M}^{m-1}$ convirjam. $^{21}$

Os efeitos do solvente são incorporados no formalismo quântico de maneira direta, a hamiltoniana do sistema passa a ser a hamiltoniana no vácuo $\mathcal{H}_{0}^{m}$, sujeita a densidade $\rho_{M}^{m}$ convergida com adição do potencial de reação do solvente $V_{\sigma}^{m}(\boldsymbol{r})$,

\footnotetext{
${ }^{20}$ Aqui o $\epsilon$ corresponde a constante dielétrica do meio, uma vez que no interior da cavidade a molécula se encontra no vácuo.

${ }^{21}$ Esse procedimento é esquematizado e detalhado do Apêndice J.
} 


$$
\mathcal{H}_{e f}=\mathcal{H}_{0}^{m}+V_{\sigma}^{m}(\boldsymbol{r})
$$

\subsection{Relação de Clausius-Mossotti}

Uma das grandezas físicas relevantes no estudo de células solares orgânicas é a constante dielétrica dos elementos que compõem a camada ativa do dispositivo fotovoltaico. Ainda que semicondutores orgânicos tradicionalmente apresentem pequenas constantes dielétricas $(\epsilon<6)$, valores elevados são de extrema importância pois diminuem a energia de ligação dos éxcitons e consequentemente a taxa de recombinação dessas quasipartículas, melhorando a performance da célula solar como um todo [26]. Ademais, computacionalmente é usual a utilização de modelos de solvatação implícita para caracterizar o efeito das demais moléculas, tanto no contexto de bulk como na descrição de polímeros. A camada ativa é composta por diversas unidades aceitadoras e doadoras, de tal forma que a caracterização do ambiente via solvente contribui significativamente para a qualidade dos resultados [80].

A determinação da constante dielétrica é realizada por meio de um resultado clássico conhecido como relação de Clausius-Mossotti, que surge tanto da interação de meios dielétricos quanto do estudo de índices de refração. A relação é dada pela equação, ${ }^{22}$

$$
\frac{\epsilon-1}{\epsilon-2}=\frac{4 \pi}{3}\left(\frac{\rho N_{A}}{M}\right) \alpha=\frac{4 \pi}{3} \frac{\alpha}{V} .
$$

onde $\rho$ é a densidade do material, $N_{A}$ é o número de Avogadro, $M$ a massa molar e $\alpha$ a polarizabilidade. Por meio da definição usual de densidade, a razão entre parênteses pode ser identificada como o inverso do volume de uma molécula $(V)$. A polarizabilidade total é entendida como uma soma de contribuições eletrônicas $\left(\alpha_{e}\right)$, iônicas $\left(\alpha_{i}\right)$ e orientacionais,

$$
\alpha=\alpha_{e}+\alpha_{i}+\frac{\mu^{2}}{3 k_{B} T}
$$

onde $\mu$ é o momento de dipolo permanente, $k_{B}$ a constante de Boltzmann e $T$ a temperatura. A primeira contribuição diz respeito à resposta do sistema frente a um campo elétrico, e.g. a interação com radiação ele-

\footnotetext{
${ }^{22}$ Por simplicidade, adotamos a notação simplificada $\epsilon \equiv \epsilon_{r}$.
} 
tromagnética. O termo iônico é fruto das interações entre os átomos que constituem o composto, sendo expressivo em sólidos cristalinos, e a contribuição orientacional é consequência do ordenamento natural do sistema, expresso em termos do momento de dipolo permanente, cujo efeito é reduzido a medida que a temperatura aumenta.

\subsection{Ensemble nuclear de configurações}

Uma das formas de incorporar efeitos vibracionais no espectro de absorção é realizada por meio da representação da função de onda vibracional a partir de um conjunto de configurações nucleares clássicas. Tal abordagem leva o nome de Nuclear Ensemble Approach e computa um observável físico $\mathcal{O}$ integrando seu valor ao longo de um conjunto representativo de coordenadas nucleares,

$$
\mathcal{O}=\int d \boldsymbol{x} f(\boldsymbol{x}) P(\boldsymbol{x}) \equiv E_{P}[f(\boldsymbol{X})] .
$$

A função $f(\boldsymbol{x})$ descreve o comportamento do observável no espaço de fases $\boldsymbol{x}=(\boldsymbol{q}, \boldsymbol{p})$, sendo $\boldsymbol{q}$ e $\boldsymbol{p}$ o conjunto de coordenadas e momentos nucleares, respectivamente. A distribuição de probabilidades normalizada é representada por $P(\boldsymbol{x})$ enquanto $E_{P}[\cdot]$ corresponde ao valor esperado do argumento sujeito a distribuição $P .{ }^{23}$ Do ponto de vista prático, a escolha da distribuição é arbitrária, mas, além de formalmente corretas, as distribuições quânticas tendem a apresentar resultados melhores. Uma vez que as vibrações são descritas a partir dos modos normais na aproximação de osciladores harmônicos, uma opção natural é a distribuição de Wigner pois possui expressão analítica [81],

$$
W(\boldsymbol{q}, \boldsymbol{p}, T)=\prod_{i=1}^{M}\left(\frac{\alpha_{i}}{\pi \hbar}\right) \exp \left(-\frac{\alpha_{i} \mu_{i} \omega_{i} q_{i}^{2}}{\hbar}-\frac{p_{i}^{2}}{\mu_{i} \omega_{i} \hbar}\right),
$$

onde $M$ é o número de modos vibracionais, $q_{i}$ a coordenada, $p_{i}$ o momento, $\mu_{i}$ a massa reduzida, e $\omega_{i}$ a frequência harmônica associados ao $i$-ésimo modo. A temperatura $T$ aparece por meio de $\alpha_{i}=\tanh \left(\frac{\hbar \omega_{i}}{2 k_{B} T}\right)$, onde $k_{B}$ é a constante de Boltzmann.

\footnotetext{
${ }^{23}$ A letra maiúscula $\boldsymbol{X}$ é utilizada para indicar que a variável esta sujeita a uma distribuição de probabilidades, nesse caso, $P$.
} 
No contexto de absorção de luz, visto que as transições entre estados acoplam via expansão multipolar, as probabilidades dependem apenas das coordenadas, e como a distribuição é separável não há termos mistos envolvendo $q_{i}$ e $p_{i}$. Desta forma, ao considerar $N_{c}$ configurações nucleares, o modelo utilizado para seção de choque de fotoabsorção em função da energia $E$ do fóton incidente é dado por [82],

$$
\sigma(E)=\frac{\pi e^{2} \hbar}{2 m_{e} c \epsilon_{0} N_{c} E} \sum_{i}^{N_{e s}} \sum_{j}^{N_{c}} \Omega_{0 i}\left(\boldsymbol{q}_{j}\right) f_{0 i}\left(\boldsymbol{q}_{j}\right) L\left(E-\Omega_{0 i}\left(\boldsymbol{q}_{j}\right), \delta\right) .
$$

A primeira somatória varre os $N_{e s}$ estados excitados, onde $\Omega_{0 i}\left(\boldsymbol{q}_{j}\right)$ e $f_{0 i}\left(\boldsymbol{q}_{j}\right)$ dizem respeito a energia de excitação do estado fundamental até o $i$-ésimo estado excitado na $j$-ésima configuração nuclear e a força de oscilador correspondente, respectivamente. A função $L$ representa o perfil fenomenológico, ${ }^{24}$ descrito através de uma lorentziana centrada em $\Omega_{0 i}$ e com uma largura $\delta$ relacionada ao tempo de vida do estado excitado frente ao decaimento radiativo.

\footnotetext{
${ }^{24}$ Outros perfis podem ser ajustados, e.g., uma gaussiana, porém a lorentziana é a solução de equações diferenciais do oscilador harmônico amortecido e de problemas de espalhamento, sendo portanto a escolha mais natural.
} 


\section{Capítulo 3}

\section{Resultados}

Nessa seção serão apresentados os resultados obtidos ao longo do projeto, que envolvem a determinação de propriedades óticas de sistemas moleculares com tamanho considerável. Para tal, a metodologia LC-(TD)DFTB/OB2+S é utilizada, e por meio do estudo dos HPAs busca-se validar o modelo semi-empírico frente ao modelo (TD)-DFT/ $\omega$ B97X-D/6$31 \mathrm{G}(\mathrm{d}, \mathrm{p})$, frequentemente utilizado na caracterização de moléculas que compõem a camada ativa de células solares [54, 55, 21, 22, 56, 57, 58]. A sensibilidade diante da base atômica é explorada, buscando elucidar o que podemos esperar de um tratamento mais sofisticado, e o impacto do meio dielétrico é investigado a partir do modelo de solvatação PCM [79].

A escolha do parâmetro de longo alcance $\omega$ visa minimizar o erro de autointeração [83] e é realizada, em ambos os modelos, via imposição do Teorema de Janak, como usual na LC-DFT $[84,85]$. De posse dos parâmetros ótimos, os espectros de absorção são determinados assim como suas médias sobre a região de Franck-Condon. O levantamento dos espectros do aceitador eletrônico DTP-IC-4Ph e do doador eletrônico PBDB-T compõem o objetivo central do projeto e são comparados com resultados experimentais. Por fim, são determinadas grandezas importantes para a modelagem dos dispositivos fotovoltaicos, i.e., constantes dielétricas, energias de ligação dos éxcitons e energias intramoleculares de reorganização.

\subsection{Efeito das funções de base na estrutura eletrônica}

Os compostos $\pi$-conjugados são caracterizados por anéis de benzeno com suas estruturas ressonantes rígidas, e também pelos orbitais moleculares com energias próximas ao HOMO serem localizados com caráter $\pi / \pi^{*}$. A priori, não há demanda pela utilização de funções atômicas difusas ou de polarização para a caracterização do estado fundamental do composto. Entretanto, os estados excitados que são descritos em termos de excitações entre orbitais com energias fora da região discutida, podem acarretar em 
maior sensibilidade do espectro de absorção com relação à base atômica.

Como a metodologia DFTB envolve o cálculo prévio dos elementos de matriz, não há liberdade frente a escolha do funcional de correlação e troca e nem da base atômica. A fim de elucidar possíveis limitações dada a utilização da base mínima de funções de Slater na DFTB, foram realizados cálculos DFT para os HPAs com diferentes bases atômicas visando a comparação com os resultados em nível DFTB.

Em todos os cálculos realizados nessa seção o nível de teoria utilizado foi (TD)-DFT/ $\omega$ B97X-D com diferentes bases atômicas e o parâmetro de longo alcance fixado em $\omega=0.2 a_{0}^{-1}$, valor esse obtido por meio do procedimento de otimização que é explanado na Seção 3.2.

\subsubsection{Geometria}

A abordagem utilizada para comparar os resultados das otimizações de geometria consiste em explorar a raiz do desvio quadrático médio (RMSD), definida como,

$$
R M S D=\sqrt{\frac{\sum_{i}^{N}\left(\boldsymbol{r}_{i}^{(1)}-\boldsymbol{r}_{i}^{(2)}\right)^{2}}{N}},
$$

onde $\boldsymbol{r}_{i}^{(n)}$ diz respeito ao vetor posição do $i$-ésimo átomo, $N$ é o número de átomos, e os índices superiores (1) e (2) fazem referência a duas bases atômicas distintas. Cada um dos átomos é enumerado e são comparadas as respectivas posições nas geometrias otimizadas utilizando o mesmo funcional $\omega \mathrm{B} 97 \mathrm{X}-\mathrm{D}$ e duas bases atômicas distintas, de tal forma que quanto maior o valor absoluto, maior a diferença entre as geometrias do composto. Essa medida traz a noção de desvio médio das coordenadas nucleares, carregando informações a respeito de distâncias interatômicas, ângulos de ligação e torsão, assim como efeitos de tautomerização. Para evitar erros decorrentes de deslocamentos rígidos, o algoritmo implementado no software Chemcraft [86] rotaciona e translada os compostos de forma a minimizar o RMSD, de modo que o erro associado à rotações rígidas é da ordem de $10^{-14} \AA$ para moléculas com 50 à 100 átomos. A Tabela 3.1 apresenta, para as moléculas de naftaleno e pireno, a comparação entre as diferenças bases atômicas tomando como referência os resultados obtidos 
com a base $6-31 G(d, p)$.

\begin{tabular}{c|cc} 
& \multicolumn{2}{|c}{ RMSD $(\AA)$} \\
\hline Base atômica & Naftaleno & Pireno \\
\hline STO-3G & 0.0256 & 0.0332 \\
6-31G & 0.0055 & 0.0064 \\
6-311G $(\mathrm{d}, \mathrm{p})$ & 0.0040 & 0.0043 \\
6-311G++(d,p) & 0.0032 & 0.0036 \\
cc-pVDZ & 0.0068 & 0.0071 \\
aug-cc-pVDZ & 0.0060 & 0.0062 \\
aug-cc-pVTZ & 0.0094 & 0.0115 \\
\hline Média & 0.0075 & 0.0090 \\
DAM & 0.0050 & 0.0067 \\
\hline
\end{tabular}

Tabela 3.1: Raízes do desvio quadrático médio (RMSD) dos comprimentos de ligação em relação a geometria otimizada com a base atômica $6-31 \mathrm{G}(\mathrm{d}, \mathrm{p})$ para as moléculas de naftaleno e pireno. As duas últimas linhas dizem respeito a média e o desvio absoluto médio.

A principal diferença observada é com relação à base mínima de funções do tipo Slater, que apresenta variações da ordem de $10^{-2} \AA$, e, por ser uma base pequena, exibe resultados com qualidade inferior em relação as demais. Todavia, as variações médias na ordem de $10^{-3} \AA$ indicam que no geral a base atômica não possui grande impacto na geometria dos HPAs.

\subsubsection{Estado fundamental e orbitais moleculares}

Uma vez conhecida a sensibilidade da geometria do estado fundamental frente à escolha da base atômica, passamos a estudar o efeito da base sobre a estrutura eletrônica. As Tabelas 3.2 e 3.3 trazem os valores de energia do estado fundamental, energia dos orbitais HOMO e LUMO, assim como sua diferença $\left(E_{\text {gap }}^{H-L}\right)$, para os compostos de naftaleno e pireno, respectivamente. 


\begin{tabular}{c|cccc} 
Base atômica & Energia $(\mathrm{H})$ & $\epsilon_{\text {Номо }}(\mathrm{eV})$ & $\epsilon_{L U M O}(\mathrm{eV})$ & $E_{\text {gap }}^{H-L}(\mathrm{eV})$ \\
\hline STO-3G & -381.102 & -6.251 & 3.166 & 9.417 \\
6-31G & -385.672 & -7.768 & 0.884 & 8.652 \\
6-31G(d,p) & -385.767 & -7.748 & 0.830 & 8.578 \\
6-311G(d,p) & -385.840 & -7.952 & 0.564 & 8.516 \\
6-311++G(d,p) & -385.843 & -8.015 & 0.432 & 8.448 \\
cc-pVDZ & -385.781 & -7.895 & 0.638 & 8.533 \\
aug-cc-pVDZ & -385.794 & -7.997 & 0.437 & 8.433 \\
aug-cc-pVTZ & -385.880 & -7.989 & 0.443 & 8.432 \\
\hline Média & -385.797 & -7.909 & 0.604 & 8.513 \\
DAM & 0.049 & 0.090 & 0.154 & 0.065 \\
\hline
\end{tabular}

Tabela 3.2: Energias do estado fundamental, dos orbitais HOMO $\left(\epsilon_{\text {Hомо }}\right)$ e LUMO $\left(\epsilon_{L U M O}\right)$ assim como suas diferenças $E_{\text {gap }}^{H-L}$, calculadas para a molécula de naftaleno utilizando o funcional $\omega$ B97X-D e variando a base atômica. As duas últimas linhas dizem respeito a média e o desvio absoluto médio desconsiderando a base STO-3G.

\begin{tabular}{c|cccc} 
Base atômica & Energia $(\mathrm{H})$ & $\epsilon_{\text {номо }}(\mathrm{eV})$ & $\epsilon_{\text {LU⿻ }}(\mathrm{eV})$ & $E_{\text {gap }}^{H-L}(\mathrm{eV})$ \\
\hline STO-3G & -608.132 & -5.546 & 2.326 & 7.872 \\
6-31G & -615.423 & -7.140 & 0.196 & 7.336 \\
6-31G(d,p) & -615.572 & -7.131 & 0.158 & 7.289 \\
6-311G(d,p) & -615.685 & -7.338 & -0.081 & 7.257 \\
6-311G++(d,p) & -615.689 & -7.394 & -0.172 & 7.222 \\
cc-pVDZ & -615.598 & -7.283 & -0.015 & 7.268 \\
aug-cc-pVDZ & -615.618 & -7.374 & -0.155 & 7.219 \\
aug-cc-pVTZ & -615.749 & -7.364 & -0.146 & 7.218 \\
\hline Média & -615.619 & -7.289 & -0.031 & 7.258 \\
DAM & 0.076 & 0.090 & 0.123 & 0.034 \\
\hline
\end{tabular}

Tabela 3.3: Energias do estado fundamental, dos orbitais HOMO $\left(\epsilon_{\text {Номо }}\right)$ e LUMO $\left(\epsilon_{L U M O}\right)$ assim como suas diferenças $E_{\text {gap }}^{H-L}$, calculadas para a molécula de pireno utilizando o funcional $\omega \mathrm{B} 97 \mathrm{X}-\mathrm{D}$ e variando a base atômica. As duas últimas linhas dizem respeito a média e o desvio absoluto médio desconsiderando a base STO-3G.

Com exceção da base STO-3G, a energia do estado fundamental não sofreu variações significativas, todas na ordem de 0.1 Hartree, tanto com relação à 
média quanto com relação à 6-31G(d,p). As energias dos orbitais também apresentam comportamento semelhante, com variações em torno de $0.1 \mathrm{eV}$ que tendem a estabilizar o gap HOMO-LUMO. A Figura 3.1 ilustra as isosuperfícies dos orbitais HOMO e LUMO da molécula de pireno obtidas com diferentes bases atômicas.

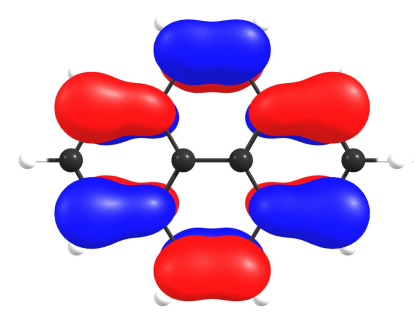

(a) (H) STO-3G

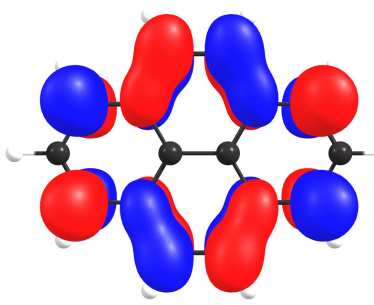

(e) (L) STO-3G

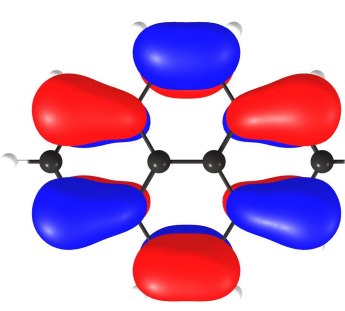

(b) (H) 6-31G(d,p)

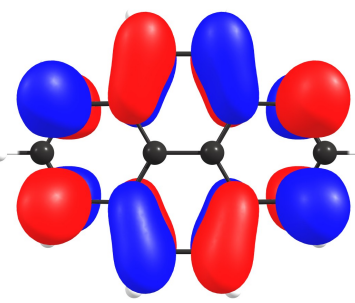

(f) (L) 6-31G(d,p)

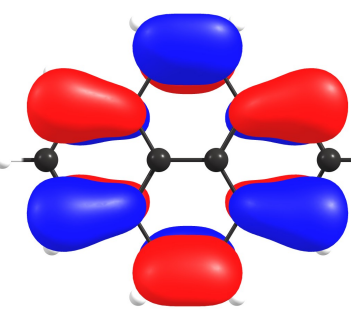

(c) $(\mathrm{H}) 6-311++\mathrm{G}(\mathrm{d}, \mathrm{p})$

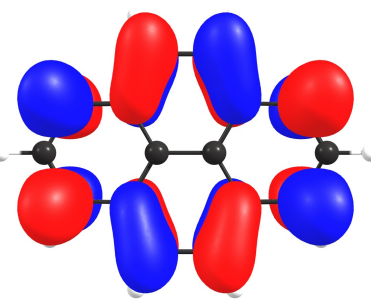

(g) (L) 6-311++G(d,p)

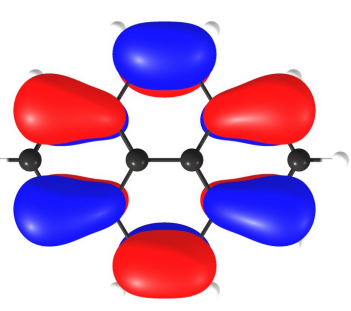

(d) (H) aug-cc-pVTZ

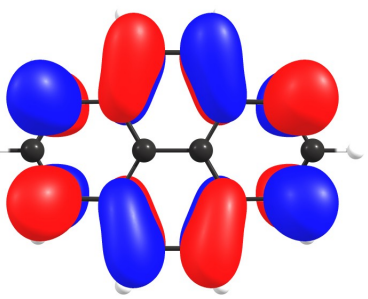

(h) (L) aug-cc-pVTZ

Figura 3.1: Isosuperfícies dos orbitais HOMO (primeira linha) e LUMO (segunda linha) da molécula de pireno para diferentes bases atômicas. O módulo do isovalor é fixado em 0.3 .

A inspeção dos orbitais calculados com cada base não indica diferenças significativas, as distribuições são essencialmente invariantes, preservando as probabilidades. À parte de eventuais inversões nas fases, tal comportamento também é observado para os orbitais $\mathrm{HOMO}+\mathrm{X}$ e $\mathrm{LUMO}+1$, onde $\mathrm{X}$ varia de 1 até 5 , o que mantém a compatibilidade de propriedades relacionadas. O aumento no número de funções de base resulta em novas combinações lineares e portanto aumenta o número de orbitais moleculares, o que acaba por alterar o ordenamento energético dos mesmos a partir dos orbitais $\mathrm{LUMO}+2$, e.g., o orbital $\mathrm{LUMO}+2$ da STO-3G passa a ser o $\mathrm{LUMO}+4$ da $6-311++\mathrm{G}(\mathrm{d}, \mathrm{p})$, o LUMO +5 da $6-311++\mathrm{G}(\mathrm{d}, \mathrm{p})$ passa a ser o LUMO+7 da aug-cc-pVTZ, etc. Um último ponto diz respeito à baixa compatibilidade entre o ordenamento dos orbitais da $6-311++\mathrm{G}(\mathrm{d}, \mathrm{p})$ e os da aug-cc-pVTZ, em oposição à alta compatibilidade da base de Pople com relação à aug-cc-pVDZ. Esse comportamento sugere que o número de primitivas tem maior impacto nas energias do que o número de con- 
trações, visto que a $6-311++\mathrm{G}(\mathrm{d}, \mathrm{p})$ é triple-zeta e possui $(9,36)^{1}$ primitivas, enquanto a aug-cc-pVDZ é double-zeta com $(9,23)$ primitivas e a aug-cc-pVTZ é triple-zeta com $(23,46)$ primitivas.

\subsubsection{Estados excitados e espectros de absorção}

No que diz respeito aos estados excitados, as Tabelas 3.4 e 3.5 fornecem, para os compostos de naftaleno e pireno, as energias de transição, e respectivas forças de oscilador, relativas às excitações do estado fundamental $\left(S_{0}\right)$ para o primeiro estado excitado $\left(S_{1}\right)$ e para o primeiro estado oticamente ativo $\left(S_{B}\right)$.

\begin{tabular}{c|cccc} 
Base atômica & $S_{0} \rightarrow S_{1}(\mathrm{eV})$ & $f_{1}$ & $S_{0} \rightarrow S_{B}(\mathrm{eV})$ & $f_{B}$ \\
\hline STO-3G & 5.520 & 0.0018 & 7.517 & 1.419 \\
6-31G & 4.825 & 0.0004 & 6.462 & 1.319 \\
6-31G(d,p) & 4.736 & 0.0004 & 6.330 & 1.270 \\
6-311G(d,p) & 4.682 & 0.0001 & 6.234 & 1.300 \\
6-311G++(d,p) & 4.646 & 0.0000 & 6.095 & 1.326 \\
cc-pVDZ & 4.696 & 0.0002 & 6.266 & 1.284 \\
aug-cc-pVDZ & 4.633 & 0.0000 & 6.072 & 1.335 \\
aug-cc-pVTZ & 4.627 & 0.0000 & 6.064 & 1.327 \\
\hline Média & 4.692 & 0.0002 & 6.218 & 1.309 \\
DAM & 0.052 & 0.0002 & 0.121 & 0.021 \\
\hline
\end{tabular}

Tabela 3.4: Energias de transição, e respectivas forças de oscilador $(f)$, do primeiro estado excitado e do primeiro estado brilhante da molécula de naftaleno. Na geometria otimizada via $\omega \mathrm{B} 97 \mathrm{X}-\mathrm{D} / 6-31 \mathrm{G}(\mathrm{d}, \mathrm{p})$ os estados excitados foram calculados variando a base atômica. As duas últimas linhas dizem respeito a média e o desvio absoluto médio desconsiderando a STO-3G.

\footnotetext{
${ }^{1} \mathrm{O}$ par ordenado $(X, Y)$ denota que são $X$ primitivas para hidrogênio e hélio, e $Y$ primitivas para átomos na faixa Li-Ne.
} 


\begin{tabular}{c|cccc} 
Base atômica & $S_{0} \rightarrow S_{1}(\mathrm{eV})$ & $f_{1}$ & $S_{0} \rightarrow S_{B}(\mathrm{eV})$ & $f_{B}$ \\
\hline STO-3G & 4.733 & 0.0011 & 6.972 & 1.166 \\
6-31G & 4.152 & 0.0000 & 6.009 & 1.033 \\
6-31G(d,p) & 4.070 & 0.0000 & 5.851 & 0.998 \\
6-311G(d,p) & 4.027 & 0.0002 & 5.763 & 1.010 \\
6-311G++(d,p) & 4.005 & 0.0003 & 5.687 & 1.047 \\
cc-pVDZ & 4.037 & 0.0001 & 5.786 & 1.007 \\
aug-cc-pVDZ & 3.991 & 0.0003 & 5.665 & 1.051 \\
aug-cc-pVTZ & 3.986 & 0.0003 & 5.656 & 1.049 \\
\hline Média & 4.038 & 0.0002 & 5.774 & 1.028 \\
DAM & 0.043 & 0.0001 & 0.093 & 0.020 \\
\hline
\end{tabular}

Tabela 3.5: Energias de transição, e respectivas forças de oscilador $(f)$, do primeiro estado excitado e do primeiro estado brilhante da molécula de pireno. Na geometria otimizada via $\omega \mathrm{B} 97 \mathrm{X}-\mathrm{D} / 6-31 \mathrm{G}(\mathrm{d}, \mathrm{p})$ os estados excitados foram calculados variando a base atômica. As duas últimas linhas dizem respeito a média e o desvio absoluto médio desconsiderando a STO-3G.

Novamente a STO-3G diverge em todas as grandezas, enquanto observamos maior convergência entre as demais bases. As variações na ordem de $0.1 \mathrm{eV}(\approx 2 \mathrm{kcal} / \mathrm{mol})$ são pequenas para ambas as energias de transição, que apresentam redução nos valores absolutos em decorrência essencialmente do aumento no tamanho da base. Ainda que suave, as variações observadas para a transição $S_{0} \rightarrow S_{B}$ são sistematicamente maiores, o que pode ser atribuído ao caráter mais delocalizado dos orbitais moleculares que compõem as transições de estados excitados de maior energia, salientando a necessidade de funções de base com caráter difuso. Inspecionando as forças de oscilador verificamos que as diferenças são ainda menores, o que minimiza o impacto na absorbância dos compostos.

As tabelas ilustram, em baixas energias, o comportamento dos espectros de absorção apresentados nas Figuras 3.2 e 3.3. Os espectros são, respectivamente, dos compostos de naftaleno e pireno, e foram obtidos a partir do ajuste de lorentzianas centradas nas energias de excitação de 50 estados excitados. A determinação das curvas é discutida com mais detalhe na Seção 3.4. 


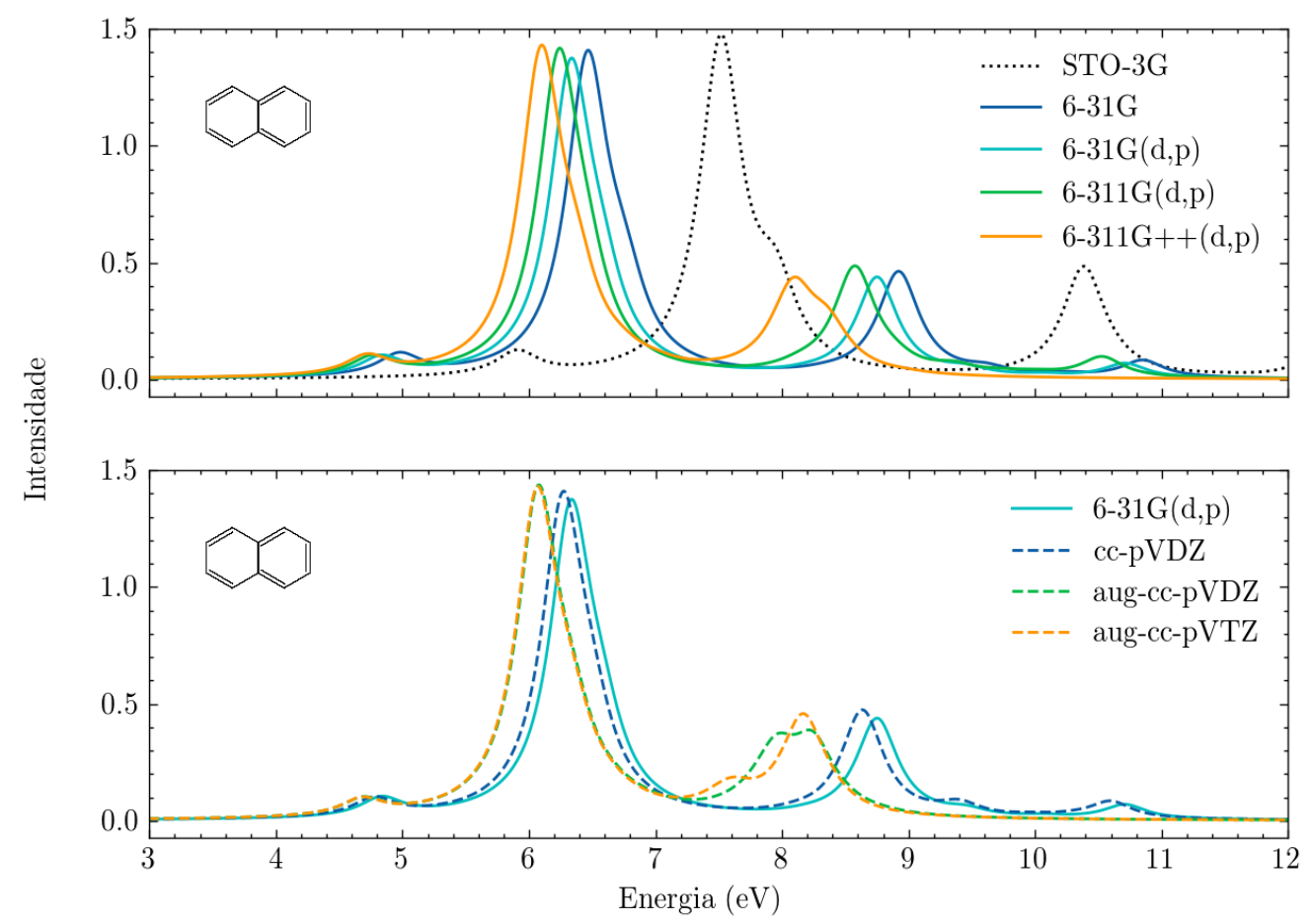

Figura 3.2: Espectros de absorção da molécula de naftaleno, obtidos variando a base atômica na geometria otimizada com a base 6-31G(d,p). No painel de cima são apresentados os resultados para as funções de Pople e no debaixo para as funções da família cc (correlated-consistent). Foram considerados 50 estados excitados em cada cálculo.

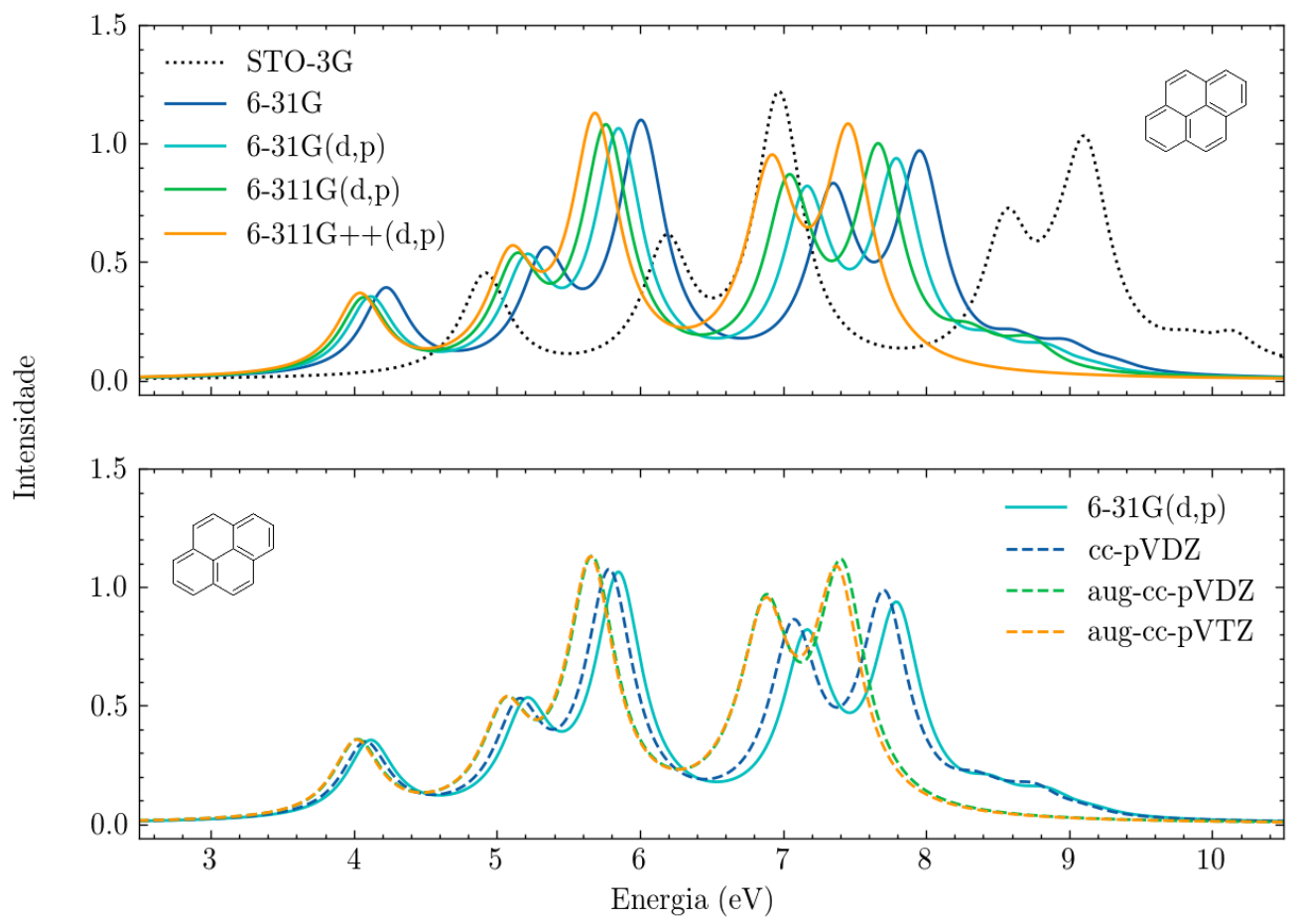

Figura 3.3: Espectros de absorção da molécula de pireno, obtidos variando a base atômica na geometria otimizada com a base $6-31 \mathrm{G}(\mathrm{d}, \mathrm{p})$. No painel de cima são apresentados os resultados para as funções de Pople e no debaixo para as funções da família cc (correlatedconsistent). Foram considerados 50 estados excitados em cada cálculo. 
Em primeira aproximação, para ambos os compostos observamos um deslocamento rígido do espectro para esquerda (red shift) conforme aumentamos o tamanho da base atômica, mantendo a largura do pico de maior intensidade essencialmente preservada. O mesmo efeito está presente nos demais picos da molécula de pireno, e ao comparar os resultados obtidos com as bases aug-cc-pVDZ e aug-cc-pVTZ, fica evidente que a mudança de duplo para triplo zeta e o aumento no número de funções de polarização praticamente não altera o espectro de absorção e portanto, no que diz respeito as propriedades de interesse, não justifica o acréscimo no custo computacional. Para o composto de naftaleno, o pico em torno de 8 eV não apresenta o mesmo comportamento dos demais. A adição de funções de polarização, em ambas as famílias de funções de base, tende a deslocar e alargar o pico. Já o aumento no número de funções difusas tende a adensar o espectro (maior número de estados por intervalo de energia), alterando a forma do pico e acabando por impactar na largura do mesmo.

\subsection{Otimização do parâmetro de longo alcance}

Uma vez que os termos de troca GGA e meta-GGA $\left(E_{x}^{G G A / m G G A}\right)$ são tipicamente obtidos apenas a partir da densidade eletrônica, os cálculos envolvem integrais de $u m$ elétron, ${ }^{2}$ em oposição às integrais de dois elétrons do termo de troca exato presente no método de Hartree-Fock. Desta forma, é razoável considerar (ainda que em parte) a contribuição exata $E_{x}^{H F}$ em funcionais de correlação e troca como meio de incorporar efeitos não-locais na energia. Outra forma de contornar tais limitações é introduzir a correção de longo alcance. No funcional $\omega \mathrm{B} 97 \mathrm{X}-\mathrm{D}$ essas correções são incorporadas através da decomposição (2.23), enquanto a metodologia DFTB implementada no software DFTB+ utiliza da decomposição análoga de Yukawa (2.68), buscando corrigir o comportamento assintótico da interação de longo alcance que é usualmente mal descrito por funcionais convencionais [63].

A escolha do parâmetro de longo alcance $\omega$ é a priori livre, podendo ser otimizada para cada sistema de interesse. Em ambas as metodologias DFT e DFTB, o valor de $\omega$ foi obtido de acordo com a proposta de Stein et al. [84, 85] que utiliza do Teorema de Janak [87] como fundamento. Uma consequência direta do teorema é que, em módulo, a energia do HOMO

\footnotetext{
${ }^{2}$ Como exemplo temos os funcionais B 97 [52] e BNL $[73,74]$, utilizados no presente trabalho.
} 
deve ser igual ao potencial de ionização, e a energia do LUMO igual à afinidade eletrônica [32]. Desta forma, foram adotados dois critérios, que consistem em escolher $\omega$ tal que a soma,

$$
J_{1} \equiv\left|I P+\epsilon_{H O M O}\right|=\left|E_{N-1}-E_{N}+\epsilon_{H O M O}\right| .
$$

e de maneira mais geral,

$$
\begin{aligned}
J_{3} & \equiv\left|I P+\epsilon_{H O M O}\right|+\left|E A+\epsilon_{L U M O}\right| \\
& =\left|E_{N-1}-E_{N}+\epsilon_{H O M O}\right|+\left|E_{N}-E_{N+1}+\epsilon_{L U M O}\right|,
\end{aligned}
$$

possuam o módulo tão próximo a zero quanto possível. As energias dos orbitais HOMO e LUMO são extraídas do cálculo do composto neutro enquanto o potencial de ionização $(I P)$ corresponde à energia vertical de ionização, determinada a partir da subtração da energia do cátion na geometria do neutro $\left(E_{N-1}\right)$ pela energia do estado fundamental do composto neutro $\left(E_{N}\right)$, enquanto a afinidade eletrônica $(E A)$ corresponde à subtração entre energia do estado fundamental do neutro $\left(E_{N}\right)$ e energia do ânion na geometria otimizada para o neutro $\left(E_{N+1}\right)$.

\subsubsection{Hidrocarbonetos poli-aromáticos}

Os HPAs estudados são apresentados na Figura 3.4 e suas estruturas compõem o esqueleto dos compostos aceitadores introduzidos por Wang et al. [15].

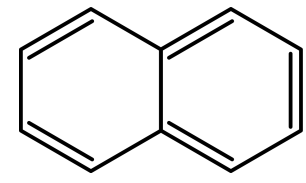

(a) Naftaleno $\left(\mathrm{C}_{10} \mathrm{H}_{8}\right)$

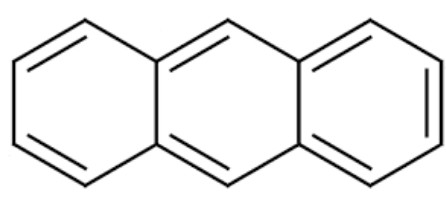

(b) Antraceno $\left(\mathrm{C}_{14} \mathrm{H}_{10}\right)$

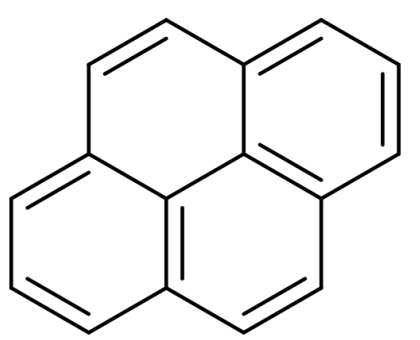

(c) Pireno $\left(\mathrm{C}_{16} \mathrm{H}_{10}\right)$

Figura 3.4: Representação dos hidrocarbonetos poliaromáticos estudados no projeto.

Em nível DFT, os cálculos de otimização de geometria das moléculas neutras assim como a determinação das energias dos íons, foram realizados utilizando o funcional $\omega$ B97X-D com a base atômica $6-31 \mathrm{G}(\mathrm{d}, \mathrm{p})$ através do pacote computacional Gaussian09 [88]. No formalismo DFTB, o software utilizado foi o DFTB+ [89] juntamente a uma versão adaptada do 
conjunto OB2 (Parametrization for Organic and Biological Molecules) de parâmetros de Slater-Koster desenvolvida pelos pesquisadores Dr. Van Quan Vuong e Prof. Stephan Irie (Oak Ridge National Laboratory, EUA) ${ }^{3}$, que leva em conta o átomo de enxofre $(\mathrm{OB} 2+\mathrm{S})$. Correções de dispersão no modelo de Slater-Kirkwood também foram consideradas (ver Apêndice $\mathrm{K})$.

A escolha do parâmetro de longo alcance é limitada no caso da DFTB pois são disponibilizados apenas os valores $0.1,0.2,0.3,0.4$ e $0.5 a_{0}^{-1}$. Cada $\omega$ demanda que os parâmetros de Slater-Koster sejam recalculados, passando pelo procedimento custoso de integração numérica dos elementos de matriz [70]. Com a intenção de simplificar o texto, as siglas em itálico $D F T$ e DFTB passam a fazer referência aos métodos (TD)-DFT/ $\omega$ B97X$\mathrm{D} / 6-31 \mathrm{G}(\mathrm{d}, \mathrm{p})$ e LC(-TD)-DFTB/OB2+S, respectivamente. Referências às metodologias DFT e DFTB permanecem sem itálico. Tendo em vista essa nomenclatura, os valores dos critérios de otimização do parâmetro de longo alcance para a molécula de naftaleno são apresentados na tabela abaixo.

\begin{tabular}{c|cc|cc} 
& \multicolumn{2}{|c|}{$\boldsymbol{D F T}$} & \multicolumn{2}{c}{ DF TB } \\
\hline$\omega\left(a_{0}^{-1}\right)$ & $J_{1}(\mathrm{eV})$ & $J_{3}(\mathrm{eV})$ & $J_{1}(\mathrm{eV})$ & $J_{3}(\mathrm{eV})$ \\
\hline 0.10 & 1.901 & 3.552 & 1.504 & 3.008 \\
0.18 & 0.062 & 0.096 & - & - \\
0.20 & 0.135 & 0.250 & 0.919 & 1.838 \\
0.30 & 0.424 & 0.624 & 0.560 & 1.120 \\
0.40 & 0.322 & 0.346 & 0.327 & 0.655 \\
0.50 & 0.253 & 0.398 & 0.169 & 0.340 \\
\hline
\end{tabular}

Tabela 3.6: Critérios de otimização $J_{1}$ e $J_{3}$ calculados para a o molécula de naftaleno variando o parâmetro de longo alcance $(\omega)$ no intervalo de 0.1 até $0.5 a_{0}^{-1}$.

Na $D F T$ é possível variar o parâmetro continuamente, e os valores ótimos obtidos foram respectivamente $0.18,0.20$ e $0.20 a_{0}^{-1}$ para as moléculas de naftaleno, antraceno e pireno. Tendo em vista as pequenas flutuações nos critérios $J_{i}$ e a proximidade entre os HPAs, o valor de $\omega$ dos HPAs foi fixado como $0.2 a_{0}^{-1}$ para a DFT. Para a DFTB o comportamento decrescente dos critérios $\left(J_{1}\right.$ e $\left.J_{3}\right)$ não caracteriza um ponto de mínimo, mas dadas

\footnotetext{
${ }^{3}$ Até o presente momento a parametrização não tornou-se pública mas foi utilizada em trabalho recente que investiga o impacto da distribuição espacial de doadores e aceitadores na formação de éxcitons [23].
} 
as limitações o valor mais adequado é de $0.5 a_{0}^{-1}$. Essa diferença entre os parâmetros ótimos obtidos para $D F T$ e $D F T B$ não é inusitada, uma vez que além de estarmos diante de formalismos diferentes, são utilizados funcionais e conjuntos de bases atômicas distintos em cada metodologia, não havendo razão para esperar que o valor de $\omega$ seja o mesmo nos dois casos. A mesma discrepância pode ser observada na Tabela 3.7 que traz os parâmetros otimizados ${ }^{4}$ e respectivos critérios, obtidos para os demais hidrocarbonetos poli-aromáticos.

\begin{tabular}{c|ccc|ccc} 
& \multicolumn{3}{|c|}{ DF T } & \multicolumn{3}{c}{ DF TB } \\
\hline Sistema & $\omega\left(a_{0}^{-1}\right)$ & $J_{1}(\mathrm{eV})$ & $J_{3}(\mathrm{eV})$ & $\omega\left(a_{0}^{-1}\right)$ & $J_{1}(\mathrm{eV})$ & $J_{3}(\mathrm{eV})$ \\
\hline \multirow{2}{*}{ Naftaleno } & 0.18 & 0.062 & 0.096 & 0.50 & 0.170 & 0.340 \\
& 0.20 & 0.135 & 0.250 & & & \\
Antraceno & 0.20 & 0.010 & 0.016 & 0.50 & 0.035 & 0.074 \\
Pireno & 0.20 & 0.014 & 0.022 & 0.50 & 0.034 & 0.078 \\
\hline
\end{tabular}

Tabela 3.7: Parâmetros de longo alcance otimizados e respectivos valores de $J_{1}$ e $J_{3}$ para os compostos de naftaleno, antraceno e pireno, usando ambas as metodologias.

Também foram utilizados valores experimentais do potencial de ionização e afinidade eletrônica para calibrar o parâmetro de longo alcance. É importante notar que ao utilizar dados experimentais, o valor otimizado de $\omega$ busca corrigir limitações do método como um todo, associadas ao funcional de correlação e troca, à base atômica e até mesmo às aproximações da metodologia, e.g., a expansão da energia até a segunda ordem em torno da densidade de átomos livres na DFTB, discutida na Subseção 2.4. As Figuras 3.5 e 3.6 apresentam o comportamento dos critérios de otimização $J_{1}$ e $J_{3}$ em função de $\omega$.

\footnotetext{
${ }^{4}$ Ainda que a otimização seja parcial, entende-se que o parâmetro é ótimo dentre aqueles disponíveis.
} 

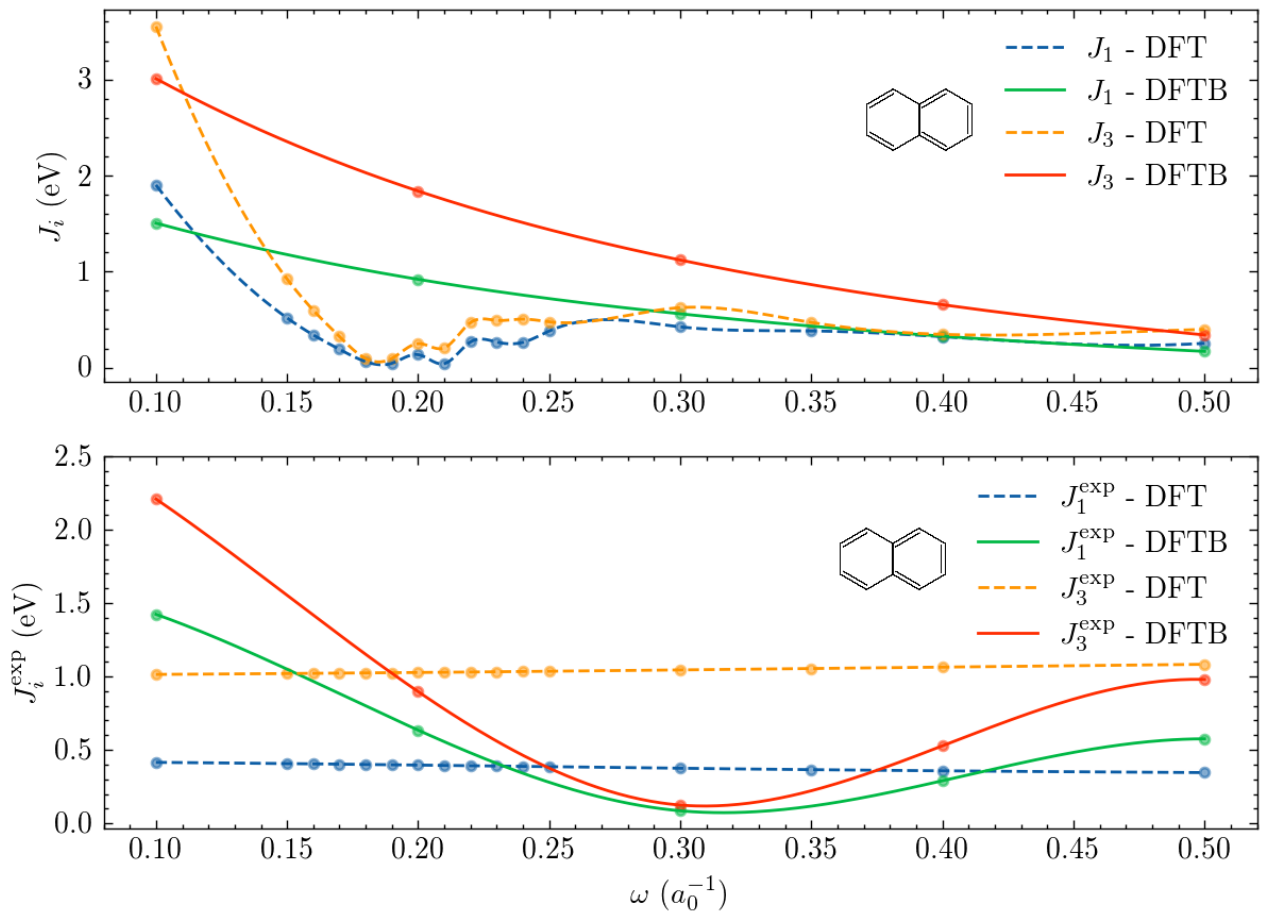

Figura 3.5: Critérios de otimização $J_{1}$ e $J_{3}$ em função do parâmetro de longo alcance $(\omega)$ para a molécula de naftaleno. No painel superior os valores foram obtidos utilizando o potencial de ionização $(I P)$ e a afinidade eletrônica $(E A)$ calculados, enquanto no painel inferior foram utilizados os valores experimentais.
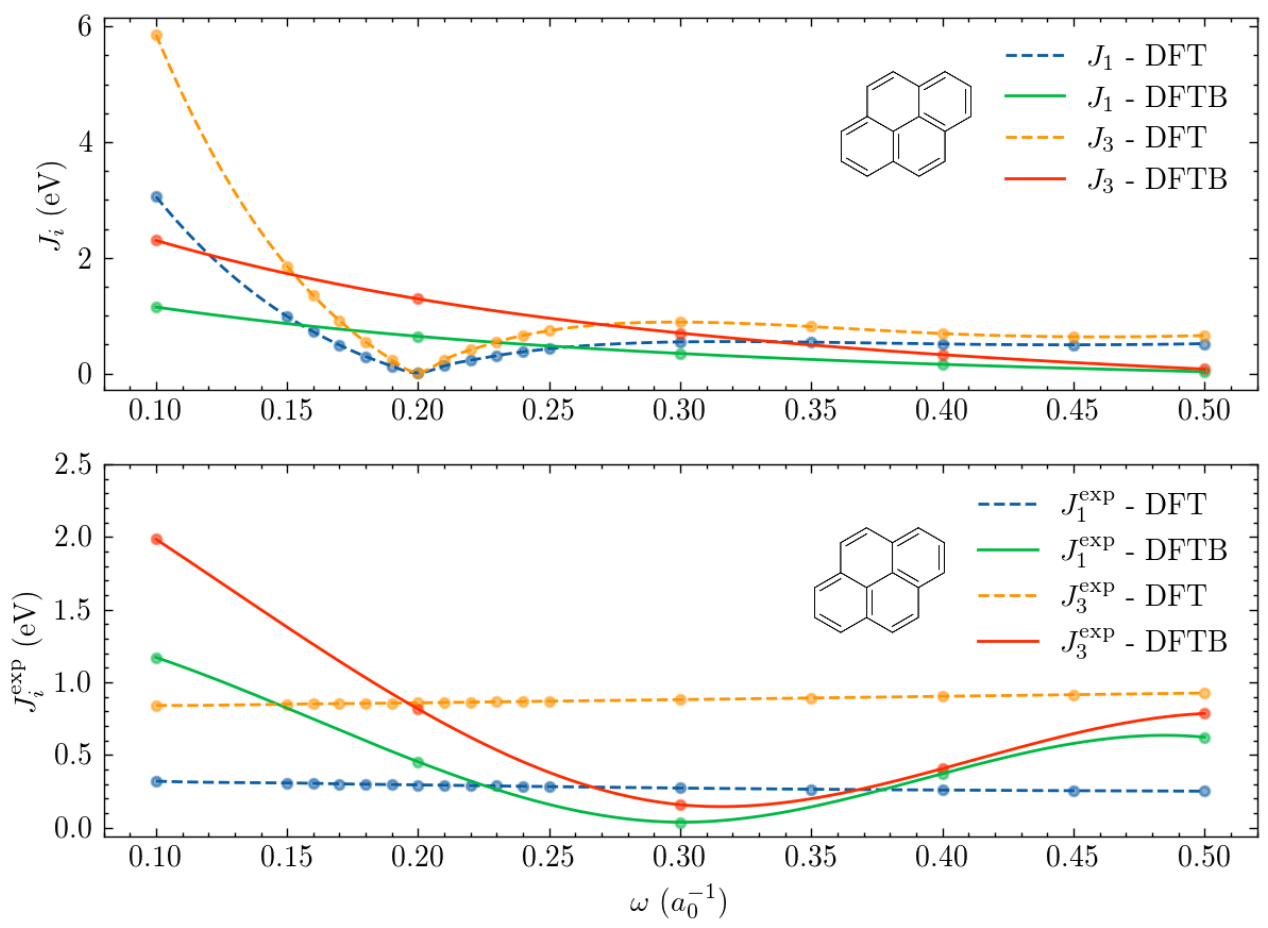

Figura 3.6: Critérios de otimização $J_{1}$ e $J_{3}$ em função do parâmetro de longo alcance $(\omega)$ para a molécula de pireno. No painel superior os valores foram obtidos utilizando o potencial de ionização $(I P)$ e a afinidade eletrônica $(E A)$ calculados, enquanto no painel inferior foram utilizados os valores experimentais. 
Em ambas as figuras, os gráficos superiores dizem respeito aos valores obtidos utilizando o potencial de ionização e a afinidade eletrônica calculados, enquanto os gráficos inferiores utilizam $I P$ e $E A$ experimentais em fase gasosa [90, 91, 92, 93]. As linhas pontilhadas correspondem aos valores obtidos via DFT enquanto as linhas sólidas dizem respeito a DFTB. Cada um dos pontos é um cálculo e as curvas são interpolações dos mesmos.

De imediato é possível verificar que os hidrocarbonetos ${ }^{5}$ se comportam de maneira semelhante. A calibração utilizando os valores experimentais reduz o $\omega$ ótimo obtido via $D F T B$, passando de $0.5 a_{0}^{-1}$ para $0.3 a_{0}^{-1}$, e acaba por diminuir o erro nos potenciais de ionização teóricos $\left(I P=E_{N-1}-E_{N}\right)$ dos três compostos, como é apresentado na Tabela 3.8.

\begin{tabular}{c|c|c|c} 
Sistema & $\omega\left(a_{0}^{-1}\right)$ & $\mathrm{IP}(\mathrm{eV})$ & $\operatorname{IP}_{\mathrm{EXP}}(\mathrm{eV})$ \\
\hline \multirow{2}{*}{ Naftaleno } & 0.3 & 8.618 & \multirow{2}{*}{$8.144(1)$} \\
& 0.5 & 8.889 & \\
\hline \multirow{2}{*}{ Antraceno } & 0.3 & 7.830 & $7.439(6)$ \\
& 0.5 & 8.094 & \\
\hline \multirow{2}{*}{ Pireno } & 0.3 & 7.812 & $7.426(1)$ \\
& 0.5 & 8.082 &
\end{tabular}

Tabela 3.8: Potenciais de ionização (IP) das moléculas de naftaleno, antraceno e pireno obtidos via $D F T B$. As linhas com $\omega=0.5 a_{0}^{-1}$ dizem respeito ao valor otimizado a partir das energias verticais enquanto $\omega=0.3 a_{0}^{-1}$ utiliza dos dados experimentais. Os valores entre parênteses na coluna $\mathrm{IP}_{\mathrm{EXP}}$ dizem respeito as incertezas experimentais.

As EAs, assim como os IPs, diminuem conforme $\omega$ diminui mas as variações são inferiores a $0.05 \mathrm{eV}$. Outro ponto positivo diz respeito a geometria otimizada via $D F T B$, cujas diferenças em comparação à otimização via $D F T$, apresentadas no Apêndice L, também diminuem quando $\omega$ passa a ser $0.3 a_{0}^{-1}$.

Ao adotar os potenciais experimentais nos cálculos em nível DFT os critérios $J_{i}$ se mantêm aproximadamente constantes, como é observado nos painéis inferiores das Figuras 3.5 e 3.6. Por mais que não haja motivo para que os pontos de mínimo sejam depressões acentuadas, o comportamento constante dos critérios em torno de um valor não nulo falha em fornecer um

\footnotetext{
${ }^{5}$ Os resultados para o antraceno não foram apresentados, mas os valores ótimos obtidos foram iguais aos dos outros dois compostos.
} 
valor otimizado de $\omega$ e indica que (independentemente do valor de $\omega$ ) o Teorema de Janak não é rigorosamente satisfeito. Desta forma, a utilização dos critérios $J_{i}$ com os valores experimentais de $I P$ e $E A$ na $D F T$ não é suficiente para os HPAs, necessitando recorrer à outros procedimentos de otimização.

Seguindo com a otimização de $\omega$, é importante discorrer sobre a correção de longo alcance e discutir o impacto do parâmetro em diferentes propriedades. Uma vez que a decomposição do potencial de Coulomb em contribuições de curto e longo alcance configura, por si só, uma correção no comportamento assintótico do funcional, já que no limite em que $\omega r_{12} \rightarrow \infty^{6}$ recuperamos o termo de Hartree exato, refinamentos como a redução do erro de auto-interação [83] e a descrição adequada de estados de transferência de carga [58] não devem, a priori, depender exclusivamente da escolha de $\omega$. Em comparação com conjuntos de dados [94], os erros de cálculos LC-DFT associados às energias de atomização, barreiras de reação, IPs e EAs, apresentam dependências distintas com relação ao $\omega[84,94]$. Como exemplo, os valores que minimizam cada grandeza para o funcional LC-PBE são, respectivamente, 0.37, 0.40, 0.32 e $0.08 a_{0}^{-1}$. Nesse contexto, a invariância dos critérios em relação ao $\omega$ observada para $D F T$, sugere que a otimização seja feita de acordo com alguma propriedade de interesse, e.g., a comparação do próprio espectro de absorção com dados experimentais ou outros cálculos mais sofisticados.

Confrontando os resultados entre os dois métodos verificamos que os cálculos via $D F T B$ apresentam valores otimizados de $\omega$ maiores com relação à DFT. Tal comportamento aponta que no formalismo semi-empírico a contribuição de longo alcance é mais expressiva, como é ilustrado na Figura 3.7. No painel (a) são apresentados os kernels das integrais de troca de curto e longo alcance do funcional $\omega$ B97X-D, introduzidos nas equações (2.24) e (2.25), para $\omega=0.1$ e 0.5. De maneira análoga, os kernels do funcional LC-BNL são exibidos no painel (b). No painel (c) são comparados os kernels das duas metodologias (para $\omega=0.5$ ) com o termo de Coulomb, enquanto no (d) são apresentadas as funções de atenuação das energias de correlação (ver equações (C.2) e (2.70)).

\footnotetext{
${ }^{6}$ Aqui $r_{12}=\left\|\boldsymbol{r}_{1}-\boldsymbol{r}_{2}\right\|$ segue a definição introduzida em (2.23).
} 

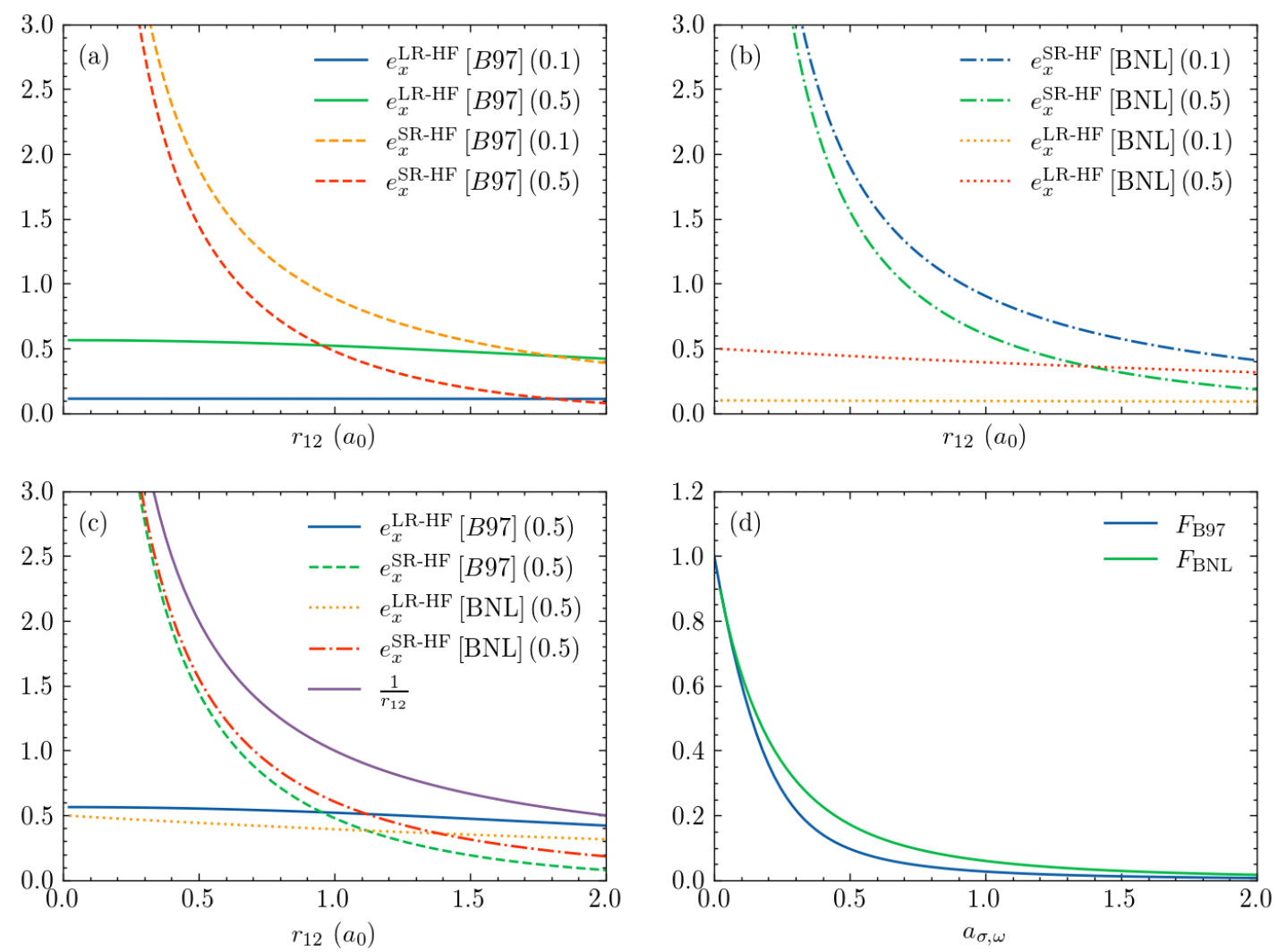

Figura 3.7: Comportamento das contribuições de curto e longo alcance do termo de Coulomb. Nas legendas o número entre parênteses corresponde ao valor de $\omega$. (a) Funções $\frac{\operatorname{erf}\left(\omega r_{12}\right)}{r_{12}}$ e $\frac{\operatorname{erfc}\left(\omega r_{12}\right)}{r_{12}} \operatorname{com} \omega=0.1$ e 0.5 . (b) Funções $\frac{e^{-\omega r_{12}}}{r_{12}}$ e $\frac{1-e^{-\omega r_{12}}}{r_{12}} \operatorname{com} \omega=0.1$ e 0.5 . (c) Comparação entre as decomposições para um mesmo valor de $\omega$. (d) Funções de atenuação das energias de troca $E_{x}^{\mathrm{SR}-\mathrm{B} 97}$ (C.2) e $E_{x}^{\omega, L D A}(2.70)$.

Para ambos os funcionais verificamos que o aumento de $\omega$ intensifica a contribuição de longo alcance, mas como as decomposições do potencial de Coulomb são distintas, para uma mesma densidade eletrônica com mesmo $\omega$ o LC-BNL fornece energias de longo alcance inferiores ao $\omega$ B97X-D, como é exemplificado no painel (c). Uma vez que as funções de atenuação se comportam de maneira semelhante, esperamos que as energias de troca do gás de elétrons livres que compõem ambos os modelos $\left(E_{x}^{\mathrm{SR}-\mathrm{B} 97}\right.$ (C.1) e $\left.E_{x}^{\omega, L D A}(2.69)\right)$ sejam praticamente iguais. Quando juntamos esses aspectos ao fato de que o LC-BNL implementado no código DFTB + não possui a contribuição de troca de curto alcance (ver $E_{x}^{\omega, H F}(2.71)$ ), é possível que os maiores valores ótimos de $\omega$ obtidos para $D F T B$, em comparação à DFT, busquem compensar essa contribuição, uma vez que a energia de troca do gás de elétrons livres por si só é deficiente na caracterização de moléculas pequenas, resultando em parâmetros de longo alcance maiores quando comparados ao $\omega$ B97X-D. 
Um último ponto diz respeito a proximidade entre os parâmetros ótimos dos três compostos dentro de um mesmo método, presente tanto via $D F T$ quanto via $D F T B$. Essa ocorrência sugere que moléculas quimicamente similares apresentam mesmo parâmetro de longo alcance ideal, e está de acordo com a utilização de um mesmo $\omega$ para moléculas pequenas, e.g., o uso de $\omega=0.3 a_{0}^{-1}$ por Kranz et al. [36] para a caracterização acurada de energias de excitação e ionização. Desta forma, acreditamos que é razoável utilizar dos parâmetros de longo alcance otimizados no presente trabalho para o estudo de outros HPAs.

\subsubsection{DTP-IC-4Ph}

Devido ao alto custo computacional dos cálculos envolvendo as moléculas aceitadoras, as otimizações foram realizadas somente via $D F T B$, e devido a ausência de $I P$ e $E A$ experimentais, ambos foram determinados apenas teoricamente. Tendo em vista a aplicação experimental, as cadeias laterais $\mathrm{C}_{6} \mathrm{H}_{13}$ não são cromóforos, e a adição das mesmas nos aceitadores visa apenas aumentar a solubilidade do composto [95]. A fim de investigar o impacto dessa adição no que diz respeito à otimização do parâmetro $\omega$, a Tabela 3.9 traz os critérios de otimização para o DTP-IC-4Ph com e sem as cadeias laterais.

\begin{tabular}{c|cc|cc} 
& \multicolumn{2}{|c|}{ DTP-IC-4Ph } & \multicolumn{2}{c}{ DTP-IC-4Ph ${ }^{\dagger}$} \\
\hline$\omega\left(a_{0}^{-1}\right)$ & $J_{1}(\mathrm{eV})$ & $J_{3}(\mathrm{eV})$ & $J_{1}(\mathrm{eV})$ & $J_{3}(\mathrm{eV})$ \\
\hline 0.1 & 0.128 & 0.254 & 0.066 & 0.134 \\
0.2 & 0.469 & 0.843 & 0.271 & 0.453 \\
0.3 & 0.664 & 1.168 & 0.465 & 0.804 \\
0.4 & 0.787 & 1.365 & 0.587 & - \\
0.5 & 0.862 & 1.486 & 0.665 & 1.065 \\
\hline
\end{tabular}

Tabela 3.9: Critérios de otimização $J_{1}$ e $J_{3}$ para o composto DTP-IC-4Ph variando o parâmetro de longo alcance $(\omega)$ no intervalo de 0.1 até $0.5 a_{0}^{-1}$. A coluna DTP-IC- $4 \mathrm{Ph}^{\dagger}$ diz respeito aos valores obtidos na ausência de cadeias laterais.

Em ambos os casos, dentre os valores disponíveis, o valor ideal é de $\omega=$ $0.1 a_{0}^{-1}$, porém as diferenças entre os critérios para cada composto sugerem que o mínimo do DTP-IC-4Ph ${ }^{\dagger}$ esteja mais próximo de $0.1 a_{0}^{-1}$ do que o do DTP-IC-4Ph, o que a princípio indica uma, pequena mas existente, alteração no parâmetro ótimo. De maneira análoga às curvas apresentadas 
para os hidrocarbonetos nas Figuras 3.5 e 3.6, a Figura 3.8 traz a interpolação dos valores de $J_{1}$ e $J_{3}$ em função de $\omega$, ilustrando que a substituição dos radicais hexil $\left(\mathrm{C}_{6} \mathrm{H}_{13}\right)$ por átomos de hidrogênio mantém os critérios $J_{i}$ monotonicamente crescentes.

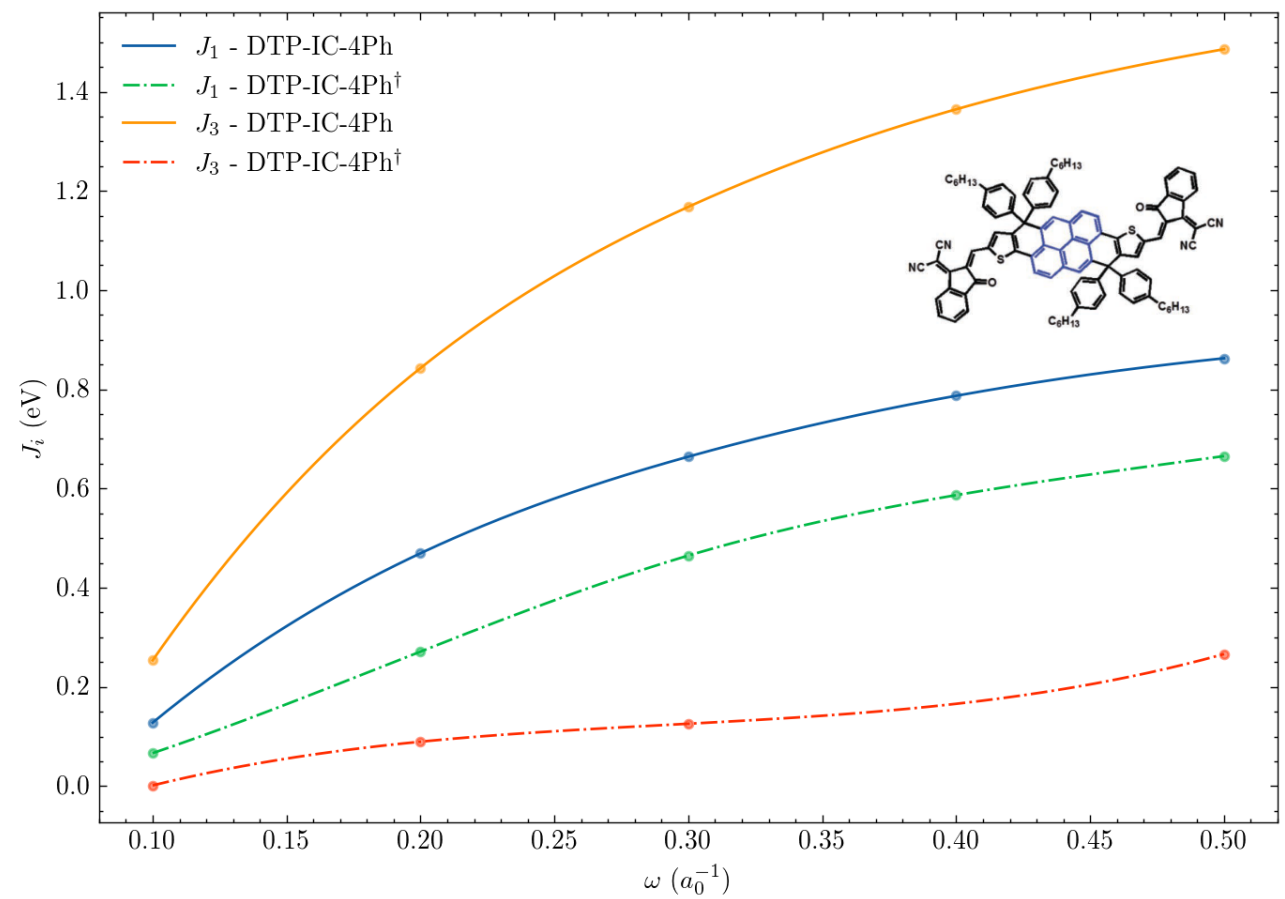

Figura 3.8: Critérios de otimização $J_{1}$ e $J_{3}$ em função do parâmetro de longo alcance $(\omega)$ para o composto DTP-IC-4Ph com e sem cadeias laterais. O símbolo ${ }^{\dagger}$ identifica o composto sem cadeias laterais.

As curvas tracejadas ilustram que a ausência de cadeias laterais reduz os critérios não só para $\omega=0.1 a_{0}^{-1}$ mas ao longo de todo o intervalo. Ao unir os valores inferiores dos $J_{i}$ do composto sem cadeias laterais, com a ausência de atividade ótica dessas cadeias e o ganho computacional obtido pela redução de 72 átomos, somos motivados a utilizar da versão reduzida do aceitador nos demais cálculos.

Antes de seguir com a investigação sobre o impacto das cadeias laterais, é importante destacar as diferenças entre as otimizações das duas classes de compostos. O contraste entre os valores otimizados de $\omega=0.1 a_{0}^{-1}$ para o aceitador e $\omega=0.5 a_{0}^{-1}$ para os HPAs sugere que o número de átomos, assim como a presença de outros ametais ( $\mathrm{O}, \mathrm{N}$ e $\mathrm{S})$, impactam significativamente na escolha de $\omega$. As curvas estritamente decrescentes dos hidrocarbonetos (Figuras 3.5 e 3.6) em oposição às crescentes para o DTP-IC-4Ph (Figura 3.8) sugerem que a contribuição de troca exata é mais 
relevante para moléculas pequenas, o que pode ser razoável uma vez que a energia LDA tende a fornecer melhores resultados para sistemas grandes, em especial sólidos [31].

Seguindo com a investigação do impacto das cadeias laterais, ao comparar as energias dos orbitais HOMO e LUMO os resultados são semelhantes. Como podemos observar na Tabela 3.10, as variações na energia do HOMO tendem a aumentar conforme $\omega$ aumenta porém se mantendo inferiores a $0.1 \mathrm{eV}$. Para um mesmo $\omega$, as energias dos LUMOs dos dois compostos são aproximadamente iguais, o que estabelece boa concordância entre os gaps de energia e reproduz o padrão de crescimento dos mesmos conforme aumentamos $\omega$.

\begin{tabular}{c|ccc|ccc} 
& \multicolumn{3}{|c|}{ DTP-IC-4Ph } & \multicolumn{3}{c}{ DTP-IC-4Ph $^{\dagger}$} \\
\hline$\omega\left(a_{0}^{-1}\right)$ & $\epsilon_{\text {HOMO }}(\mathrm{eV})$ & $\epsilon_{L U M O}(\mathrm{eV})$ & $E_{\text {gap }}^{H-L}(\mathrm{eV})$ & $\epsilon_{H O M O}(\mathrm{eV})$ & $\epsilon_{L U M O}(\mathrm{eV})$ & $E_{\text {gap }}^{H-L}(\mathrm{eV})$ \\
\hline 0.1 & -5.768 & -2.606 & 3.162 & -5.805 & -2.603 & 3.202 \\
0.2 & -6.358 & -2.184 & 4.174 & -6.410 & -2.183 & 4.227 \\
0.3 & -6.764 & -1.936 & 4.828 & -6.826 & -1.938 & 4.888 \\
0.4 & -7.042 & -1.783 & 5.259 & -7.110 & -1.787 & 5.323 \\
0.5 & -7.242 & -1.666 & 5.576 & -7.317 & -1.672 & 5.645 \\
\hline
\end{tabular}

Tabela 3.10: Energias dos orbitais HOMO $\left(\epsilon_{H O M O}\right)$ e LUMO $\left(\epsilon_{L U M O}\right)$ assim como suas diferenças $E_{\text {gap }}^{H-L}$ calculadas para o composto DTP-IC-4Ph variando o parâmetro de longo alcance $(\omega)$ no intervalo de 0.1 até $0.5 a_{0}^{-1}$. A coluna DTP-IC- $4 \mathrm{Ph}^{\dagger}$ diz respeito aos valores obtidos na ausência de cadeias laterais.

Ao confrontar os valores para o parâmetro de longo alcance otimizado com dados experimentais obtidos utilizando técnicas de voltametria cíclica em filme fino [15], as diferenças ficam evidentes. Enquanto as energias do HOMO são próximas, $\epsilon_{H O M O}^{\exp }=-5.63 \mathrm{eV}$ para a experimental e $\epsilon_{H O M O}=$ -5.77 eV para a teórica, a energia do LUMO que se mostra insensível frente à remoção das cadeias laterais, é a principal responsável pela diferença entre os resultados, uma vez que o valor experimental é de $\epsilon_{L U M O}^{\exp }=-3.85$ $\mathrm{eV}$ em desarmonia com $\epsilon_{L U M O}=-2.61 \mathrm{eV}$ apresentado na tabela. Como consequência o gap experimental é de $E_{\text {gap }}^{\exp }=1.78 \mathrm{eV}$ em oposição ao calculado $E_{\text {gap }}=3.16 \mathrm{eV}$.

Tais diferenças podem ser entendidas a partir de diferentes argumentos. No que diz respeito ao formalismo da DFT, ainda que diante da densidade exata os orbitais HOMO e LUMO de Kohn-Sham reproduzam IP e EA 
com ótima precisão [96], tais densidades não são acessíveis. A utilização de aproximações no termo de correlação e troca compromete o desempenho de funcionais GGA, meta-GGA e até mesmo híbridos, e.g., ocasionando erros da ordem de $3 \mathrm{eV}$ na energia do HOMO para moléculas pequenas utilizando o funcional híbrido B3LYP [97]. Apenas após a incorporação das correções de longo alcance as energias dos orbitais passam a ser qualitativamente corretas [58]. A redução do erro de auto-interação no cálculo do kernel $f_{x c}$ e minimiza o problema da dependência da energia dos orbitais com relação ao número de ocupação [98], resultando em energias, em nível DFT, comparáveis à métodos ab initio para uma gama de compostos pequenos [58]. O benefício na DFTB fica claro quando comparamos as energias do HOMO na presença e ausência da correção. A Tabela 1 no material suplementar do trabalho de Lutsker et al. [35] utiliza do conjunto de parâmetros mio-1-1 [99] e compara $\epsilon_{H O M O}$ de diversos sistemas $\pi$-conjugados. Em destaque, os 3 HPAs naftaleno, antraceno e pireno, cujos valores $5.98,5.52$ e $5.59 \mathrm{eV}$ passam a ser, respectivamente, 8.22, 7.55 e 7.56 $\mathrm{eV}$, evidenciam o ganho quando confrontamos com os valores experimentais $8.14,7.44$ e $7.43 \mathrm{eV}$. Como foi apresentado, essa melhoria também é observada para o aceitador porém a energia do LUMO permanece distante.

Ainda na comparação com os dados experimentias, em relação aos efeitos do ambiente, por mais que a presença de solvente não altere substancialmente a energia dos orbitais HOMO e LUMO dos HPAs, como será discutido na Seção 3.5, por se tratar de moléculas maiores com outros átomos e grupos funcionais, que não sejam apenas carbonos e hidrogênios com suas estruturas ressonantes, é de se esperar que haja modificações no caráter e nas energias dos orbitais decorrentes da presença de outras unidades do aceitador. Entretanto, para as discrepâncias substanciais na energia do LUMO também devemos levar outros pontos em consideração. Além do tamanho do aceitador, uma possível causa pode ser atribuída a base mínima de funções de Slater, que não leva em conta funções difusas que são importantes para a determinação de afinidades eletrônica $(E A)$ [100], e portanto, no espírito do Teorema de Janak, da energia do LUMO. Como foi discutido anteriormente, a utilização da base mínima de funções gaussianas promove erros substanciais quando comparada com as demais bases atômicas, e, por mais que aqui sejam funções de Slater, o número pequeno de vetores da base compromete o desempenho do ponto de vista variacional. 


\subsubsection{PBDB-T}

Para o polímero doador PBDB-T foi realizado o mesmo procedimento de otimização do aceitador mas considerando apenas um monômero. A partir da metodologia DFTB com a versão adaptada dos parâmetros de Slater-Koster (OB2+S), a geometria do composto neutro foi otimizada, e nessa geometria o cálculo das energias dos íons possibilita a determinação dos critério $J_{1}$ e $J_{3}$.

\begin{tabular}{c|cc|cc} 
& \multicolumn{2}{|c|}{ PBDB-T } & \multicolumn{2}{c}{ PBDB-T $^{\dagger}$} \\
\hline$\omega\left(a_{0}^{-1}\right)$ & $J_{1}(\mathrm{eV})$ & $J_{3}(\mathrm{eV})$ & $J_{1}(\mathrm{eV})$ & $J_{3}(\mathrm{eV})$ \\
\hline 0.1 & 0.033 & 0.081 & 0.397 & 0.815 \\
0.2 & 0.311 & 0.632 & 0.055 & 0.096 \\
0.3 & 0.528 & 1.057 & 0.158 & 0.331 \\
0.4 & 0.670 & 1.328 & 0.302 & 0.608 \\
0.5 & 0.762 & 1.502 & 0.398 & 0.789 \\
\hline
\end{tabular}

Tabela 3.11: Critérios de otimização $J_{1}$ e $J_{3}$ para o composto PBDB-T variando o parâmetro de longo alcance $(\omega)$ no intervalo de 0.1 até $0.5 a_{0}^{-1}$. A coluna PBDB-T ${ }^{\dagger}$ diz respeito aos valores obtidos na ausência de cadeias laterais.

Diferentemente dos compostos anteriores, de acordo com a Tabela 3.11 o parâmetro ótimo muda substancialmente quando desconsideramos as cadeias laterais $^{7}$, saindo de $\omega=0.1 a_{0}^{-1}$ para $\omega=0.2 a_{0}^{-1}$. No que diz respeito às energias dos orbitais, o doador se comporta de maneira análoga ao aceitador. À medida que aumentamos o parâmetro de longo alcance os orbitais HOMO passam a se tornar mais ligados em oposição aos orbitais LUMO. A Tabela 3.12 exemplifica tal comportamento e destaca a dependência diretamente proporcional entre o $E_{\text {gap }}^{H-L}$ e $\omega$.

\footnotetext{
${ }^{7}$ Neste caso o ganho computacional é ainda maior, reduzindo o número de átomos de 156 para 62.
} 


\begin{tabular}{c|ccc|ccc} 
& \multicolumn{3}{|c|}{ PBDB-T } & \multicolumn{3}{c}{ PBDB-T $^{\dagger}$} \\
\hline$\omega\left(a_{0}^{-1}\right)$ & $\epsilon_{\text {HOMO }}(\mathrm{eV})$ & $\epsilon_{L U M O}(\mathrm{eV})$ & $E_{\text {gap }}^{H-L}(\mathrm{eV})$ & $\epsilon_{\text {HOMO }}(\mathrm{eV})$ & $\epsilon_{L U M O}(\mathrm{eV})$ & $E_{\text {gap }}^{H-L}(\mathrm{eV})$ \\
\hline 0.1 & -5.460 & -1.471 & 3.989 & -5.621 & -1.623 & 3.998 \\
0.2 & -6.162 & -0.986 & 5.176 & -6.327 & -1.123 & 5.204 \\
0.3 & -6.644 & -0.719 & 5.926 & -6.816 & -0.855 & 5.961 \\
0.4 & -6.985 & -0.534 & 6.452 & -7.159 & -0.657 & 6.502 \\
0.5 & -7.227 & -0.431 & 6.796 & -7.404 & -0.552 & 6.852 \\
\hline
\end{tabular}

Tabela 3.12: Energias dos orbitais HOMO $\left(\epsilon_{H O M O}\right)$ e LUMO $\left(\epsilon_{L U M O}\right)$ assim como suas diferenças $E_{\text {gap }}^{H-L}$ calculadas para o composto PBDB-T variando o parâmetro de longo alcance $(\omega)$ no intervalo de 0.1 até $0.5 a_{0}^{-1}$. A coluna $\mathrm{PBDB}^{-\mathrm{T}^{\dagger}}$ diz respeito aos valores obtidos na ausência de cadeias laterais.

De acordo com Zhao et al. [25], um filme fino do polímero apresenta energias $-5.23 \mathrm{eV}$ e $-3.18 \mathrm{eV}$ para os orbitais HOMO e LUMO, respectivamente. A diferença entre o gap experimental e o teórico é de $1.94 \mathrm{eV}$ e novamente pode ser atribuída ao valor teórico superestimado da energia do LUMO. Ainda que os cálculos sofram das mesmas limitações do formalismo, discutidas na subseção anterior, por se tratar de uma comparação entre monômero e polímero outros efeitos são relevantes. O aumento do número de oligômeros na descrição de um polímero impacta no parâmetro de longo alcance [40] e produz o efeito conhecido de diminuição do gap do material. No trabalho de Larsen [41], as variações no gap devido ao acréscimo de oligômeros chegam em até $5 \mathrm{eV}$, em especial, o polímero P7 cuja estrutura central apresenta grande semelhança com a do PBDB-T, apresenta redução de cerca de $1 \mathrm{eV}$ entre o gap do monômero e o de 10 oligômeros. Desta forma, fica evidente que boa parte do gargalo se encontra no tamanho do composto, minimizando a discrepância entre os resultados obtidos no presente trabalho e os dados experimentais.

\subsection{Comparação entre metodologias}

Nos últimos anos foram reportados trabalhos que comparam o formalismo SCC-DFTB para diferentes classes de sistemas. No estudo de nanoesferas de $\mathrm{TiO}_{2}$, realizado por Selli et al. [101], a metodologia apresenta alta compatibilidade com DFT em nível B3LYP, tanto do ponto de vista estrutural quanto eletrônico, reproduzindo os valores experimentais das bandas de energia e até mesmo o efeito de estabilidade decorrente do tratamento 
térmico. A caracterização de estados excitados se mostrou acurada, com destaque para hidrocarbonetos aromáticos e heterocíclicos, performando melhor que outros métodos semi-empíricos [102].

Diante da implementação da correção de longo alcance, a metodologia se beneficia das mesmas melhorias que a DFT, reduzindo erros de autointeração e aperfeiçoando a caracterização de estados delocalizados [35]. O baixo custo computacional é mantido, permitindo a realização de dinâmicas moleculares durante maiores intervalos de tempo e com o mínimo de perda na precisão, e.g., a caracterização do espectro de éxcitons dos compostos orgânicos coletores de luz estudados por Bold et al. [37]. Contudo, limitações metodológicas como a expansão do funcional de energia até o termo de segunda ordem em torno da energia de átomos livres (ver Subseção 2.4.1), e limitações práticas como a ausência de funções de polarização, decorrente da utilização de base mínima (ver Subseção 2.4.2), continuam presentes e motivam a validação da metodologia ao menos para os compostos de interesse. Os hidrocarbonetos naftaleno, antraceno e pireno foram estudados, buscando validar os resultados DFTB frente à DFT e dando continuidade na análise iniciada na Seção 3.2.

Uma das propriedades eletrônicas relevantes que é afetada pela mudança do parâmetro de longo alcance é a energia do gap, que reflete as variações nas energias dos orbitais de fronteira. A Tabela 3.13 confronta os gaps HOMO-LUMO teóricos com os valores experimentais.

\begin{tabular}{c|cc|cc|c} 
& \multicolumn{2}{|c|}{$\boldsymbol{D F T}$} & \multicolumn{2}{|c|}{$\boldsymbol{D F T B}$} & Exp \\
\hline Sistema & $\omega\left(a_{0}^{-1}\right)$ & $E_{\text {gap }}^{H-L}(\mathrm{eV})$ & $\omega\left(a_{0}^{-1}\right)$ & $E_{\text {gap }}^{H-L}(\mathrm{eV})$ & $E_{\text {gap }}^{H-L}(\mathrm{eV})$ \\
\hline \multirow{2}{*}{ Naftaleno } & 0.2 & 8.578 & 0.5 & 9.324 & \multirow{2}{*}{$0.344(50)$} \\
& & & 0.3 & 8.298 & \\
\hline \multirow{2}{*}{ Antraceno } & 0.2 & 7.136 & 0.5 & 7.759 & \multirow{2}{*}{$0.909(21)$} \\
& & & 0.3 & 6.868 & \\
\hline \multirow{2}{*}{ Pireno } & 0.2 & 7.289 & 0.5 & 7.805 & \multirow{2}{*}{$0.020(10)$} \\
& & & 0.3 & 6.937 & \\
\hline
\end{tabular}

Tabela 3.13: Gap de energia obtido experimentalmente e através dos métodos DFT e $D F T B$ adotando o parâmetro de longo alcance otimizado. Na coluna DFTB são apresentados os valores otimizados teórico e experimental, respectivamente. Os valores entre parênteses na coluna Exp dizem respeito as incertezas experimentais. 
Na primeira coluna são apresentados os resultados via $D F T$, na segunda via $D F T B$ e por fim os experimentais. Dentro da coluna $D F T B$, na primeira linha o valor de $\omega$ é otimizado com base no $I P$ e $E A$ teóricos (diferença vertical entre a energia dos íons e o neutro), acompanhado da energia do gap correspondente, enquanto na segunda linha o valor é otimizado a partir de $I P$ e $E A$ experimentais. Com exceção dos casos em que $\omega=0.5 a_{0}^{-1}$, a compatibilidade entre os gaps de energia $D F T, D F T B$ e experimental é favorável, com destaque para os gaps DFTB com $\omega=0.3 a_{0}^{-1}$, que para os compostos menores se encontram dentro do intervalo de confiança da incerteza experimental. Por mais que a otimização via dados experimentais seja construída de modo a minimizar a diferença com relação ao gap experimental, uma vez que esses gaps experimentais são determinados a partir de $I P$ e $E A$, o mesmo comportamento não é observado no caso da DFT que se mantém aproximadamente invariante, como foi discorrido previamente (ver Subseção 3.2.1).

Acerca do espectro de estados excitados, é observado o blue shift do DFTB em relação ao DFT, um deslocamento rígido para maiores energias. Na Figura 3.9 são apresentados espectros de absorção da molécula de naftaleno, todos na geometria otimizada via DFT.

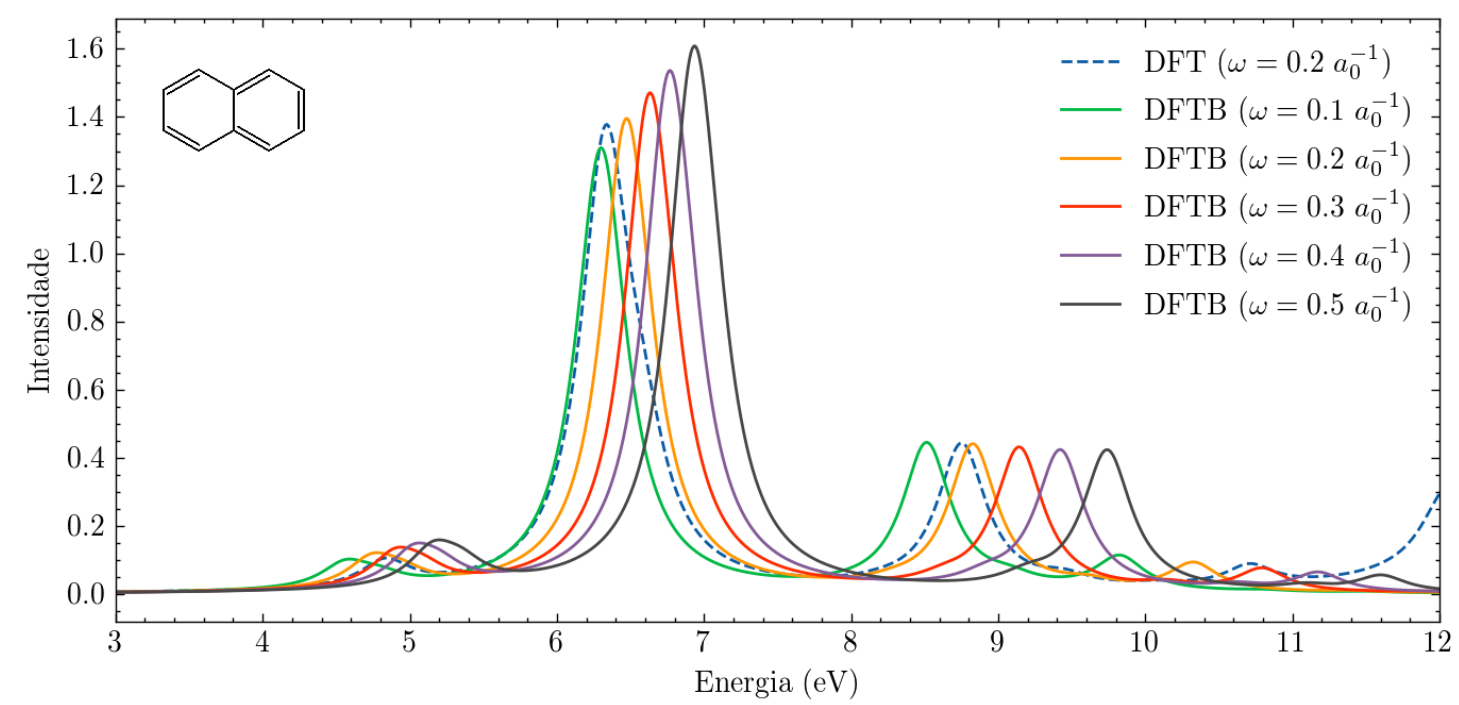

Figura 3.9: Espectros de absorção da molécula de naftaleno obtidos utilizando ambas os métodos. Os cálculos de estados excitados foram realizados na geometria otimizada via DFT/ $\omega$ B97X-D/6-31G(d,p). Foram considerados 100 estados excitados em cada cálculo.

Além do deslocamento horizontal, há uma tendência de aumento das forças de oscilador dos primeiros picos com o aumento de $\omega$, evidente para energias 
em torno de 5 e de $6.5 \mathrm{eV}$. Outra característica consiste no adensamento de estados não acessíveis via absorção de radiação, que acaba por incorporar transições com força de oscilador nula (ver Apêndice M). Na figura acima tal comportamento pode ser verificado, uma vez que foi considerado o mesmo número de estados excitados em todos os cálculos, mas apenas para a curva tracejada é possível observar o início de um novo pico em torno de $12 \mathrm{eV}$. Em relação ao levantamento do espectro de absorção, a presença desses estados não acessíveis oticamente não compromete os resultados pois apenas demanda um número maior de estados excitados para os cálculos $D F T B$ em relação ao $D F T$.

O impacto de $\omega$ na geometria do estado fundamental é discutido no Apêndice L mas de maneira geral é pequeno, e quando associado ao melhoramento no espectro decorrente do uso de $\omega=0.3 a_{0}^{-1}$ em oposição à $\omega=0.5 a_{0}^{-1}$ reforça a ideia original de otimização do parâmetro de longo alcance utilizando $I P$ e $E A$ experimentais como meio de melhorar a qualidade dos resultados. Os ganhos na otimização de geometria dos compostos, no gap de energia HOMO-LUMO e até mesmo nos cálculos verticais de $I P$ e $E A$, apresentados na Seção 3.2.1, se tornam ainda mais relevantes uma vez que os espectros de absorção seguem a mesma tendência, sugerindo um ganho global.

\subsection{Espectros de absorção}

Em vista da utilização dos compostos estudados no contexto de dispositivos fotovoltaicos, o levantamento dos espectros de absorção é de grande importância e configura um dos objetivos centrais do presente projeto, tanto na validação da metodologia empregada quanto na comparação com dados experimentais. Assim como foi realizado nas seções anteriores, os espectros são determinados a partir dos cálculos dependentes do tempo. Em ambos os formalismos, para cada estado excitado do composto é associada uma energia de transição assim com a respectiva força de oscilador. A geometria e os gradientes são otimizados com relação ao estado eletrônico de interesse, ${ }^{8}$ uma lorentziana com largura de $0.2 \mathrm{eV}^{9}$ é centrada em cada

\footnotetext{
${ }^{8}$ As otimizações foram realizadas em torno do estado fundamental, acarretando em resultados precisos para bandas de menor energia.

${ }^{9} \mathrm{~A}$ largura das lorentzianas tende a ser empírica e tipicamente busca reproduzir o espectro experimental, descrevendo o erro associado à medição em conjunto ao tempo de vida frente ao decaimento radiativo espontâneo. A escolha em $0.2 \mathrm{eV}$ suaviza os espectros que não levam em conta dados experimentais e se mostra adequada na reprodução do alargamento do espectro experimental da Figura 3.15.
} 
energia correspondente a um pico de excitação e a convolução sobre todo o intervalo de energia resulta no espectro de absorção (ver Apêndice N).

A abordagem teórica permanece a mesma, para os compostos pequenos os espectros foram calculados utilizando ambos os formalismos, enquanto os compostos doador e aceitador se restringem à DFTB. O ajuste e a convolução das lorentzianas foi realizado por meio do software Lorentz, gentilmente disponibilizado pela Profa. Dra. Kaline Coutinho.

\subsubsection{Hidrocarbonetos poli-aromáticos}

Para os hidrocarbonetos, os espectros de absorção são apresentados nas Figuras 3.10, 3.11 e 3.12. As curvas tracejadas correspondem aos espectros obtidos via $D F T$ e as linhas sólidas via $D F T B$, onde tanto a otimização de geometria quanto o cálculo de estados excitados foram realizados com o mesmo método. Na legenda das figuras, ao lado do método utilizado é indicado entre parênteses o parâmetro de longo alcance adotado, que foi otimizado pelo procedimento descrito na Seção 3.2. Os espectros DFTB EXP $_{\text {. }}$ foram obtidos com os valores de $\omega$ otimizados a partir de dados experimentais de potencial de ionização e afinidade eletrônica, em oposição aos cálculos teóricos das energias dos íons $\left(E_{N \pm 1}\right)$ utilizados na otimização das demais curvas.

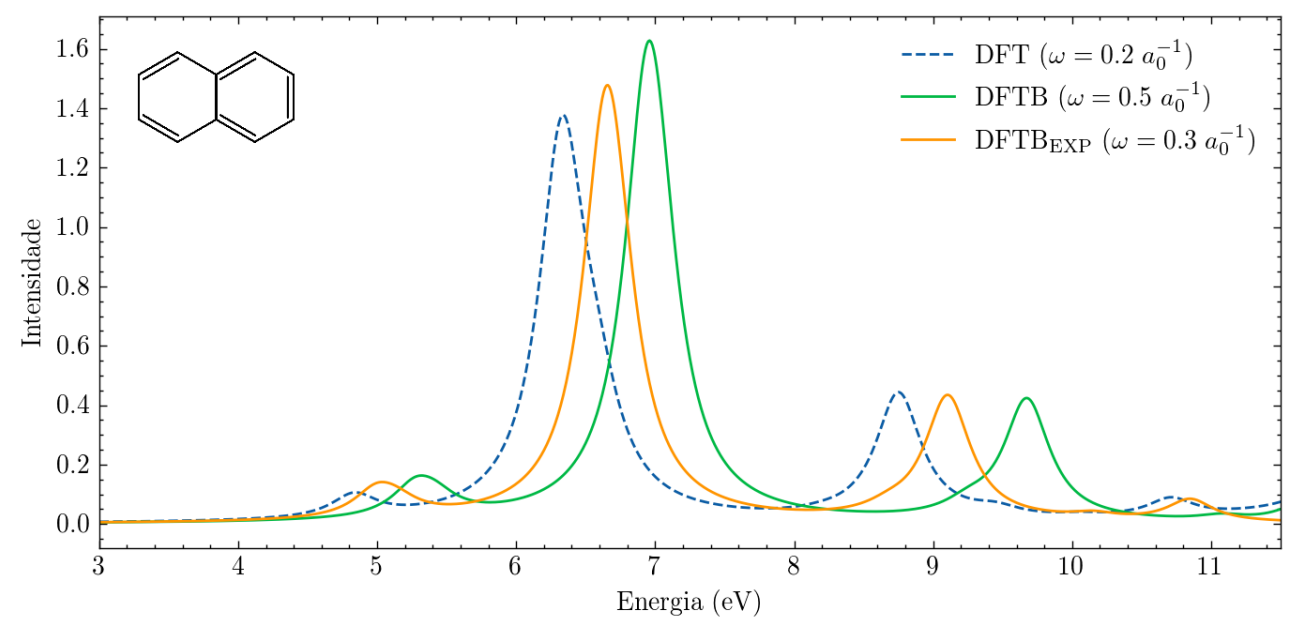

Figura 3.10: Espectros de absorção da molécula de naftaleno obtidos utilizando ambas as metodologias. A legenda faz referência aos métodos DFT e DFTB, utilizados tanto na otimização de geometria quanto no cálculo de estados. Foram considerados 100 estados excitados em cada cálculo. 


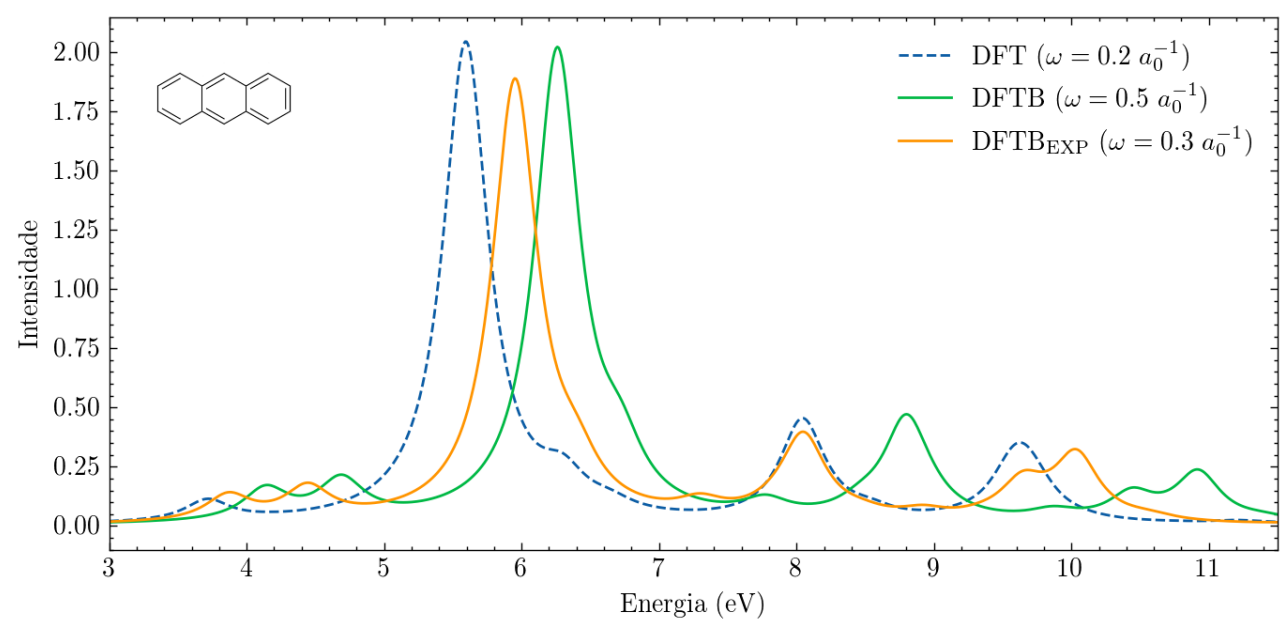

Figura 3.11: Espectros de absorção da molécula de antraceno obtidos utilizando ambas as metodologias. A legenda faz referência aos métodos DFT e DFTB, utilizados tanto na otimização de geometria quanto no cálculo de estados. Foram considerados 100 estados excitados em cada cálculo.

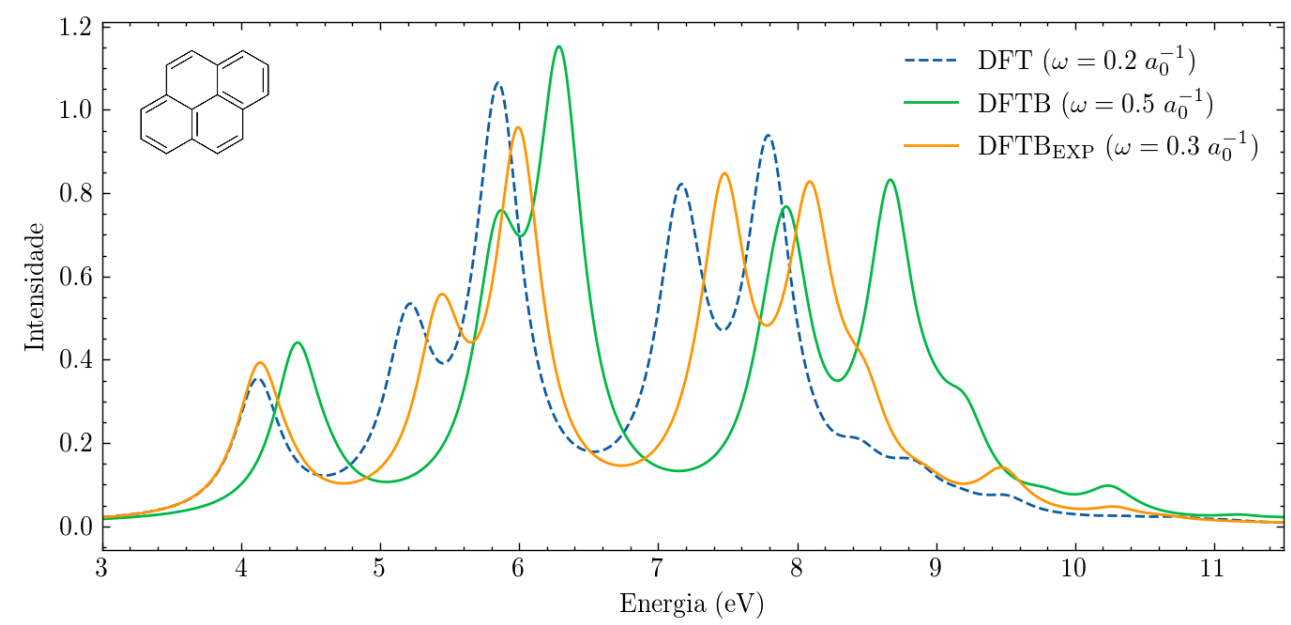

Figura 3.12: Espectros de absorção da molécula de pireno obtidos utilizando ambas as metodologias. A legenda faz referência aos métodos DFT e DFTB, utilizados tanto na otimização de geometria quanto no cálculo de estados. Foram considerados 100 estados excitados em cada cálculo.

No caso do naftaleno, as pequenas variações nas geometrias otimizadas com cada método resultam em curvas muito próximas às retratadas na seção anterior, de sorte que as diferenças observadas são essencialmente as mesmas da Figura 3.9, apresentando um blue shift do DFTB em relação ao DFT, com aumento na intensidade dos picos de menor energia. No que se refere ao deslocamento rígido, o mesmo comportamento é observado para os demais hidrocarbonetos, com pequena ressalva para os picos do antraceno em $8 \mathrm{eV}$ e do pireno em $4 \mathrm{eV}$, que sobrepõem as curvas DFT

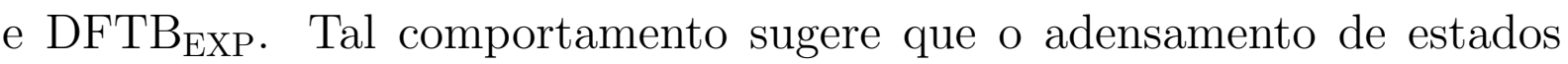


com força de oscilador nula (discutido na Seção 3.2) seja não uniforme, resultando em regiões do espectro com diferentes densidades de estados. Essas diferenças se manifestam ao considerar, por exemplo, o pico de baixa intensidade em torno de $6.5 \mathrm{eV}$ da molécula de antraceno no cálculo $D F T$, que é suavizado nas curvas $D F T B$ e $D F T B_{\text {EXP. Na mesma figura dos }}$ espectros do antraceno, outra manifestação das alterações na densidade de estados é observada no pico de maior energia, que se separa em dois quando passamos da abordagem DFT para a DFTB.

\subsubsection{DTP-IC-4Ph}

A fim de estudar o impacto da omissão das cadeias laterais no composto doador, foram levantados 100 estados excitados em ambas as composições apenas via DFTB, resultando nos espectros apresentados na Figura 3.13.

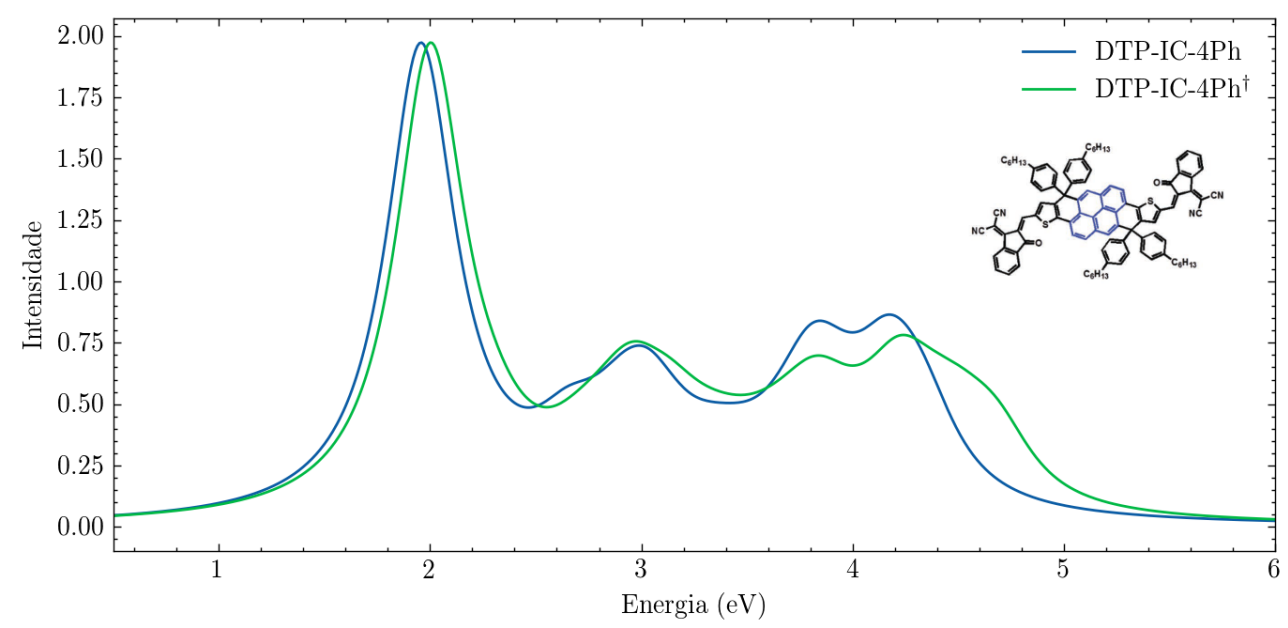

Figura 3.13: Espectros de absorção do composto DTP-IC-4Ph. A curva azul descreve o composto como apresentado na figura, enquanto a curva verde troca as cadeias laterais $\mathrm{C}_{6} \mathrm{H}_{13}$ por átomos de hidrogênio.

A curva azul diz respeito ao espectro da molécula com cadeias laterais enquanto a verde substitui os ramos $\mathrm{C}_{6} \mathrm{H}_{13}$ por átomos de hidrogênio. Em boa aproximação os espectros são compatíveis, com destaque para o primeiro pico, legitimando a adição de cadeias laterais como meio de aumentar a solubilidade da molécula sem afetar as propriedades óticas. Dada a aplicação do composto DTP-IC-4Ph em células solares, a concordância observada na região do infravermelho e do visível (ao redor do primeiro pico de absorção) alinhada à compatibilidade entre as energias dos orbitais HOMO e LUMO (ver Tabela 3.10) e o caráter dos mesmos, como é apresentado na Figura 3.14, valida o uso do modelo sem cadeias laterais. 


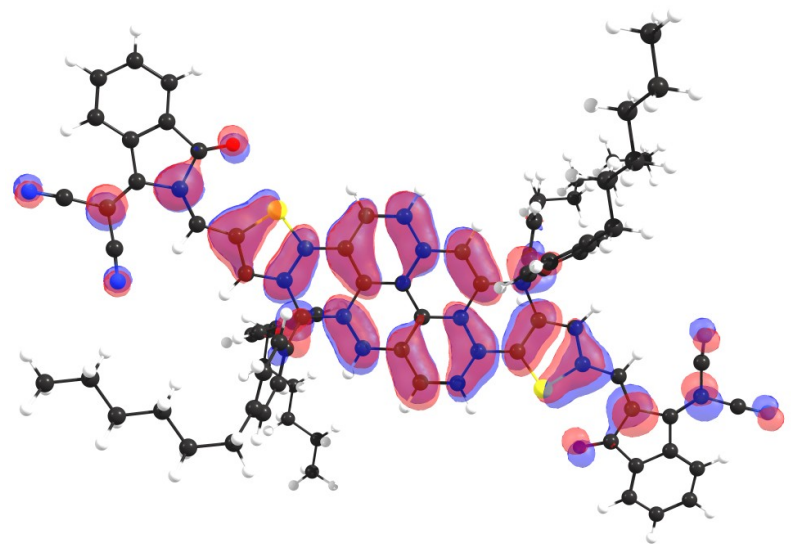

(a) (H) DTP-IC-4Ph

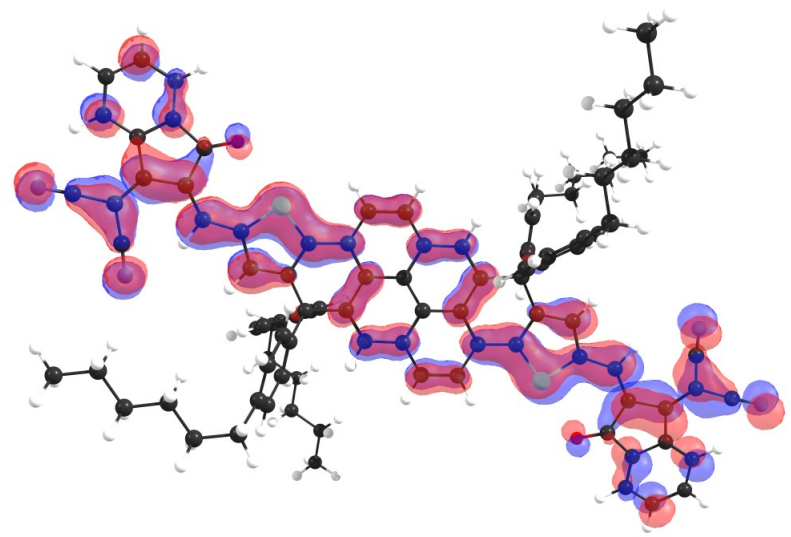

(c) (L) DTP-IC-4Ph

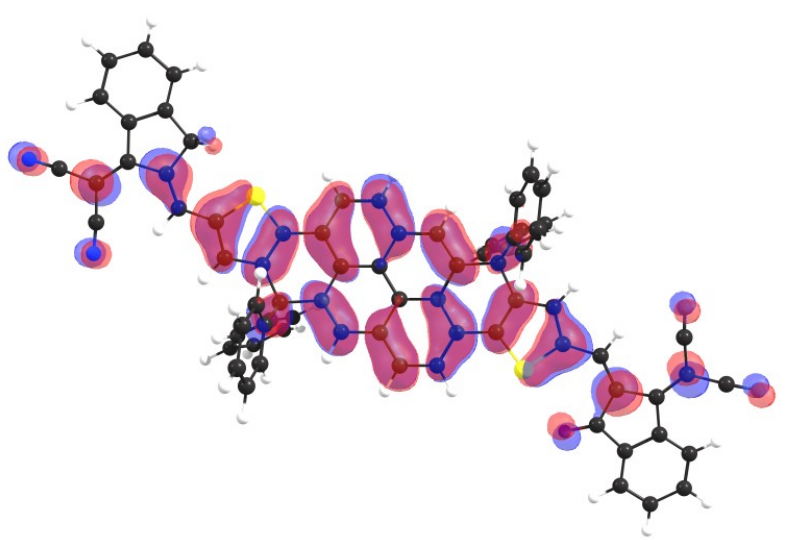

(b) (H) DTP-IC-4Ph ${ }^{\dagger}$

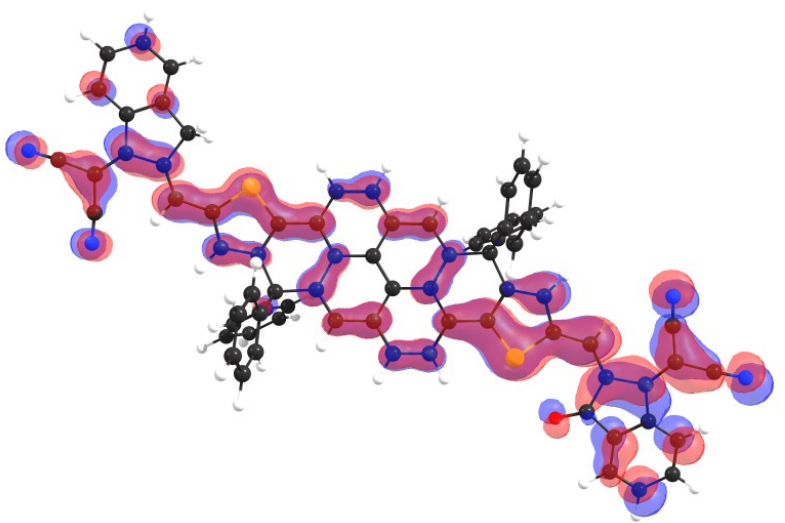

(d) (L) DTP-IC-4Ph ${ }^{\dagger}$

Figura 3.14: Isosuperfícies dos orbitais HOMO (painel superior) e LUMO (painel inferior) do aceitador DTP-IC-4Ph com (a) e sem (b) cadeias laterais, calculados via DFTB com $\omega=0.1 a_{0}^{-1}$. O módulo do isovalor é fixado em 0.015 .

Note que, de acordo com a figura acima, para ambos os orbitais a estrutura nodal das ligações não é afetada pelas cadeias laterais, conservando o grau de delocalização nas proximidades do pireno (centro do composto) e apresentando pequenas variações nos grupos aceitadores (extremidades do cromóforo).

O ganho computacional decorrente da redução do número de átomos possibilita a determinação do espectro de absorção a partir de um ensemble de 1000 configurações nucleares. Seguindo a abordagem introduzida na Seção 2.8, em cada uma das configurações o método LC-TD-DFTB/OB2+S é utilizado para determinar 50 estados excitados e as respectivas forças de oscilador. Lorentzianas são ajustadas em cada uma das excitações, de maneira análoga ao procedimento realizado com o código Lorentz, porém aqui o modelo de seção de choque de absorção (2.82) além de levar em 
consideração as constantes físicas associadas, envolve a média sobre configurações. O resultado desse modelo para o aceitador eletrônico na ausência de cadeias laterais (DTP-IC-4Ph ${ }^{\dagger}$ ) é apresentado na Figura 3.15, juntamente aos dados experimentais fornecidos por Wang et al. [15].

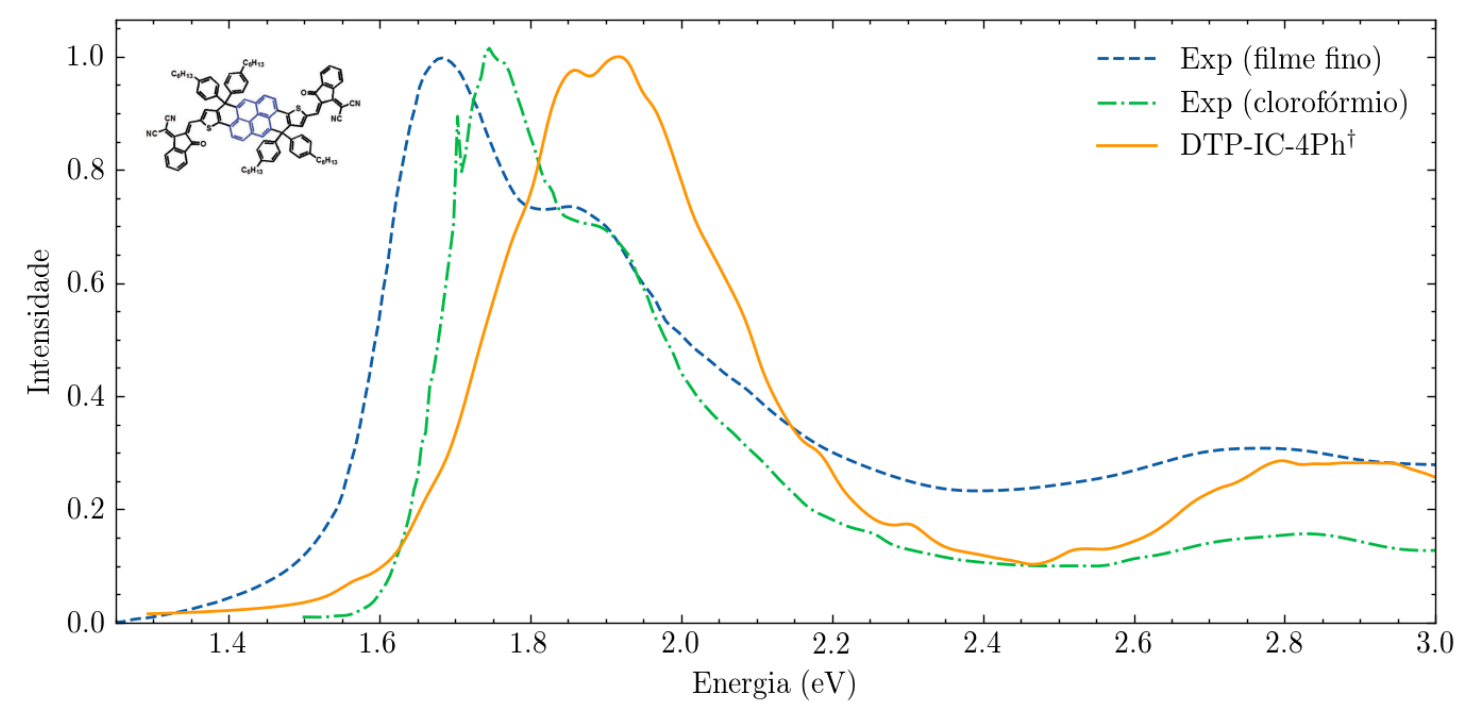

Figura 3.15: Espectros de absorção normalizados do composto DTP-IC-4Ph. As curvas tracejadas dizem respeito aos dados experimentais, obtidos por Wang et al. [15] a partir de um filme fino e de uma solução com clorofórmio $\left(\mathrm{CHCl}_{3}\right)$ como solvente, enquanto a curva sólida corresponde ao espectro, na ausência de cadeias laterais (identificadas como $\mathrm{C}_{6} \mathrm{H}_{13}$ na imagem do composto), determinado a partir de 1000 configurações via DFTB.

A linha tracejada azul traz os resultados experimentais medidos de uma película (cast film) do aceitador e a verde em solução de clorofórmio, enquanto a curva laranja é fruto dos cálculos teóricos realizados por meio da técnica NEA (Nuclear Ensemble Approach) implementada no código Newton-X [103], como descrito na Seção 2.8, para a molécula isolada. Para cada uma das configurações foram considerados 50 estados excitados que caracterizam a faixa de maior intensidade do espectro solar, desde o violeta $(\approx 400 \mathrm{~nm})$ até o início do infravermelho $(\approx 850 \mathrm{~nm})$.

Ainda que qualitativamente os cálculos via NEA $+D F T B$ reproduzam o comportamento do espectro, mantendo o pico na região do vermelho como observado experimentalmente, as curvas diferem (em menor proporção para o aceitador na presença do clorofórmio) tanto na energia do primeiro pico, quanto nas intensidades para maiores energias, com destaque para o platô em $1.8 \mathrm{eV}$, presente em ambos os espectros experimentais, que não aparece na curva laranja. Além das limitações teóricas, tais diferenças também devem ser associadas ao ambiente que envolve os compostos. Por um lado consideramos uma molécula isolada enquanto, experimentalmente, tem-se 
moléculas solvatadas e moléculas que compõem um filme fino. Embora em nenhum dos dois casos haja ordenamento como em cristais, a interação com a vizinhança traz efeitos que afetam o espectro e devem ser levados em consideração, reforçando a discussão iniciada na Subseção 3.2.2.

Ao comparar a curva laranja, resultado do ensemble de geometrias, com os espectros verticais da Figura 3.13, verificamos uma alteração clara nas intensidades relativas dos primeiros picos, como é apresentado na Figura 3.16 .
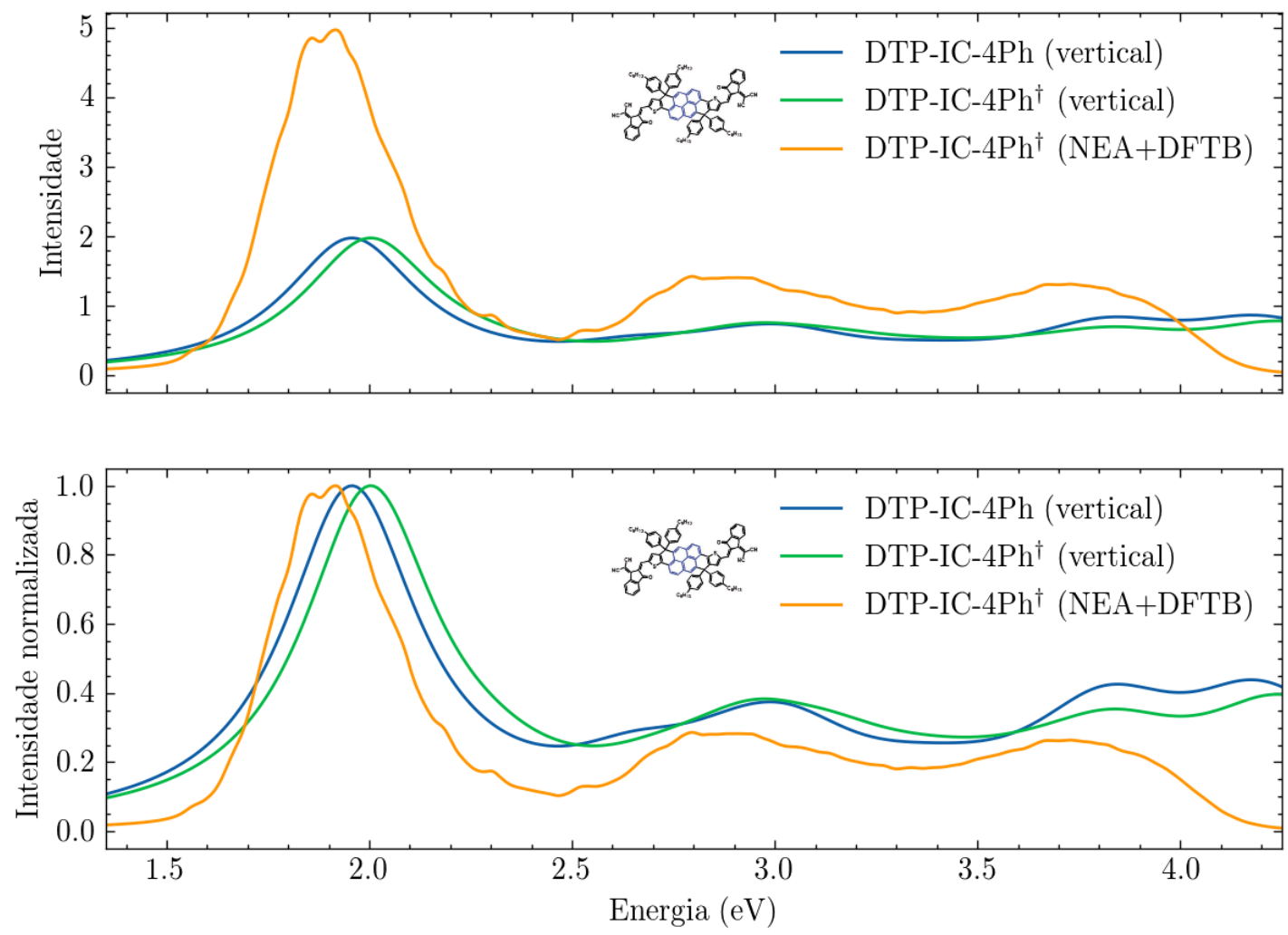

Figura 3.16: Espectros de absorção do composto DTP-IC-4Ph. No painel superior são apresentadas as intensidades absolutas e no inferior as intensidades normalizadas. As curvas verde e azul correspondem aos espectros verticais do aceitador com e sem cadeias laterais, respectivamente. A curva laranja corresponde ao espectro, na ausência de cadeias laterais, determinado a partir de 1000 configurações via DFTB. Todos os cálculos adotam o valor otimizado $\omega=0.1 a_{0}^{-1}$.

Ao observar o painel superior, é evidente que o primeiro pico passa a ser mais intenso quando consideramos a média sobre configurações, o que sugere que a absorção em torno de 2 eV está presente na maioria das configurações na região de Franck-Condon, e aponta que alterações nas geometrias são tais que preservam as forças de oscilador, ao menos para baixas energias. Os aumentos dos dois picos seguintes reforçam essa ideia e, em vista da regra de soma de Thomas-Reiche-Kuhn [104], 


$$
\sum_{n>0} f_{0 n}=N
$$

onde $f_{0 n}$ é a força de oscilador associada à transição do estado fundamental para o $n$-ésimo estado excitado e $N$ é o número de elétrons, resulta em espectros mais localizados em energia. As curvas normalizadas do painel inferior ilustram esse comportamento, uma vez que o espectro laranja se concentra na região de 1.5 até $4.0 \mathrm{eV}$ enquanto os verticais seguem para outras faixas de energia, como ilustrado na Figura 3.13. O deslocamento do espectro NEA+DFTB para o vermelho, associado às mudanças nos modos vibracionais do estado fundamental e dos estados excitados [105] também é observado. Por fim, o perfil irregular da curva reflete que o número de configurações não é suficiente para convergir a seção de choque, e tende a ser suavizado ao considerar um número maior de geometrias [82].

\subsubsection{PBDB-T}

No caso do doador, a sensibilidade do espectro de absorção frente a mudanças é mais significativa. A Figura 3.17 apresenta os espectros calculados na geometria do estado fundamental.

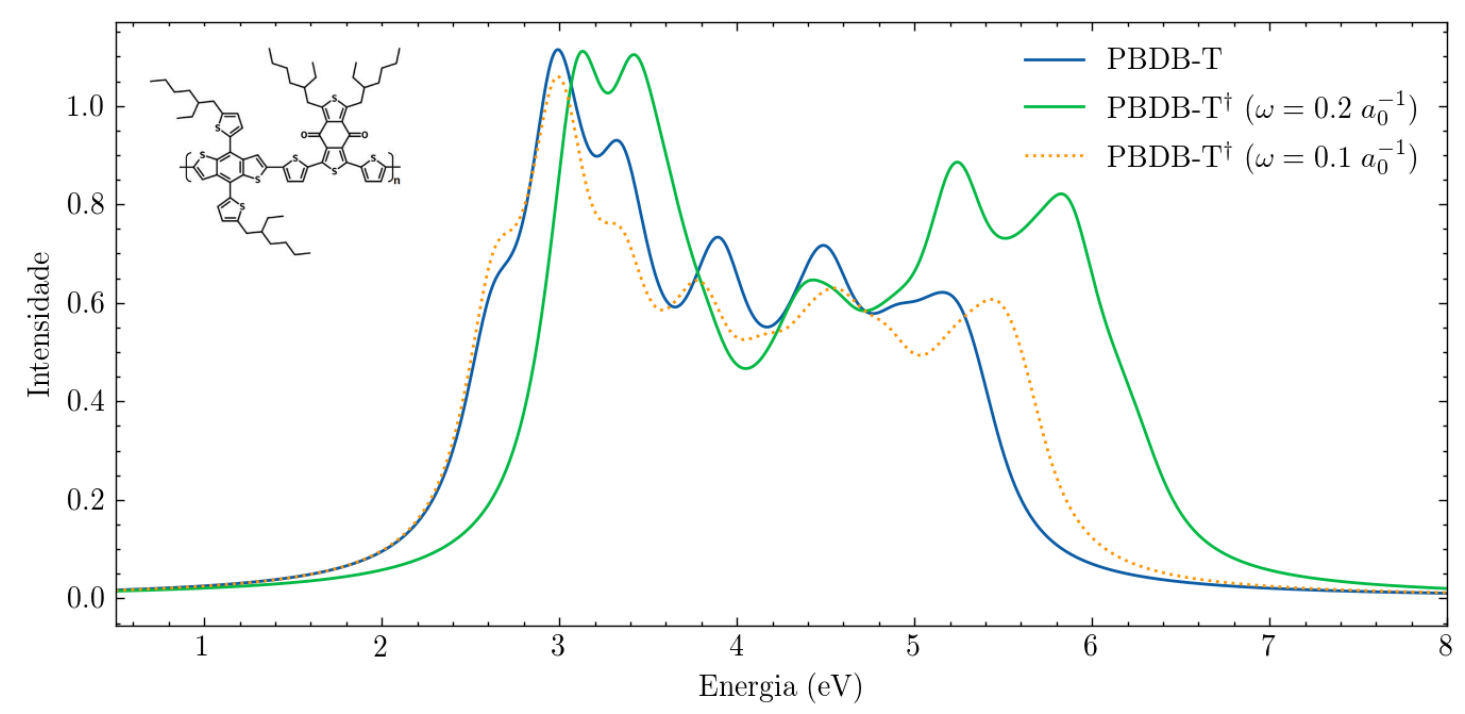

Figura 3.17: Espectros de absorção do composto PBDB-T. A curva azul descreve o monômero do composto $(\mathrm{n}=1)$, enquanto as demais curvas correspondem ao espectro na ausência de cadeias laterais. A linha verde utiliza o valor de $\omega$ otimizado na ausência de cadeias laterais $\left(\omega=0.2 a_{0}^{-1}\right.$ ), enquanto a pontilhada utiliza o valor otimizado para o composto na íntegra $\left(\omega=0.1 a_{0}^{-1}\right)$.

A curva azul corresponde ao espectro do monômero do PBDB-T $(\mathrm{n}=1)$ com as cadeias laterais, enquanto as outras duas curvas substituem as 4 
cadeias por átomos de hidrogênio, que nesse caso são da forma $\mathrm{C}_{8} \mathrm{H}_{17}$, ao invés de $\mathrm{C}_{6} \mathrm{H}_{13}$ como no aceitador eletrônico DTP-IC-4Ph. A linha verde considera $\omega=0.2 a_{0}^{-1}$, o valor otimizado para o composto na ausência das cadeias laterais, e as linhas azul e pontilhada adotam $\omega=0.1 a_{0}^{-1}$, o valor otimizado para a molécula com as cadeias. Quando comparamos as curvas com o mesmo parâmetro de longo alcance $\omega=0.1 a_{0}^{-1}$, observamos que a retirada das cadeias resulta na diminuição dos picos na faixa de 3 até $5 \mathrm{eV}$, o que é uma consequência direta da atenuação das forças de oscilador na região. Além do deslocamento rígido, característico da mudança de $\omega$, a curva verde acaba por intensificar algumas transições como a em torno de $3.4 \mathrm{eV}$ e amenizar outras, como observado em $4 \mathrm{eV}$.

Diferentemente do DTP-IC-4Ph, a retirada das cadeias acaba por impactar no formato do espectro como um todo. Tais mudanças não devem ser associadas ao caráter dos orbitais HOMO e LUMO, uma vez que eles não são afetados significativamente pela ausência dos segmentos $\mathrm{C}_{8} \mathrm{H}_{17}$ nem pela mudança de $\omega$, como é ilustrado na Figura 3.18. 


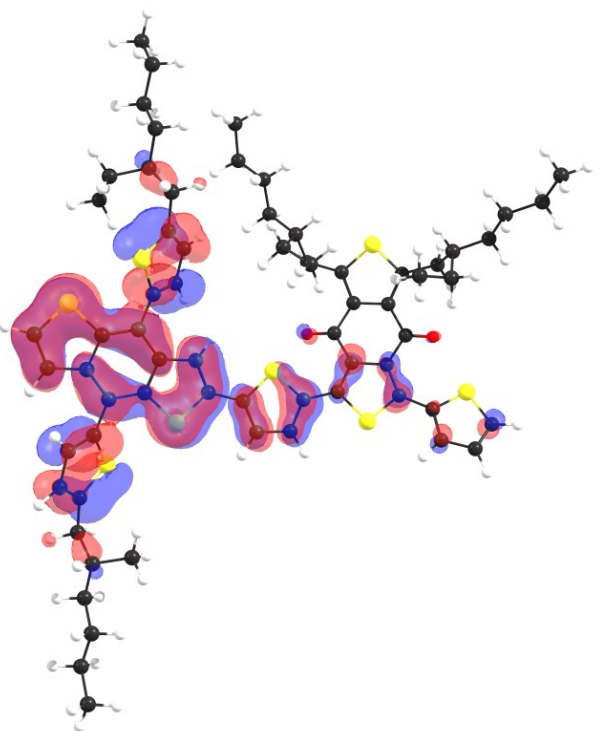

(a) (H) PBDB-T

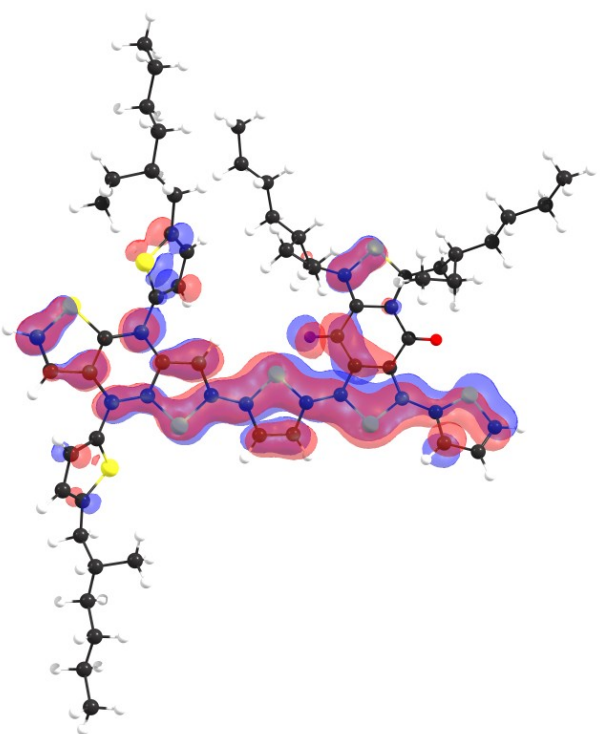

(d) (L) PBDB-T

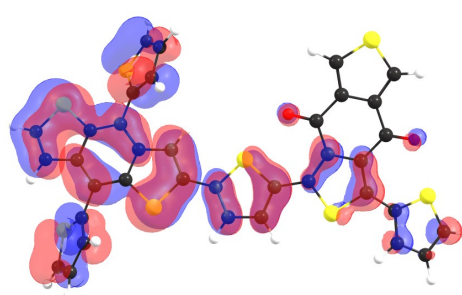

(b) $(\mathrm{H}) \mathrm{PBDB}^{\dagger} \mathrm{T}^{\dagger}$

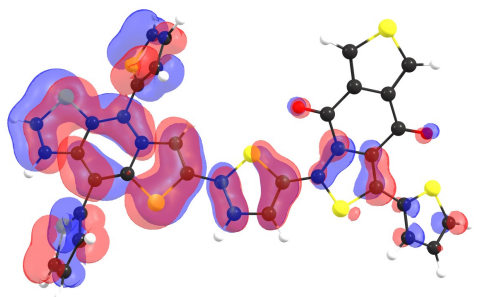

(c) $(\mathrm{H}) \mathrm{PBDB}^{\dagger}-\omega=0.2 a_{0}^{-1}$

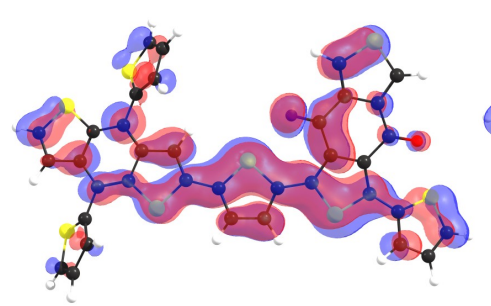

(e) (L) PBDB-T ${ }^{\dagger}$

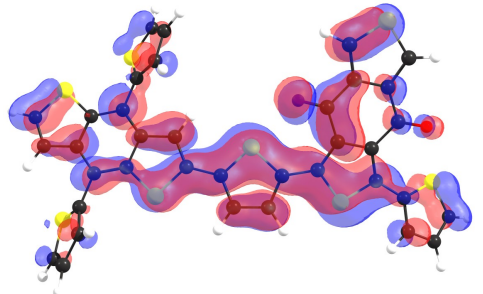

(f) (L) PBDB-T ${ }^{\dagger}-\omega=0.2 a_{0}^{-1}$

Figura 3.18: Isosuperfícies dos orbitais HOMO (primeira linha) e LUMO (segunda linha) do doador PBDB-T com e sem cadeias laterais, calculados via DFTB. As duas primeiras colunas utilizaram $\omega=0.1 a_{0}^{-1}$ e a terceira $\omega=0.2 a_{0}^{-1}$. O módulo do isovalor é fixado em 0.015 .

Além do impacto no parâmetro de longo alcance, a eliminação de 96 átomos acaba reduzindo a flexibilidade da molécula uma vez que as ligações simples que ligam os anéis de tiofeno $\left(\mathrm{C}_{4} \mathrm{H}_{4} \mathrm{~S}\right)$ estão sujeitas à rotações, resultando em geometrias distintas. De maneira análoga à Figura 3.15, a Figura 3.19 contrapõe os espectros de absorção calculados via Newton-X com o experimental. 


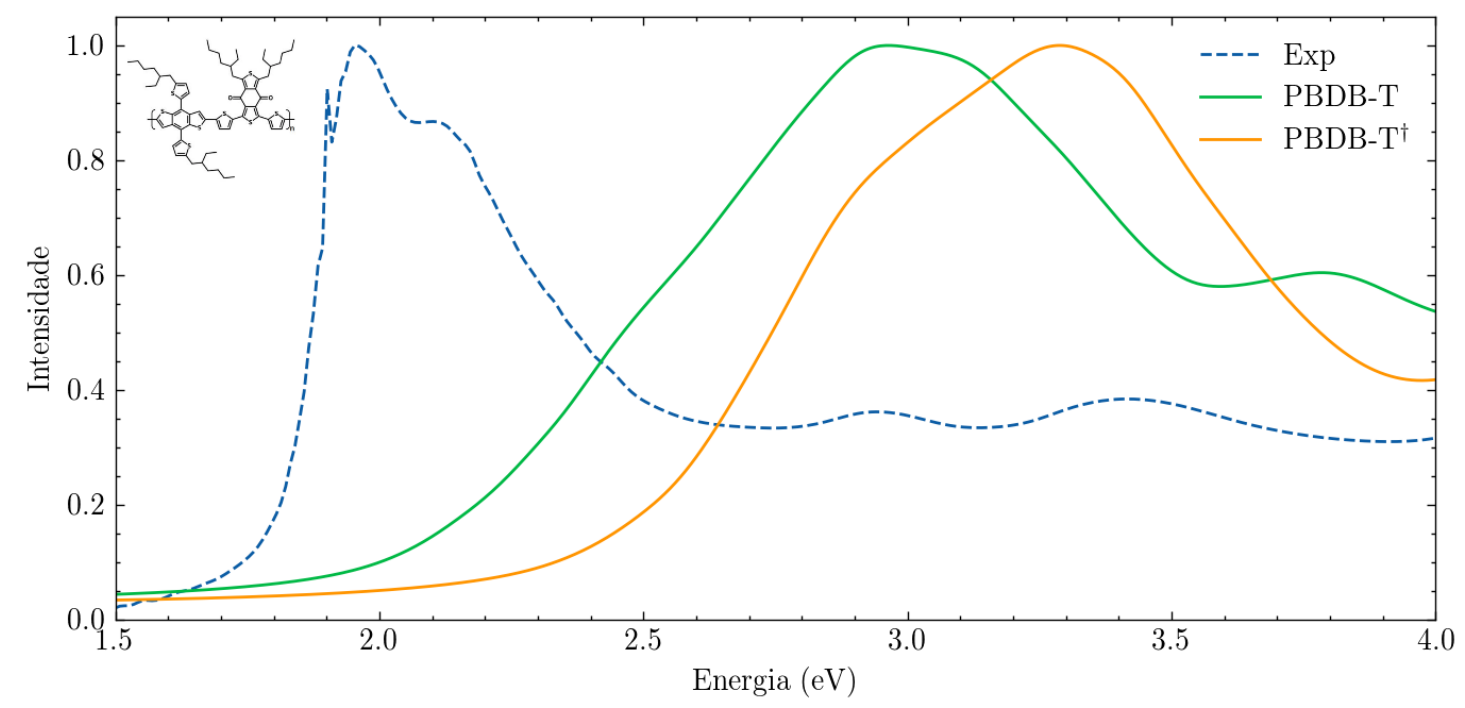

Figura 3.19: Espectros de absorção normalizados do PBDB-T. A linha tracejada diz respeito aos dados experimentais, fornecidos por Sunsun et al. [24], enquanto as linhas sólida correspondem aos espectros do monômero, determinados a partir de 1000 configurações via $D F T B$. A curva verde corresponde ao espectro do composto como representado na figura e a amarela na ausência das cadeias laterais.

A linha tracejada diz respeito ao espectro experimental do polímero de PBDB-T determinado por Sunsun et al. [24], enquanto as linhas sólidas foram calculadas a partir de 50 estados excitados oriundos de 1000 configurações do monômero, seguindo a mesma abordagem realizada para o composto aceitador. As curvas verde e amarela dizem respeito, respectivamente, ao monômero com e sem cadeias laterias, utilizando os correspondentes valores de $\omega$ otimizados.

Nesse cenário as discrepâncias são ainda mais evidentes. Experimentalmente, o pico de absorção se encontra na região do vermelho enquanto os cálculos apontam para o violeta e ultravioleta próximo. Ainda que a média sobre um conjunto de configurações diminua o efeito das cadeias laterais, observado na Figura 3.17, o alargamento do pico frente aos dados experimentais resulta em imprecisões na determinação de grandezas como a potência absorvida, exigindo um tratamento mais aprofundado do sistema. Um primeiro refinamento deve levar em conta que o doador é um polímero, e portanto o estudo de monômeros isolados é naturalmente limitado. Como discutido na Seção 3.2.3, o aumento do número de oligômeros impacta significativamente a energia dos orbitais [41], e portanto o espectro de absorção.

Por completeza, vale destacar que o ensemble de geometrias (curvas sólidas 
da Figura 3.19) produz o mesmo efeito observado para o DTP-IC-4Ph, ao comparar com os espectros verticais da Figura 3.17. Assim como na Figura 3.16, há uma tendência de estreitamento da faixa de absorção e um aumento nos picos de absorção, porém sem a expressividade do primeiro pico do aceitador eletrônico.

\subsection{Estudo do efeito de solvente em HPAs}

Em vista da importância dos efeitos do ambiente na caracterização dos compostos, uma tentativa de quantificá-los é realizada por meio de modelos contínuos de solvatação. Como tais modelos não estão implementados no código DFTB+, impossibilitando o estudo do aceitador e do doador eletrônico, foram realizados cálculos para os HPAs em nível (TD)DFT/ $\omega$ B97X-D/6-31G(d,p) com o modelo contínuo polarizável (PCM), através do pacote computacional Gaussian09 [88].

Os hidrocarbonetos pertencem ao grupo pontual de simetria $D_{2 h}$ e, devido à sua composição puramente formada por carbonos e hidrogênios, possuem caráter apolar. Entre as opções de solventes o clorofórmio $\left(\varepsilon_{\mathrm{CHCl}_{3}}=4.7\right)$ e a água $\left(\varepsilon_{\mathrm{H}_{2} \mathrm{O}}=78.4\right)$ foram selecionados. O primeiro, usualmente utilizado no estudo de compostos orgânicos, foi escolhido em decorrência de resultados experimentais em solução de clorofórmio do espectro de absorção dos compostos produzidos por Wang et al. [15], e a água como forma de investigar o impacto de um solvente mais fortemente polar.

Ainda que os solventes possuam propriedades distintas, as otimizações resultaram em geometrias essencialmente iguais, com variações da ordem de $10^{-3} \AA$ nos comprimentos de ligação e $10^{-3}$ graus para os ângulos entre ligações atômicas e ângulos de torsão, exemplificadas pela raiz do desvio quadrático médio apresentada na Tabela 3.14.

\begin{tabular}{c|ccc} 
& \multicolumn{3}{|c}{$\operatorname{RMSD}(\AA)$} \\
\hline & Naftaleno & Antraceno & Pireno \\
\hline Água $\left(\mathrm{H}_{2} \mathrm{O}\right)$ & 0.0019 & 0.0017 & 0.0018 \\
Clorofórmio $\left(\mathrm{CHCl}_{3}\right)$ & 0.0010 & 0.0011 & 0.0012 \\
\hline
\end{tabular}

Tabela 3.14: Raízes do desvio quadrático médio (RMSD) dos comprimentos de ligação em relação a geometria otimizada via DFT sem PCM, para as moléculas de naftaleno e pireno. 
As alterações nas cargas parciais atômicas são iguais para os dois solventes, o que sugere uma saturação na polarização do soluto, uma vez que o aumento da constante dielétrica de 4.7 (clorofórmio) para 78.4 (água) não impacta na distribuição de cargas. O mesmo efeito não deve ser observado ao solvatar os compostos maiores (doador/aceitador eletrônico), uma vez que eles pertencem ao grupo de simetria pontual $C_{1}$ e possuem grupos polares em suas composições.

\begin{tabular}{c|cc|cc|cc} 
& \multicolumn{2}{|c|}{ Vácuo } & \multicolumn{2}{c|}{ Água $\left(\mathbf{H}_{2} \mathbf{O}\right)$} & \multicolumn{2}{c}{ Clorofórmio $\left(\mathbf{C H C l}_{3}\right)$} \\
\hline & $\epsilon_{\text {HOMO }}(\mathrm{eV})$ & $E_{\text {gap }}^{H-L}(\mathrm{eV})$ & $\epsilon_{\text {HOMO }}(\mathrm{eV})$ & $E_{\text {gap }}^{H-L}(\mathrm{eV})$ & $\epsilon_{\text {HOMO }}(\mathrm{eV})$ & $E_{\text {gap }}^{H-L}(\mathrm{eV})$ \\
\hline Naftaleno & -7.748 & 8.578 & -7.892 & 8.579 & -7.839 & 8.579 \\
Antraceno & -7.081 & 7.136 & -7.230 & 7.227 & -7.173 & 7.210 \\
Pireno & -7.131 & 7.289 & -7.289 & 7.290 & -7.228 & 7.290 \\
\hline
\end{tabular}

Tabela 3.15: Energias dos orbitais HOMO $\left(\epsilon_{\text {HOMO }}\right)$ e as diferenças entre HOMO e LUMO, $E_{\text {gap }}^{H-L}$, calculadas para os HPAs solvatados em clorofórmio e água a nível PCM.

A Tabela 3.15 traz, para cada composto, as energias do orbital HOMO e do gap. Ainda que mais significativas do que para as geometrias, as variações nas energias dos orbitais permanecem pequenas e são tais que os gaps se mantêm aproximadamente constantes mesmo na presença de solventes, retratando um deslocamento rígido na energia dos orbitais de fronteira. O caráter dos mesmos permanece inalterado, e em concordância com o esperado para compostos aromáticos, tanto o LUMO quanto o HOMO, são orbitais do tipo $\pi$. A figura abaixo ilustra os orbitais do pireno, que são semelhantes aos apresentados na Figura 3.1.
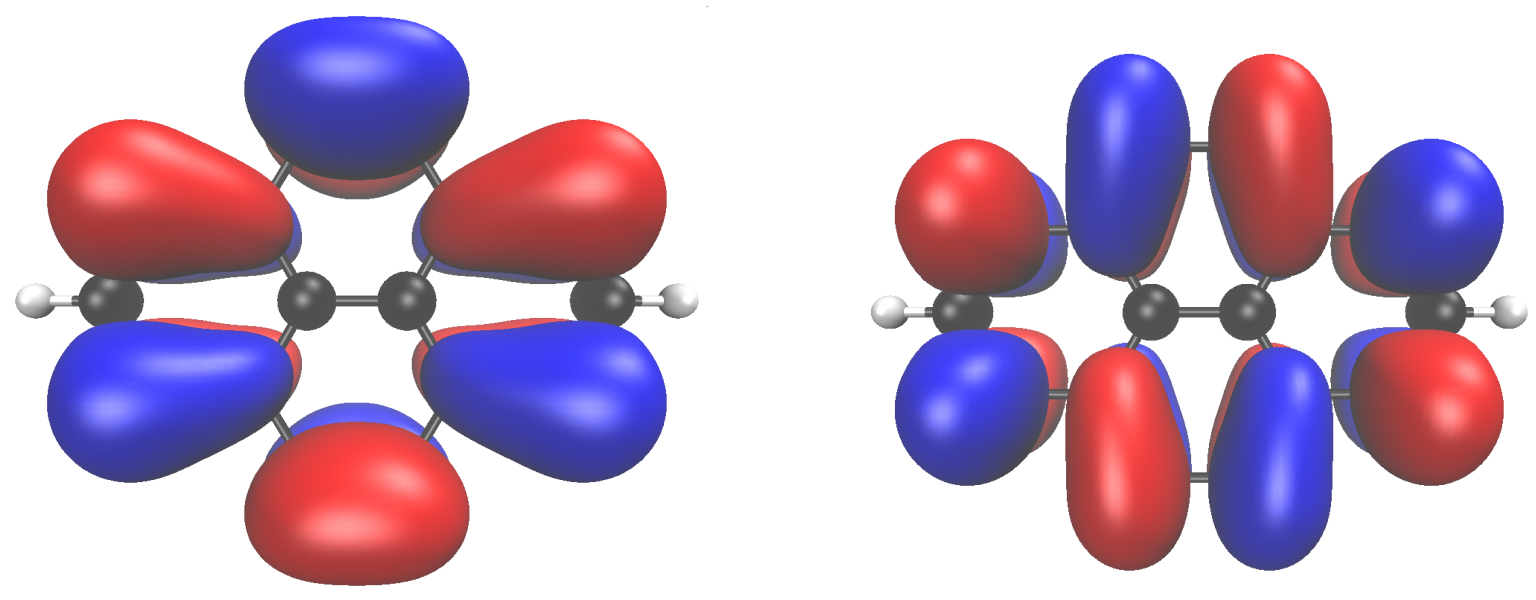

Figura 3.20: Orbitais HOMO (à esquerda) e LUMO (à direita) da molécula de pireno otimizada a nível $\omega$ B97X-D/6-31G(d,p)/PCM com clorofórmio como solvente.

Para os demais orbitais o efeito dos solventes nas energias é semelhante e 
gera um deslocamento rígido no espectro de absorção, observado na Figura 3.21 , que apresenta os espectros dos três hidrocarbonetos no vácuo e na presença dos solventes água e clorofórmio.
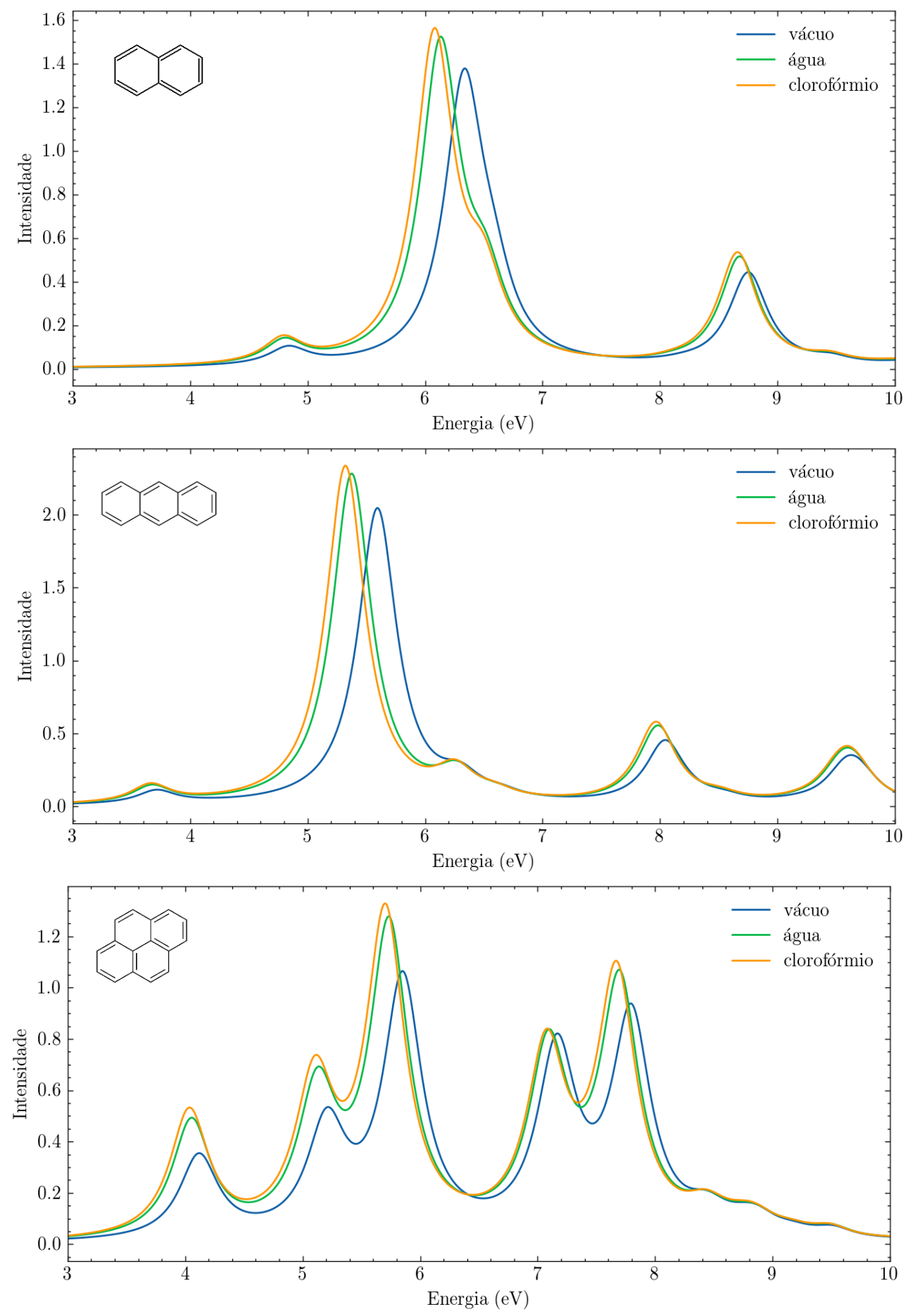

Figura 3.21: Espectros de absorção dos compostos HPAs à vácuo e solvatados com água $\left(\mathrm{H}_{2} \mathrm{O}\right)$ e clorofórmio $\left(\mathrm{CHCl}_{3}\right)$. Os cálculos foram realizados através do software Gaussian09 [88] em nível TD-DFT/wB97X-D/6-31G(d,p) com PCM, envolvendo 100 estados excitados.

Os deslocamentos dos picos de absorção são maiores nas regiões de menor 
energia e apresentam valores inferiores a $0.2 \mathrm{eV}$, com destaque para o pico em $5.85 \mathrm{eV}$ do pireno que é estabilizado em $5.70 \mathrm{eV}$ na presença de clorofórmio como solvente.

O efeito de solvatocronismo neste contexto é caracterizado pelo red shift do espectro durante a mudança de fase gasosa para líquida, o que tende a compensar o blue shift do espectro teórico em relação ao experimental, observado nas Figura 3.15 e 3.19. Por se tratar de solutos apolares, os momentos de dipolo permanentes são nulos e a interação com a vizinhança é determinada pelos momentos de quadrupolo permanentes e pelas forças de dispersão decorrentes dos momentos de dipolo induzidos (forças de London). Do ponto de vista das interações de dipolo induzido, em ambos os casos é esperada uma dependência diretamente proporcional entre o red shift e o índice de refração $n$ do solvente [106], que é de fato observada uma vez que $n_{\mathrm{H}_{2} \mathrm{O}}=1.33$ e $n_{\mathrm{CHCl}_{3}}=1.45$. A polarizabilidade que determina a força da interação de London, aumenta durante excitações do tipo $\pi \rightarrow \pi^{*}$ pois o orbital $\pi^{*}$ está mais distante da estrutura nuclear atrativa, ${ }^{10}$ e portanto o estado excitado desfruta de uma interação de dispersão mais forte com o solvente circundante do que o estado fundamental menos polarizável. Em adição, a interação entre o quadrupolo e o dielétrico é maior entre o estado fundamental e estados excitados de menor energia [108], contribuindo para maiores deslocamentos em regiões de baixa energia, como observado nas bandas de absorção da Figura 3.21.

\subsection{Modelos para Células Solares}

Tendo em vista o interesse em aplicar a metodologia LC-TD-DFTB à modelagem de células solares, além da caracterização da estrutura eletrônica e do espectro de absorção dos compostos que compõem a camada ativa, algumas grandezas relevantes foram calculadas, i.e., constantes dielétricas $(\epsilon)$, energias de ligação do éxciton $\left(E_{b}\right)$ e energias intramoleculares de reorganização $(\lambda)$, e os resultados são apresentados nessa seção.

As constantes dielétricas caracterizam propriedades eletromagnéticas dos materiais, sendo mediadoras da interação eletrostática via PCM. Energias de ligação dos éxcitons quantificam a interação do par elétron-buraco, en-

\footnotetext{
${ }^{10}$ Como exemplo, para o composto de benzeno a componente $z z$ da polarizabilidade aumenta de 44 para 52 unidades atômicas [107].
} 
quanto as energias intramoleculares de reorganização compõem o modelo de mobilidade dos portadores de carga, discutido na Subseção 3.6.3.

\subsubsection{Constantes dielétricas}

O cálculo de constantes dielétricas é realizado por meio da relação de Clausius-Mossotti, apresentada na Seção 2.7. A equação (2.80) determina $\epsilon$ a partir da polarizabilidade $(\alpha)$ do composto,

$$
\frac{\epsilon-1}{\epsilon-2}=\frac{4 \pi}{3} \frac{\alpha}{V},
$$

sendo $V$ o volume da molécula. A polarizabilidade total possui três contribuições, de acordo com a equação (2.81). No caso de moléculas, o termo iônico é desprezível e a polarizabilidade eletrônica é dada pelo valor médio,

$$
\alpha_{0} \equiv\left\langle\alpha_{e}\right\rangle=\frac{\operatorname{Tr}(\boldsymbol{\alpha})}{3}=\frac{\alpha_{x x}+\alpha_{y y}+\alpha_{z z}}{3},
$$

sendo $\boldsymbol{\alpha}$ o tensor de polarizabilidade calculado a partir da resposta da molécula frente a um campo elétrico estático, por intermédio da teoria de perturbação (ver Apêndice N). Essa polarizabilidade média também é chamada de polarizabilidade isotrópica $\left(\alpha_{0}\right)$ devido a sua invariância frente à direção do campo elétrico. A Tabela 3.16 traz os valores de $V, \alpha_{0}$ e do módulo do momento de dipolo permanente $(\mu)$ para os compostos de interesse. 


\begin{tabular}{|c|c|c|c|c|c|c|}
\hline & \multicolumn{3}{|c|}{$D F T$} & \multicolumn{3}{|c|}{$D F T B$} \\
\hline Sistema & $V\left(\AA^{3}\right)$ & $\alpha_{0}\left(\AA^{3}\right)$ & $\mu$ (u.a.) & $V\left(\AA^{3}\right)$ & $\alpha_{0}\left(\AA^{3}\right)$ & $\mu$ (u.a.) \\
\hline Naftaleno & 179.23 & 14.49 & $1.4 \times 10^{-3}$ & 161.62 & 11.32 & $1.5 \times 10^{-5}$ \\
\hline Antraceno & 218.78 & 22.35 & $1.0 \times 10^{-4}$ & 215.30 & 17.97 & $6.2 \times 10^{-5}$ \\
\hline Pireno & 250.81 & 24.68 & $1.0 \times 10^{-4}$ & 234.20 & 20.14 & $1.2 \times 10^{-4}$ \\
\hline DTP-IC-4Ph & - & - & - & 1677.90 & 333.25 & 1.60 \\
\hline DTP-IC-4Ph ${ }^{\dagger}$ & - & - & - & 1157.91 & 244.31 & 1.23 \\
\hline PBDB-T & - & - & - & 1357.47 & 174.99 & 1.05 \\
\hline $\mathrm{PBDB} \mathrm{T}^{\dagger}$ & - & - & - & 709.73 & 78.47 & 0.31 \\
\hline
\end{tabular}

Tabela 3.16: Volume $(V)$, polarizabilidade isotrópica $\left(\alpha_{0}\right)$ e módulo do momento de dipolo permanente $(\mu)$ dos compostos naftaleno, antraceno, pireno, DTP-IC-4Ph e do monômero de PBDB-T, calculados com ambos os métodos. As linhas PBDB-T ${ }^{\dagger}$ e DTP-IC-4Ph ${ }^{\dagger}$ dizem respeito aos valores obtidos na ausência de cadeias laterais.

Os cálculos de geometria e polarizabilidade dos HPAs foram realizados utilizando ambos os métodos, seguindo com a validação da $D F T B .{ }^{11}$ De acordo com a tabela, as polarizabilidades em nível DFTB são no geral subestimadas em $25 \%$ com relação as $D F T$, o que tende a diminuir o valor de $\epsilon$, uma vez a constante dielétrica é diretamente proporcional à polarizabilidade. Devido à simetria do grupo $D_{2 h}$, os momentos de dipolo permanente dos três compostos são nulos, o que é bem representado em ambos os formalismos.

Há diferença relevante na determinação dos volumes moleculares por meio de cada metodologia. Em ambos os casos é utilizado o método de Monte Carlo, mas para DFT, a região delimitada pela molécula é fixada como sendo aquela na qual a densidade eletrônica é maior que 0.001 elétrons $/ \mathrm{bohr}^{3}$, enquanto na DFTB foram utilizados raios de van der Waals obtidos via DFT-D3 [110] para determinar os volumes molecular a partir de esferas centradas em cada átomo. ${ }^{12}$ Ainda assim os resultados foram próximos, a DFTB fornece volumes menores, o que acaba por aumentar o valor de $\epsilon$, podendo vir a compensar a diminuição decorrente da redução de $\alpha_{0}$. O cálculo dos volumes é discutido com mais detalhes no Apêndice $\mathrm{O}$, bus-

\footnotetext{
${ }^{11}$ Cálculos equivalentes utilizando o Gaussian03 com DFT/B3LYP/6-31G(d) forneceram resultados promissores para estruturas HPAs [109], o que sugere que o método DFT/ $\omega$ B97X-D/6-31G(d,p) deve fornecer bons resultados.

${ }^{12} \mathrm{~A}$ escolha destes raios de van der Waals é devido a utilização dos mesmos pelo software DFTB+ (https://dftbplus.org/fileadmin/DFTBPLUS/public/dftbplus/latest/manual.pdf).
} 
cando validar o algoritmo desenvolvido. A partir dos dados apresentados na tabela anterior, a Tabela 3.17 confronta as constantes dielétrica calculadas com as experimentais.

\begin{tabular}{c|c|c|c} 
& DFT & DFTB & Experimental \\
\hline Sistema & \multicolumn{2}{|c}{$\epsilon$} \\
\hline Naftaleno & 2.54 & 2.25 & 2.87 \\
Antraceno & 3.24 & 2.61 & 3.12 \\
Pireno & 3.10 & 2.69 & 3.14 \\
DTP-IC-4Ph & - & 15.85 & - \\
DTP-IC-4Ph $^{\dagger}$ & - & 23.82 & - \\
PBDB-T $^{-}$ & - & 4.52 & 2.80 \\
PBDB-T $^{\dagger}$ & - & 3.59 & - \\
\hline
\end{tabular}

Tabela 3.17: Constantes dielétricas $(\epsilon)$ dos compostos naftaleno, antraceno, pireno, DTPIC-4Ph e do monômero de PBDB-T, obtidos com ambos os métodos e experimentalmente. As linhas $\mathrm{PBDB}^{\dagger} \mathrm{T}^{\dagger}$ e DTP-IC-4Ph ${ }^{\dagger}$ dizem respeito aos valores obtidos na ausência de cadeias laterais.

Os volumes calculados com DFT se aproximam mais dos experimentais. Tal comportamento pode ser atribuído às menores polarizabilidades calculadas via DFTB que acabam por reduzir as constantes dielétricas. Essas diferenças em $\alpha_{0}$ apontam que, uma vez que as forças de oscilador são próximas para as duas metodologias, nos cálculos DFT a densidade de estados excitados nas vizinhanças do estado fundamental, ou seja, estados de baixa energia, deve ser maior (ver Apêndice N), o que pode ser observado nos espectros de absorção apresentados nas Figuras 3.10, 3.11 e 3.12. As curvas pontilhadas se encontram mais próximas da origem, e portanto em média, as diferenças de energia entre os estados excitados e o estado fundamental $\left(E_{n}-E_{0}\right)$ são menores, resultando em polarizabilidades isotrópicas maiores, de acordo com a equação (N.3).

Comparando o valor experimental com os cálculos para o monômero de PBDB-T, observamos um comportamento oposto ao dos hidrocarbonetos, superestimando a constante dielétrica. Além da possibilidade de que os raios de van-der-Waals resultem em volumes inferiores, é de se esperar que diante da presença de outros oligômeros o espectro de absorção sofra um blue shift, sendo deslocado para energias inferiores e portanto, resultando no aumento de $\alpha_{0}$, e consequentemente, no aumento de $\epsilon$. Outra 
disparidade é verificada quando removemos as cadeias laterais, o valor de $\epsilon$ aumenta para o DTP-IC-4Ph e diminui para o PBDB-T. Essa diminuição pode ser atribuída à mudança do parâmetro ótimo de longo alcance, de $0.1 a_{0}^{-1}$ para $0.2 a_{0}^{-1}$, que como podemos observar na Figura 3.19, também realiza um blue shift no espectro de absorção.

Uma melhor estimativa para as constantes dielétricas é obtida por meio de uma regressão linear aos dados experimentais [111]. A partir do Método de Mínimos Quadrados [112], para cada composto do "conjunto de treinamento", as constantes dielétricas experimentais são expressas no eixo vertical em função das teóricas no eixo horizontal e uma reta é ajustada. De posse dos coeficientes angular e linear as novas constantes dielétricas são determinadas utilizando os valores da coluna DFTB da Tabela 3.17. Os resultados obtidos a partir do ajuste linear que contempla as moléculas de benzeno $\left(\mathrm{C}_{6} \mathrm{H}_{6}\right)$, naftaleno, antraceno, pireno e tiofeno $\left(\mathrm{C}_{4} \mathrm{H}_{4} \mathrm{~S}\right)$ como conjunto de treinamento são comparados com os dados experimentais na Tabela 3.18.

\begin{tabular}{c|c|c} 
& Ajuste & Experimental \\
\hline Sistema & \multicolumn{2}{|c}{$\epsilon$} \\
\hline Naftaleno & 2.83 & 2.87 \\
Antraceno & 3.09 & 3.12 \\
Pireno & 3.15 & 3.14 \\
DTP-IC-4Ph & 12.75 & - \\
DTP-IC-4Ph $^{\dagger}$ & 18.56 & - \\
PBDB-T $^{-1}$ & 4.49 & 2.80 \\
PBDB-T $^{\dagger}$ & 3.15 & - \\
\hline
\end{tabular}

Tabela 3.18: Constantes dielétricas $(\epsilon)$ dos compostos naftaleno, antraceno, pireno, DTPIC-4Ph e do monômero de PBDB-T, obtidas experimentalmente e via ajuste. As linhas $\mathrm{PBDB} \mathrm{T}^{\dagger}$ e DTP-IC-4Ph ${ }^{\dagger}$ dizem respeito aos valores obtidos na ausência de cadeias laterais.

O ajuste amplia a convergência de todos os resultados com os dados experimentais, com destaque já esperado, para os hidrocarbonetos que foram utilizados durante a regressão. 


\subsubsection{Energia de ligação do éxciton}

Como foi discutido previamente, um dos fatores responsáveis pela eficiência das células solares é a atração coloumbiana que tende a estabilizar o éxciton, impedindo que os portadores de carga se separem. Essa interação é chamada energia de ligação do éxciton $\left(E_{b}\right)$ e auxilia na caracterização da quasipartícula. Para semicondutores com baixas constantes dielétricas, são formados éxcitons de Frenkel, com energias de ligação no intervalo de 0.1 até $1 \mathrm{eV}$. Quando as contantes dielétricas são elevadas, como no caso de compostos a base de silício, as energias de ligação da ordem $0.01 \mathrm{eV}$ formam éxcitons mais extensos chamados de Wannier-Mott, o que é vantajoso pois favorece a separação do par buraco-elétron.

No caso de semicondutores orgânicos, baixas constantes dielétricas levam à criação de éxcitons moleculares com caráter fortemente ligado que competem com processos de recombinação. Sobrevivendo frente aos decaimentos radiativos e não radiativos, o elétron excitado tende a ocupar um orbital do aceitador eletrônico, caracterizando a formação de um estado de transferência de carga (CT) responsável pela melhoria no desempenho do dispositivo fotovoltaico. O cálculo da energia de ligação é determinado pela diferença [113],

$$
E_{b}=E_{\text {gap }}^{\mathrm{H}-\mathrm{L}}-E_{\text {gap }}^{\mathrm{opt}},
$$

onde $E_{\text {gap }}^{\mathrm{H}-\mathrm{L}}$ é a diferença entre o potencial de ionização (IP) e a afinidade eletrônica (EA), calculados a partir das diferenças de energia entre os íons

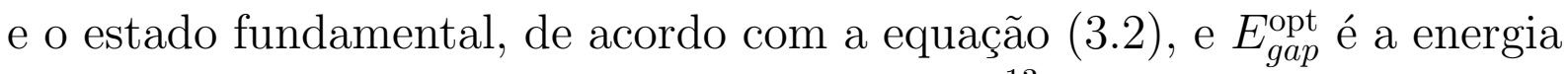
do primeiro estado excitado oticamente ativo. ${ }^{13}$ A Tabela 3.19 traz as energias calculadas para as moléculas isoladas de interesse.

\footnotetext{
${ }^{13}$ É importante ressaltar que o primeiro estado excitado oticamente ativo não é necessariamente o primeiro estado brilhante, o que fica claro ao comparar $S_{1}$ com $S_{B 1}$ na Tabela M.1.
} 


\begin{tabular}{|c|c|c|c|c|}
\hline & \multicolumn{2}{|c|}{$D F T$} & \multicolumn{2}{|c|}{$D F T B$} \\
\hline Sistema & $\omega\left(a_{0}^{-1}\right)$ & $E_{b}(\mathrm{eV})$ & $\omega\left(a_{0}^{-1}\right)$ & $E_{b}(\mathrm{eV})$ \\
\hline \multirow{2}{*}{ Naftaleno } & \multirow{2}{*}{0.2} & \multirow{2}{*}{4.09} & 0.3 & 4.42 \\
\hline & & & 0.5 & 4.39 \\
\hline \multirow{2}{*}{ Antraceno } & \multirow{2}{*}{0.2} & \multirow{2}{*}{3.44} & 0.3 & 3.74 \\
\hline & & & 0.5 & 3.69 \\
\hline \multirow{2}{*}{ Pireno } & \multirow{2}{*}{0.2} & \multirow{2}{*}{3.19} & 0.3 & 3.51 \\
\hline & & & 0.5 & 3.48 \\
\hline DTP-IC-4Ph & - & - & 0.1 & 0.95 \\
\hline DTP-IC-4Ph ${ }^{\dagger}$ & - & - & 0.1 & 1.34 \\
\hline PBDB-T & - & - & 0.1 & 1.45 \\
\hline \multirow{2}{*}{$\mathrm{PBDB}_{-} \mathrm{T}^{\dagger}$} & - & - & 0.1 & 2.18 \\
\hline & - & - & 0.2 & 2.33 \\
\hline
\end{tabular}

Tabela 3.19: Energias de ligação do éxciton $\left(E_{b}\right)$ dos compostos naftaleno, antraceno e pireno, DTP-IC-4Ph e monômero de PBDB-T calculadas utilizando ambas os métodos. As linhas $\mathrm{PBDB}-\mathrm{T}^{\dagger}$ e DTP-IC-4Ph${ }^{\dagger}$ dizem respeito aos valores obtidos na ausência de cadeias laterais.

Assim como nas diferenças HOMO-LUMO, os cálculos dos hidrocarbonetos apontam que no método DFTB a mudança do parâmetro de longo alcance diminui levemente a energia e, quando comparamos com o DFT, os resultados como um todo tendem a superestimar $E_{b}$. Ainda que é razoável esperar alterações na energia de ligação dos éxcitons ao considerar mais de um oligômero de PBDB-T, os baixos valores corroboram sua utilização em células solares e obedecem a relação inversamente proporcional entre $\epsilon$ e $E_{b}$ (ver Tabela 3.18). Uma constatação inusitada diz respeito à redução da energia de ligação na presença das cadeias laterais, que a priori atuam exclusivamente na melhoria da solubilidade dos compostos. Os valores do PBDB-T para $\omega=0.1$ e $0.2 a_{0}^{-1}$ indicam que a redução não é fruto da mudança do parâmetro ótimo e sim uma consequência da remoção dos radicais.

\subsubsection{Energia intramolecular de reorganização}

Para compreender as propriedades de transporte dos materiais, a mobilidade (hopping mobility) dos portadores de carga entre moléculas adjacentes 
$\left(k_{i}\right)$ pode ser determinada experimentalmente utilizando o método SCLC (Space Charge-Limited Current)[15]. Ainda que a modelagem teórica de $k$ seja complexa, o limite superior é tipicamente calculado a partir da teoria semiclássica de Marcus, dada por,

$$
k_{i}=|V|^{2} \sqrt{\frac{\pi}{\lambda_{i} \hbar^{2} k_{B} T}} e^{-\frac{\left(\lambda_{i}+\Delta G_{0}\right)^{2}}{4 \lambda_{i} k_{B} T}},
$$

onde $i$ diz respeito ao elétron ou buraco, $V$ é o acoplamento eletrônico entre o estado excitado e o estado de transferência de carga (CT), $\Delta G_{0}$ é a variação de energia livre também chamada de força motriz e $\lambda_{b / e}$ a energia intramolecular de reorganização do buraco/elétron. A força motriz pode ser determinada a partir da diferença de energia entre os estados inicial e final, e a determinação dos acoplamentos eletrônicos envolvem o cálculo de integrais de transferência de carga entre dímeros [80], que não foram explorado no presente trabalho. As demais variáveis da equação (3.3) dizem respeito à temperatura $T$ e à energia intramolecular de reorganização $\lambda$, entendida como a energia associada à mudança nas coordenadas nucleares devido à movimentação dos portadores de carga [19]. Essa energia pode ser decomposta em duas parcelas,

$$
\lambda=\lambda_{\text {in }}+\lambda_{\text {sol }},
$$

sendo $\lambda_{\text {in }}$ a contribuição interna (energia vibracional de reorganização) da molécula e $\lambda_{\text {sol }}$ a contribuição externa do solvente. Desprezando em um primeiro momento as contribuições do solvente, o modelo de Nelsen propõe que [114],

$$
\lambda_{i n}^{b / e}=\left(E_{0}^{ \pm}-E_{ \pm}\right)+\left(E_{ \pm}^{0}-E_{0}\right),
$$

onde $E_{0}^{+}\left(E_{0}^{-}\right)$é a energia do cátion(ânion) calculada na geometria do estado fundamental, $E_{+}\left(E_{-}\right)$a energia do cátion(ânion) calculada na geometria otimizada do próprio cátion(ânion), $E_{0}^{+}\left(E_{0}^{-}\right)$a energia da molécula neutra calculada na geometria otimizada do cátion(ânion) e $E_{0}$ a energia do estado fundamental da molécula neutra. Os valores calculados para os HPAs por meio da equação (3.4) são apresentados na Tabela 3.20. 


\begin{tabular}{c|ccc|ccc} 
& \multicolumn{3}{|c|}{ DF } & \multicolumn{3}{c}{ DF TB } \\
\hline Sistema & $\omega\left(a_{0}^{-1}\right)$ & $\lambda_{i n}^{b}(\mathrm{eV})$ & $\lambda_{i n}^{e}(\mathrm{eV})$ & $\omega\left(a_{0}^{-1}\right)$ & $\lambda_{i n}^{b}(\mathrm{eV})$ & $\lambda_{i n}^{e}(\mathrm{eV})$ \\
\hline \multirow{2}{*}{ Naftaleno } & 0.2 & 0.240 & 0.315 & 0.3 & 0.233 & 0.551 \\
& & & & 0.5 & 0.275 & 0.624 \\
\hline \multirow{2}{*}{ Antraceno } & 0.2 & 0.198 & 0.259 & 0.3 & 0.184 & 0.379 \\
\hline \multirow{2}{*}{ Pireno } & 0.2 & 0.232 & 0.295 & 0.3 & 0.223 & 0.446 \\
\hline
\end{tabular}

Tabela 3.20: Energias intramoleculares de reorganização internas dos buracos e elétrons $\left(\lambda_{i n}^{b / e}\right)$ dos compostos naftaleno, antraceno e pireno calculadas utilizando ambas as metodologias.

Observamos boa concordância entre as energias de reorganização dos buracos determinadas com cada método. Em ambos os casos $\lambda_{i n}^{e}>\lambda_{i n}^{b}$, porém as discrepâncias são significativas nos resultados obtidos a nível DFTB. Tal comportamento aponta novamente para limitações na caracterização de estados aniônicos (ver Seção 3.2.2) que possivelmente possam vir a ser contornadas a partir de alterações no processo de parametrização dos arquivos de Slater-Koster, como por exemplo, aumentar os pesos atribuídos às energias de atomização [70] ou até mesmo incorporar dados experimentais de $E A$ e $I P$ na função de pontuação (2.65).

Limitações computacionais impossibilitaram a otimização de geometria dos íons do doador e do aceitador eletrônico, e consequentemente a determinação das energias intramoleculares de reorganização. 


\section{Capítulo 4}

\section{Conclusões}

As principais vertentes exploradas no presente trabalho dizem respeito a validação da metodologia LC-TD-DFTB em conjunto aos parâmetros de Slater-Koster OB2+S, o levantamento de espectros de absorção de compostos oticamente ativos, com enfoque no aceitador DTP-IC-4Ph, e a determinação de grandezas relacionadas às células solares.

Como ponto de partida, a legitimação da inflexibilidade na escolha das funções de base foi verificada para os compostos $\pi$-conjugados a partir da convergência entre as geometrias e das suaves flutuações nas energias tanto do estado fundamental quanto dos estados excitados. As isosuperfícies e energias dos orbitais HOMO e LUMO assim como os pequenos deslocamentos rígidos observados nos espectros de absorção sustentam essa premissa. A otimização do parâmetro de longo alcance $(\omega)$ se mostra sensível frente a escolha do método de estrutura eletrônica mas sugere que, dado o critério de otimização, o parâmetro é mantido fixo para uma classe de compostos quimicamente semelhantes, nesse contexto os HPAs. Os critérios de otimização utilizados foram fundamentados no Teorema de Janak e salientaram limitações do método LC-TD-DFTB/OB2+S no que concerne a caracterização da energia do LUMO e de sistemas de camada aberta. Com auxílio de valores experimentais durante o processo de otimização, o gap de energia deixa de ser superestimado e passa a concordar tanto com os cálculos a nível DFT/ $/$ B97X-D/6-31G(d,p) quanto com os valores experimentais. Potenciais de ionização, afinidades eletrônicas, geometrias e espectros de absorção também foram beneficiados da alteração no parâmetro ótimo, sugerindo um ganho global.

A incorporação das cadeias laterais no aceitador eletrônico DTP-IC-4Ph se mostrou de fato pouco importante no que diz respeito aos estados excitados. Em especial, o primeiro pico dos espectros de absorção é invariante frente a alteração, mantendo a absorção no vermelho e preservando tanto a intensidade quanto a largura da banda. O restante do espectro visível sofreu pequenas variações, marcadamente, no formato dos picos. A con- 
cordância não se manteve para as intensidades do monômero de PBDB-T, que também acaba sofrendo variações no valor otimizado de $\omega$. Quando consideramos o espectro decorrente de um conjunto de 1000 coordenadas nucleares por meio da abordagem NEA atrelada ao método LC-TDDFTB/OB2+S, a ausência de efeitos do ambiente se destaca como um dos fatores limitantes para a reprodução dos dados experimentais, porém ainda assim descreve qualitativamente a absorção na região do visível para o composto aceitador. No caso do monômero do doador PBDB-T a ausência dos demais oligômeros compromete as energias dos orbitais e consequentemente a descrição do espectro como um todo, motivando estudos já em andamento que levam em consideração dois ou mais oligômeros. O red shift observado nos espectros dos HPAs na presença de solvente já seria uma primeira correção importante para a concordância com os resultados experimentais, e quando levamos em conta a presença de átomos de oxigênio, nitrôgenio e enxofre na composição dos doadores e aceitadores é de se esperar maiores alterações.

A determinação das constantes dielétricas a partir do modelo de ClausiusMossotti fornece bons resultados quando utilizamos o método DFT mas sofre das polarizabilidades subestimadas do DFTB. Ainda que formalmente seria apropriado considerar a densidade eletrônica na determinação do volume em ambos os formalismos, a utilização de raios de van der Waals resultou em volumes ligeiramente menores que os obtidos via $D F T$, o que acaba por compensar parte das discrepâncias na polarizabilidade mas ainda assim compromete a qualidade dos resultados. As estimativas a partir do ajuste que utiliza de dados experimentais são, a priori, mais adequadas. As altas constantes dielétricas dos compostos ativos atreladas ao momento de dipolo permanente não nulo tendem a aumentar a polarização na presença de solventes, ressaltando a importância dos efeitos do ambiente.

Energias de ligação dos éxcitons e energias intramoleculares de reorganização completam as propriedades exploradas. A primeira delas é pouco sensível frente a escolha do parâmetro de longo alcance e resulta em quasipartículas mais fortemente ligadas nos cálculos via DFTB dos HPAs. Para os demais compostos, ainda que as metodologias não sejam confrontadas, as menores energias são consistentes com a utilização das moléculas no contexto de células solares e trazem o resultado inusitado de que a adição das cadeias laterais acaba por diminuir $E_{b}$, favorecendo a dinâmica de conversão de energia solar em elétrica. As energias intramoleculares 
de reorganização convergem apenas para os portadores de carga positivos, reforçando a limitação na descrição de sistemas de cada aberta mas identificando que tais restrições se concentram na caracterização de sistemas aniônicos.

Uma vez que o conjunto OB2 de parâmetros de Slater-Koster com adição do átomo de enxofre $(\mathrm{OB} 2+\mathrm{S})$ ainda não está disponível publicamente, suas limitações são em parte desconhecidas, o que atribui um caráter pioneiro ao projeto que traz resultados até então não explorados. A validação da metodologia e os cálculos das grandezas físicas de interesse contribuem para a expansão da literatura relacionada ao formalismo, e o levantamento dos espectros de absorção dos compostos DTP-IC-4Ph e PBDB-T, recorrendo apenas a mecânica quântica, foram possibilitados devido ao ganho computacional atrelado à DFTB.

Além da incorporação de efeitos do ambiente, os próximos passos em direção a modelagem de células solares envolvem o cálculo do acoplamento eletrônico $(V)$ entre estados excitados e estados de transferência de carga (CT), que é usualmente determinado a partir da interação de dímeros $[80,56]$ e configura o ponto central, juntamente as energias intramoleculares reorganização, para a determinação das mobilidades dos portadores de carga $\left(k_{i}\right)$. Energias essas, que podem ser melhor exploradas considerando a contribuição externa do solvente, usualmente estimada a partir do modelo de duas esferas [114]. Por fim, refinamentos no modelo semiclássico de transporte e o estudo da dinâmica de estados excitados configuram outros temas relevantes para a estimativa efetiva da eficiência de conversão dos dispositivos fotovoltaicos orgânicos. 


\section{Referências}

[1] U.S. Energy Information Administration. International Energy Outlook 2019. Technical report, U.S. Departament of Energy, 2019. 20

[2] Xiang Xu, Jingyang Xiao, Guichuan Zhang, Long Wei, Xuechen Jiao, Hin Lap Yip, and Yong Cao. Interface-enhanced organic solar cells with extrapolated T80 lifetimes of over 20 years. 65:208-216, 2020. 20

[3] Angélique Léonard Saïcha Gerbinet, Sandra Belboom. Life cycle analysis (LCA) of photovoltaic panels: A review. Renewable and Sustainable Energy Reviews, 38:747-753, 2014. 20

[4] Sebastien Lizin, Steven Van Passel, Ellen De Schepper, Wouter Maes, Laurence Lutsen, and Jean Manca Dirk Vanderzande. Life cycle analyses of organic photovoltaics: a review. Energy Environment Science, 6:3136-3149, 2013. 20

[5] Frederik C. Krebs, Nieves Espinosa, Markus Hösel, Roar R. Søndergaard, and Mikkel Jørgensen. 25th Anniversary Article: Rise to Power - OPV-Based Solar Parks. Adv. Mater., 26, 2014. 29-39. 20

[6] NREL (National Renewable Energy Laboratory). Best Research-Cell Efficiencies. https://www.nrel.gov/pv/cell-efficiency.html, 2021. 8, 20, 21

[7] NREL (National Renewable Energy Laboratory). Organic photovoltaic solar cells. https://www.nrel.gov/pv/ organic-photovoltaic-solar-cells.html. 8, 22

[8] M.C. Scharber and N.S. Sariciftci. Efficiency of bulk-heterojunction organic solar cells. Progress in Polymer Science, 38:1932, 2013. 8, 22

[9] Pei Cheng, Gang Li, Xiaowei Zhan, and Yang Yang. Next-generation organic photovoltaics based on non-fullerene acceptors. Nature Photonics, 12:131-142, 2018. 8, 23, 24, 27

[10] Cenqi Yan, Stephen Barlow, Zhaohui Wang, He Yan, Alex K.Y. Jen, Seth R. Marder, and Xiaowei Zhan. Non-fullerene acceptors for organic solar cells. Nature Reviews Materials, 3:1-19, 2018. 23, 25 
[11] Nicole Bauer, Qianqian Zhang, Jingbo Zhao, Long Ye, Joo Hyun Kim, Iordania Constantinou, Liang Yan, Franky So, Harald Ade, He Yan, and Wei You. Comparing non-fullerene acceptors with fullerene in polymer solar cells: a case study with FTAZ and PyCNbTAZ. Journal of Materials Chemistry A, 5:4886-4893, 2017. 23

[12] Riccardo Po, Andrea Bernardi, Anna Calabrese, Chiara Carbonera, Gianni Corso, and Andrea Pellegrino. From lab to fab: How must the polymer solar cell materials design change?-an industrial perspective. Energy and Environmental Science, 7:925-943, 2014. 23

[13] Hua Sun, Fei Chen, and Zhi Kuan Chen. Recent progress on nonfullerene acceptors for organic photovoltaics. Materials Today, pages 94-118, 2019. 25

[14] Yuze Lin, Jiayu Wang, Zhi Guo Zhang, Huitao Bai, Yongfang Li, Daoben Zhu, and Xiaowei Zhan. An electron acceptor challenging fullerenes for efficient polymer solar cells. Advanced Materials, 27:11701174, 2015. 25

[15] Yang Wang, Bin Liu, Chang Woo Koh, Xin Zhou, Huiliang Sun, Jianwei Yu, Kun Yang, Hang Wang, Qiaogan Liao, Han Young Woo, and Xugang Guo. Facile Synthesis of Polycyclic Aromatic Hydrocarbon (PAH)-Based Acceptors with Fine-Tuned Optoelectronic Properties: Toward Efficient Additive-Free Nonfullerene Organic Solar Cells. Advanced Energy Materials, 9(24):1803976, 2019. 8, 10, 25, 26, 27, 71, 86, 95, 106, 112, 122

[16] L. Ye, X. Jiao, M. Zhou, S. Zhang, H. Yao, W. Zhao, A. Xia, H. Ade, and J. Hou. Manipulating Aggregation and Molecular Orientation in All-Polymer Photovoltaic Cells. Advanced Materials, 27:6046-6054, 2015. 25, 28

[17] Bin Kan, Huanran Feng, Xiangjian Wan, Feng Liu, Xin Ke, Yanbo Wang, Yunchuang Wang, Hongtao Zhang, Chenxi Li, Jianhui Hou, and Yongsheng Chen. Small-Molecule Acceptor Based on the Heptacyclic Benzodi(cyclopentadithiophene) Unit for Highly Efficient Nonfullerene Organic Solar Cells. Journal of the American Chemical Society, 139:4929-4934, 2017. 25

[18] Xianhe Zhang, Yumin Tang, Peng Yang, Kun Chen, and Xugang Guo. Additive-Free Non-Fullerene Organic Solar Cells, 2019. 27 
[19] Veaceslav Coropceanu, Xian-Kai Chen, Tonghui Wang, Zilong Zheng, and Jean-Luc Brédas. Charge-transfer electronic states in organic solar cells. Nature Reviews Materials, 4:689-707, 2019. 27, 122

[20] L. Benatto, C. A.M. Moraes, De Jesus, M. Bassi, L. Wouk, L. S. Roman, and M. Koehler. Kinetic Modeling of the Electric Field Dependent Exciton Quenching at the Donor-Acceptor Interface. Journal of Physical Chemistry C, 125:4436-4448, 2021. 27

[21] D. Fazzi, M. Barbatti, and W. Thiel. Hot and Cold Charge-Transfer Mechanisms in Organic Photovoltaics: Insights into the Excited States of Donor/Acceptor Interfaces. The Journal of Physical Chemistry Letters, 8:4727, 2017. 27, 44, 77

[22] L. Benatto, C. F.N. Marchiori, M. G.E. Da Luz, and M. Koehler. Electronic and structural properties of fluorene-thiophene copolymers as function of the composition ratio between the moieties: A theoretical study. Physical Chemistry Chemical Physics, 20:20447-20458, 2018. $27,44,77$

[23] T N Márcio Varella, Ljiljana Stojanovic, Van Quan Vuong, Stephan Irle, Thomas A Niehaus, and Mario Barbatti. How the Size and Density of Charge-Transfer Excitons Depend on Heterojunction's Architecture. The Journal of Physical Chemistry C, 125:5458-5474, 2021. $27,29,87,172$

[24] Sunsun Li, Long Ye, Wenchao Zhao, Shaoqing Zhang, Subhrangsu Mukherjee, Harald Ade, and Jianhui Hou. Energy-level Modulation of Small-Molecule Electron Acceptors to Achieve over 12\% Efficiency in Polymer Solar Cells. Advanced Materials, 28:9423-9429, 2016. 11, 28, 111

[25] Wenchao Zhao, Deping Qian, Shaoqing Zhang, Sunsun Li, Olle Inganäs, Feng Gao, and Jianhui Hou. Fullerene-Free Polymer Solar Cells with over 11\% Efficiency and Excellent Thermal Stability. Advanced Materials, 28:4734-4739, 2016. 28, 98

[26] Xi Liu, Boming Xie, Chunhui Duan, Zhaojing Wang, Baobing Fan, Kai Zhang, Baojun Lin, Fallon J.M. Colberts, Wei Ma, René A.J. Janssen, Fei Huang, and Yong Cao. A high dielectric constant nonfullerene acceptor for efficient bulk-heterojunction organic solar cells. 2018. 28,74 
[27] Zhong Zheng, Huifeng Yao, Long Ye, Ye Xu, Shaoqing Zhang, and Jianhui Hou. PBDB-T and its derivatives: A family of polymer donors enables over $17 \%$ efficiency in organic photovoltaics. Materials Today, 35:115-130, 2020. 8, 28

[28] Hao Zhang, Huifeng Yao, Junxian Hou, Jie Zhu, Jianqi Zhang, Wanning Li, Runnan Yu, Bowei Gao, Shaoqing Zhang, and Jianhui Hou. Over 14\% Efficiency in Organic Solar Cells Enabled by Chlorinated Nonfullerene Small-Molecule Acceptors. Advanced Materials, 30:1-7, 2018. 28

[29] Sunsun Li, Long Ye, Wenchao Zhao, Hongping Yan, Bei Yang, Delong Liu, Wanning Li, Harald Ade, and Jianhui Hou. A Wide Band Gap Polymer with a Deep Highest Occupied Molecular Orbital Level Enables 14.2\% Efficiency in Polymer Solar Cells. Journal of the American Chemical Society, 140:7159-7167, 2018. 28

[30] José David M. Vianna, Adalberto Fazzio, and Sylvio Canuto. Relação de Thomas-Fermi. In Teoria Quântica de Moléculas e Sólidos: Simulação Computacional, pages 125, 214-218. Livraria da Física, 2004. 29, 32, 42

[31] C. Fiolhais, F. Nogueira, and M. Marques. Density Functionals for Non-relativistic Coulomb Systems in the New Century, pages 3, 2024, 25-32, 51. Springer-Verlag Berlin Heidelberg, 2003. 29, 41, 95

[32] R. Parr and W. Yang. Density-Functional Theory of Atoms and Molecules, pages 163-168. Oxford University Press, 1989. 29, 86

[33] Wolfram Koch and Max C. Holthausen. A Chemist's Guide to Density Functional Theory. Wiley-VCH, second edition, 2001. 29

[34] Carsten A. Ullrich. Time-Dependent Density-Functional Theory: Concepts and Applications. Oxford University Press, 2012. 8, 29, 42,47

[35] V. Lutsker, B. Aradi, and T. A. Niehaus. Implementation and benchmark of a long-range corrected functional in the density functional based tight-binding method. Journal of Chemical Physics, 143:184107, 2015. 29, 64, 65, 68, 69, 96, 99

[36] Julian J. Kranz, Marcus Elstner, Bálint Aradi, Thomas Frauenheim, Vitalij Lutsker, Adriel Dominguez Garcia, and Thomas A. Niehaus. 
Time-Dependent Extension of the Long-Range Corrected Density Functional Based Tight-Binding Method. Journal of Chemical Theory and Computation, 13:1737-1747, 2017. 29, 64, 66, 67, 93

[37] Beatrix M. Bold, Monja Sokolov, Sayan Maity, Marius Wanko, Philipp M. Dohmen, Julian J. Kranz, Ulrich Kleinekathöfer, Sebastian Höfener, and Marcus Elstner. Benchmark and performance of longrange corrected time-dependent density functional tight binding (LCTD-DFTB) on rhodopsins and light-harvesting complexes. Physical Chemistry Chemical Physics, 22:10500-10518, 2020. 29, 99

[38] Ala Aldin M. H. M. Darghouth, Mark E. Casida, Xi () Zhu, Bhaarathi Natarajan, Haibin () Su, Alexander Humeniuk, Evgenii Titov, Xincheng () Miao, and Roland Mitrić. Effect of Varying the TD-lcDFTB Range-Separation Parameter on Charge and Energy Transfer in a Model Pentacene/Buckminsterfullerene Heterojunction. The Journal of Chemical Physics, 154(5):054102, 2021. 29

[39] Sharma S.R.K.C. Yamijala and Pengfei Huo. Direct Nonadiabatic Simulations of the Photoinduced Charge Transfer Dynamics. Journal of Physical Chemistry A, 125:628-635, 2021. 29

[40] Thomas Körzdörfer, John S. Sears, Christopher Sutton, and Jean Luc Brédas. Long-range corrected hybrid functionals for -conjugated systems: Dependence of the range-separation parameter on conjugation length. Journal of Chemical Physics, 135:204107, 2011. 30, 98

[41] Ross Larsen. Simple Extrapolation Method to Predict the Electronic Structure of Conjugated Polymers from Calculations on Oligomers. Journal of Physical Chemistry C, 120:9650-9660, 2016. 30, 98, 111

[42] Sanjio S. Zade and Michael Bendikov. From oligomers to polymer: Convergence in the HOMO-LUMO gaps of conjugated oligomers. Organic Letters, 8:5243-5246, 2006. 30

[43] Max Born and Kun Huang. Dynamical Theory of Crystal Lattices. Oxford University Press, 1954. 32

[44] J. Rachid Mohallem and F. Vasconcellos Prudente. Métodos além da Aproximação Born-Oppenheimer. Editora Livraria da Física, 2007. 33,143 
[45] Michael Baer. Introduction to the theory of electronic non-adiabatic coupling terms in molecular systems. Physics Reports, 358:79-81, 2002. 33

[46] Thirty years of density functional theory in computational chemistry: An overview and extensive assessment of 200 density functionals. Molecular Physics, 115:2315-2372, 2017. 34, 41

[47] Reiner M. Dreizler and Eberhard K. U. Gross. Density Functional Theory of Relativistic Systems. Springer Berlin Heidelberg, 1990. 37

[48] John P. Perdew and Karla Schmidt. Jacob's ladder of density functional approximations for the exchange-correlation energy. AIP Conference Proceedings, 577(1):1-20, 2001. 41

[49] J. Perdew and A. Zunder. Self-interaction correction to densityfunctional approximations for many-electron systems. Physical Review $B, 23: 5048-5079,1981.42$

[50] Jeng Da Chai and Martin Head-Gordon. Systematic optimization of long-range corrected hybrid density functionals. Journal of Chemical Physics, 128(8):084106, 2008. 42, 148, 149

[51] Jeng Da Chai and Martin Head-Gordon. Long-range corrected hybrid density functionals with damped atom-atom dispersion corrections. Physical Chemistry Chemical Physics, 10:6615-6620, 2008. 42, 149

[52] Axel D. Becke. Density-functional thermochemistry. V. Systematic optimization of exchange-correlation functionals. Journal of Chemical Physics, 107:8554-8560, 1997. 43, 85, 148

[53] Stefan Grimme. Semiempirical GGA-Type Density Functional Constructed with a Long-Range Dispersion Correction. Journal of Computational Chemistry, 27:1787-1799, 2006. 44

[54] Saju Joseph, Mahesh Kumar Ravva, and Jean Luc Bredas. ChargeTransfer Dynamics in the Lowest Excited State of a PentaceneFullerene Complex: Implications for Organic Solar Cells. Journal of Physical Chemistry Letters, 8:5171-5176, 2017. 44, 77

[55] Z. Zheng, N. R. Tummala, Y.-T. Fu, V. Coropceanu, and J.-L. Brédas. Charge-Transfer States in Organic Solar Cells: Understanding the Impact of Polarization, Delocalization, and Disorder. ACS Applied Materials Interfaces, 9:18095-18102, 2017. 44, 77 
[56] Leandro Benatto and Marlus Koehler. Effects of Fluorination on Exciton Binding Energy and Charge Transport of $\pi$-Conjugated Donor Polymers and the ITIC Molecular Acceptor: A Theoretical Study. Journal of Physical Chemistry C, 123:6395-6406, 2019. 44, 77, 126

[57] De Almeida Sousa, Karlisson Rodrigo, Leandro Benatto, Luana Wouk, Lucimara Stolz Roman, and Marlus Koehler. Effects of nonhalogenated solvent on the main properties of a solution-processed polymeric thin film for photovoltaic applications: A computational study. Physical Chemistry Chemical Physics, 22:9693-9702, 2020. 44, 77

[58] T. Tsuneda and K. Hirao. Long-range correction for density functional theory. WIREs: Computational Molecular Science, 4:375, 2014. 44, 77, 91, 96

[59] R. Ditchfield, W.J Hehre, and J. A. Pople. Self-Consistent Molecular-Orbital Methods. IX. An Extended Gaussian-Type Basis for Molecular-Orbital Studies of Organic Molecules. The Journal of Chemical Physics, 54:724-728, 1971. 44

[60] A. Szabo and N. S. Ostlund. Modern Quantum Chemistry: Introduction to Advanced Electronic Structure Theory. McGraw-Hill Publishing Company, 1996. 44

[61] J. Rajagopal, A. K. Callaway. Inhomogeneous Electron Gas. Physical Review B, 7:1912-1919, 1973. 46, 47

[62] Mark E. Casida. Time-Dependent Density Functional Response Theory for Molecules, pages 155-192. World Scientific. 46

[63] Takao Tsuneda. Density Functional Theory in Quantum Chemistry, pages $91,125-130$. Springer, 2014. 47, 85

[64] Zeng Hui Yang and Kieron Burke. Nonexistence of a Taylor expansion in time due to cusps. Physical Review A - Atomic, Molecular, and Optical Physics, 88:1-14, 2013. 47, 48

[65] Robert Van Leeuwen. Causality and symmetry in time-dependent density-functional theory. Physical Review Letters, 80:1280-1283, 1998. 47 
[66] Robert Van Leeuwen. Mapping from Densities to Potentials in Time-Dependent Density-Functional Theory. Physical Review Letters, 82:3863-3866, 1999. 47, 48

[67] Robert Van Leeuwen. Key concepts in time-dependent densityfunctional theory, volume 15. 47

[68] M. Ruggenthaler and R. van Leeuwen. Global fixed-point proof of time-dependent density-functional theory. Europhysics Letters Association, 95:13001, 2011. 48

[69] M. Ruggenthaler, K. J.H. Giesbertz, M. Penz, and R. Van Leeuwen. Density-potential mappings in quantum dynamics. Physical Review A - Atomic, Molecular, and Optical Physics, 85:052504, 2012. 48

[70] Van Quan Vuong, Jissy Akkarapattiakal Kuriappan, Maximilian Kubillus, Julian J. Kranz, Thilo Mast, Thomas A. Niehaus, Stephan Irle, and Marcus Elstner. Parametrization and Benchmark of LongRange Corrected DFTB2 for Organic Molecules. Journal of Chemical Theory and Computation, 14:115-125, 2018. 57, 62, 64, 87, 123

[71] Augusto F. Oliveira, Gotthard Seifert, Thomas Heine, and Hélio A. Duarte. Density-functional based tight-binding: An approximate DFT method. Journal of the Brazilian Chemical Society, 20:11931205, 2009. 57

[72] D. Porezag, Th Frauenheim, Th Köhler, G. Seifert, and R. Kaschner. Construction of tight-binding-like potentials on the basis of density-functional theory: Application to carbon. Physical Review $B, 51: 12947-12957,1995.57,62$

[73] Roi Baer and Daniel Neuhauser. Density Functional Theory with Correct Long-Range Asymptotic Behavior. Physical Review Letters, $94: 2-5,2005$. 64, 85

[74] Ester Livshits and Roi Baer. A well-tempered density functional theory of electrons in molecules. Physical Chemistry Chemical Physics, 9:2932-2941, 2007. 64, 85

[75] John P. Perdew, Kieron Burke, and Matthias Ernzerhof. Generalized gradient approximation made simple. Physical Review Letters, $77: 3865-3868,1996.65$ 
[76] Michael Seth and Tom Ziegler. Range-separated exchange functionals with slater-type functions. Journal of Chemical Theory and Computation, 8:901-907, 2012. 65

[77] Pekka Koskinen and Ville Mäkinen. Density-functional tight-binding for beginners. Computational Materials Science, 47:237-253, 2009. 69

[78] A. Domínguez, B. Aradi, T. Frauenheim, V. Lutsker, and T. A. Niehaus. Extensions of the time-dependent density functional based tight-binding approach. Journal of Chemical Theory and Computation, 9:4901-4914, 2013. 69

[79] J. Tomasi, B. Mennucci, and R. Cammi. Quantum mechanical continuum solvation models. Chemical Reviews, 105:2999-3093, 2005. 71, 77

[80] Edward F. Valeev, Veaceslav Coropceanu, Demetrio A. Da Silva Filho, Seyhan Salman, and Jean Luc Brédas. Effect of electronic polarization on charge-transport parameters in molecular organic semiconductors. Journal of the American Chemical Society, 128:9882-9886, 2006. $74,122,126$

[81] J. Patrick Zobel, Juan J. Nogueira, and Leticia González. Finitetemperature Wigner phase-space sampling and temperature effects on the excited-state dynamics of 2-nitronaphthalene. Physical Chemistry Chemical Physics, 21:13906-13915, 2019. 75

[82] Fábris Kossoski and Mario Barbatti. Nuclear Ensemble Approach with Importance Sampling. Journal of Chemical Theory and Computation, 14:3173-3183, 2018. 76, 108

[83] Junwei Lucas Bao, Laura Gagliardi, and Donald G. Truhlar. SelfInteraction Error in Density Functional Theory: An Appraisal. Journal of Physical Chemistry Letters, 9:2353-2358, 2018. 77, 91

[84] Tamar Stein, Leeor Kronik, and Roi Baer. Prediction of chargetransfer excitations in coumarin-based dyes using a range-separated functional tuned from first principles. The Journal of Chemical Physics, 131(24):244119, 2009. 77, 85, 91

[85] Tamar Stein, Helen Eisenberg, Leeor Kronik, and Roi Baer. Fundamental gaps in finite systems from eigenvalues of a generalized kohn-sham method. Physical Review Letters, 105:266802:1-4. 77, 85 
[86] Chemcraft - graphical software for visualization of quantum chemistry computations. https://www. chemcraftprog.com/help/ structcomparerwindow.html. 78

[87] J. F. Janak. Proof that $\frac{\partial e}{\partial n_{i}}=\epsilon$ in density-functional theory. Physical Review B, 18:7165-7168, 1978. 85

[88] Gaussian 09, Revision A.02, M. J. Frisch, G. W. Trucks, H. B. Schlegel, G. E. Scuseria, M. A. Robb, J. R. Cheeseman, G. Scalmani, V. Barone, G. A. Petersson, H. Nakatsuji, X. Li, M. Caricato, A. Marenich, J. Bloino, B. G. Janesko, R. Gomperts, B. Mennucci, H. P. Hratchian, J. V. Ortiz, A. F. Izmaylov, J. L. Sonnenberg, D. Williams-Young, F. Ding, F. Lipparini, F. Egidi, J. Goings, B. Peng, A. Petrone, T. Henderson, D. Ranasinghe, V. G. Zakrzewski, J. Gao, N. Rega, G. Zheng, W. Liang, M. Hada, M. Ehara, K. Toyota, R. Fukuda, J. Hasegawa, M. Ishida, T. Nakajima, Y. Honda, O. Kitao, H. Nakai, T. Vreven, K. Throssell, J. A. Montgomery, Jr., J. E. Peralta, F. Ogliaro, M. Bearpark, J. J. Heyd, E. Brothers, K. N. Kudin, V. N. Staroverov, T. Keith, R. Kobayashi, J. Normand, K. Raghavachari, A. Rendell, J. C. Burant, S. S. Iyengar, J. Tomasi, M. Cossi, J. M. Millam, M. Klene, C. Adamo, R. Cammi, J. W. Ochterski, R. L. Martin, K. Morokuma, O. Farkas, J. B. Foresman, and D. J. Fox, Gaussian, Inc., Wallingford CT, 2016. 11, 86, 112, 114

[89] B. Hourahine, B. Aradi, V. Blum, F. Bonafé, A. Buccheri, C. Camacho, C. Cevallos, M. Y. Deshaye, T. Dumitrică, A. Dominguez, S. Ehlert, M. Elstner, T. van der Heide, J. Hermann, S. Irle, J. J. Kranz, C. Köhler, T. Kowalczyk, T. Kubař, I. S. Lee, V. Lutsker, R. J. Maurer, S. K. Min, I. Mitchell, C. Negre, T. A. Niehaus, A. M. N. Niklasson, A. J. Page, A. Pecchia, G. Penazzi, M. P. Persson, J. Řezáč, C. G. Sánchez, M. Sternberg, M. Stöhr, F. Stuckenberg, A. Tkatchenko, V. W.-z. Yu, and T. Frauenheim. DFTB+, a software package for efficient approximate density functional theory based atomistic simulations. The Journal of Chemical Physics, 152(12):124101, 2020. 86

[90] Martin C. R. Cockett, Hiroyuki Ozeki, Katsuhiko Okuyama, and Katsumi Kimura. Vibronic coupling in the ground cationic state of naphthalene: A laser threshold photoelectron [zero kinetic energy (zeke)-photoelectron] spectroscopic study. The Journal of Chemical Physics, 98:7763, 1993. 90 
[91] J. Schiedt, W.J. Knott, K. Le Barbu, E.W. Schlag, and R. Weinkauf. Microsolvation of similar-sized aromatic molecules: Photoelectron spectroscopy of bithiophene-, azulene-, and naphthalene-water anion clusters. The Journal of Chemical Physics, 113:9470-9478, 2000. 90

[92] J.W. Hager and S.C. Wallace. Two-laser photoionization supersonic jet mass spectrometry of aromatic molecules. Analytical Chemistry, 60:5-10, 1988. 90

[93] N. Ando, S. Kokubo, M. Mitsui, and A. Nakajima. Photoelectron spectroscopy of pyrene cluster anions, (pyrene)(-)(n) $(\mathrm{n}=1-20)$. Chemical Physics Letters, 389:279-283, 2004. 90

[94] Oleg Vydrov. Correcting the Self-Interaction Error of Approximate Density Functionals. PhD thesis, Rice University, 2007. 91

[95] E. Bundgaard, F. Krebs, and Mikkel Jørgensen. Notas de aula do curso Organic Solar Cells - Theory and Practice, 2019. 93

[96] E. J. Baerends, O. V. Gritsenko, and R. Van Meer. The Kohn-Sham gap, the fundamental gap and the optical gap: The physical meaning of occupied and virtual Kohn-Sham orbital energies. Physical Chemistry Chemical Physics, 15:16408-16425, 2013. 96

[97] Gang Zhang and Charles B. Musgrave. Comparison of DFT methods for molecular orbital eigenvalue calculations. Journal of Physical Chemistry A, 111:1554-1561, 2007. 96

[98] Takao Tsuneda, Jong Won Song, Satoshi Suzuki, and Kimihiko Hirao. On koopmans' theorem in density functional theory. Journal of Chemical Physics, 133:174101, 2010. 96

[99] T.A. Niehaus, M. Elstner, Th. Frauenheim, and S. Suhaia. Application of an approximate density-functional method to sulfur containing compounds. Journal of Molecular Structure: THEOCHEM, 541:185-194, 2001. 96

[100] Christopher J. Cramer. Essentials of Computational Chemistry: Theories and Models. John Wiley \& Sons, 2nd edition, 2004. 96

[101] Daniele Selli, Gianluca Fazio, and Cristiana Di Valentin. Modelling Realistic TiO2 nanospheres: A benchmark study of SCC-DFTB 
against hybrid DFT. The Journal of Chemical Physics, 147:164701, 2017. 98

[102] Fabio Trani, Giovanni Scalmani, Guishan Zheng, Ivan Carnimeo, Michael J. Frisch, and Vincenzo Barone. Time-dependent density functional tight binding: New formulation and benchmark of excited states. Journal of Chemical Theory and Computation, 7:3304-3313, 2011. 99

[103] Mario Barbatti, Matthias Ruckenbauer, Felix Plasser, Jiri Pittner, Giovanni Granucci, Maurizio Persico, and Hans Lischka. Newton$\mathrm{X}$ : a surface-hopping program for nonadiabatic molecular dynamics. WIREs: Computational Molecular Science, 4:26-33, 2014. 106

[104] Sanwu Wang. Generalization of the Thomas-Reiche-Kuhn and the Bethe sum rules. Physical Review A, 60:262-266, 1999. 107

[105] Shuming Bai, Ritam Mansour, Ljiljana Stojanović, Josene M. Toldo, and Mario Barbatti. On the origin of the shift between vertical excitation and band maximum in molecular photoabsorption. Journal of Molecular Modelling, 26, 2020. 108

[106] Christian Reichardt and Thomas Welton. Solvents and Solvent Effects in Organic Chemistry. Wiley-VCH, 4th edition, 2010. 115

[107] Ove Christiansen, Christof Hättig, and Poul Jørgensen. Ground and excited state polarizabilities and dipole transition properties of benzene from coupled cluster response theory. 55:509-524, 1999. 115

[108] N. Ghoneim and P. Suppan. Solvatochromic shifts of non-dipolar molecules in polar solvents. 51:1043-1050, 1995. 115

[109] Pabitra K. Nayak and N. Periasamy. Calculation of electron affinity, ionization potential, transport gap, optical band gap and exciton binding energy of organic solids using 'solvation' model and DFT. Organic Electronics, 10:1396-1400, 2009. 117

[110] Stefan Grimme, Jens Antony, Stephan Ehrlich, and Helge Krieg. A consistent and accurate ab initio parametrization of density functional dispersion correction (DFT-D) for the 94 elements $\mathrm{H}-\mathrm{Pu}$. The Journal of Chemical Physics, 132, 2010. 117, 181 
[111] Ishii Kikujiro, Kinoshita Minoru, and Kuroda Haruo. Dielectric Constant Measurement on Organic Crystalline Powder. Bulletin of the Chemical Society of Japan, 46, 1973. 119

[112] F.M. Dekking, C. Kraaikamp, and L.E. Lopuhaä, H.P. Meester. A Modern Introduction to Probability and Statistics: Understanding Why and How. Spring-Verlag, 2005. 119

[113] Lingyun Zhu, Yuanping Yi, and Zhixiang Wei. Exciton Binding Energies of Nonfullerene Small Molecule Acceptors: Implication for Exciton Dissociation Driving Forces in Organic Solar Cells. Journal of Physical Chemistry C, 122:22309-22316, 2018. 120

[114] Can Leng, Haimei Qin, Yubing Si, and Yi Zhao. Theoretical prediction of the rate constants for exciton dissociation and charge recombination to a triplet state in pcpdtbt with different fullerene derivatives. Journal of Physical Chemistry C, 118:1843-1855, 2014. 122, 126

[115] John P. Perdew and Yue Wang. Accurate and simple analytic representation of the electron-gas correlation energy. Physical Review B, 98:244-249, 1992. 148

[116] Peter Elliott, Sharma Goldson, Chris Canahui, and Neepa T. Maitra. Perspectives on double-excitations in TDDFT. Chemical Physics, 391:110-119, 2011. 160

[117] A. Görling. Density-functional theory for excited states. Physical Review A, 54:3912-3915, 1996. 164

[118] John C. Slater and John G. Kirkwood. The van der Waals forces in gases. Physical Review, 37:682-697, 1931. 171

[119] Thomas A. Halgren. Representation of van der Waals (vdW) Interactions in Molecular Mechanics Force Fields: Potential Form, Combination Rules, and vdW Parameters. Journal of the American Chemical Society, 114:7827-7843. 171

[120] Marcus Elstner, Pavel Hobza, Thomas Frauenheim, Sándor Suhai, and Efthimios Kaxiras. Hydrogen bonding and stacking interactions of nucleic acid base pairs: A density functional-theory based treatment. Journal of Chemical Physics, 114:5149-5155, 2001. 172

[121] Mark A. Heald and Jerry B. Marion. Classical Electromagnetic Radiation. Saunders College Publishing, 3rd edition, 1995. 178 
[122] Alan Corney. Atomic and Laser Spectroscopy. Oxford Classic Series, 2006. 178

[123] Malvin H. Kalos and Paula A. Whitlock. Monte Carlo Methods. WILEY-VCH, 2nd edition, 2008. 181

[124] Gaussian09 manual - Volume. http://wild.life.nctu.edu.tw/ jsyu/compchem/g09/g09ur/k_volume.htm. 181 


\section{Apêndice A}

\section{Expansão de Born-Huang}

Substituindo a hamiltoniana molecular (2.2) e a expansão de BornHuang (2.4) na equação de Schrödinger independente do tempo (2.1), segue que,

$$
\left(\hat{\mathcal{H}}_{n}+\hat{\mathcal{H}}_{e}\right) \sum_{k} \chi_{k}(\boldsymbol{R}) \psi_{k}(\boldsymbol{r} \mid \boldsymbol{R})=E \sum_{k} \chi_{k}(\boldsymbol{R}) \psi_{k}(\boldsymbol{r} \mid \boldsymbol{R})
$$

Tendo em vista que $\left\{\psi_{k}(\boldsymbol{r} \mid \boldsymbol{R})\right\}$ são autoestados de $\hat{\mathcal{H}}_{e}$, é conveniente abrir os termos nucleares, de tal forma que,

$$
(\underbrace{-\sum_{\alpha=1}^{N_{n}} \frac{\nabla_{\alpha}^{2}}{2 m_{\alpha}}}_{T_{n}}+\underbrace{\frac{1}{2} \sum_{\alpha \neq \beta}^{N_{n}} \frac{Z_{\alpha} Z_{\beta}}{\left\|\boldsymbol{R}_{\alpha}-\boldsymbol{R}_{\beta}\right\|}}_{V_{n n}}+\hat{\mathcal{H}}_{e}-E) \sum_{k} \chi_{k}(\boldsymbol{R}) \psi_{k}(\boldsymbol{r} \mid \boldsymbol{R})=0 .
$$

Uma vez que os núcleos estão fixos, o termo puramente nuclear $\left(V_{n n}\right)$ é constante, alterando apenas a escala do problema. A função de onda nuclear se transforma como constante frente a hamiltoniana eletrônica e como vetor frente ao termo cinético nuclear, enquanto a função de onda eletrônica se transforma como vetor frente a hamiltoniana eletrônica e como campo escalar frente ao termo cinético, uma vez que sua dependência em $\boldsymbol{R}$ é paramétrica. Desta forma, utilizando da identidade vetorial,

$$
\nabla^{2}(\phi \boldsymbol{A})=\boldsymbol{A} \nabla^{2} \phi+2(\nabla \phi \cdot \nabla) \boldsymbol{A}+\phi \nabla^{2} \boldsymbol{A}
$$

onde $\phi$ é um campo escalar e $\boldsymbol{A}$ um campo vetorial, podemos reescrever (A.1) como, 


$$
\begin{gathered}
-\sum_{\alpha=1}^{N_{n}} \frac{1}{2 m_{\alpha}} \sum_{k}\left[\chi_{k} \nabla_{\alpha}^{2} \psi_{k}+2\left(\nabla_{\alpha} \psi_{k} \cdot \nabla_{\alpha}\right) \chi_{k}+\psi_{k} \nabla_{\alpha}^{2} \chi_{k}\right]+ \\
\left(\hat{\mathcal{H}}_{e}+\frac{1}{2} \sum_{\alpha \neq \beta}^{N_{n}} \frac{Z_{\alpha} Z_{\beta}}{\left\|\boldsymbol{R}_{\alpha}-\boldsymbol{R}_{\beta}\right\|}-E\right) \sum_{k} \chi_{k}(\boldsymbol{R}) \psi_{k}(\boldsymbol{r} \mid \boldsymbol{R})=0 .
\end{gathered}
$$

Projetando a equação acima sobre um estado eletrônico $\psi_{l}(\boldsymbol{r} \mid \boldsymbol{R})$, que consiste em multiplicar a equação por $\psi_{l}^{*}(\boldsymbol{r} \mid \boldsymbol{R})$, integrando sobre todas as coordenadas eletrônicas, e tendo em vista a relação de ortogonalidade dos autoestados eletrônicos,

$$
\int d \boldsymbol{r} \psi_{n}^{*}(\boldsymbol{r} \mid \boldsymbol{R}) \psi_{m}(\boldsymbol{r} \mid \boldsymbol{R})=\delta_{n m},
$$

onde $\delta_{n m}$ é a delta de Kronecker, obtém-se uma equação para parte nuclear da função de onda,

$$
\begin{array}{r}
-\sum_{\alpha=1}^{N_{n}} \sum_{k}\left(\frac{\left(\nabla_{\alpha}^{2}\right)_{l k}}{2 m_{\alpha}}+\frac{\left(\nabla_{\alpha}^{2}\right)_{l k} \cdot \nabla_{\alpha}}{m_{\alpha}}+\frac{\nabla_{\alpha}^{2}}{2 m_{\alpha}}+\left(\hat{\mathcal{H}}_{e}\right)_{l k} \delta_{l k}\right) \chi_{k}(\boldsymbol{R})+ \\
\left(\frac{1}{2} \sum_{\alpha \neq \beta}^{N_{n}} \frac{Z_{\alpha} Z_{\beta}}{\left\|\boldsymbol{R}_{\alpha}-\boldsymbol{R}_{\beta}\right\|}-E\right) \delta_{l k} \chi_{k}(\boldsymbol{R})=0,
\end{array}
$$

tal que,

$$
\left(\nabla_{\alpha}\right)_{n m} \equiv \int d \boldsymbol{r} \psi_{n}^{*}(\boldsymbol{r} \mid \boldsymbol{R}) \nabla_{\alpha} \psi_{m}(\boldsymbol{r} \mid \boldsymbol{R}) \equiv\left\langle\psi_{n}\left|\nabla_{\alpha}\right| \psi_{m}\right\rangle
$$

$\mathrm{e}$

$$
\left(\nabla_{\alpha}^{2}\right)_{n m} \equiv \int d \boldsymbol{r} \psi_{n}^{*}(\boldsymbol{r} \mid \boldsymbol{R}) \nabla_{\alpha}^{2} \psi_{m}(\boldsymbol{r} \mid \boldsymbol{R}) \equiv\left\langle\psi_{n}\left|\nabla_{\alpha}^{2}\right| \psi_{m}\right\rangle .
$$

Redefinindo a escala de energia pela inclusão do termo de repulsão nuclear,

$$
\mathcal{E}(\boldsymbol{R}) \equiv E-\frac{1}{2} \sum_{\alpha \neq \beta}^{N_{n}} \frac{Z_{\alpha} Z_{\beta}}{\left\|\boldsymbol{R}_{\alpha}-\boldsymbol{R}_{\beta}\right\|},
$$

e separando convenientemente os termos diagonais dos não diagonais, con- 
cluímos que para cada $\alpha$ vale a relação [44],

$$
\left(-\frac{\nabla_{\alpha}^{2}}{2 m_{\alpha}}-\frac{\left(\nabla_{\alpha}^{2}\right)_{l l}}{2 m_{\alpha}}+\left(\hat{\mathcal{H}}_{e}\right)_{l l}-\mathcal{E}(\boldsymbol{R})\right) \chi_{l}(\boldsymbol{R})=\sum_{k \neq l}\left(\frac{\left(\nabla_{\alpha}^{2}\right)_{l k}}{2 m_{\alpha}}+\frac{\left(\nabla_{\alpha}\right)_{l k} \cdot \nabla_{\alpha}}{m_{\alpha}}\right) \chi_{k}(\boldsymbol{R}) .
$$

Note que $\left(\nabla_{\alpha}\right)_{l l}=0$ para funções eletrônicas reais, pois,

$$
0=\nabla_{\alpha} \underbrace{\left(\left\langle\psi_{l} \mid \psi_{l}\right\rangle\right)}_{1}=\left\langle\psi_{l} \nabla_{\alpha} \mid \psi_{l}\right\rangle+\left\langle\psi_{l} \mid \nabla_{\alpha} \psi_{l}\right\rangle=2\left\langle\psi_{l} \mid \nabla_{\alpha} \psi_{l}\right\rangle=2\left(\nabla_{\alpha}\right)_{l l} .
$$




\section{Apêndice B}

\section{Derivação das Equações de Kohn-Sham}

Partindo da equação (2.19) e tendo em vista a expressão (2.15) para a densidade eletrônica assim como as definições (2.16) e (2.17), é possível tomar a variação com respeito aos spin-orbitais $\psi_{i}(\boldsymbol{r})$, ou, convenientemente, com relação ao complexos conjugados,

$$
\frac{\delta}{\delta \psi_{i}^{*}(\boldsymbol{r})}\left\{E[\rho]-\sum_{i, j=1}^{N} \epsilon_{i j}\left(\int d \boldsymbol{r} \psi_{i}^{*}(\boldsymbol{r}) \psi_{j}(\boldsymbol{r})-\delta_{i j}\right)\right\}=0
$$

Para os funcionais que possuem expressões fechadas em termos de $\rho[\boldsymbol{r}]$ é conveniente utilizar da regra da cadeia. Seja $\mathcal{F}$ um funcional da densidade $\rho(\boldsymbol{r})$, vale que,

$$
\frac{\delta \mathcal{F}[\rho]}{\delta \psi_{i}^{*}(\boldsymbol{r})}=\frac{\delta \mathcal{F}[\rho(\boldsymbol{r})]}{\delta \rho(\boldsymbol{r})} \frac{\delta \rho(\boldsymbol{r})}{\delta \psi_{j}^{*}(\boldsymbol{r})} \frac{\delta \psi_{j}^{*}(\boldsymbol{r})}{\delta \psi_{i}^{*}(\boldsymbol{r})} \stackrel{(2.15)}{=} 2 \psi_{i} \frac{\delta \mathcal{F}[\rho]}{\delta \rho(\boldsymbol{r})} .
$$

A seguir são apresentados os cálculos de cada um dos termos.

\section{B.1 Integral de Coulomb}

Considerando uma variação infinitesimal da densidade na expressão (2.6), segue que,

$$
\begin{aligned}
\delta J[\rho] & =J[\rho+\delta \rho]-J[\rho] \\
& =\frac{1}{2} \iint d \boldsymbol{r} d \boldsymbol{r}^{\prime} \frac{[\rho(\boldsymbol{r})+\delta \rho(\boldsymbol{r})]\left[\rho\left(\boldsymbol{r}^{\prime}\right)+\delta \rho\left(\boldsymbol{r}^{\prime}\right)\right]}{\left\|\boldsymbol{r}-\boldsymbol{r}^{\prime}\right\|}-\frac{1}{2} \iint d \boldsymbol{r} d \boldsymbol{r}^{\prime} \frac{\rho(\boldsymbol{r}) \rho\left(\boldsymbol{r}^{\prime}\right)}{\left\|\boldsymbol{r}-\boldsymbol{r}^{\prime}\right\|} .
\end{aligned}
$$

Uma vez que a primeira das cinco integrais anula a última, desconsiderando a integral de segunda ordem (que contém $\delta \rho(\boldsymbol{r}) \delta \rho\left(\boldsymbol{r}^{\prime}\right)$ ) e permutando as variáveis $r$ e $r^{\prime}$ de uma das integrais remanescentes, obtém-se que, 


$$
\delta J[\rho(\boldsymbol{r})] \approx \int d \boldsymbol{r} \delta \rho(\boldsymbol{r}) \int d \boldsymbol{r}^{\prime} \frac{\rho\left(\boldsymbol{r}^{\prime}\right)}{\left\|\boldsymbol{r}-\boldsymbol{r}^{\prime}\right\|},
$$

e portanto,

$$
\frac{\delta J[\rho(\boldsymbol{r})]}{\delta \rho(\boldsymbol{r})} \approx \int d \boldsymbol{r}^{\prime} \frac{\rho\left(\boldsymbol{r}^{\prime}\right)}{\left\|\boldsymbol{r}-\boldsymbol{r}^{\prime}\right\|}
$$

\section{B.2 Potencial externo}

Aplicando o mesmo procedimento do caso acima,

$$
\begin{aligned}
\delta V_{e n}[\rho] & =V_{e n}[\rho(\boldsymbol{r})+\delta \rho(\boldsymbol{r})]-V_{e n}[\rho(\boldsymbol{r})] \\
& =\int d \boldsymbol{r}[\rho(\boldsymbol{r})+\delta \rho(\boldsymbol{r})] v(\boldsymbol{r})-\int d \boldsymbol{r} \rho(\boldsymbol{r}) v(\boldsymbol{r}) \\
& =\int d \boldsymbol{r} \delta \rho(\boldsymbol{r}) v(\boldsymbol{r}) .
\end{aligned}
$$

Logo,

$$
\frac{\delta V_{e n}[\rho]}{\delta \rho(\boldsymbol{r})}=v(\boldsymbol{r}) .
$$

\section{B.3 Energia cinética do gás de elétrons não intera- gentes}

O cálculo da energia cinética é melhor descrito em termos da variação explícita dos orbitais. Os elementos de matriz do operador $T_{s}$ são dados por,

$$
T_{s}[\rho]=\sum_{i, j=1}^{N}\left\langle\psi_{j}\left|\left(-\frac{\nabla^{2}}{2}\right)\right| \psi_{j}\right\rangle .
$$

Tomando o diferencial com respeito ao $i$-ésimo spin-orbital e por simplicidade, assumindo que os orbitais $\psi_{i}(\boldsymbol{r})$ são reais, 


$$
\begin{aligned}
\delta T_{s}[\rho]= & T_{s}\left[\psi_{1}, \ldots, \psi_{i}+\delta \psi_{i}, \ldots, \psi_{N}\right]-T_{s}\left[\psi_{1}, \ldots, \psi_{N}\right] \\
= & -\frac{1}{2} \sum_{j \neq i}^{N} \int d \boldsymbol{r} \psi_{j} \nabla^{2} \psi_{j}-\frac{1}{2} \int d \boldsymbol{r}\left[\psi_{i}+\delta \psi_{i}\right] \nabla^{2}\left[\psi_{i}+\delta \psi_{i}\right] \\
& +\frac{1}{2} \sum_{j=1}^{N} \int d \boldsymbol{r} \psi_{j} \nabla^{2} \psi_{j} \\
= & -\frac{1}{2} \int d \boldsymbol{r}\left[\psi_{i}+\delta \psi_{i}\right] \nabla^{2}\left[\psi_{i}+\delta \psi_{i}\right]+\frac{1}{2} \int d \boldsymbol{r} \psi_{i} \nabla^{2} \psi_{i}
\end{aligned}
$$

Desprezando a contribuição de ordem superior e utilizando a definição do Laplaciano,

$$
\nabla^{2} \psi_{i}=\nabla \cdot \nabla \psi_{i}
$$

segue que,

$$
\begin{aligned}
\delta T_{s}[\rho] & =-\frac{1}{2} \int d \boldsymbol{r} \psi_{i} \nabla^{2} \delta \psi_{i}-\frac{1}{2} \int d \boldsymbol{r} \delta \psi_{i} \nabla^{2} \psi_{i}, \\
& =-\frac{1}{2} \int d \boldsymbol{r} \psi_{i} \nabla \cdot \nabla \psi_{i}-\frac{1}{2} \int d \boldsymbol{r} \delta \psi_{i} \nabla^{2} \psi_{i} .
\end{aligned}
$$

Utilizando duas vezes a técnica de integração por partes na primeira integral,

$$
\begin{aligned}
\int d \boldsymbol{r} \psi_{i} \nabla \cdot \nabla \delta \psi_{i} & =\left.\psi_{i} \nabla \delta \psi_{i}\right|_{\mathbb{R}^{3}}-\int d \boldsymbol{r} \nabla \delta \psi_{i} \nabla \psi_{i} \\
& =\left.\psi_{i} \nabla \delta \psi_{i}\right|_{\mathbb{R}^{3}}-\left.\nabla \psi_{i} \delta \psi_{i}\right|_{\mathbb{R}^{3}}+\int d \boldsymbol{r} \nabla^{2} \psi_{i} \delta \psi_{i}
\end{aligned}
$$

Como os spin-orbitais representam autoestados físicos, eles moram em um espaço de Hilbert e portanto são quadraticamente integráveis. Nos limites da região de integração em que $r \rightarrow \pm \infty$ eles tendem a zero, sobrando apenas a integral. Portanto, a derivada do termo cinético é dada por,

$$
\frac{\delta T_{s}[\rho(\boldsymbol{r})]}{\delta \psi_{i}(\boldsymbol{r})} \approx 2\left(-\frac{1}{2} \nabla^{2} \psi_{i}(\boldsymbol{r})\right)=-\nabla^{2} \psi_{i}(\boldsymbol{r})
$$




\section{B.4 Vínculo de ortogonalidade}

De maneira análoga aos casos anteriores, o diferencial do vínculo resulta em,

$$
\frac{\delta}{\delta \psi_{i}^{*}(\boldsymbol{r})}\left\{-\sum_{i, j=1}^{N} \epsilon_{i j}\left(\int d \boldsymbol{r} \psi_{i}^{*}(\boldsymbol{r}) \psi_{j}(\boldsymbol{r})-\delta_{i j}\right)\right\}=-\sum_{j=1}^{N} \epsilon_{i j} \psi_{j}(\boldsymbol{r})
$$

Retomando o cálculo de (B.1) e tendo em vista a relação (B.2), ao substituir as expressões (B.3), (B.4), (B.5) e (B.6) nas respectivas derivadas, segue que,

$$
\begin{array}{r}
\frac{\delta}{\delta \psi_{i}^{*}(\boldsymbol{r})}\left\{T_{s}[\rho(\boldsymbol{r})]+J[\rho(\boldsymbol{r})]+\int d \boldsymbol{r} \rho(\boldsymbol{r}) v(\boldsymbol{r})+E_{x c}[\rho(\boldsymbol{r})]-\sum_{i, j=1}^{N} \epsilon_{i j}\left(\int d \boldsymbol{r} \psi_{i}^{*}(\boldsymbol{r}) \psi_{j}(\boldsymbol{r})-\delta_{i j}\right)\right\}=0, \\
\left(-\nabla^{2}+2 \int d \boldsymbol{r}^{\prime} \frac{\rho\left(\boldsymbol{r}^{\prime}\right)}{\left\|\boldsymbol{r}-\boldsymbol{r}^{\prime}\right\|}+2 v(\boldsymbol{r})+2 v_{x c}(\boldsymbol{r})\right) \psi_{i}(\boldsymbol{r})-\sum_{j=1}^{N} \epsilon_{i j} \psi_{j}(\boldsymbol{r})=0,
\end{array}
$$

onde $v_{x c}$ é a derivada funcional de $E_{x c}$ com relação a densidade, dada pela expressão (2.21). Como o operador acima é hermitiano, é possível aplicar uma transformação unitária tal que a matriz $\epsilon_{i j}$ seja diagonalizada. Dividindo ambos os lados da igualdade por 2 antes de aplicar a transformação, obtemos as equações de Kohn-Sham na forma usual,

$$
\left(-\frac{\nabla^{2}}{2}+\int d \boldsymbol{r}^{\prime} \frac{\rho\left(\boldsymbol{r}^{\prime}\right)}{\left\|\boldsymbol{r}-\boldsymbol{r}^{\prime}\right\|}+v(\boldsymbol{r})+v_{x c}(\boldsymbol{r})\right) \psi_{k}(\boldsymbol{r})=\epsilon_{k} \psi_{k}(\boldsymbol{r}) .
$$




\section{Apêndice $\mathrm{C}$}

\section{Elementos do funcional $\omega$ B97X-D}

De acordo com os esquemas apresentados em [50] e [52], a energia total de correlação dinâmica é separada em duas componentes, uma de spins opostos e outra de spins paralelos,

$$
E_{c}^{\mathrm{B} 97}=\sum_{\sigma} E_{c \sigma \sigma}^{B 97}+E_{c \alpha \beta}^{B 97} .
$$

A tabela abaixo contém as contribuições de cada caso.

\begin{tabular}{|c|c|c|}
\hline & Projeções de spin iguais $(\sigma \sigma)$ & Projeções de spins opostos $(\alpha \beta)$ \\
\hline$E_{c_{--}}^{B 97}$ & $\int d \boldsymbol{r} e_{c \sigma \sigma}^{L S D A}\left(\rho_{\sigma}\right) g_{c \sigma \sigma}\left(s_{\sigma}^{2}\right)$ & $\int d \boldsymbol{r} e_{c \sigma \beta}^{L S D A}\left(\rho_{\alpha}, \rho_{\beta}\right) g_{c \alpha \beta}\left(s_{\text {avg }}^{2}\right)$ \\
\hline$g_{c_{--}}\left(s^{2}\right)$ & $\sum_{i=0}^{m} c_{c \sigma \sigma, i} u_{c \sigma \sigma}^{i}$ & $\sum_{i=0}^{m} c_{c \alpha \beta, i} u_{c \alpha \beta}^{i}$ \\
\hline$u_{c_{--}}$ & $\frac{\gamma_{c \sigma \sigma} s_{\sigma}^{2}}{1+\gamma_{c \sigma \sigma} s_{\sigma}^{2}}$ & $\frac{\gamma_{c \alpha \beta} s_{a v g}^{2}}{1+\gamma_{c \alpha \beta} s_{a v g}^{2}}$ \\
\hline$\gamma_{c--}$ & 0.2 & 0.06 \\
\hline$s_{\sigma}$ & & $\frac{\nabla \rho_{\sigma} \|}{\rho_{\sigma}^{4 / 3}}$ \\
\hline$s_{\text {avg }}$ & - & $\frac{s_{\alpha}^{2}+s_{\beta}^{2}}{2}$ \\
\hline
\end{tabular}

Tabela C.1: Contribuições para a energia total de correlação dinâmica.

As densidades de energia de correlação $e_{c \sigma \sigma}^{\mathrm{LSDA}}$ e $e_{c \alpha \beta}^{\mathrm{LSDA}}$ são derivadas da parametrização de Perdew-Wang da energia de correlação LSDA ${ }^{1}$ (Local spin-density approximation) usando a abordagem de Stoll et al. [115],

\footnotetext{
${ }^{1} \mathrm{~A}$ mudança de nomenclatura para spin-density corresponde apenas a generalização para o caso em que as densidades de cada projeção de spin são distintas, e.g. sistemas de camada aberta. Uma discussão detalhada pode ser encontrada em [115].
} 


$$
\begin{gathered}
e_{c \sigma \sigma}^{L S D A}\left(\rho_{\sigma}\right)=e_{c}^{L S D A}\left(\rho_{\sigma}, 0\right), \\
e_{c \alpha \beta}^{L S D A}\left(\rho_{\alpha}, \rho_{\beta}\right)=e_{c}^{L S D A}\left(\rho_{\alpha}, \rho_{\beta}\right)-e_{c}^{L S D A}\left(\rho_{\alpha}, 0\right)-e_{c}^{L S D A}\left(0, \rho_{\beta}\right) .
\end{gathered}
$$

Já a energia de troca é dada por,

$$
E_{x}^{\mathrm{SR}-\mathrm{B} 97}=\sum_{\sigma} \int d \boldsymbol{r} e_{x \sigma}^{\mathrm{SR}-\mathrm{B} 97}\left(\rho_{\sigma}\right) g_{x \sigma}\left(s_{\sigma}^{2}\right)
$$

com

$$
\begin{gathered}
g_{x \sigma}\left(s_{\sigma}^{2}\right)=\sum_{i=0}^{4} c_{x \sigma, i} u_{x \sigma}^{i}, \\
u_{x \sigma}=\frac{\gamma_{x \sigma} s_{\sigma}^{2}}{1+\gamma_{x \sigma} s_{\sigma}^{2}}, \\
\gamma_{x \sigma}=0.004 .
\end{gathered}
$$

Sendo a densidade de energia de troca para a projeção de spin $\sigma$ dada por,

$$
e_{x \sigma}^{\mathrm{SR}-\mathrm{B} 97}\left(\rho_{\sigma}\right)=-\frac{3}{2}\left(\frac{3}{4 \pi}\right)^{\frac{1}{3}} \rho_{\sigma}^{4 / 3}(\boldsymbol{r}) F\left(a_{\sigma, \omega}\right)=e_{x \sigma}^{\mathrm{B} 97} F\left(a_{\sigma, \omega}\right),
$$

onde $F\left(a_{\sigma, \omega}\right)$ é a função de atenuação,

$$
F\left(a_{\sigma, \omega}\right)=1-\frac{8}{3} a_{\sigma, \omega}\left[\sqrt{\pi} \operatorname{erf}\left(\frac{1}{2 a_{\sigma, \omega}}\right)-3 a_{\sigma, \omega}+4 a_{\sigma, \omega}^{3}+\left(2 a_{\sigma, \omega}-4 a_{\sigma, \omega}^{3}\right) \exp \left(-\frac{1}{4 a_{\sigma, \omega}^{2}}\right)\right],
$$

controlada pelo parâmetro adimensional,

$$
a_{\sigma, \omega} \equiv \frac{\omega}{2 k_{F \sigma}}=\frac{\omega}{2\left[6 \pi^{2} \rho_{\sigma}(\boldsymbol{r})\right]^{\frac{1}{3}}},
$$

onde $k_{F \sigma}$ é chamado de vetor de onda de Fermi local. A tabela abaixo traz os valores dos parâmetros ajustados a partir de 412 dados experimentais e cálculos teóricos [50, 51]. 


\begin{tabular}{c|c||c|c}
\hline $\mathrm{a}$ & 6.0 & $\omega$ & $0.2 a_{0}^{-1}$ \\
$c_{x}$ & 0.2222036 & $c_{x \sigma, 0}$ & 0.777964 \\
$c_{x \sigma, 1}$ & 0.661160 & $c_{x \sigma, 2}$ & 0.574541 \\
$c_{x \sigma, 3}$ & -5.256710 & $c_{x \sigma, 4}$ & 11.638600 \\
$c_{c \sigma \sigma, 0}$ & 1.000000 & $c_{c \sigma \sigma, 1}$ & -6.905390 \\
$c_{c \sigma \sigma, 2}$ & 31.334300 & $c_{c \sigma \sigma, 3}$ & -51.053300 \\
$c_{c \sigma \sigma, 4}$ & 26.442300 & $c_{c \sigma \beta, 0}$ & 1.000000 \\
$c_{c \alpha \beta, 1}$ & 1.794130 & $c_{c \alpha \beta, 2}$ & -12.047700 \\
$c_{c \alpha \beta, 3}$ & 14.084700 & $c_{c \alpha \beta, 4}$ & -8.508090 \\
\hline
\end{tabular}

Tabela C.2: Parâmetros otimizados para o funcional $\omega$ B97X-D. 


\section{Apêndice D}

\section{Operador de evolução temporal}

Como indicado, ao substituir (2.28) em (2.27), segue que,

$$
\begin{aligned}
\left(\hat{\mathcal{H}}_{0}+\hat{\mathcal{H}}_{1}(t)\right) \hat{U}\left(t, t_{0}\right) \Psi_{0} & =i \frac{\partial}{\partial t}\left(\hat{U}\left(t, t_{0}\right) \Psi_{0}\right) \\
\left(\hat{\mathcal{H}}_{0}+\hat{\mathcal{H}}_{1}(t)\right)\left(e^{-i \hat{\mathcal{H}}_{0}\left(t-t_{0}\right)} \hat{U}_{1}\left(t, t_{0}\right)\right) \Psi_{0} & =i \frac{\partial}{\partial t}\left(e^{-i \hat{\mathcal{H}}_{0}\left(t-t_{0}\right)} \hat{U}_{1}\left(t, t_{0}\right) \Psi_{0}\right) \\
\left(\hat{\mathcal{H}}_{0}+\hat{\mathcal{H}}_{1}(t)\right) e^{-i \hat{\mathcal{H}}_{0}\left(t-t_{0}\right)} \hat{U}_{1}\left(t, t_{0}\right) \Psi_{0} & =i \Psi_{0}\left[-i \hat{\mathcal{H}}_{0} e^{-i \hat{\mathcal{H}}_{0}\left(t-t_{0}\right)} \hat{U}_{1}\left(t, t_{0}\right)+e^{-i \hat{\mathcal{H}}_{0}\left(t-t_{0}\right)} \frac{\partial}{\partial t} \hat{U}_{1}\left(t, t_{0}\right)\right] \\
\hat{\mathcal{H}}_{1}(t) e^{-i \hat{\mathcal{H}}_{0}\left(t-t_{0}\right)} \hat{U}_{1}\left(t, t_{0}\right) \Psi_{0} & =i e^{-i \hat{\mathcal{H}}_{0}\left(t-t_{0}\right)} \frac{\partial}{\partial t} \hat{U}_{1}\left(t, t_{0}\right) \Psi_{0} .
\end{aligned}
$$

Aplicando $e^{i \hat{\mathcal{H}}_{0}\left(t-t_{0}\right)}$ pela esquerda, obtemos que,

$$
i \frac{\partial}{\partial t} \hat{U}_{1}\left(t, t_{0}\right)=e^{i \hat{\mathcal{H}}_{0}\left(t-t_{0}\right)} \hat{\mathcal{H}}_{1}(t) e^{-i \hat{\mathcal{H}}_{0}\left(t-t_{0}\right)} \hat{U}_{1}\left(t, t_{0}\right) .
$$

A equação acima pode ser resolvida de maneira iterativa, e a solução linear é obtida substituindo a solução de ordem zero no termo da esquerda, $\hat{U}_{1}\left(t, t_{0}\right)=1$, e integrando no tempo de $t_{0}$ até $t$,

$$
\begin{aligned}
i \int_{t_{0}}^{t} d t^{\prime} \frac{\partial}{\partial t^{\prime}} \hat{U}_{1}\left(t^{\prime}, t_{0}\right) & \simeq \int_{t_{0}}^{t} d t^{\prime} e^{i \hat{\mathcal{H}}_{0}\left(t^{\prime}-t_{0}\right)} \hat{\mathcal{H}}_{1}\left(t^{\prime}\right) e^{-i \hat{\mathcal{H}}_{0}\left(t^{\prime}-t_{0}\right)} \\
\left.i \hat{U}_{1}\left(t^{\prime}, t_{0}\right)\right|_{t_{0}} ^{t} & \simeq \int_{t_{0}}^{t} d t^{\prime} e^{i \hat{\mathcal{H}}_{0}\left(t^{\prime}-t_{0}\right)} \hat{\mathcal{H}}_{1}\left(t^{\prime}\right) e^{-i \hat{\mathcal{H}}_{0}\left(t^{\prime}-t_{0}\right)} .
\end{aligned}
$$

Multiplicando ambos os lados por $-i$ e impondo a condição inicial de que $\hat{U}_{1}\left(t_{0}, t_{0}\right)=1$, obtemos que,

$$
\hat{U}_{1}\left(t, t_{0}\right) \simeq 1-i \int_{t_{0}}^{t} d t^{\prime} e^{i \hat{\mathcal{H}}_{0}\left(t^{\prime}-t_{0}\right)} \hat{\mathcal{H}}_{1}\left(t^{\prime}\right) e^{-i \hat{\mathcal{H}}_{0}\left(t^{\prime}-t_{0}\right)},
$$

e consequentemente,

$$
\hat{U}\left(t, t_{0}\right) \simeq e^{-i \hat{\mathcal{H}}_{0}\left(t-t_{0}\right)}\left\{1-i \int_{t_{0}}^{t} d t^{\prime} e^{i \hat{\mathcal{H}}_{0}\left(t^{\prime}-t_{0}\right)} \hat{\mathcal{H}}_{1}\left(t^{\prime}\right) e^{-i \hat{\mathcal{H}}_{0}\left(t^{\prime}-t_{0}\right)}\right\}
$$




\section{Apêndice E}

\section{Cálculo da resposta linear}

Partindo do valor esperado do operador $\hat{\alpha}$ dado pela equação (2.33) e propagando a função de onda por meio do operador de evolução temporal de primeira ordem (2.32), obtemos que,

$$
\begin{aligned}
& \alpha(t)=\langle\Psi(t)|\hat{\alpha}| \Psi(t)\rangle \\
& =\left\langle\Psi_{0}\left|\hat{U}^{\dagger}\left(t, t_{0}\right) \hat{\alpha} \hat{U}\left(t, t_{0}\right)\right| \Psi_{0}\right\rangle \\
& =\left\langle\Psi_{0}\right|\left(e^{i \hat{\mathcal{H}}_{0}\left(t-t_{0}\right)}\left\{1+i \int_{t_{0}}^{t} d t^{\prime} F\left(t^{\prime}\right) \hat{\mathcal{O}}\left(t^{\prime}-t_{0}\right)\right\}\right) \hat{\alpha} \\
& \left(e^{-i \hat{\mathcal{H}}_{0}\left(t-t_{0}\right)}\left\{1-i \int_{t_{0}}^{t} d t^{\prime} F\left(t^{\prime}\right) \hat{\mathcal{O}}\left(t^{\prime}-t_{0}\right)\right\}\right)\left|\Psi_{0}\right\rangle \\
& =\left\langle\Psi_{0}\left|e^{i \hat{\mathcal{H}}_{0}\left(t-t_{0}\right)} \hat{\alpha} e^{-i \hat{\mathcal{H}}_{0}\left(t-t_{0}\right)}\right| \Psi_{0}\right\rangle-i\left\langle\Psi_{0}\left|e^{i \hat{\mathcal{H}}_{0}\left(t-t_{0}\right)} \hat{\alpha} e^{-i \hat{\mathcal{H}}_{0}\left(t-t_{0}\right)} \int_{t_{0}}^{t} d t^{\prime} F\left(t^{\prime}\right) \hat{\mathcal{O}}\left(t^{\prime}-t_{0}\right)\right| \Psi_{0}\right\rangle \\
& +i\left\langle\Psi_{0}\left|e^{i \hat{\mathcal{H}}_{0}\left(t-t_{0}\right)} \int_{t_{0}}^{t} d t^{\prime} F\left(t^{\prime}\right) \hat{\mathcal{O}}\left(t^{\prime}-t_{0}\right) \hat{\alpha} e^{-i \hat{\mathcal{H}}_{0}\left(t-t_{0}\right)}\right| \Psi_{0}\right\rangle \\
& +i\left\langle\Psi_{0}\left|e^{i \hat{\mathcal{H}}_{0}\left(t-t_{0}\right)}\left(\int_{t_{0}}^{t} d t^{\prime} F\left(t^{\prime}\right) \hat{\mathcal{O}}\left(t^{\prime}-t_{0}\right)\right) \hat{\alpha} e^{-i \hat{\mathcal{H}}_{0}\left(t-t_{0}\right)}\left(\int_{t_{0}}^{t} d t^{\prime} F\left(t^{\prime}\right) \hat{\mathcal{O}}\left(t^{\prime}-t_{0}\right)\right)\right| \Psi_{0}\right\rangle
\end{aligned}
$$

Desprezando o termo de segunda e tendo em vista que o primeiro elemento da equação acima corresponde ao termo de ordem zero, com auxílio da equação (2.31) a resposta linear assume a forma,

$$
\begin{aligned}
\alpha_{1}(t) & =\alpha(t)-\alpha_{0} \\
& =-i\left\langle\Psi_{0}\left|\left\{\hat{\alpha}\left(t-t_{0}\right) \int_{t_{0}}^{t} d t^{\prime} F\left(t^{\prime}\right) \hat{\mathcal{O}}\left(t^{\prime}-t_{0}\right)-\int_{t_{0}}^{t} \hat{\mathcal{O}}\left(t^{\prime}-t_{0}\right) \hat{\alpha}\left(t-t_{0}\right)\right\}\right| \Psi_{0}\right\rangle \\
& =-i \int_{t_{0}}^{t} d t^{\prime} F\left(t^{\prime}\right)\left\langle\Psi_{0}\left|\left\{\hat{\alpha}\left(t-t_{0}\right) \hat{\mathcal{O}}\left(t^{\prime}-t_{0}\right)-\hat{\mathcal{O}}\left(t^{\prime}-t_{0}\right) \hat{\alpha}\left(t-t_{0}\right)\right\}\right| \Psi_{0}\right\rangle \\
& =-i \int_{t_{0}}^{t} d t^{\prime} F\left(t^{\prime}\right)\left\langle\Psi_{0}\left|\left[\hat{\alpha}\left(t-t_{0}\right), \hat{\mathcal{O}}\left(t^{\prime}-t_{0}\right)\right]\right| \Psi_{0}\right\rangle .
\end{aligned}
$$

Manipulando o comutador de maneira conveniente, podemos restringir a dependência temporal para apenas um dos operadores. Utilizando a 
notação compacta $\hat{U}_{0}(t)=e^{-i \hat{\mathcal{H}}_{0} t}$, segue que,

$$
\begin{aligned}
{\left[\hat{\alpha}\left(t-t_{0}\right), \hat{\mathcal{O}}\left(t^{\prime}-t_{0}\right)\right]=} & \hat{\alpha}\left(t-t_{0}\right) \hat{\mathcal{O}}\left(t^{\prime}-t_{0}\right)-\hat{\mathcal{O}}\left(t^{\prime}-t_{0}\right) \hat{\alpha}\left(t-t_{0}\right) \\
= & \left(\hat{U}_{0}^{\dagger}\left(t-t_{0}\right) \hat{\alpha} \hat{U}_{0}\left(t-t_{0}\right)\right)\left(\hat{U}_{0}^{\dagger}\left(t^{\prime}-t_{0}\right) \hat{\mathcal{O}} \hat{U}_{0}\left(t^{\prime}-t_{0}\right)\right) \\
& -\left(\hat{U}_{0}^{\dagger}\left(t^{\prime}-t_{0}\right) \hat{\mathcal{O}} \hat{U}_{0}\left(t^{\prime}-t_{0}\right)\right)\left(\hat{U}_{0}^{\dagger}\left(t-t_{0}\right) \hat{\alpha} \hat{U}_{0}\left(t-t_{0}\right)\right) \\
= & \hat{U}_{0}^{\dagger}\left(t-t_{0}\right) \hat{\alpha} \hat{U}_{0}\left(t-t^{\prime}\right) \hat{\mathcal{O}} \hat{U}_{0}\left(t^{\prime}-t_{0}\right) \\
& -\hat{U}_{0}^{\dagger}\left(t^{\prime}-t_{0}\right) \hat{\mathcal{O}} \hat{U}^{\dagger}\left(t-t^{\prime}\right) \hat{\alpha} \hat{U}_{0}\left(t-t_{0}\right)
\end{aligned}
$$

Inserindo a identidade $\hat{U}_{0}\left(t-t^{\prime}\right) \hat{U}_{0}^{\dagger}\left(t-t^{\prime}\right)=1$ na esquerda e na direita do operador $\hat{\alpha}$, respectivamente na primeira e na segunda aparição de $\hat{\alpha}$, podemos reescrever o operador na representação de interação da forma $\hat{\alpha}\left(t-t^{\prime}\right)$, e portanto,

$$
\begin{aligned}
{\left[\hat{\alpha}\left(t-t_{0}\right), \hat{\mathcal{O}}\left(t^{\prime}-t_{0}\right)\right]=} & \hat{U}_{0}^{\dagger}\left(t^{\prime}-t_{0}\right) \hat{\alpha}\left(t-t^{\prime}\right) \hat{\mathcal{O}} \hat{U}_{0}\left(t^{\prime}-t_{0}\right) \\
& -\hat{U}_{0}^{\dagger}\left(t^{\prime}-t_{0}\right) \hat{\mathcal{O}} \hat{\alpha}\left(t-t^{\prime}\right) \hat{U}_{0}\left(t^{\prime}-t_{0}\right) \\
= & \hat{U}_{0}^{\dagger}\left(t^{\prime}-t_{0}\right)\left[\hat{\alpha}\left(t-t^{\prime}\right), \hat{\mathcal{O}}\right] \hat{U}_{0}\left(t^{\prime}-t_{0}\right) .
\end{aligned}
$$

Retomando no cálculo de $\alpha_{1}(t)$ pela equação (E.1), os operadores $\hat{U}_{0}$ atuam em $\Psi_{0}$ gerando fases complexas que se anulam e a resposta linear é dada por,

$$
\alpha_{1}(t)=-i \int_{t_{0}}^{t} d t^{\prime} F\left(t^{\prime}\right)\left\langle\Psi_{0}\left|\left[\hat{\alpha}\left(t-t^{\prime}\right), \hat{\mathcal{O}}\right]\right| \Psi_{0}\right\rangle .
$$

O termo que multiplica $F\left(t^{\prime}\right)$, chamado de função resposta linear, é formado pelos termos de primeira ordem que representam a resposta do operador $\hat{\alpha}$ frente a perturbação $F\left(t^{\prime}\right) \hat{\mathcal{O}}$. No caso de uma perturbação iniciada no instante $t=t_{0}$, a função de Heaviside $\theta\left(t-t^{\prime}\right)$ é incorporada na expressão garantindo que a causalidade seja preservada, e como $F\left(t^{\prime}\right)$ é nulo para qualquer $t^{\prime}<t_{0}$, a resposta linear se resume a expressão abaixo, 


$$
\alpha_{1}(t)=-i \int_{-\infty}^{\infty} d t^{\prime} \theta\left(t-t^{\prime}\right) F\left(t^{\prime}\right)\left\langle\Psi_{0}\left|\left[\hat{\alpha}\left(t-t^{\prime}\right), \hat{\mathcal{O}}\right]\right| \Psi_{0}\right\rangle
$$




\section{Apêndice F}

\section{Representação de Lehmann da função de resposta linear}

Considere o conjunto completo de autofunções da hamiltoniana $H_{0}$, $\left\{\left|\Psi_{n}\right\rangle\right\}$ com $n=0,1,2, \ldots$, onde $\left|\Psi_{0}\right\rangle$ é o estado fundamental de $H_{0}$ com energia $E_{0},\left|\Psi_{1}\right\rangle$ o primeiro estado excitado com energia $E_{1}$ e assim sucessivamente. Pela equação (2.37), temos que,

$$
\begin{aligned}
\tilde{\chi}_{\alpha \mathcal{O}}(\omega) & =-i \int_{-\infty}^{\infty} d \tau \theta(\tau)\left\langle\Psi_{0}|[\hat{\alpha}(\tau), \hat{\mathcal{O}}]| \Psi_{0}\right\rangle e^{i \omega \tau} \\
& =-i \int_{-\infty}^{\infty} d \tau \theta(\tau) e^{i \omega \tau}\left\langle\Psi_{0}|(\hat{\alpha}(\tau) \hat{\mathcal{O}}-\hat{\mathcal{O}} \hat{\alpha}(\tau))| \Psi_{0}\right\rangle
\end{aligned}
$$

Introduzindo entre os operadores $\hat{\alpha}$ e $\hat{\mathcal{O}}$ a relação de completeza,

$$
\sum_{i=0}^{\infty}\left|\Psi_{i}\right\rangle\left\langle\Psi_{i}\right|=1,
$$

e expressando $\hat{\alpha}(\tau)$ de acordo com a representação de interação (2.31), segue que,

$$
\begin{aligned}
\tilde{\chi}_{\alpha \mathcal{O}}(\omega)= & -i \int_{-\infty}^{\infty} d \tau \theta(\tau) e^{i \omega \tau}\left\langle\Psi_{0}\left|\left(\hat{\alpha}(\tau) \sum_{n=0}^{\infty}\left|\Psi_{n}\right\rangle\left\langle\Psi_{n}\left|\hat{\mathcal{O}}-\hat{\mathcal{O}} \sum_{n=0}^{\infty}\right| \Psi_{n}\right\rangle\left\langle\Psi_{n}\right| \hat{\alpha}(\tau)\right)\right| \Psi_{0}\right\rangle \\
= & -i \int_{-\infty}^{\infty} d \tau \theta(\tau) e^{i \omega \tau} \sum_{n=0}^{\infty}\left\{\left\langle\Psi_{0}\left|e^{i \hat{\mathcal{H}}_{0} \tau} \hat{\alpha} e^{-i \hat{\mathcal{H}}_{0} \tau}\right| \Psi_{n}\right\rangle\left\langle\Psi_{n}|\hat{\mathcal{O}}| \Psi_{0}\right\rangle\right. \\
& \left.-\left\langle\Psi_{0}|\hat{\mathcal{O}}| \Psi_{n}\right\rangle\left\langle\Psi_{n}\left|e^{i \hat{\mathcal{H}}_{0} \tau} \hat{\alpha} e^{-i \hat{\mathcal{H}}_{0} \tau}\right| \Psi_{0}\right\rangle\right\} \\
= & -i \int_{-\infty}^{\infty} d \tau \theta(\tau) e^{i \omega \tau} \sum_{n=0}^{\infty}\left\{e^{i\left(E_{0}-E_{n}\right) \tau}\left\langle\Psi_{0}|\hat{\alpha}| \Psi_{n}\right\rangle\left\langle\Psi_{n}|\hat{\mathcal{O}}| \Psi_{0}\right\rangle\right. \\
& \left.-e^{i\left(E_{n}-E_{0}\right) \tau}\left\langle\Psi_{0}|\hat{\mathcal{O}}| \Psi_{n}\right\rangle\left\langle\Psi_{n}|\hat{\alpha}| \Psi_{0}\right\rangle\right\}
\end{aligned}
$$

Quando $n=0$ as exponenciais são iguais a 1 e os termos se anulam, portanto podemos escrever a somatória a partir de $n=1$. Definindo 
$\Omega_{n} \equiv E_{n}-E_{0}$ e utilizando a representação integral da função de Heaviside,

$$
\theta(\tau)=\lim _{\eta \rightarrow 0+} \frac{i}{2 \pi} \int_{-\infty}^{\infty} d \omega^{\prime} \frac{e^{-i \omega^{\prime} \tau}}{\omega^{\prime}+i \eta},
$$

a função de resposta linear assume a forma,

$$
\begin{aligned}
\tilde{\chi}_{\alpha \mathcal{O}}(\omega)= & -i \sum_{n=1}^{\infty} \int_{-\infty}^{\infty} d \tau\left(\lim _{\eta \rightarrow 0+} \frac{i}{2 \pi} \int_{-\infty}^{\infty} d \omega^{\prime} \frac{e^{-i \omega^{\prime} \tau}}{\omega^{\prime}+i \eta}\right) e^{i \omega \tau}\left\{e^{-i \Omega_{n} \tau}\left\langle\Psi_{0}|\hat{\alpha}| \Psi_{n}\right\rangle\left\langle\Psi_{n}\right| \ldots\right\} \\
= & \frac{1}{2 \pi} \lim _{\eta \rightarrow 0+} \sum_{n=1}^{\infty} \int_{-\infty}^{\infty} d \omega^{\prime} \frac{1}{\omega^{\prime}+i \eta}\left\{\left\langle\Psi_{0}|\hat{\alpha}| \Psi_{n}\right\rangle\left\langle\Psi_{n}|\hat{\mathcal{O}}| \Psi_{0}\right\rangle \int_{-\infty}^{\infty} d \tau e^{i\left(\omega-\omega^{\prime}-\Omega_{n}\right) \tau}\right. \\
& \left.-\left\langle\Psi_{0}|\hat{\mathcal{O}}| \Psi_{n}\right\rangle\left\langle\Psi_{n}|\hat{\alpha}| \Psi_{0}\right\rangle \int_{-\infty}^{\infty} d \tau e^{i\left(\omega-\omega^{\prime}+\Omega_{n}\right) \tau}\right\} .
\end{aligned}
$$

Utilizando novamente da relação de ortogonalidade das exponenciais, as integrais em $\tau$ se reduzem a deltas de Dirac. As deltas são simétricas frente a operações de paridade, $\delta(x)=\delta(-x)$ e satisfazem a propriedade de filtragem (2.8),

$$
\int_{-\infty}^{\infty} d x \delta(x-a) f(x)=f(a)
$$

onde $f(x)$ é uma função contínua em $x=a$. Assim sendo, a função de resposta linear pode ser expressa na chamada representação de Lehmann,

$$
\begin{aligned}
\tilde{\chi}_{\alpha \mathcal{O}}(\omega)= & \frac{1}{2 \pi} \lim _{\eta \rightarrow 0+} \sum_{n=1}^{\infty} \int_{-\infty}^{\infty} d \omega^{\prime} \frac{1}{\omega^{\prime}+i \eta}\left\{\left\langle\Psi_{0}|\hat{\alpha}| \Psi_{n}\right\rangle\left\langle\Psi_{n}|\hat{\mathcal{O}}| \Psi_{0}\right\rangle 2 \pi \delta\left(\omega-\omega^{\prime}-\Omega_{n}\right)\right. \\
& \left.-\left\langle\Psi_{0}|\hat{\mathcal{O}}| \Psi_{n}\right\rangle\left\langle\Psi_{n}|\hat{\alpha}| \Psi_{0}\right\rangle 2 \pi \delta\left(\omega-\omega^{\prime}+\Omega_{n}\right)\right\} \\
= & \lim _{\eta \rightarrow 0+} \sum_{n=1}^{\infty}\left\{\left\langle\Psi_{0}|\hat{\alpha}| \Psi_{n}\right\rangle\left\langle\Psi_{n}|\hat{\mathcal{O}}| \Psi_{0}\right\rangle \int_{-\infty}^{\infty} d \omega^{\prime} \frac{\delta\left(\omega^{\prime}-\omega+\Omega_{n}\right)}{\omega^{\prime}+i \eta}\right. \\
& \left.-\left\langle\Psi_{0}|\hat{\mathcal{O}}| \Psi_{n}\right\rangle\left\langle\Psi_{n}|\hat{\alpha}| \Psi_{0}\right\rangle \int_{-\infty}^{\infty} d \omega^{\prime} \frac{\delta\left(\omega^{\prime}-\omega-\Omega_{n}\right)}{\omega^{\prime}+i \eta}\right\} \\
= & \lim _{\eta \rightarrow 0+} \sum_{n=1}^{\infty}\left\{\frac{\left\langle\Psi_{0}|\hat{\alpha}| \Psi_{n}\right\rangle\left\langle\Psi_{n}|\hat{\mathcal{O}}| \Psi_{0}\right\rangle}{\omega-\Omega_{n}+i \eta}-\frac{\left\langle\Psi_{0}|\hat{\mathcal{O}}| \Psi_{n}\right\rangle\left\langle\Psi_{n}|\hat{\alpha}| \Psi_{0}\right\rangle}{\omega+\Omega_{n}+i \eta}\right\}
\end{aligned}
$$




\section{Apêndice G}

\section{Vínculos entre respostas lineares dos sistemas real e de Kohn-Sham}

Neste apêndice são obtidas três equações, (2.41) que relaciona as funções de resposta linear dos sistemas real e de KS, a expressão da correção de primeira ordem da densidade (2.42) e $\chi_{s}$ no espaço recíproco, dada por (2.43). Para verificar a primeira delas, expandimos cada um dos três termos do potencial efetivo (2.40) em contribuições de diferentes ordens de grandeza, tais que o potencial efetivo linearizado é dado por,

$$
v_{s 1}[\rho](\boldsymbol{r}, t)=v_{1}(\boldsymbol{r}, t)+\int d^{3} r^{\prime} \frac{\rho_{1}\left(\boldsymbol{r}^{\prime}, t\right)}{\left\|\boldsymbol{r}-\boldsymbol{r}^{\prime}\right\|}+v_{x c 1}(\boldsymbol{r}, t),
$$

onde o potencial de correlação e troca linearizado é,

$$
v_{x c 1}(\boldsymbol{r}, t)=\left.\int d t^{\prime} \int d^{3} r^{\prime} \frac{\delta v_{x c}[\rho](\boldsymbol{r}, t)}{\delta \rho\left(\boldsymbol{r}^{\prime}, t^{\prime}\right)}\right|_{\rho_{0}(\boldsymbol{r})} \rho_{1}\left(\boldsymbol{r}^{\prime}, t^{\prime}\right) .
$$

De maneira análoga a equação (2.39), a correção de primeira ordem na densidade pode ser expressa em termos de $v_{s 1}$ e $\chi_{s}$,

$$
\rho_{1}(\boldsymbol{r}, t)=\int d t^{\prime} \int d^{3} r^{\prime} \chi_{s}\left(\boldsymbol{r}, \boldsymbol{r}^{\prime}, t-t^{\prime}\right) v_{s 1}\left(\boldsymbol{r}^{\prime}, t^{\prime}\right)
$$

Substituindo (G.1) e (G.2) na expressão acima, segue que, 


$$
\begin{aligned}
\rho_{1}(\boldsymbol{r}, t)= & \int d t^{\prime} \int d^{3} r^{\prime} \chi_{s}\left(\boldsymbol{r}, \boldsymbol{r}^{\prime}, t-t^{\prime}\right)\left[v_{1}\left(\boldsymbol{r}^{\prime}, t^{\prime}\right)+\int d^{3} x \frac{\rho_{1}\left(\boldsymbol{x}, t^{\prime}\right)}{\left\|\boldsymbol{r}^{\prime}-\boldsymbol{x}\right\|}+\right. \\
& \left.+\int d \tilde{t} \int d^{3} x f_{x c}\left(\boldsymbol{x}, \boldsymbol{r}^{\prime}, \tilde{t}, t^{\prime}\right) \rho_{1}(\boldsymbol{x}, \tilde{t})\right] \\
= & \int d t^{\prime} \int d^{3} r^{\prime} \chi_{s}\left(\boldsymbol{r}, \boldsymbol{r}^{\prime}, t-t^{\prime}\right)\left[v_{1}\left(\boldsymbol{r}^{\prime}, t^{\prime}\right)+\int d \tilde{t} \int d^{3} x\left\{\frac{\delta\left(t^{\prime}-\tilde{t}\right)}{\left\|\boldsymbol{r}^{\prime}-\boldsymbol{x}\right\|}\right.\right. \\
& \left.\left.+f_{x c}\left(\boldsymbol{r}^{\prime}, \boldsymbol{x}, t^{\prime}, \tilde{t}\right)\right\} \rho_{1}(\boldsymbol{x}, \tilde{t})\right] .
\end{aligned}
$$

Comparando o resultado acima com a resposta do sistema real por meio da substituição da equação (2.39) em ambos os lados da igualdade,

$$
\begin{aligned}
\int d t^{\prime} \int d^{3} r^{\prime} \chi\left(\boldsymbol{r}, \boldsymbol{r}^{\prime}, t-t^{\prime}\right) v_{1}\left(\boldsymbol{r}^{\prime}, t^{\prime}\right)= & \int d t^{\prime} \int d^{3} r^{\prime} \chi_{s}\left(\boldsymbol{r}, \boldsymbol{r}^{\prime}, t-t^{\prime}\right)\left[v_{1}\left(\boldsymbol{r}^{\prime}, t^{\prime}\right)+\int d \tilde{t} \int d^{3} x\left\{\frac{\delta\left(t^{\prime}-\tilde{t}\right)}{\left\|\boldsymbol{r}^{\prime}-\boldsymbol{x}\right\|}\right.\right. \\
& \left.\left.+f_{x c}\left(\boldsymbol{r}^{\prime}, \boldsymbol{x}, t^{\prime}, \tilde{t}\right)\right\} \int d \tilde{t}^{\prime} \int d^{3} x^{\prime} \chi\left(\boldsymbol{x}, \boldsymbol{x}^{\prime}, \tilde{t}-\tilde{t}^{\prime}\right) v_{1}\left(\boldsymbol{x}^{\prime}, \tilde{t}^{\prime}\right)\right],
\end{aligned}
$$

podemos observar que como a expressão é válida para qualquer perturbação externa $v_{1}$, as funções de resposta linear se relacionam por meio da identidade (2.41),

$$
\begin{aligned}
\chi\left(\boldsymbol{r}, \boldsymbol{r}^{\prime}, t-t^{\prime}\right)= & \chi_{s}\left(\boldsymbol{r}, \boldsymbol{r}^{\prime}, t-t^{\prime}\right)+\int d \tilde{t} \int d^{3} x \int d \tilde{t}^{\prime} \int d^{3} x^{\prime}\left\{\frac{\delta\left(\tilde{t}-\tilde{t}^{\prime}\right)}{\left\|\boldsymbol{x}-\boldsymbol{x}^{\prime}\right\|}+\right. \\
& \left.+f_{x c}\left(\boldsymbol{x}, \boldsymbol{x}^{\prime}, \tilde{t}-\tilde{t}^{\prime}\right)\right\} \chi\left(\boldsymbol{x}^{\prime}, \boldsymbol{r}^{\prime}, \tilde{t}^{\prime}-t^{\prime}\right) .
\end{aligned}
$$

Para determinar $\rho_{1}(\boldsymbol{r}, \omega)$ partimos de (G.3) e realizamos a transformada de Fourier em todas as funções envolvidas que apresentam dependência temporal, ${ }^{1}$

$$
\begin{aligned}
\int_{-\infty}^{\infty} \frac{d \omega}{2 \pi} \rho_{1}(\boldsymbol{r}, \omega) e^{-i \omega t}= & \int d t^{\prime} \int d^{3} r^{\prime}\left(\int_{-\infty}^{\infty} \frac{d \omega}{2 \pi} \chi_{s}\left(\boldsymbol{r}, \boldsymbol{r}^{\prime}, \omega\right) e^{-i \omega\left(t-t^{\prime}\right)}\right)\left[\int_{-\infty}^{\infty} \frac{d \omega^{\prime}}{2 \pi} v_{1}\left(\boldsymbol{r}^{\prime}, \omega^{\prime}\right) e^{-i \omega^{\prime} t^{\prime}}+\right. \\
& +\int d \tilde{t} \int d^{3} x\left\{\int_{-\infty}^{\infty} \frac{d \omega^{\prime \prime}}{2 \pi} \frac{\delta\left(\omega^{\prime \prime}\right)}{\left\|\boldsymbol{r}^{\prime}-\boldsymbol{x}\right\|} e^{-i \omega^{\prime \prime}\left(t^{\prime}-\tilde{t}\right)}+\int_{-\infty}^{\infty} \frac{d \omega^{\prime \prime \prime}}{2 \pi} f_{x c}\left(\boldsymbol{r}^{\prime}, \boldsymbol{x}, \omega^{\prime \prime \prime}\right) e^{-i \omega^{\prime \prime \prime}\left(t^{\prime}-\tilde{t}\right)}\right\} \\
& \left.\int_{-\infty}^{\infty} \frac{d \tilde{\omega}}{2 \pi} \rho_{1}(\boldsymbol{x}, \tilde{\omega}) e^{-i \tilde{\omega} \tilde{t}}\right]
\end{aligned}
$$

\footnotetext{
${ }^{1}$ Explicitamente, as funções transformadas são $\rho_{1}(\boldsymbol{r}, t), \chi_{s}\left(\boldsymbol{r}, \boldsymbol{r}^{\prime}, t-t^{\prime}\right), v_{1}\left(\boldsymbol{r}^{\prime}, t^{\prime}\right), \delta\left(t^{\prime}-\tilde{t}\right), f_{x c}\left(\boldsymbol{r}^{\prime}, \boldsymbol{x}, t^{\prime}, \tilde{t}\right)$ e $\rho_{1}(\boldsymbol{x}, \tilde{t})$.
} 
Explorando a relação de ortogonalidade das funções exponenciais algumas deltas aparecem, e através da propriedade de filtragem (2.8) é possível simplificar a expressão acima,

$$
\begin{aligned}
& \int_{-\infty}^{\infty} \frac{d \omega}{2 \pi} \rho_{1}(\boldsymbol{r}, \omega) e^{-i \omega t}=\int d^{3} r^{\prime} \int_{-\infty}^{\infty} \frac{d \omega}{2 \pi} \int_{-\infty}^{\infty} \frac{d \omega^{\prime}}{2 \pi} \chi_{s}\left(\boldsymbol{r}, \boldsymbol{r}^{\prime}, \omega\right) v_{1}\left(\boldsymbol{r}^{\prime}, \omega^{\prime}\right) e^{-i \omega t} \overbrace{\int_{-\infty}^{\infty} d t^{\prime} e^{i t^{\prime}\left(\omega-\omega^{\prime}\right)}}^{2 \pi \delta\left(\omega-\omega^{\prime}\right)}+ \\
& +\int d^{3} r^{\prime} \int d^{3} x \int \frac{d \omega}{2 \pi} \chi_{s}\left(\boldsymbol{r}, \boldsymbol{r}^{\prime}, \omega\right) \int_{-\infty}^{\infty} \frac{d \omega^{\prime \prime}}{2 \pi} \frac{\delta\left(\omega^{\prime \prime}\right)}{\left\|\boldsymbol{r}^{\prime}-\boldsymbol{x}\right\|}\left[\int_{-\infty}^{\infty} d t^{\prime} e^{i t^{\prime}\left(\omega-\omega^{\prime \prime}\right)}\right] \\
& {\left[\int_{-\infty}^{\infty} d \tilde{t} e^{i \tilde{t}\left(\omega^{\prime \prime}-\tilde{\omega}\right)}\right] e^{-i \omega t} \int_{-\infty}^{\infty} \frac{d \tilde{\omega}}{2 \pi} \rho_{1}(\boldsymbol{x}, \tilde{\omega})+\int d^{3} r^{\prime} \int d^{3} x \int \frac{d \omega}{2 \pi} \chi_{s}\left(\boldsymbol{r}, \boldsymbol{r}^{\prime}, \omega\right)} \\
& \int_{-\infty}^{\infty} \frac{d \omega^{\prime \prime \prime}}{2 \pi} f_{x c}\left(\boldsymbol{r}^{\prime}, \boldsymbol{x}, \omega^{\prime \prime \prime}\right)\left[\int_{-\infty}^{\infty} d t^{\prime} e^{i t^{\prime}\left(\omega-\omega^{\prime \prime \prime}\right)}\right]\left[\int_{-\infty}^{\infty} d \tilde{d} e^{i \tilde{t}\left(\omega^{\prime \prime \prime}-\tilde{\omega}\right)}\right] e^{-i \omega t} \int_{-\infty}^{\infty} \frac{d \tilde{\omega}}{2 \pi} \rho_{1}(\boldsymbol{x}, \tilde{\omega}) \\
& =\int d^{3} r^{\prime} \int_{-\infty}^{\infty} \frac{d \omega}{2 \pi} \chi_{s}\left(\boldsymbol{r}, \boldsymbol{r}^{\prime}, \omega\right) e^{-i \omega t} \underbrace{\int_{-\infty}^{\infty} d \omega^{\prime} v_{1}\left(\boldsymbol{r}^{\prime}, \omega^{\prime}\right) \delta\left(\omega-\omega^{\prime}\right)}_{v_{1}\left(\boldsymbol{r}^{\prime}, \omega\right)}+ \\
& +\int d^{3} r^{\prime} \int d^{3} x \int \frac{d \omega}{2 \pi} \chi_{s}\left(\boldsymbol{r}, \boldsymbol{r}^{\prime}, \omega\right) \int_{-\infty}^{\infty} \frac{d \omega^{\prime \prime}}{2 \pi} \frac{\delta\left(\omega^{\prime \prime}\right)}{\left\|\boldsymbol{r}^{\prime}-\boldsymbol{x}\right\|} 2 \pi \delta\left(\omega-\omega^{\prime \prime}\right) 2 \pi \delta\left(\omega^{\prime \prime}-\tilde{\omega}\right) e^{-i \omega t} \\
& \int_{-\infty}^{\infty} \frac{d \omega}{2 \pi} \rho_{1}(\boldsymbol{x}, \tilde{\omega})+\int d^{3} r^{\prime} \int d^{3} x \int \frac{d \omega}{2 \pi} \chi_{s}\left(\boldsymbol{r}, \boldsymbol{r}^{\prime}, \omega\right) \int_{-\infty}^{\infty} \frac{d \omega^{\prime \prime \prime}}{2 \pi} f_{x c}\left(\boldsymbol{r}^{\prime}, \boldsymbol{x}, \omega^{\prime \prime \prime}\right) 2 \pi \delta\left(\omega-\omega^{\prime \prime \prime}\right) \\
& 2 \pi \delta\left(\omega^{\prime \prime \prime}-\tilde{\omega}\right) e^{-i \omega t} \int_{-\infty}^{\infty} \frac{d \tilde{\omega}}{2 \pi} \rho_{1}(\boldsymbol{x}, \tilde{\omega}) \\
& =\int d^{3} r^{\prime} \int_{-\infty}^{\infty} \frac{d \omega}{2 \pi} \chi_{s}\left(\boldsymbol{r}, \boldsymbol{r}^{\prime}, \omega\right) v_{1}(\boldsymbol{r}, \omega) e^{-i \omega t}+ \\
& +\int d^{3} r^{\prime} \int d^{3} x \int \frac{d \omega}{2 \pi} \chi_{s}\left(\boldsymbol{r}, \boldsymbol{r}^{\prime}, \omega\right) \int_{-\infty}^{\infty} d \omega^{\prime \prime} \frac{\delta\left(\omega^{\prime \prime}\right)}{\left\|\boldsymbol{r}^{\prime}-\boldsymbol{x}\right\|} \delta\left(\omega-\omega^{\prime \prime}\right) e^{-i \omega t} \rho_{1}\left(\boldsymbol{x}, \omega^{\prime \prime}\right)+ \\
& +\int d^{3} r^{\prime} \int d^{3} x \int \frac{d \omega}{2 \pi} \chi_{s}\left(\boldsymbol{r}, \boldsymbol{r}^{\prime}, \omega\right) \int_{-\infty}^{\infty} d \omega^{\prime \prime \prime} f_{x c}\left(\boldsymbol{r}^{\prime}, \boldsymbol{x}, \omega^{\prime \prime \prime}\right) \delta\left(\omega-\omega^{\prime \prime \prime}\right) e^{-i \omega t} \rho_{1}\left(\boldsymbol{x}, \omega^{\prime \prime \prime}\right) \\
& =\int d^{3} r^{\prime} \int_{-\infty}^{\infty} \frac{d \omega}{2 \pi} \chi_{s}\left(\boldsymbol{r}, \boldsymbol{r}^{\prime}, \omega\right) v_{1}(\boldsymbol{r}, \omega) e^{-i \omega t}+ \\
& \int d^{3} r^{\prime} \int d^{3} x \int \frac{d \omega}{2 \pi} \chi_{s}\left(\boldsymbol{r}, \boldsymbol{r}^{\prime}, \omega\right) \frac{\delta(\omega)}{\left\|\boldsymbol{r}^{\prime}-\boldsymbol{x}\right\|} e^{-i \omega t} \rho_{1}(\boldsymbol{x}, \omega)+ \\
& \int d^{3} r^{\prime} \int d^{3} x \int \frac{d \omega}{2 \pi} \chi_{s}\left(\boldsymbol{r}, \boldsymbol{r}^{\prime}, \omega\right) f_{x c}\left(\boldsymbol{r}^{\prime}, \boldsymbol{x}, \omega\right) e^{-i \omega t} \rho_{1}(\boldsymbol{x}, \omega) \\
& =\int_{-\infty}^{\infty} \frac{d \omega}{2 \pi} \int d^{3} r^{\prime}\left\{\chi_{s}\left(\boldsymbol{r}, \boldsymbol{r}^{\prime}, \omega\right)\left[v_{1}(\boldsymbol{r}, \omega)+\left(\int d^{3} x \frac{\delta(\omega)}{\left\|\boldsymbol{r}^{\prime}-\boldsymbol{x}\right\|}+f_{x c}\left(\boldsymbol{r}^{\prime}, \boldsymbol{x}, \omega\right)\right) \rho_{1}(\boldsymbol{x}, \omega)\right]\right\} e^{-i \omega t} .
\end{aligned}
$$

Comparando o lado esquerdo com o direito chegamos na equação (2.42),

$$
\rho_{1}(\boldsymbol{r}, \omega)=\int d^{3} r^{\prime} \chi_{s}\left(\boldsymbol{r}, \boldsymbol{r}^{\prime}, \omega\right)\left[v_{1}\left(\boldsymbol{r}^{\prime}, \omega\right)+\int d^{3} x\left\{\frac{\delta(\omega)}{\left\|\boldsymbol{r}^{\prime}-\boldsymbol{x}\right\|}+f_{x c}\left(\boldsymbol{r}^{\prime}, \boldsymbol{x}, \omega\right)\right\} \rho_{1}(\boldsymbol{x}, \omega)\right] .
$$

A última parte pendente consiste em obter a expressão para $\chi_{s}$, e para isso partimos da representação de Lehmann da função de resposta linear (2.38), 


$$
\chi_{\rho \rho}(\omega)=\lim _{\eta \rightarrow 0+} \sum_{n=1}^{\infty}\left\{\frac{\left\langle\Psi_{0}|\hat{\rho}(\boldsymbol{r})| \Psi_{n}\right\rangle\left\langle\Psi_{n}\left|\hat{\rho}\left(\boldsymbol{r}^{\prime}\right)\right| \Psi_{0}\right\rangle}{\omega-\Omega_{n}+i \eta}-\frac{\left\langle\Psi_{0}\left|\hat{\rho}\left(\boldsymbol{r}^{\prime}\right)\right| \Psi_{n}\right\rangle\left\langle\Psi_{n}|\hat{\rho}(\boldsymbol{r})| \Psi_{0}\right\rangle}{\omega+\Omega_{n}+i \eta}\right\},
$$

já adaptada para a resposta do tipo densidade-densidade. No caso do sistema não interagente, os autoestados globais $\left|\Psi_{n}\right\rangle$ são determinados a partir dos orbitais de Kohn-Sham, $\left|\phi_{n}\right\rangle$. O estado fundamental $\left|\Psi_{0}\right\rangle$ é dado por um determinante de Slater da forma (2.14) enquanto os estados excitados são construídos a partir de excitações simples entre orbitais de KS. ${ }^{2}$ Sendo $\mathcal{A}$ o operador de antissimetrização, ao incorporar as contribuições de spin seguindo a notação da Seção 2.2, o estado fundamental,

$$
\Psi_{0}\left(\boldsymbol{r}_{1}, \boldsymbol{r}_{2} \ldots \boldsymbol{r}_{\frac{N}{2}}\right)=\mathcal{A}\left[\psi_{1}\left(\boldsymbol{r}_{1}\right) \psi_{2}\left(\boldsymbol{r}_{2}\right) \ldots \psi_{l}\left(\boldsymbol{r}_{i}\right) \ldots \psi_{\frac{N}{2}}\left(\boldsymbol{r}_{\frac{N}{2}}\right)\right],
$$

é formado pelos $\frac{N}{2}$ orbitais de KS de menor energia, enquanto um estado excitado,

$$
\Psi_{m}\left(\boldsymbol{r}_{1}, \boldsymbol{r}_{2} \ldots \boldsymbol{r}_{\frac{N}{2}}\right)=\mathcal{A}\left[\psi_{1}\left(\boldsymbol{r}_{1}\right) \psi_{2}\left(\boldsymbol{r}_{2}\right) \ldots \psi_{k}\left(\boldsymbol{r}_{i}\right) \ldots \psi_{\frac{N}{2}}\left(\boldsymbol{r}_{\frac{N}{2}}\right)\right]
$$

é fruto da excitação do $i$-ésimo elétron que ocupa o orbital ligado $l$ para o orbital excitado $k$. Uma vez que o operador de densidade é dado por,

$$
\hat{\rho}(\boldsymbol{r})=\sum_{j=1}^{N} \delta\left(\boldsymbol{r}-\boldsymbol{r}_{j}\right),
$$

onde $N$ é o número de elétrons, os elementos de matriz presentes na expressão da resposta linear são reduzidos a produtos de orbitais. Na notação de Dirac,

$$
\begin{aligned}
\left\langle\Psi_{0}|\hat{\rho}(\boldsymbol{r})| \Psi_{n}\right\rangle= & \left\langle\psi_{1}\left(\boldsymbol{r}_{1}\right) \ldots \psi_{l}\left(\boldsymbol{r}_{i}\right) \ldots \psi_{\frac{N}{2}}\left(\boldsymbol{r}_{\frac{N}{2}}\right)\left|\sum_{j=1}^{N} \delta\left(\boldsymbol{r}-\boldsymbol{r}_{j}\right)\right| \psi_{1}\left(\boldsymbol{r}_{1}\right) \ldots \psi_{k}\left(\boldsymbol{r}_{i}\right) \ldots \psi_{\frac{N}{2}}\left(\boldsymbol{r}_{\frac{N}{2}}\right)\right\rangle \\
= & \sum_{j=1}^{N} \int d^{3} r_{1} \ldots \int d^{3} r_{i} \ldots \int d^{3} r_{\frac{N}{2}} \psi_{1}^{*}\left(\boldsymbol{r}_{1}\right) \ldots \psi_{l}^{*}\left(\boldsymbol{r}_{i}\right) \ldots \psi_{\frac{N}{2}}^{*}\left(\boldsymbol{r}_{\frac{N}{2}}\right) \delta\left(\boldsymbol{r}-\boldsymbol{r}_{j}\right) \\
& \psi_{1}\left(\boldsymbol{r}_{1}\right) \ldots \psi_{k}\left(\boldsymbol{r}_{i}\right) \ldots \psi_{\frac{N}{2}}\left(\boldsymbol{r}_{\frac{N}{2}}\right) .
\end{aligned}
$$

Abrindo cada termo da somatória, a função delta irá atuar apenas sobre a $j$-ésima coordenada eletrônica,

\footnotetext{
${ }^{2}$ Algumas propostas envolvendo mais de uma excitação foram introduzidas [116] porém grande parte dos códigos comerciais (como os utilizados ao longo deste projeto) possuem apenas excitações simples implementadas.
} 


$$
\begin{aligned}
\left\langle\Psi_{0}|\hat{\rho}(\boldsymbol{r})| \Psi_{n}\right\rangle= & \int d^{3} r_{2} \ldots \int d^{3} r_{i} \ldots \int d^{3} r_{\frac{N}{2}} \overbrace{\left[\int d^{3} r_{1} \psi_{i}^{*}\left(\boldsymbol{r}_{1}\right) \delta\left(\boldsymbol{r}-\boldsymbol{r}_{1}\right) \psi_{1}\left(\boldsymbol{r}_{1}\right)\right]}^{\psi_{1}^{*}(\boldsymbol{r}) \psi_{1}(\boldsymbol{r})} \psi_{2}^{*}\left(\boldsymbol{r}_{2}\right) \ldots \psi_{l}^{*}\left(\boldsymbol{r}_{i}\right) \ldots \\
& \ldots \psi_{\frac{N}{2}}^{*}\left(\boldsymbol{r}_{\frac{N}{2}}\right) \psi_{2}\left(\boldsymbol{r}_{2}\right) \ldots \psi_{k}\left(\boldsymbol{r}_{i}\right) \ldots \psi_{\frac{N}{2}}\left(\boldsymbol{r}_{\frac{N}{2}}\right)+\ldots \\
= & \psi_{1}^{*}(\boldsymbol{r}) \psi_{1}(\boldsymbol{r}) \underbrace{\int d^{3} r_{2} \psi_{2}^{*}\left(\boldsymbol{r}_{2}\right) \psi_{2}\left(\boldsymbol{r}_{2}\right)}_{1} \ldots \underbrace{\int d^{3} r_{i} \psi_{l}^{*}\left(\boldsymbol{r}_{i}\right) \psi_{k}\left(\boldsymbol{r}_{i}\right)}_{\delta_{l k}} \ldots \underbrace{\int d^{3} r_{\frac{N}{2}} \psi_{\frac{N}{2}}^{*}\left(\boldsymbol{r}_{\frac{N}{2}}\right) \psi_{\frac{N}{2}}\left(\boldsymbol{r}_{\frac{N}{2}}\right)}_{1}+\ldots
\end{aligned}
$$

Como os orbitais $\psi_{k}$ e $\psi_{l}$ são diferentes por construção, o único termo da somatória que não é nulo, é aquele cuja delta atua sobre as coordenadas do $i$-ésimo elétron, $\delta\left(\boldsymbol{r}-\boldsymbol{r}_{i}\right)$. Ou seja,

$$
\begin{aligned}
\left\langle\Psi_{0}|\hat{\rho}(\boldsymbol{r})| \Psi_{n}\right\rangle & =\int d^{3} r_{i} \psi_{l}^{*}\left(\boldsymbol{r}_{i}\right) \delta\left(\boldsymbol{r}-\boldsymbol{r}_{i}\right) \psi_{k}\left(\boldsymbol{r}_{i}\right) \overbrace{\int d^{3} r_{1} d^{3} r_{2} \ldots \psi_{1}^{*}\left(\boldsymbol{r}_{1}\right) \ldots \psi_{\frac{N}{2}}\left(\boldsymbol{r}_{\frac{N}{2}}\right)}^{1} \\
& =\psi_{l}^{*}(\boldsymbol{r}) \psi_{k}(\boldsymbol{r}) .
\end{aligned}
$$

De maneira análoga, os demais elementos de matriz são dados por,

$$
\begin{aligned}
& \left\langle\Psi_{n}\left|\hat{\rho}\left(\boldsymbol{r}^{\prime}\right)\right| \Psi_{0}\right\rangle=\psi_{k}^{*}\left(\boldsymbol{r}^{\prime}\right) \psi_{l}\left(\boldsymbol{r}^{\prime}\right), \\
& \left\langle\Psi_{0}\left|\hat{\rho}\left(\boldsymbol{r}^{\prime}\right)\right| \Psi_{n}\right\rangle=\psi_{l}^{*}\left(\boldsymbol{r}^{\prime}\right) \psi_{k}\left(\boldsymbol{r}^{\prime}\right), \\
& \left\langle\Psi_{n}|\hat{\rho}(\boldsymbol{r})| \Psi_{0}\right\rangle=\psi_{k}^{*}(\boldsymbol{r}) \psi_{l}(\boldsymbol{r}) .
\end{aligned}
$$

Tendo em vista que a diferença de energia entre os estados globais $\Psi_{k}$ e $\Psi_{0}$ é simplesmente a diferença entre os autovalores de energia dos orbitais $\psi_{l}$ e $\psi_{k}$,

$$
\Omega_{k} \equiv E_{k}-E_{0}=\epsilon_{k}-\epsilon_{l} \equiv \omega_{l k},
$$

onde $\epsilon_{j}$ é a energia do $j$-ésimo orbital de KS, a função de resposta linear pode ser expressa como,

$$
\chi_{s}\left(\boldsymbol{r}, \boldsymbol{r}^{\prime}, \omega\right)=\lim _{\eta \rightarrow 0+} \sum_{k, l}\left\{\frac{\psi_{l}^{*}(\boldsymbol{r}) \psi_{k}(\boldsymbol{r}) \psi_{k}^{*}\left(\boldsymbol{r}^{\prime}\right) \psi_{l}\left(\boldsymbol{r}^{\prime}\right)}{\omega-\omega_{l k}+i \eta}-\frac{\psi_{l}^{*}\left(\boldsymbol{r}^{\prime}\right) \psi_{k}\left(\boldsymbol{r}^{\prime}\right) \psi_{k}^{*}(\boldsymbol{r}) \psi_{l}(\boldsymbol{r})}{\omega+\omega_{l k}+i \eta}\right\}
$$

A somatória em $k$ atua sobre todos os orbitais desocupados enquanto a em $l$ sobre os ocupados. Para facilitar o cálculo é conveniente introduzir 
o número de ocupação de cada orbital, representado pela letra $f$. Para o $j$-ésimo orbital, $f_{j}=1$ se o orbital está ocupado e $f_{j}=0$ caso contrário. Separando a soma em duas parcelas, mas que agora são sobre todos os orbitais, segue que,

$$
\begin{aligned}
\chi_{s}\left(\boldsymbol{r}, \boldsymbol{r}^{\prime}, \omega\right)= & \lim _{\eta \rightarrow 0+} \sum_{k, l} f_{l}\left(1-f_{k}\right) \frac{\psi_{l}^{*}(\boldsymbol{r}) \psi_{k}(\boldsymbol{r}) \psi_{k}^{*}\left(\boldsymbol{r}^{\prime}\right) \psi_{l}\left(\boldsymbol{r}^{\prime}\right)}{\omega-\omega_{l k}+i \eta} \\
& -\lim _{\eta \rightarrow 0+} \sum_{k, l} f_{l}\left(1-f_{k}\right) \frac{\psi_{l}^{*}\left(\boldsymbol{r}^{\prime}\right) \psi_{k}\left(\boldsymbol{r}^{\prime}\right) \psi_{k}^{*}(\boldsymbol{r}) \psi_{l}(\boldsymbol{r})}{\omega+\omega_{l k}+i \eta} .
\end{aligned}
$$

O termo $f_{l}$ garante que a soma seja realizada quando o l-ésimo orbital é ocupado, enquanto $1-f_{k}$ leva os ocupados a zero, restando apenas os desocupados. Como as somatórias são sobre todos os orbitais, os índices passam a ser mudos, e portanto podemos permutar $l \leftrightarrow k$ na primeira soma, de tal forma que os termos cruzados se anulam, resultando na expressão (2.43),

$$
\begin{aligned}
\chi_{s}\left(\boldsymbol{r}, \boldsymbol{r}^{\prime}, \omega\right)= & \lim _{\eta \rightarrow 0+} \sum_{k, l} f_{k}\left(1-f_{l}\right) \frac{\psi_{k}^{*}(\boldsymbol{r}) \psi_{l}(\boldsymbol{r}) \psi_{l}^{*}\left(\boldsymbol{r}^{\prime}\right) \psi_{k}\left(\boldsymbol{r}^{\prime}\right)}{\omega-\omega_{l k}+i \eta} \\
& -\lim _{\eta \rightarrow 0+} \sum_{k, l} f_{l}\left(1-f_{k}\right) \frac{\psi_{l}^{*}\left(\boldsymbol{r}^{\prime}\right) \psi_{k}\left(\boldsymbol{r}^{\prime}\right) \psi_{k}^{*}(\boldsymbol{r}) \psi_{l}(\boldsymbol{r})}{\omega+\omega_{l k}+i \eta} \\
= & \lim _{\eta \rightarrow 0+} \sum_{k, l}\left(f_{k}-f_{l}\right) \frac{\psi_{l}(\boldsymbol{r}) \psi_{k}^{*}(\boldsymbol{r}) \psi_{k}\left(\boldsymbol{r}^{\prime}\right) \psi_{l}^{*}\left(\boldsymbol{r}^{\prime}\right)}{\omega-\omega_{l k}+i \eta}
\end{aligned}
$$




\section{Apêndice $\mathbf{H}$}

\section{Derivação das equações de Casida}

Para chegar na equação matricial (2.46) partimos da expressão (2.44) que diz respeito a correção de primeira ordem na densidade eletrônica levando em conta efeitos de spin. Na ausência da perturbação externa $v_{1 \sigma}$, a densidade é dada por,

$$
\rho_{1 \sigma}(\boldsymbol{r}, \Omega)=\sum_{\sigma \sigma^{\prime}} \int d^{3} r^{\prime} \chi_{s, \sigma \sigma^{\prime}}\left(\boldsymbol{r}, \boldsymbol{r}^{\prime}, \Omega\right) \int d^{3} r^{\prime \prime} f_{H x c, \sigma \sigma^{\prime}}\left(\boldsymbol{r}^{\prime}, \boldsymbol{r}^{\prime \prime}, \Omega\right) \rho_{1 \sigma^{\prime \prime}}\left(\boldsymbol{r}^{\prime \prime}, \Omega\right) \text {. }
$$

Por simplicidade, definimos o kernel de Hartree-xc como,

$$
f_{H x c, \sigma^{\prime} \sigma^{\prime \prime}}\left(\boldsymbol{r}, \boldsymbol{r}^{\prime}, \omega\right) \equiv \frac{1}{\left\|\boldsymbol{r}-\boldsymbol{r}^{\prime}\right\|}+f_{x c, \sigma^{\prime} \sigma^{\prime \prime}}\left(\boldsymbol{r}, \boldsymbol{r}^{\prime}, \omega\right) .
$$

Como o resultado é geral para qualquer frequência $\omega$, em particular podemos fixar $\omega=\Omega$ sendo $\Omega$ uma energia de excitação. Introduzindo a notação auxiliar,

$$
g_{\sigma \sigma^{\prime}}(\boldsymbol{r}, \Omega) \equiv \int d^{3} r^{\prime} f_{H x c, \sigma \sigma^{\prime}}\left(\boldsymbol{r}, \boldsymbol{r}^{\prime}, \Omega\right) \rho_{1 \sigma^{\prime}}\left(\boldsymbol{r}^{\prime}, \Omega\right),
$$

multiplicando a densidade (H.1) por $f_{H x c, \alpha \sigma}(\boldsymbol{x}, \boldsymbol{r}, \Omega)$ e integrando na variável $\boldsymbol{r}$, segue que,

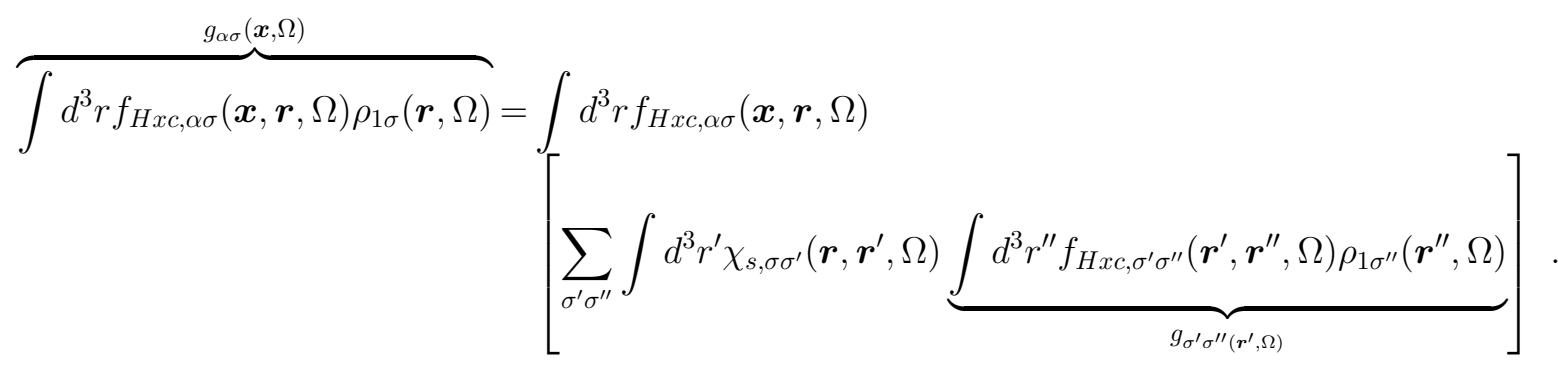

Uma vez que a função de resposta linear do sistema não interagente é descrita pela equação (2.45), com a incorporação dos termos,

$$
\begin{aligned}
\Phi_{l k \sigma}(\boldsymbol{r}) & =\psi_{k, \sigma}^{*}(\boldsymbol{r}) \psi_{l, \sigma}(\boldsymbol{r}) \\
\omega_{l k \sigma} & \equiv \omega_{l k, \sigma}=\epsilon_{k, \sigma}-\epsilon_{l, \sigma} \\
\alpha_{l k \sigma} & =f_{k, \sigma}-f_{l, \sigma}
\end{aligned}
$$


a equação acima passa a ser,

$g_{\alpha \sigma}(\boldsymbol{x}, \Omega)=\int d^{3} r f_{H x c, \alpha \sigma}(\boldsymbol{x}, \boldsymbol{r}, \Omega)\left[\sum_{\sigma^{\prime} \sigma^{\prime \prime}} \int d^{3} r^{\prime} \delta_{\sigma \sigma^{\prime}} \sum_{l, k=1}^{\infty} \alpha_{l k \sigma} \frac{\Phi_{l k \sigma}^{*}(\boldsymbol{r}) \Phi_{l k \sigma}\left(\boldsymbol{r}^{\prime}\right)}{\Omega-\omega_{l k \sigma}+i \eta} g_{\sigma^{\prime} \sigma^{\prime \prime}}\left(\boldsymbol{r}^{\prime}, \Omega\right)\right]$.

A delta de Kronecker elimina uma das somatórias nas coordenadas de spin, de tal forma que,

$g_{\alpha \sigma}(\boldsymbol{x}, \Omega)=\sum_{l, k} \frac{\alpha_{l k \sigma}}{\Omega-\omega_{l k \sigma}+i \eta} \int d^{3} r f_{H x c, \alpha \sigma}(\boldsymbol{x}, \boldsymbol{r}, \Omega) \Phi_{l k \sigma}^{*}(\boldsymbol{r}) \sum_{\sigma^{\prime \prime}} \int d^{3} r^{\prime} \Phi_{l k \sigma} g_{\sigma \sigma^{\prime \prime}}\left(\boldsymbol{r}^{\prime}, \Omega\right)$.

Como a priori as energias de excitação da molécula são distintas das excitações entre orbitais de Kohn-Sham, podemos abandonar o termo i.$^{1}$ Definindo a função auxiliar,

$$
H_{l k \sigma}(\Omega) \equiv \sum_{\sigma^{\prime \prime}} \int d^{3} r \Phi_{l k \sigma}(\boldsymbol{r}) g_{\sigma \sigma^{\prime \prime}}(\boldsymbol{r}, \Omega)
$$

e multiplicando a equação anterior por $\Phi_{l^{\prime} k^{\prime} \alpha}(\boldsymbol{x})$, ao integrar em $\boldsymbol{x}$ e somar em $\sigma$, obtemos que,

$$
\begin{aligned}
\overbrace{\sum_{\sigma} \int d^{3} x \Phi_{l^{\prime} k^{\prime} \alpha}(\boldsymbol{x}) g_{\alpha \sigma}(\boldsymbol{x}, \Omega)}^{H_{l^{\prime} k^{\prime} \alpha}(\Omega)} & =\sum_{\sigma} \int d^{3} x \Phi_{l^{\prime} k^{\prime} \alpha}(\boldsymbol{x})\left[\sum_{l, k} \frac{\alpha_{l k \sigma}}{\Omega-\omega_{l k \sigma}}\right. \\
& \int d^{3} r f_{H x c, \alpha \sigma}(\boldsymbol{x}, \boldsymbol{r}, \Omega) \Phi_{l k \sigma}^{*}(\boldsymbol{r}) \underbrace{\sum_{\sigma^{\prime \prime}} \int d^{3} r^{\prime} \Phi_{l k \sigma}\left(\boldsymbol{r}^{\prime}\right) g_{\sigma \sigma^{\prime \prime}}(\boldsymbol{r}, \Omega)}_{H_{l k \sigma}(\Omega)}] .
\end{aligned}
$$

Rearranjando os termos e definindo a matriz de acoplamento cujos elementos foram introduzidos pela equação (2.49), é possível compactar as equações acima,

$$
H_{l^{\prime} k^{\prime} \alpha}(\Omega)=\sum_{\sigma} \sum_{l, k} \frac{\alpha_{l k \sigma}}{\Omega-\omega_{l k \sigma}} K_{l^{\prime} k^{\prime} \alpha, l k \sigma}(\Omega) H_{l k \sigma}(\Omega)
$$

Trocando as variáveis $l^{\prime} \leftrightarrow l, k^{\prime} \leftrightarrow k, \alpha \rightarrow \sigma, \sigma^{\prime} \rightarrow \sigma$ e definindo,

$$
\beta_{l k \sigma}(\Omega) \equiv \frac{H_{l k \sigma}(\Omega)}{\Omega-\omega_{l k \sigma}}
$$

ao multiplicar e dividir a esquerda por $\Omega-\omega_{l k \sigma}$, segue que,

\footnotetext{
${ }^{1}$ Do ponto de vista da teoria de perturbação introduzida por Görling [117], a energia de excitação de Kohn-Sham corresponde ao termo de ordem zero na expansão do potencial externo em séries de Taylor, e portanto difere da energia de excitação $\Omega$.
} 


$$
\begin{aligned}
\left(\Omega-\omega_{l k \sigma}\right) \beta_{l k \sigma}(\Omega) & =\sum_{\sigma^{\prime}} \sum_{l^{\prime} k^{\prime}} \alpha_{l^{\prime} k^{\prime} \sigma^{\prime}} K_{l k \sigma, l^{\prime} k^{\prime} \sigma^{\prime}}(\Omega) \beta_{l^{\prime} k^{\prime} \sigma^{\prime}}(\Omega) \\
\Omega \beta_{l k \sigma}(\Omega) & =\omega_{l k \sigma} \beta_{l k \sigma}(\Omega)+\sum_{\sigma^{\prime}} \sum_{l^{\prime} k^{\prime}} \alpha_{l^{\prime} k^{\prime} \sigma^{\prime}} K_{l k \sigma, l^{\prime} k^{\prime} \sigma^{\prime}}(\Omega) \beta_{l^{\prime} k^{\prime} \sigma^{\prime}}(\Omega) \\
\Omega \beta_{l k \sigma}(\Omega) & =\sum_{\sigma^{\prime}} \sum_{l^{\prime} k^{\prime}}\left[\alpha_{l^{\prime} k^{\prime} \sigma^{\prime}} K_{l k \sigma, l^{\prime} k^{\prime} \sigma^{\prime}}(\Omega)+\delta_{l l^{\prime}} \delta_{k k^{\prime}} \delta_{\sigma \sigma^{\prime}} \omega_{l^{\prime} k^{\prime} \sigma^{\prime}}\right] \beta_{l^{\prime} k^{\prime} \sigma^{\prime}}(\Omega) .
\end{aligned}
$$

Como $\alpha_{l^{\prime} k^{\prime} \sigma^{\prime}}=f_{k^{\prime}, \sigma^{\prime}}-f_{l^{\prime}, \sigma^{\prime}}$, as únicas transições permitidas são aquelas entre estados com números de ocupação distintos, ou seja, de um estado ocupado para um desocupado e vice-versa. Sejam $i, i^{\prime}$ os índices para os estados ocupados e $a, a^{\prime}$ para desocupados, podemos separar a equação acima em duas equações acopladas,

$$
\begin{gathered}
\sum_{\sigma^{\prime}} \sum_{l^{\prime} k^{\prime}}\left[\delta_{i l^{\prime}} \delta_{a k^{\prime}} \delta_{\sigma \sigma^{\prime}} \omega_{l^{\prime} k^{\prime} \sigma^{\prime}}+\alpha_{l^{\prime} k^{\prime} \sigma^{\prime}} K_{i a \sigma, l^{\prime} k^{\prime} \sigma^{\prime}}(\Omega)\right] \beta_{l^{\prime} k^{\prime} \sigma^{\prime}}(\Omega)=\Omega \beta_{i a \sigma}(\Omega), \\
\sum_{\sigma^{\prime}} \sum_{l^{\prime} k^{\prime}}\left[\delta_{a l^{\prime}} \delta_{i k^{\prime}} \delta_{\sigma \sigma^{\prime}} \omega_{l^{\prime} k^{\prime} \sigma^{\prime}}+\alpha_{l^{\prime} k^{\prime} \sigma^{\prime}} K_{a i \sigma, l^{\prime} k^{\prime} \sigma^{\prime}}(\Omega)\right] \beta_{l^{\prime} k^{\prime} \sigma^{\prime}}(\Omega)=\Omega \beta_{a i \sigma}(\Omega) .
\end{gathered}
$$

Nesta notação, a distribuição $\alpha$ pode ser escrita como,

$$
\alpha_{l^{\prime} k^{\prime} \sigma^{\prime}}=\left\{\begin{array}{rl}
1, & \text { se } k^{\prime}=i^{\prime} \text { e } l^{\prime}=a^{\prime} \\
-1, & \text { se } k^{\prime}=a^{\prime} \text { e } l^{\prime}=i^{\prime} \\
0, & \text { caso contrário }
\end{array},\right.
$$

de tal forma que os elementos das somatórias podem ser separados em duas parcelas, possibilitando que a soma seja realizada sobre os estados ocupados e desocupados,

$$
\begin{aligned}
& \sum_{\sigma^{\prime}} \sum_{i^{\prime} a^{\prime}}\left[\left\{\delta_{i i^{\prime}} \delta_{a a^{\prime}} \delta_{\sigma \sigma^{\prime}} \omega_{i^{\prime} a^{\prime} \sigma^{\prime}}+\alpha_{i^{\prime} a^{\prime} \sigma^{\prime}} K_{i a \sigma, i^{\prime} a^{\prime} \sigma^{\prime}}(\Omega)\right\} \beta_{i^{\prime} a^{\prime} \sigma^{\prime}}+\right. \\
& \left.+\left\{\alpha_{i a^{\prime}} \delta_{a i^{\prime}} \delta_{\sigma \sigma^{\prime}} \omega_{a^{\prime} i^{\prime} \sigma^{\prime}}+\alpha_{a^{\prime} i^{\prime} \sigma^{\prime}} K_{i a \sigma, i^{\prime} a^{\prime} \sigma^{\prime}}(\Omega)\right\} \beta_{a^{\prime} i^{\prime} \sigma^{\prime}}(\Omega)\right]=\Omega \beta_{i a \sigma}(\Omega), \\
& \sum_{\sigma^{\prime}} \sum_{i^{\prime} a^{\prime}}\left[\left\{\delta_{a i^{\prime}} \delta_{i a^{\prime}} \delta_{\sigma \sigma^{\prime}} \omega_{i^{\prime} a^{\prime} \sigma^{\prime}}+\alpha_{i^{\prime} a^{\prime} \sigma^{\prime}} K_{a i \sigma, i^{\prime} a^{\prime} \sigma^{\prime}}(\Omega)\right\} \beta_{i^{\prime} a^{\prime} \sigma^{\prime}}+\right. \\
& \left.+\left\{\alpha_{a a^{\prime}} \delta_{i i^{\prime}} \delta_{\sigma \sigma^{\prime}} \omega_{a^{\prime} i^{\prime} \sigma^{\prime}}+\alpha_{a^{\prime} i^{\prime} \sigma^{\prime}} K_{a i \sigma, a^{\prime} i^{\prime} \sigma^{\prime}}(\Omega)\right\} \beta_{a^{\prime} i^{\prime} \sigma^{\prime}(\Omega)}\right]=\Omega \beta_{i a \sigma}(\Omega) .
\end{aligned}
$$

Devido aos números de ocupação distintos entre estados $i, i^{\prime}$ e $a, a^{\prime}$, as 
funções deltas mistas são nulas, $\delta_{i a^{\prime}}=\delta_{a i^{\prime}}=0$. Substituindo (H.5) sobra que,

$$
\begin{aligned}
& \sum_{\sigma^{\prime}} \sum_{i^{\prime}, a^{\prime}}\left[\left\{\delta_{i i^{\prime}} \delta_{a a^{\prime}} \delta_{\sigma \sigma^{\prime}} \omega_{i^{\prime} a^{\prime} \sigma^{\prime}}-K_{i a \sigma, i^{\prime} a^{\prime} \sigma^{\prime}}(\Omega)\right\} \beta_{i^{\prime} a^{\prime} \sigma^{\prime}}(\Omega)+K_{i a \sigma, a^{\prime} i^{\prime} \sigma^{\prime}}(\Omega) \beta_{a^{\prime} i^{\prime} \sigma^{\prime}}(\Omega)\right]=\Omega \beta_{i a \sigma}(\Omega), \\
& \sum_{\sigma^{\prime}} \sum_{i^{\prime}, a^{\prime}}\left[-K_{a i \sigma, i^{\prime} a^{\prime} \sigma^{\prime}}(\Omega) \beta_{i^{\prime} a^{\prime} \sigma^{\prime}}(\Omega)+\left\{\delta_{i i^{\prime}} \delta_{a a^{\prime}} \delta_{\sigma \sigma^{\prime}} \omega_{a^{\prime} i^{\prime} \sigma^{\prime}}-K_{i a \sigma, a^{\prime} i^{\prime} \sigma^{\prime}}(\Omega)\right\} \beta_{a^{\prime} i^{\prime} \sigma^{\prime}}(\Omega)\right]=\Omega \beta_{i a \sigma}(\Omega) .
\end{aligned}
$$

Como $\omega_{a^{\prime} i^{\prime} \sigma^{\prime}}>0$ pois $a^{\prime}$ é o estado desocupado e $i^{\prime}$ o ocupado, $\omega_{i^{\prime} a^{\prime} \sigma^{\prime}}=$ $-\omega_{a^{\prime} i^{\prime} \sigma^{\prime}}<0$. Definindo $X_{i a \sigma} \equiv-\beta_{i a \sigma}$ e $Y_{i a \sigma} \equiv \beta_{a i \sigma}$, as equações assumem a forma,

$$
\begin{gathered}
\sum_{i^{\prime} a^{\prime} \sigma^{\prime}}\left[\left\{\delta_{i i^{\prime}} \delta_{a a^{\prime}} \delta_{\sigma \sigma^{\prime}} \omega_{a^{\prime} i^{\prime} \sigma^{\prime}}-K_{i a \sigma, i a^{\prime} \sigma^{\prime}}(\Omega)\right\} X_{i^{\prime} a^{\prime} \sigma^{\prime}}+K_{i a \sigma, a^{\prime} i^{\prime} \sigma^{\prime}} Y_{i^{\prime} a^{\prime} \sigma^{\prime}}\right]=-\Omega X_{i a \sigma}, \\
\sum_{i^{\prime} a^{\prime} \sigma^{\prime}}\left[K_{a i \sigma, i^{\prime} a^{\prime} \sigma^{\prime}} X_{i^{\prime} a^{\prime} \sigma^{\prime}}+\left\{\delta_{a a^{\prime}} \delta_{i i^{\prime}} \delta_{\sigma \sigma^{\prime}} \omega_{a^{\prime} i^{\prime} \sigma^{\prime}}+K_{a i \sigma, a^{\prime} i^{\prime} \sigma^{\prime}}(\Omega)\right\} Y_{i^{\prime} a^{\prime} \sigma^{\prime}}\right]=\Omega Y_{i a \sigma} .
\end{gathered}
$$

Assumindo que os orbitais de de KS são reais, o que é permitido se a hamiltoniana do estado fundamental $\mathcal{H}_{0}$ for simétrica frente a inversão temporal, ${ }^{2}$ as equações acima podem ser escritas na forma matricial,

$$
\left(\begin{array}{ll}
\mathbb{A} & \mathbb{B} \\
\mathbb{B} & \mathbb{A}
\end{array}\right)\left(\begin{array}{l}
\boldsymbol{X} \\
\boldsymbol{Y}
\end{array}\right)=\Omega\left(\begin{array}{cc}
-\mathbb{1} & 0 \\
0 & \mathbb{1}
\end{array}\right)\left(\begin{array}{l}
\boldsymbol{X} \\
\boldsymbol{Y}
\end{array}\right),
$$

onde introduzimos os elementos de matriz de $\mathbb{A}$ e $\mathbb{B}$, dados por,

$$
\begin{gathered}
A_{i a \sigma, i^{\prime} a^{\prime} \sigma^{\prime}}(\Omega)=\delta_{i i^{\prime}} \delta_{a a^{\prime}} \delta_{\sigma \sigma^{\prime}} \omega_{a^{\prime} i^{\prime} \sigma^{\prime}}+K_{i a \sigma, i^{\prime} a^{\prime} \sigma^{\prime}}(\Omega), \\
B_{i a \sigma, i a^{\prime} \sigma^{\prime}}(\Omega)=K_{i a \sigma, i^{\prime} a^{\prime} \sigma^{\prime}}(\Omega) .
\end{gathered}
$$

Por fim, a obtenção da expressão (2.50) para a densidade eletrônica pode ser obtida comparando a expressão (H.1) com as grandezas auxiliares. Substituindo as expressões (H.3) e (H.2) de $H_{j k \sigma}(\Omega)$ e $g_{\sigma \sigma^{\prime}}(\boldsymbol{r}, \Omega)$, na definição (H.4) de $\beta_{j k \sigma}(\Omega)$,

\footnotetext{
${ }^{2}$ Tendo em vista o teorema CPT, toda teoria quântica de campos local com hamiltoniana hermitiana que for invariante frente a uma transformação de Lorentz garante simetria frente a conjugação de carga, transformação de paridade e inversão temporal, o que cobre o nível de teoria empregado no presente trabalho.
} 


$$
\begin{aligned}
\beta_{l k \sigma}(\Omega) & =\frac{H_{l k \sigma}(\Omega)}{\Omega-\omega_{l k \sigma}} \\
& =\frac{1}{\Omega-\omega_{l k \sigma}} \sum_{\sigma^{\prime}} \int d^{3} r \Phi_{l k \sigma}(\boldsymbol{r}) g_{\sigma \sigma^{\prime}}(\boldsymbol{r}, \Omega) \\
& =\frac{1}{\Omega-\omega_{l k \sigma}} \sum_{\sigma^{\prime}} \int d^{3} r \Phi_{l k \sigma}(\boldsymbol{r}) \int d^{3} r^{\prime} f_{H x c, \sigma \sigma^{\prime}}\left(\boldsymbol{r}, \boldsymbol{r}^{\prime}, \Omega\right) \rho_{1 \sigma^{\prime}}\left(\boldsymbol{r}^{\prime}, \Omega\right)
\end{aligned}
$$

Por outro lado, substituindo (2.45) na expressão (H.1), sabemos que,

$$
\begin{aligned}
\rho_{1 \sigma}(\boldsymbol{r}, \Omega) & =\sum_{\sigma^{\prime} \sigma^{\prime \prime}} \int d^{3} r^{\prime} \delta_{\sigma \sigma^{\prime}} \sum_{l, k} \alpha_{l k \sigma} \frac{\Phi_{l k \sigma}^{*}(\boldsymbol{r}) \Phi_{l k \sigma}(\boldsymbol{r})}{\Omega-\omega_{l k \sigma}} \int d^{3} r^{\prime \prime} f_{H x c, \sigma^{\prime} \sigma^{\prime \prime}}\left(\boldsymbol{r}^{\prime}, \boldsymbol{r}^{\prime \prime}, \Omega\right) \rho_{1 \sigma^{\prime \prime}}\left(\boldsymbol{r}^{\prime \prime}, \Omega\right) \\
& =\sum_{\sigma^{\prime \prime}} \int d^{3} r^{\prime} \sum_{l, k} \alpha_{l k \sigma} \frac{\Phi_{l k \sigma}^{*}(\boldsymbol{r}) \Phi_{l k \sigma}(\boldsymbol{r})}{\Omega-\omega_{l k \sigma}} \int d^{3} r^{\prime \prime} f_{H x c, \sigma^{\prime} \sigma^{\prime \prime}}\left(\boldsymbol{r}^{\prime}, \boldsymbol{r}^{\prime \prime}, \Omega\right) \rho_{1 \sigma^{\prime \prime}}\left(\boldsymbol{r}^{\prime \prime}, \Omega\right)
\end{aligned}
$$

Comparando as equações (H.6) e (H.7) extraímos que,

$$
\rho_{1 \sigma}(\boldsymbol{r}, \Omega)=\sum_{l, k} \alpha_{l k \sigma} \Phi_{l k \sigma}^{*}(\boldsymbol{r}) \beta_{l k \sigma}(\Omega)
$$

Utilizando as definições das componentes dos vetores $\boldsymbol{X}$ e $\boldsymbol{Y}$, a densidade pode ser expressa de acordo com a equação (2.50),

$$
\begin{aligned}
\rho_{1 \sigma}(\boldsymbol{r}, \Omega) & =\sum_{l, k} \alpha_{l k \sigma} \Phi_{l k \sigma}^{*}(\boldsymbol{r}) \beta_{l k \sigma}(\Omega) \\
& =\sum_{i, a}\left[\alpha_{i a \sigma} \Phi_{i a \sigma}^{*}(\boldsymbol{r}) \beta_{i a \sigma}(\Omega)+\alpha_{a i \sigma} \Phi_{a i \sigma}^{*}(\boldsymbol{r}) \beta_{a i \sigma}(\Omega)\right] \\
& =\sum_{i, a}\left[\Phi_{i a \sigma}^{*}(\boldsymbol{r}) X_{i a \sigma}(\Omega)+\Phi_{i a \sigma}(\boldsymbol{r}) Y_{i a \sigma}(\Omega)\right]
\end{aligned}
$$




\section{Apêndice I}

\section{Funcional de Energia no método DFTB3}

Seguindo a cronologia apresentada na Seção 2.4 e adotando a convenção $\int d \boldsymbol{r} \rightarrow \int, \int d \boldsymbol{r}^{\prime} \rightarrow \int^{\prime}, \rho(\boldsymbol{r}) \rightarrow \rho$ e $\rho\left(\boldsymbol{r}^{\prime}\right) \rightarrow \rho^{\prime}$, ao reescrever a densidade que minimiza o funcional em termos da densidade dos átomos livres, a energia assume a forma,

$$
\begin{aligned}
E\left[\rho_{0}+\Delta \rho\right]= & \sum_{i} n_{i}\left\langle\psi_{i}\left|\left(-\frac{\nabla^{2}}{2}+v(\boldsymbol{r})+\int^{\prime} \frac{\rho_{0}+\Delta \rho^{\prime}}{\left\|\boldsymbol{r}-\boldsymbol{r}^{\prime}\right\|}+v_{x c}(\boldsymbol{r})\right)\right| \psi_{i}\right\rangle \\
& -\frac{1}{2} \iint^{\prime} \frac{\left(\rho_{0}+\Delta \rho\right)\left(\rho_{0}^{\prime}+\Delta \rho^{\prime}\right)}{\left\|\boldsymbol{r}-\boldsymbol{r}^{\prime}\right\|}+E_{x c}\left[\rho_{0}+\Delta \rho\right]-\int v_{x c}(\boldsymbol{r})\left(\rho_{0}+\Delta \rho\right)
\end{aligned}
$$

Separando o termo de primeira ordem dentro do elemento de matriz e reorganizando as integrais de maneira conveniente, segue que,

$$
\begin{aligned}
E\left[\rho_{0}+\Delta \rho\right]= & \sum_{i} n_{i}\left\langle\psi_{i}\left|\left(-\frac{\nabla^{2}}{2}+v(\boldsymbol{r})+\int^{\prime} \frac{\rho_{0}}{\left\|\boldsymbol{r}-\boldsymbol{r}^{\prime}\right\|}+v_{x c}(\boldsymbol{r})\right)\right| \psi_{i}\right\rangle \\
& +\sum_{i} n_{i}\left\langle\psi_{i}\left|\left(\int^{\prime} \frac{\Delta \rho^{\prime}}{\left\|\boldsymbol{r}-\boldsymbol{r}^{\prime}\right\|}\right)\right| \psi_{i}\right\rangle-\frac{1}{2} \iint^{\prime} \frac{\rho_{0}^{\prime}\left(\rho_{0}+\Delta \rho\right)}{\left\|\boldsymbol{r}-\boldsymbol{r}^{\prime}\right\|} \\
& -\frac{1}{2} \iint^{\prime} \frac{\Delta \rho^{\prime}\left(\rho_{0}+\Delta \rho\right)}{\left\|\boldsymbol{r}-\boldsymbol{r}^{\prime}\right\|}+E_{x c}\left[\rho_{0}+\Delta \rho\right]-\int v_{x c}(\boldsymbol{r})\left(\rho_{0}+\Delta \rho\right)
\end{aligned}
$$

De acordo com a equação (2.20), os operadores da primeira somatória são hamiltonianas de Kohn-Sham sujeitas a densidade atômica $\rho_{0}$, identificadas como $\hat{H}_{0}$. Manipulando a segunda somatória,

$$
\sum_{i} n_{i}\left\langle\psi_{i}\left|\left(\int^{\prime} \frac{\Delta \rho^{\prime}}{\left\|\boldsymbol{r}-\boldsymbol{r}^{\prime}\right\|}\right)\right| \psi_{i}\right\rangle=\int^{\prime} \frac{\Delta \rho^{\prime}}{\left\|\boldsymbol{r}-\boldsymbol{r}^{\prime}\right\|} \underbrace{\left(\sum_{i} n_{i}\left|\psi_{i}\right|^{2}\right)}_{\rho_{\min }(\boldsymbol{r})}=\iint^{\prime} \frac{\Delta \rho^{\prime}\left(\rho_{0}+\Delta \rho\right)}{\left\|\boldsymbol{r}-\boldsymbol{r}^{\prime}\right\|}
$$

e realizando uma expansão em série de Taylor até terceira ordem para o funcional de correlação e troca, 


$$
\begin{aligned}
E_{x c}\left[\rho_{0}+\Delta \rho\right] \approx & E_{x c}\left[\rho_{0}\right]+\int\left[\frac{\delta E_{x c}[\rho]}{\delta \rho}\right]_{\rho=\rho_{0}} \Delta \rho \\
& +\frac{1}{2} \iint^{\prime}\left[\frac{\delta^{2} E_{x c}[\rho]}{\delta \rho \delta \rho^{\prime}}\right]_{\rho=\rho_{0}} \Delta \rho \Delta \rho^{\prime} \\
& +\frac{1}{6} \iint^{\prime} \int^{\prime \prime}\left[\frac{\delta^{3} E_{x c}[\rho]}{\delta \rho \delta \rho^{\prime} \delta \rho^{\prime \prime}}\right]_{\rho=\rho_{0}} \Delta \rho \Delta \rho^{\prime} \Delta \rho^{\prime \prime},
\end{aligned}
$$

ao retomar no funcional de energia, alguns dos termos são simplificados,

$$
\begin{aligned}
E\left[\rho_{0}+\Delta \rho\right]= & \sum_{i} n_{i}\left\langle\psi_{i}\left|\hat{H}_{0}\right| \psi_{i}\right\rangle+\frac{1}{2} \iint^{\prime} \frac{\Delta \rho^{\prime}\left(\rho_{0}+\Delta \rho\right)}{\left\|\boldsymbol{r}-\boldsymbol{r}^{\prime}\right\|} \\
& -\frac{1}{2} \iint^{\prime} \frac{\rho_{0}^{\prime}\left(\rho_{0}+\Delta \rho\right)}{\left\|\boldsymbol{r}-\boldsymbol{r}^{\prime}\right\|}-\int v_{x c}(\boldsymbol{r})\left(\rho_{0}+\Delta \rho\right)+E_{x c}\left[\rho_{0}\right] \\
& +\int\left[\frac{\delta E_{x c}[\rho]}{\delta \rho}\right] \Delta \rho+\frac{1}{2} \iint^{\prime}\left[\frac{\delta^{2} E_{x c}[\rho]}{\delta \rho \delta \rho^{\prime}}\right]_{\rho=\rho_{0}} \Delta \rho \Delta \rho^{\prime} \\
& +\frac{1}{6} \iint^{\prime} \int^{\prime \prime}\left[\frac{\delta^{3} E_{x c}[\rho]}{\delta \rho \delta \rho^{\prime} \delta \rho^{\prime \prime}}\right]_{\rho=\rho_{0}} \Delta \rho \Delta \rho^{\prime} \Delta \rho^{\prime \prime} .
\end{aligned}
$$

As contribuições de primeira ordem se anulam, uma vez que todas elas são realizadas sobre todo o espaço e portanto podemos permutar as variáveis $r$ e $r^{\prime}$, resultando na equação (2.54),

$$
\begin{aligned}
E\left[\rho_{0}+\Delta \rho\right]= & \sum_{i} n_{i}\left\langle\psi_{i}\left|\hat{H}_{0}\right| \psi_{i}\right\rangle-\frac{1}{2} \iint^{\prime} \frac{\rho_{0} \rho_{0}^{\prime}}{\left\|\boldsymbol{r}-\boldsymbol{r}^{\prime}\right\|}-\int v_{x c}(\boldsymbol{r}) \rho_{0}+E_{x c}\left[\rho_{0}\right] \\
& +\frac{1}{2} \iint^{\prime}\left(\frac{1}{\left\|\boldsymbol{r}-\boldsymbol{r}^{\prime}\right\|}+\left[\frac{\delta^{2} E_{x c}[\rho]}{\delta \rho \delta \rho^{\prime}}\right]_{\rho=\rho_{0}}\right) \Delta \rho \Delta \rho^{\prime} \\
& +\frac{1}{6} \iint^{\prime} \int^{\prime \prime}\left[\frac{\delta^{3} E_{x c}[\rho]}{\delta \rho \delta \rho^{\prime} \delta \rho^{\prime \prime}}\right]_{\rho=\rho_{0}} \Delta \rho \Delta \rho^{\prime} \Delta \rho^{\prime \prime}
\end{aligned}
$$




\section{Apêndice J}

\section{Procedimento auto-consistente do mo- delo de solvatação implícita}

Dentro da aproximação de BO, é possível desacoplar as coordenadas atômicas e nucleares de tal forma que a densidade do solvente pode ser expressa como,

$$
\rho_{M}(\boldsymbol{r})=\rho_{M}^{n}(\boldsymbol{r})+\rho_{M}^{e}(\boldsymbol{r}),
$$

onde $\rho_{M}^{n}$ é a densidade nuclear, $\rho_{M}^{e}$ a eletrônica e $\boldsymbol{r}$ é o conjunto de coordenadas eletrônicas. Nesse regime o termo de interação entre núcleos é constante de tal forma que o potencial interno é dado pela soma do termo de interação elétron-elétron com o de interação elétron-núcleo ${ }^{1}$. O procedimento iterativo é tipicamente realizado em quatro partes.

1. Determinação da cavidade e repartição da superfície que delimita os meios em elementos de área $\Delta S_{K i}$.

2. Obtenção das cargas pontuais $q_{K i}^{00}$ decorrentes da densidade eletrônica no vácuo $\rho_{M}^{0}$.

3. Cálculo das cargas $q_{K i}^{0, n}$ utilizando o campo elétrico resultante de $\rho_{M}^{0}$ e das cargas $q_{K i}^{0, n-1}$.

4. Determinação da energia $E_{1}$ e $\rho_{M}^{1}$ através da adição da contribuição das cargas polarizadas convergidas.

A primeira delas envolve a construção da cavidade usualmente considerando esferas ou elipsoides centrados em cada átomo do soluto. Em seguida a área que delimita a fronteira entre os dois meios é repartida em regiões menores $\Delta S_{K i}$, onde a notação diz respeito a $i$-ésima área referente ao $K$-ésimo centro (ou átomo). Para cada elemento de superfície é determinada a densidade superficial associada aquela região e são realizadas as próximas etapas descritas acima.

\footnotetext{
${ }^{1}$ Aqui vale destacar que os termos cruzados $U_{n e}$ e $U_{e n}$ são iguais, sendo $U_{n e}$ a contribuição decorrente da interação densidade nuclear com potencial eletrônico e vice-versa.
} 


\section{Apêndice K}

\section{Modelo de dispersão de Slater-Kirkwood}

O tratamento da correção de dispersão no formalismo DFTB é dado por meio da adição de $E_{\text {disp }}$ nos funcionais de energia (2.66) e (2.72), onde essa contribuição busca reproduzir interações intermoleculares e para isso mimetiza o decaimento com $R^{-6}$,

$$
E_{\text {disp }}=-\sum_{\alpha \beta} f\left(R_{\alpha \beta}\right) C_{6}^{\alpha \beta}
$$

As somas em $\alpha$ e $\beta$ correm sobre todos os átomos e os coeficientes diatômicos $C_{\alpha \beta}$ são obtidos a partir do modelo de Slater-Kirkwood. Partindo do trabalho original de 1931 [118], os coeficientes atômicos são dados por,

$$
C_{6}^{\alpha}=0.75 \sqrt{N_{\alpha} p_{\alpha}^{3}},
$$

onde $p_{\alpha}$ é a polarizabilidade do átomo $\alpha$ e $N_{\alpha}$ o número efetivo de elétrons. Aplicando a regra de combinação de Slater-Kirkwood, os coeficientes diatômicos assumem a forma,

$$
C_{6}^{\alpha \beta}=\frac{2 C_{6}^{\alpha} C_{6}^{\beta} p_{\alpha} p_{\beta}}{p_{\alpha}^{2} C_{6}^{\alpha}+p_{\beta}^{2} C_{6}^{\beta}} .
$$

Seguindo a proposta de Halgren [119], o número efetivo de elétrons é 0.8 para o hidrogênio e,

$$
N_{\alpha}=1.17+n_{v}^{\alpha},
$$

para ametais maiores que o carbono, onde $n_{v}^{\alpha}$ é o número de elétrons de valência do $\alpha$-ésimo átomo. A função $f(R)$ de amortecimento é da forma,

$$
f(R)=\left[1-e^{-3\left(\frac{R}{R_{0}}\right)^{7}}\right]^{4},
$$

onde $R_{0}$ é $3.8 \AA$ para átomos da primeira linha da tabela periódica e 4.8 A para átomos da segunda. Esses parâmetros foram otimizados de tal forma a reproduzir o comportamento esperado da interação em função de 
$R$, discutido em [120]. A combinação de raios que aparece na equação (K.1) é dada por,

$$
R_{0}^{\alpha \beta}=\frac{\left(R_{0}^{\alpha}\right)^{3}+\left(R_{0}^{\beta}\right)^{3}}{\left(R_{0}^{\alpha}\right)^{2}+\left(R_{0}^{\beta}\right)^{2}} .
$$

A partir das equações acima verificamos que o modelo necessita que sejam fornecidos os valores de polarizabilidade atômica $\left(p_{\alpha}\right)$, dos raios covalentes $\left(R_{0}^{\alpha}\right)$ e número efetivo de elétrons, que é dado em termos da carga $\left(q_{\alpha}\right)$. Como a polarizabilidades e os raios são sensíveis ao ambiente que circunda os átomos, uma forma alternativa consiste em fornecer os valores de acordo com o número de primeiros vizinhos. A Tabela K.1 utiliza do raio de van der Waals como sendo o raio covalente e traz os valores utilizados nos cálculos.

\begin{tabular}{|c|c|c|c|}
\hline Átomo & Polarizabilidade atômica $\left(\AA^{3}\right)$ & Raio de van der Waals $(\AA)$ & Carga $(e)$ \\
\hline $\mathrm{C}$ & 1.7781 .4421 .4421 .4331 .2801 .280 & 3.73 .73 .73 .73 .73 .7 & 2.50 \\
\hline $\mathrm{H}$ & 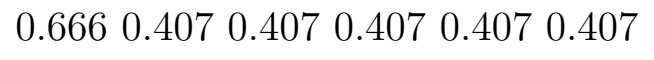 & 3.23 .23 .23 .23 .23 .2 & 0.80 \\
\hline $\mathrm{O}$ & $\begin{array}{lllll}0.800 & 0.729 & 0.713 & 0.713 & 0.7130 .713\end{array}$ & 3.73 .73 .73 .73 .73 .7 & 3.15 \\
\hline $\mathrm{N}$ & 1.0960 .9420 .9420 .9420 .9420 .942 & 3.73 .73 .73 .73 .73 .7 & 2.82 \\
\hline $\mathrm{S}$ & 2.9002 .7002 .7002 .7002 .7002 .700 & $4.24 .2 \quad 4.2 \quad 4.2 \quad 4.2 \quad 4.2$ & 4.80 \\
\hline
\end{tabular}

Tabela K.1: Parâmetros considerados na correção de dispersão do modelo de SlaterKirkwood. As colunas com polarizabilidades atômicas e raios de van der Waals fornecem os valores variando o número de primeiros vizinhos de 0 até 5 ou mais, respectivamente.

Os 6 conjuntos de raios e polarizabilidades correspondem, respectivamente, aos valores quando o número de primeiros vizinhos varia entre $0,1,2,3$, 4 até 5 ou mais. A escolha desses parâmetros parte dos valores padrão implementados no código DFTB + e foi otimizada para as moléculas de tiofeno, benzotiadiazol e benzotriazol de tal forma a convergir as geometrias a nível DFTB com as obtidas via DFT/wB97X-D/6-31G(d,p) [23]. 


\section{Apêndice L}

\section{Comparação entre geometrias}

Como foi discutido na Subseção 3.2.1, nos cálculos DFTB o parâmetro de longo alcance ótimo muda substancialmente quando são levados em consideração os valores experimentais de $I P$ e $E A$. No que diz respeito às geometrias, a Tabela L.1 fornece os resultados do desvio quadrático médio (RMSD), calculado a partir da equação (3.1), tomando como referência a geometria otimizada via DFT, i.e., DFT/wB97X-D/6-31G(d,p).

\begin{tabular}{c|ccc} 
& \multicolumn{3}{|c}{ RMSD $(\AA)$} \\
\hline$\omega\left(a_{0}^{-1}\right)$ & Naftaleno & Antraceno & Pireno \\
\hline 0.1 & 0.0060 & 0.0062 & 0.0054 \\
0.2 & 0.0075 & 0.0099 & 0.0088 \\
0.3 & 0.0097 & 0.0140 & 0.0126 \\
0.4 & 0.0118 & 0.0172 & 0.0161 \\
0.5 & 0.0124 & 0.0185 & 0.0169 \\
\hline Média & 0.0095 & 0.0132 & 0.0119 \\
DAM & 0.0022 & 0.0041 & 0.0039 \\
\hline
\end{tabular}

Tabela L.1: Raízes do desvio quadrático médio (RMSD) dos comprimentos de ligação, obtidos via $D F T B$, em relação a geometria otimizada a nível $\omega \mathrm{B} 97 \mathrm{X}-\mathrm{D} / 6-31 \mathrm{G}(\mathrm{d}, \mathrm{p})$, para as moléculas de naftaleno, antraceno e pireno. As duas últimas linhas dizem respeito a média e o desvio absoluto médio.

Novamente as variações apresentam um comportamento bem definido, aumentando as diferenças conforme aumentamos o parâmetro de longo alcance. Como a molécula de antraceno possui 26 ligações, o desvio para $\omega=0.5 a_{0}^{-1}$ implica na variação total da ordem de $0.48 \AA$, que, por ser diluída ao longo dos átomos, é pequena. Consequentemente, o impacto na simetria dos compostos também é pequeno, nesses casos podendo ser entendido como uma consequência da implementação dos algoritmos de otimização de geometria, que não leva em consideração o grupo pontual de simetria. Inspecionando explicitamente os comprimentos de ligação da molécula de naftaleno, o anel que na representação da Figura 3.4 possui 
três ligações duplas acaba apresentando comprimentos maiores nos cálculos $D F T B$ (em relação ao DFT) para essas ligações e menores para as simples. Já no anel com duas ligações, o comportamento é o oposto, ligações duplas são menores nos cálculos $D F T B$ e as simples ligeiramente maiores. Por outro lado, todas as ligações C-H são maiores para a DFTB e com menor variação entre elas.

Comparando resultados DFTB fica claro que o comportamento destacado acima é intensificado com o aumento de $\omega$. Ligações que eram maiores, com relação à otimização DFT, ficam ainda maiores e vice-versa. Variações nos ângulos de ligação e torsão são desprezíveis e, por se tratar de compostos rígidos, podem ser entendidas como consequência das variações dos comprimentos de ligação. Cargas parciais também não mudam com a escolha de $\omega$. Por completeza, comparações entre geometrias DFTB variando $\omega$ são apresentadas nas tabelas abaixo. A Tabela L.2 calcula o RMSD em relação à geometria otimizada com $\omega=0.3 a_{0}^{-1}$ enquanto a Tabela L.3 em relação à geometria otimizada com $\omega=0.5 a_{0}^{-1}$.

\begin{tabular}{c|ccc} 
& \multicolumn{3}{|c}{ RMSD $(\AA)$} \\
\hline$\omega\left(a_{0}^{-1}\right)$ & Naftaleno & Antraceno & Pireno \\
\hline 0.1 & 0.0069 & 0.0099 & 0.0094 \\
0.2 & 0.0032 & 0.0046 & 0.0044 \\
0.4 & 0.0027 & 0.0036 & 0.0037 \\
0.5 & 0.0033 & 0.0049 & 0.0045 \\
\hline Média & 0.0040 & 0.0057 & 0.0055 \\
DAM & 0.0014 & 0.0021 & 0.0019 \\
\hline
\end{tabular}

Tabela L.2: Raízes do desvio quadrático médio (RMSD) dos comprimentos de ligação, obtidos via $D F T B$, em relação a geometria otimizada com $\omega=0.3 a_{0}^{-1}$, para as moléculas de naftaleno, antraceno e pireno. As duas últimas linhas dizem respeito a média e o desvio absoluto médio. 


\begin{tabular}{c|ccc} 
& \multicolumn{3}{|c}{ RMSD $(\AA)$} \\
\hline$\omega\left(a_{0}^{-1}\right)$ & Naftaleno & Antraceno & Pireno \\
\hline 0.1 & 0.0101 & 0.0147 & 0.0139 \\
0.2 & 0.0065 & 0.0095 & 0.0089 \\
0.3 & 0.0033 & 0.0049 & 0.0045 \\
0.4 & 0.0009 & 0.0016 & 0.0010 \\
\hline Média & 0.0052 & 0.0077 & 0.0071 \\
DAM & 0.0031 & 0.0044 & 0.0043 \\
\hline
\end{tabular}

Tabela L.3: Raízes do desvio quadrático médio (RMSD) dos comprimentos de ligação, obtidos via $D F T B$, em relação a geometria otimizada com $\omega=0.5 a_{0}^{-1}$, para as moléculas de naftaleno, antraceno e pireno. As duas últimas linhas dizem respeito a média e o desvio absoluto médio. 


\section{Apêndice M}

\section{Estados excitados dos HPAs}

A tabela abaixo fornece as energias e respectivas forças de oscilador de algumas excitações dos HPAs. As primeiras cinco excitações buscam elucidar o adensamento de estados não acessíveis via $D F T B$, discutido na Seção 3.3. As transições de maior intensidade ilustram o comportamento dos picos de absorção dos HPAs, generalizando o fenômeno observado na Figura 3.9 do naftaleno para os demais hidrocarbonetos. 


\begin{tabular}{|c|c|c|c|c|c|c|c|c|c|}
\hline \multirow[b]{3}{*}{$\omega\left(a_{0}^{-1}\right)$} & \multicolumn{3}{|c|}{ Naftaleno } & \multicolumn{3}{|c|}{ Antraceno } & \multicolumn{3}{|c|}{ Pireno } \\
\hline & \multirow{2}{*}{$\begin{array}{c}\boldsymbol{D F T} \\
0.2 \\
\end{array}$} & \multicolumn{2}{|c|}{$D F T B$} & \multirow{2}{*}{$\begin{array}{c}\boldsymbol{D F T} \\
0.2 \\
\end{array}$} & \multicolumn{2}{|c|}{$D F T B$} & \multirow{2}{*}{$\begin{array}{c}\boldsymbol{D F T} \\
0.2 \\
\end{array}$} & \multicolumn{2}{|c|}{$D F T B$} \\
\hline & & 0.3 & 0.5 & & 0.3 & 0.5 & & 0.3 & 0.5 \\
\hline$S_{0} \rightarrow S_{1}(\mathrm{eV})$ & 4.736 & 4.995 & 5.273 & 3.715 & 3.867 & 4.138 & 4.070 & 4.131 & 4.400 \\
\hline$f_{1}$ & 0.000 & 0.087 & 0.097 & 0.089 & 0.108 & 0.133 & 0.000 & 0.353 & 0.399 \\
\hline$S_{0} \rightarrow S_{2}(\mathrm{eV})$ & 4.836 & 5.137 & 5.412 & 4.159 & 4.444 & 4.689 & 4.177 & 4.328 & 4.615 \\
\hline$f_{2}$ & 0.078 & 0.043 & 0.056 & 0.003 & 0.135 & 0.166 & 0.322 & 0.030 & 0.035 \\
\hline$S_{0} \rightarrow S_{3}(\mathrm{eV})$ & 6.330 & 5.614 & 5.926 & 5.341 & 4.853 & 5.145 & 4.957 & 4.853 & 5.145 \\
\hline$f_{3}$ & 1.270 & 0.000 & 0.000 & 0.000 & 0.000 & 0.000 & 0.000 & 0.000 & 0.000 \\
\hline$S_{0} \rightarrow S_{4}(\mathrm{eV})$ & 6.580 & 5.927 & 6.229 & 5.594 & 5.235 & 5.528 & 5.157 & 4.943 & 5.310 \\
\hline$f_{4}$ & 0.255 & 0.000 & 0.000 & 2.030 & 0.000 & 0.000 & 0.000 & 0.000 & 0.000 \\
\hline$S_{0} \rightarrow S_{5}(\mathrm{eV})$ & 6.637 & 6.415 & 6.775 & 5.642 & 5.254 & 5.603 & 5.205 & 5.185 & 5.497 \\
\hline$f_{5}$ & 0.000 & 0.000 & 0.000 & 0.000 & 0.000 & 0.000 & 0.423 & 0.000 & 0.000 \\
\hline$S_{0} \rightarrow S_{B 1}(\mathrm{eV})$ & 4.836 & 4.995 & 5.273 & 3.715 & 4.444 & 4.689 & 4.117 & 4.131 & 4.400 \\
\hline$f_{B 1}$ & 0.078 & 0.087 & 0.097 & 0.089 & 0.135 & 0.166 & 0.322 & 0.353 & 0.399 \\
\hline$S_{0} \rightarrow S_{B 2}(\mathrm{eV})$ & 6.330 & 5.137 & 5.412 & 5.594 & 5.952 & 6.260 & 5.205 & 5.431 & 5.844 \\
\hline$f_{B 2}$ & 1.270 & 0.043 & 0.056 & 2.030 & 1.854 & 1.980 & 0.423 & 0.435 & 0.559 \\
\hline$S_{0} \rightarrow S_{B 3}(\mathrm{eV})$ & 6.580 & 6.644 & 6.944 & 6.300 & 6.399 & 6.719 & 5.851 & 5.996 & 6.293 \\
\hline$f_{B 3}$ & 0.255 & 1.222 & 1.315 & 0.139 & 0.154 & 0.213 & 0.998 & 0.883 & 1.037 \\
\hline$S_{0} \rightarrow S_{B 4}(\mathrm{eV})$ & 8.751 & 6.723 & 7.028 & 8.043 & 8.049 & 8.799 & 7.164 & 7.475 & 7.796 \\
\hline$f_{B 4}$ & 0.425 & 0.284 & 0.353 & 0.424 & 0.353 & 0.357 & 0.708 & 0.722 & 0.148 \\
\hline$S_{0} \rightarrow S_{B 5}(\mathrm{eV})$ & 10.710 & 9.105 & 9.674 & 9.641 & 10.036 & 10.921 & 7.796 & 8.092 & 8.670 \\
\hline$f_{B 5}$ & 0.061 & 0.419 & 0.402 & 0.154 & 0.277 & 0.209 & 0.845 & 0.676 & 0.730 \\
\hline
\end{tabular}

Tabela M.1: Energias de transição e respectivas forças de oscilador $(f)$ dos primeiros 5 estados excitados $\left(S_{i}\right)$ e dos primeiros 5 estados oticamente ativos $\left(S_{B i}\right)$ dos compostos naftaleno, antraceno e pireno. Os resultados foram obtidos com os métodos $D F T$ e $D F T B$, utilizando o parâmetro de longo alcance otimizado via $J_{3}\left(\omega=0.2 a_{0}^{-1}\right.$ para $D F T$ e $\omega=0.5 a_{0}^{-1}$ para $\left.D F T B\right)$ e via $J_{3}^{\exp }\left(\omega=0.3 a_{0}^{-1}\right.$ para $\left.D F T B\right)$. 


\section{Apêndice N}

\section{Força de oscilador, seção de choque e polarizabilidade}

A força de oscilador associada a transição do estado inicial $n$ para o estado final $k$ é calculada a partir de,

$$
f_{n k}=\frac{2 m_{e}}{3 \hbar^{2}}\left(E_{k}-E_{n}\right) \sum_{\alpha=x, y, z}\left|\left\langle n\left|\hat{\mathcal{R}}_{\alpha}\right| k\right\rangle\right|^{2},
$$

onde $\hat{\mathcal{R}}_{\alpha}$ é a componente $\alpha$ do operador de posição, dado pela soma dos operadores de posição de cada um dos $N$ elétrons,

$$
\hat{\mathcal{R}}_{\alpha}=\sum_{i}^{N} \boldsymbol{r}_{i, \alpha} .
$$

A origem do conceito remete ao estudo do índice de refração em meios dispersivos e do decaimento do átomo clássico, que foram posteriormente adaptados para um tratamento semi-clássico [121, 122]. Considerando um composto quântico sujeito a uma perturbação externa pequena, em primeira ordem a regra de ouro de Fermi enuncia que a probabilidade de transição por unidade de tempo, $\Gamma_{n \rightarrow k}$, de um estado inicial $|n\rangle$ para um estado final $|k\rangle$ é dada por,

$$
\Gamma_{n \rightarrow k}=\frac{2 \pi}{\hbar}\left|\left\langle k\left|\hat{\mathcal{H}}_{1}\right| n\right\rangle\right|^{2} \rho\left(E_{k}\right),
$$

onde $\hat{\mathcal{H}}_{1}$ é a perturbação externa e $\rho\left(E_{k}\right)$ a densidade de estados com energia $E_{k}$. Quando a perturbação admite expansão multipolar, o operador de posição $\hat{\mathcal{R}}$ é introduzido, estabelecendo a conexão entre probabilidade de transição e força de oscilador. No caso de um fóton retratado como uma onda plana monocromática, a perturbação em primeira ordem acopla o momento linear com o campo elétrico [122] resultando em,

$$
\Gamma_{n \rightarrow k}=\frac{\pi}{3 \hbar^{2}} \sum_{\alpha}\left|\left\langle n\left|\hat{\mathcal{R}}_{\alpha}\right| k\right\rangle\right|^{2}
$$


Como a potência total absorvida é dada tanto pela integral da seção choque $(\sigma)$ quanto pela energia do fóton associada a probabilidade de transição, ambas as grandezas são diretamente proporcionais, e portanto $\sigma \propto \Gamma \propto f$. Durante o processo de espalhamento são observadas estruturas ressonantes cujas soluções das equações diferenciais que as regem são tipicamente lorentzianas, o que leva a representação do espectro de absorção como uma convolução de lorentzianas centradas nas energias das forças de oscilador.

Uma conexão importante pode ser estabelecida entre a força de oscilador e a polarizabilidade eletrônica. Em particular no caso em que a perturbação $\hat{\mathcal{H}}_{1}$ é fruto de um campo constante, o acoplamento via momento de dipolo permanece, com o detalhe de que não há o termo oscilatório dependente da frequência da radiação, $\omega$. Olhando para a componente $\mathcal{E}_{z}$ do campo elétrico, pelo teorema de Hellmann-Feynman, sabemos que,

$$
\frac{\partial E}{\partial \mathcal{E}_{z}}=\left\langle\frac{\partial \hat{\mathcal{H}}}{\partial \mathcal{E}_{z}}\right\rangle=\left\langle\frac{\partial \hat{\mathcal{H}}_{1}}{\partial \mathcal{E}_{z}}\right\rangle=\left\langle\frac{\partial\left(-\mu_{z} \mathcal{E}_{z}\right)}{\partial \mathcal{E}_{z}}\right\rangle=-\left\langle\mu_{z}\right\rangle,
$$

onde $\hat{\mathcal{H}}=\hat{\mathcal{H}}_{0}+\hat{\mathcal{H}}_{1}$ é a hamiltoniana total e $\mu_{z}$ a componente $z$ do momento de dipolo. Tendo em vista a relação acima, ao tomar a expansão em polinômios de Taylor da energia $E$ em torno de $\mathcal{E}_{z}=0$, segue que,

$$
E=E^{(0)}+\left(\frac{\partial \hat{\mathcal{H}}}{\partial \mathcal{E}_{z}}\right)_{\mathcal{E}_{z}=0} \mathcal{E}_{z}+\frac{1}{2 !}\left(\frac{\partial^{2} \hat{\mathcal{H}}}{\partial \mathcal{E}_{z}^{2}}\right)_{\mathcal{E}_{z}=0} \mathcal{E}_{z}^{2}+\frac{1}{3 !}\left(\frac{\partial^{3} \hat{\mathcal{H}}}{\partial \mathcal{E}_{z}^{3}}\right)_{\mathcal{E}_{z}=0} \mathcal{E}_{z}^{3}+\cdots
$$

Derivando ambos os lados com relação a $\mathcal{E}_{z}$, chegamos na expansão de Taylor da componente $z$ do momento de dipolo,

$$
\begin{aligned}
\left\langle\mu_{z}\right\rangle & =-\left(\frac{\partial \hat{\mathcal{H}}}{\partial \mathcal{E}_{z}}\right)_{\mathcal{E}_{z}=0}-\left(\frac{\partial^{2} \hat{\mathcal{H}}}{\partial \mathcal{E}_{z}^{2}}\right)_{\mathcal{E}_{z}=0} \mathcal{E}_{z}-\frac{1}{2}\left(\frac{\partial^{3} \hat{\mathcal{H}}}{\partial \mathcal{E}_{z}^{3}}\right)_{\mathcal{E}_{z}=0} \mathcal{E}_{z}^{2}+\cdots \\
& \equiv \mu_{0 z}+\alpha_{z z} \mathcal{E}_{z}+\beta_{z z z} \mathcal{E}_{z}^{2}+\cdots
\end{aligned}
$$

Por ser dada pelo termo de segunda ordem da expansão da energia, o elemento de matriz $\alpha_{z z}$ da polarizabilidade assume a forma, 


$$
\alpha_{z z}=-2 \sum_{n>0} \frac{\left\langle 0^{(0)}\left|\hat{\mu}_{z}\right| n^{(0)}\right\rangle\left\langle n^{(0)}\left|\hat{\mu}_{z}\right| 0^{(0)}\right\rangle}{E_{0}^{(0)}-E_{n}^{(0)}}=2 \sum_{n>0} \frac{\left(\mu_{z}\right)_{0 n}\left(\mu_{z}\right)_{n 0}}{\Delta E_{n 0}}
$$

sendo $\left(\mu_{z}\right)_{0 n}$ a componente $z$ do momento de dipolo de transição do estado fundamental para o $n$-ésimo estado, $\left|i^{(0)}\right\rangle$ o $i$-ésimo autoestado de $\hat{\mathcal{H}}_{0}$ e $E_{i}^{(0)}$ a respectiva autoenergia. Pensando agora na polarizabilidade média/isotrópica $\left(\alpha_{0}\right)$ e tendo em vista a equação (N.1), a conexão com a força de oscilador fica evidente, ${ }^{1}$

$$
\begin{aligned}
\alpha_{0} & =\frac{\alpha_{x x}+\alpha_{y y}+\alpha_{z z}}{3} \\
& =\frac{2}{3} \sum_{n>0}\left[\frac{\left(\mu_{x}\right)_{0 n}\left(\mu_{x}\right)_{n 0}+\left(\mu_{y}\right)_{0 n}\left(\mu_{y}\right)_{n 0}+\left(\mu_{z}\right)_{0 n}\left(\mu_{z}\right)_{n 0}}{\Delta E_{n 0}}\right] \\
& =\frac{2}{3} \sum_{n>0} \frac{\boldsymbol{\mu}_{0 n} \cdot \boldsymbol{\mu}_{n 0}}{\Delta E_{n 0}}=\frac{2}{3} \sum_{n>0} \frac{\left|\boldsymbol{\mu}_{0 n}\right|^{2}}{\Delta E_{n 0}} \\
& =\frac{2}{3} \sum_{n>0} \frac{1}{\Delta E_{n 0}}\left(e^{2} \sum_{\alpha=x, y, z}\left|\left\langle 0^{(0)}\left|\hat{\mathcal{R}}_{\alpha}\right| n^{(0)}\right\rangle\right|^{2}\right) \\
& =\frac{2}{3} \sum_{n>0} \frac{e^{2}}{\Delta E_{n 0}}\left(\frac{3 \hbar^{2}}{2 m_{e} \Delta E_{n 0}} f_{0 n}\right) \\
& =\frac{\hbar^{2} e^{2}}{m_{e}} \sum_{n>0} \frac{f_{0 n}}{\left(\Delta E_{n 0}\right)^{2}} .
\end{aligned}
$$

\footnotetext{
${ }^{1}$ A definição da força de oscilador não é absoluta, de tal forma que as constantes que relacionam $\alpha_{0}$ e $f_{0 n}$ podem mudar de acordo com o autor.
} 


\section{Apêndice $O$}

\section{Validação do algoritmo de cálculo do volume molecular}

A estimativa dos volumes em nível DFTB foi realizada a partir de esferas centradas nos átomos. Para cada átomo é atribuído o raio de van der Waals correspondente, obtido a partir da parametrização DFT-D3 [110], e através de um algoritmo empregando o método de Monte Carlo [123], i.e., calculando a razão entre os o número de pontos gerados aleatoriamente que se encontram dentro e fora das esferas, determinamos o volume da molécula. A Figura O.1 ilustra em verde a superfície utilizada para estimar o volume do aceitador DTP-IC-4Ph na ausência de cadeias laterais.

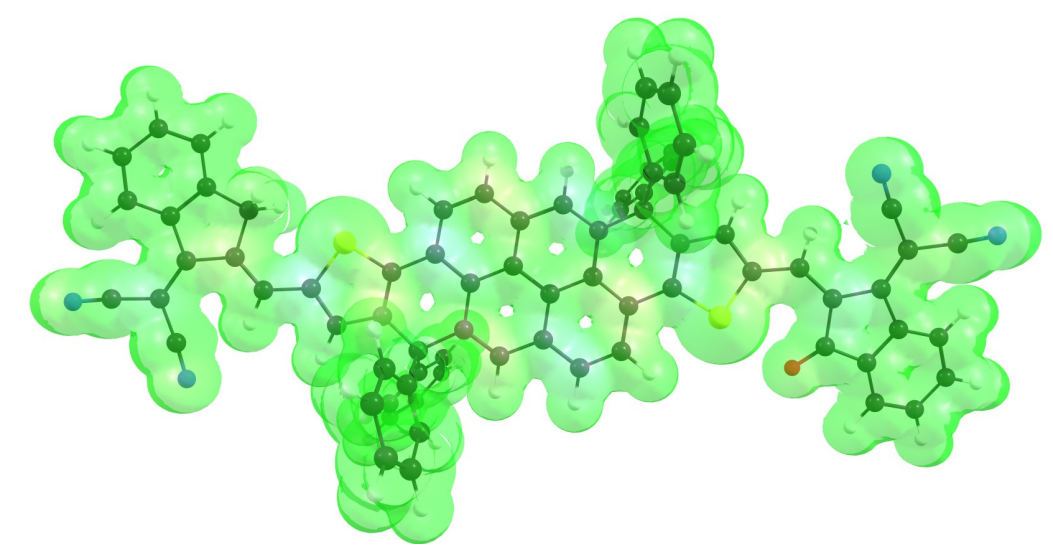

Figura O.1: Superfície delimitada pelas esferas de van-der-Waals cujo interior estima o volume do composto, nesse caso, o aceitador DTP-IC-4Ph sem as cadeias laterais.

A validação do algoritmo foi realizada estudando compostos pequenos e comparando com os resultados obtidos via DFT a partir da densidade eletrônica com o valor de corte de 0.001 elétrons $/$ bohr $^{3}$ [124]. Para a escolha das moléculas, o principal critério foi a representatividade do conjunto, tanto na composição atômica, caracterizando a interação dos 5 átomos (C, H, O, N e S) que constituem o composto DTP-IC-4Ph, quanto da perspectiva estrutural dos anéis aromáticos $\pi$-conjugados. A Tabela O.1 traz os resultados para um conjunto representativo de pequenas moléculas. 


\begin{tabular}{c|c|cc} 
& DFT & \multicolumn{2}{|c}{ DFTB } \\
\hline Sistema & $V\left(\AA^{3}\right)$ & $V\left(\AA^{3}\right)$ & $D P\left(\AA^{3}\right)$ \\
\hline Água $\left(\mathrm{H}_{2} \mathrm{O}\right)$ & 22.85 & 25.90 & 0.11 \\
Amônia $\left(\mathrm{NH}_{3}\right)$ & 35.49 & 32.03 & 0.07 \\
Metano $\left(\mathrm{CH}_{4}\right)$ & 41.00 & 39.25 & 0.19 \\
Etanol $\left(\mathrm{C}_{2} \mathrm{H}_{6} \mathrm{O}\right)$ & 72.26 & 70.62 & 0.29 \\
Benzeno $\left(\mathrm{C}_{6} \mathrm{H}_{6}\right)$ & 107.87 & 107.43 & 0.40 \\
Tiofeno $\left(\mathrm{C}_{4} \mathrm{H}_{4} \mathrm{~S}\right)$ & 93.38 & 100.52 & 0.27 \\
\hline
\end{tabular}

Tabela O.1: Volumes das moléculas de água, amônia, metano, etanol, benzeno e tiofeno, obtidos utilizando ambas as metodologias. A coluna DP diz respeito ao desvio padrão associado à média de 10 cálculos com $10^{6}$ pontos em cada um.

Confrontando os formalismos verificamos que para as moléculas de água e tiofeno a $D F T B$ prediz volumes maiores em relação a $D F T$, enquanto as demais manifestam comportamento oposto. No que diz respeito aos átomos, ao comparar os resultados da água e do etanol, inferimos que o raio de van der Waals do oxigênio tende a superestimar o comprimento de ligação O-H e subestimar o O-C. Examinando o metano e a amônia, inferimos que ambas as ligações $\mathrm{C}-\mathrm{H}$ e N-H são minimizadas. Por outro lado, as estruturas ressonantes do benzeno convergem dentro do intervalo de confiança de $1 \sigma$, o que aponta para a ligação C-S superestimada.

O volume DFTB corresponde a uma média de 10 cálculos realizados com $10^{6}$ pontos gerados aleatoriamente em cada cálculo. A convergência pode ser observada na Figura O.2, que traz os resultados de um dos 10 cálculos da molécula de tiofeno. 

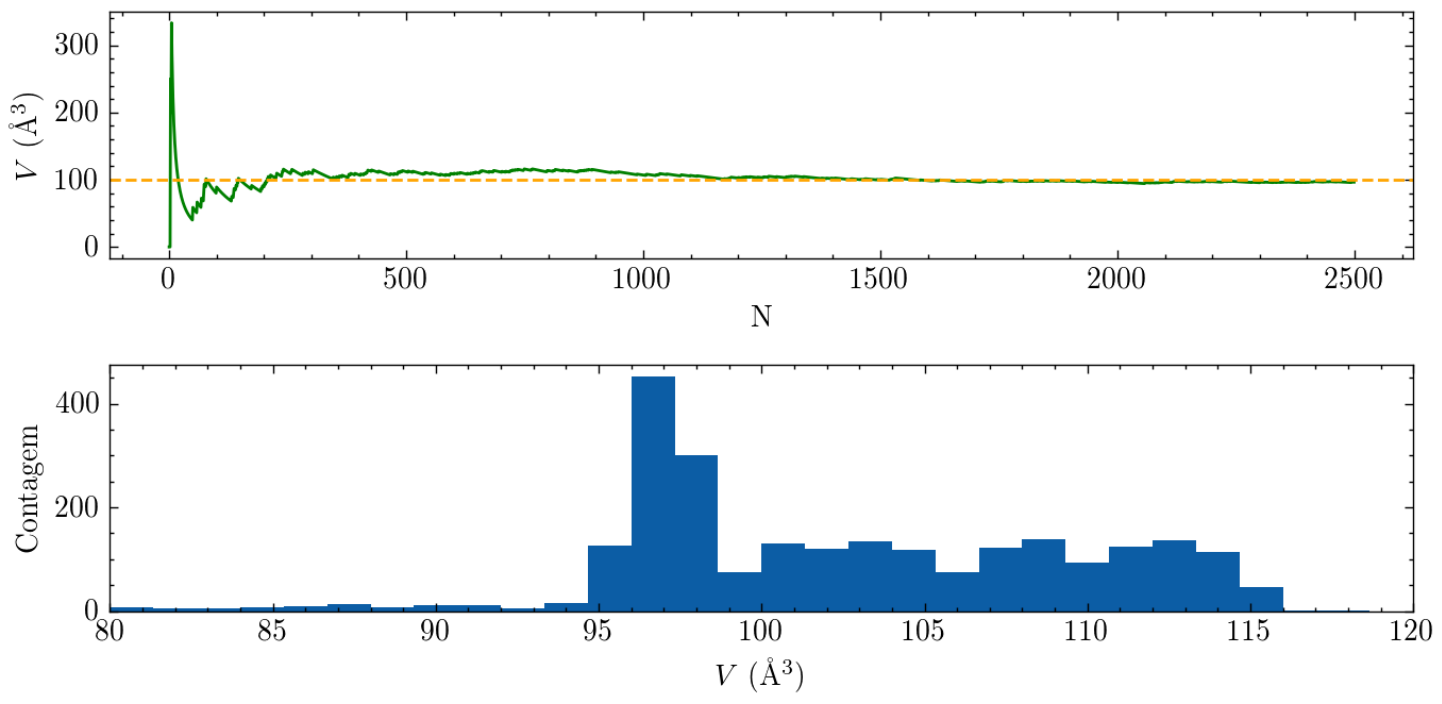

Figura O.2: Evolução do volume para a molécula de tiofeno. O painel superior apresenta o volume $(V)$ em função do número de pontos $(N)$ para os primeiros 2500 pontos. O histograma inferior contabiliza as contribuições desses primeiros 2500 pontos.

Como esperado, após pequenas flutuações iniciais o valor do volume tende a se estabilizar em torno da média, representada pela linha laranja. O mesmo comportamento é observado para os demais compostos, validando a implementação do algoritmo. 